\title{
Limit operators, collective compactness, and the spectral theory of infinite matrices
}

Article

Accepted Version

Chandler-Wilde, S. N. and Lindner, M. (2011) Limit operators, collective compactness, and the spectral theory of infinite matrices. Memoirs of the American Mathematical Society, 210. 989. ISSN 1947-6221 Available at https://centaur.reading.ac.uk/27337/

It is advisable to refer to the publisher's version if you intend to cite from the work. See Guidance on citing.

Published version at: http://www.ams.org/journals/memo/2011-210-989/S0065-9266-2010-00626-4/home.html

Publisher: American Mathematical Society

All outputs in CentAUR are protected by Intellectual Property Rights law, including copyright law. Copyright and IPR is retained by the creators or other copyright holders. Terms and conditions for use of this material are defined in the End User Agreement.

\section{www.reading.ac.uk/centaur}

\section{CentAUR}

Central Archive at the University of Reading

Reading's research outputs online 


\title{
Limit Operators, Collective Compactness, and the Spectral Theory of Infinite Matrices
}

\author{
Simon N. Chandler-Wilde \\ Marko Lindner
}

Author address:

Department of Mathematics, University of Reading, Whiteknights, PO Box 220, Reading RG6 6AX, United Kingdom

E-mail address: S.N.Chandler-Wilde@reading.ac.uk

Fakultät Mathematik, TU Chemnitz, D-09107 Chemnitz, Germany

E-mail address: Marko.Lindner@mathematik.tu-chemnitz.de 

Dedicated to Professor Bernd Silbermann on the occasion of his 67th birthday. 



\section{Contents}

$\begin{array}{ll}\text { Chapter 1. Introduction } & 1\end{array}$

1.1. Overview 1

1.2. A Brief History 5

1.3. Summary and the Main New Results 14

$\begin{array}{lll}\text { Chapter 2. } & \text { The Strict Topology } & 19\end{array}$

\begin{tabular}{lll}
\hline Chapter 3. & Classes of Operators & 26
\end{tabular}

3.1. Compactness and Collective Compactness on $(Y, s) \quad 31$

\begin{tabular}{ll|l}
3.2. & Algebraic Properties & 35
\end{tabular}

\begin{tabular}{lll}
\hline Chapter 4. & Notions of Operator Convergence & 37
\end{tabular}

$\begin{array}{lll}\text { Chapter 5. Key Concepts and Results } & 43\end{array}$

5.1. Invertibility at Infinity and Fredholmness 43

5.2. A Generalised Collectively Compact Operator Theory 44

5.3. Limit Operators 46

5.4. Collective Compactness and the Operator Spectrum 51

$\begin{array}{lll}\text { Chapter } 6 . & \text { Operators on } \ell^{p}\left(\mathbb{Z}^{N}, U\right) & 55\end{array}$

6.1. Periodic and Almost Periodic Operators 56

6.2. Dual Space Arguments 60

6.3. Band-Dominated Operators 64

6.4. Almost Periodic Band-Dominated Operators 73

\begin{tabular}{lll}
\hline 6.5. & The Wiener Algebra & 79
\end{tabular}

\begin{tabular}{|lll}
\hline Chapter 7. & Discrete Schrödinger Operators & 84
\end{tabular}

\begin{tabular}{lll}
\hline Chapter 8. A Class of Integral Operators & 91
\end{tabular}

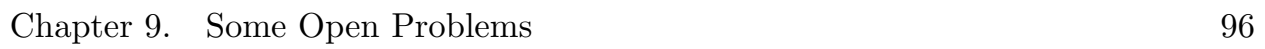

\begin{tabular}{lr}
\hline Bibliography & 98 \\
\hline
\end{tabular}

$\begin{array}{ll}\text { Index } & 102\end{array}$ 


\begin{abstract}
In the first half of this text we explore the interrelationships between the abstract theory of limit operators (see e.g. the recent monographs of Rabinovich, Roch and Silbermann [74 and Lindner [51]) and the concepts and results of the generalised collectively compact operator theory introduced by Chandler-Wilde and Zhang 23. We build up to results obtained by applying this generalised collectively compact operator theory to the set of limit operators of an operator $A$ (its operator spectrum). In the second half of this text we study bounded linear operators on the generalised sequence space $\ell^{p}\left(\mathbb{Z}^{N}, U\right)$, where $p \in[1, \infty]$ and $U$ is some complex Banach space. We make what seems to be a more complete study than hitherto of the connections between Fredholmness, invertibility, invertibility at infinity, and invertibility or injectivity of the set of limit operators, with some emphasis on the case when the operator $A$ is a locally compact perturbation of the identity. Especially, we obtain stronger results than previously known for the subtle limiting cases of $p=1$ and $\infty$. Our tools in this study are the results from the first half of the text and an exploitation of the partial duality between $\ell^{1}$ and $\ell^{\infty}$ and its implications for bounded linear operators which are also continuous with respect to the weaker topology (the strict topology) introduced in the first half of the text. Results in this second half of the text include a new proof that injectivity of all limit operators (the classic Favard condition) implies invertibility for a general class of almost periodic operators, and characterisations of invertibility at infinity and Fredholmness for operators in the so-called Wiener algebra. In two final chapters our results are illustrated by and applied to concrete examples. Firstly, we study the spectra and essential spectra of discrete Schrödinger operators (both selfadjoint and non-self-adjoint), including operators with almost periodic and random potentials. In the final chapter we apply our results to integral operators on $\mathbb{R}^{N}$.
\end{abstract}

2000 Mathematics Subject Classification. Primary 47A53, 47B07; Secondary 46N20, 46E40, 47B37, 47L80.

Key words and phrases. infinite matrices, limit operators, collective compactness, Fredholm operators, spectral theory. 


\section{CHAPTER 1}

\section{Introduction}

\subsection{Overview}

This text develops an abstract theory of limit operators and a generalised collectively compact operator theory which can be used separately or together to obtain information on the location in the complex plane of the spectrum, essential spectrum, and pseudospectrum for large classes of linear operators arising in applications. We have in mind here differential, integral, pseudo-differential, difference, and pseudo-difference operators, in particular operators of all these types on unbounded domains. This text also illustrates this general theory by developing, in a more complete form than hitherto, a theory of the limit operator method in one of its most concrete forms, as it applies to bounded linear operators on spaces of sequences, where each component of the sequence takes values in some Banach space. Finally, we apply this concrete form of the theory to the analysis of lattice Schrödinger operators and to the study of integral operators on $\mathbb{R}^{N}$.

Let us give an idea of the methods and results that we will develop and the problems that they enable us to study. Let $Y=\ell^{p}=\ell^{p}(\mathbb{Z}, \mathbb{C})$, for $1 \leq p \leq \infty$, denote the usual Banach space of complex-valued bilateral sequences $x=(x(m))_{m \in \mathbb{Z}}$ for which the norm $\|x\|$ is finite; here $\|x\|:=\sup _{m}|x(m)|$, in the case $p=\infty$, while $\|x\|:=\left(\sum_{m \in \mathbb{Z}}|x(m)|^{p}\right)^{1 / p}$ for $1 \leq p<\infty$. Let $L(Y)$ denote the space of bounded linear operators on $Y$, and suppose $A \in L(Y)$ is given by the rule

$$
A x(m)=\sum_{n \in \mathbb{Z}} a_{m n} x(n), \quad m \in \mathbb{Z},
$$

for some coefficients $a_{m n} \in \mathbb{C}$ which we think of as elements of an infinite matrix $[A]=\left[a_{m n}\right]_{m, n \in \mathbb{Z}}$ associated with the operator $A$. Of course $A$, given by 1.1 , is only a bounded operator on $Y$ under certain constraints on the entries $a_{m n}$. Simple conditions that are sufficient to guarantee that $A \in L(Y)$, for $1 \leq p \leq \infty$, are to require that the entries are uniformly bounded, i.e.

$$
\sup _{m, n}\left|a_{m n}\right|<\infty
$$

and to require that, for some $w \geq 0, a_{m n}=0$ if $|m-n|>w$. If these conditions hold we say that $[A]$ is a band matrix with band-width $w$ and that $A$ is a band operator. We note that the tri-diagonal case $w=1$, when $A$ is termed a Jacobi operator, is much-studied in the mathematical physics literature (e.g. [89, 47]). This class includes, in particular, the one-dimensional discrete Schrödinger operator for which $a_{m n}=1$ for $|m-n|=1$.

It is well known (see Lemma 6.39 below and the surrounding remarks) that, under these conditions on $[A]$ (that $[A]$ is a band matrix and $[1.2$ holds), the 
spectrum of $A$, i.e. the set of $\lambda \in \mathbb{C}$ for which $\lambda I-A$ is not invertible as a member of the algebra $L(Y)$, is independent of $p$. One of our main results in Section 6.5 implies that also the essential spectrum of $A$ (by which we mean the set of $\lambda$ for which $\lambda I-A$ is not a Fredholm operator ${ }^{1}$ is independent of $p$. Moreover, we prove that the essential spectrum is determined by the behaviour of $A$ at infinity in the following precise sense.

Let $h=(h(j))_{j \in \mathbb{N}} \subset \mathbb{Z}$ be a sequence tending to infinity for which it holds that $a_{m+h(j), n+h(j)}$ approaches a limit $\tilde{a}_{m, n}$ for every $m, n \in \mathbb{Z}$. (The existence of many such sequences is ensured by the theorem of Bolzano-Weierstrass and a diagonal argument.) Then we call the operator $A_{h}$, with matrix $\left[A_{h}\right]=\left[\tilde{a}_{m n}\right]$, a limit operator of the operator $A$. Moreover, following e.g. 74, we call the set of limit operators of $A$ the operator spectrum of $A$, which we denote by $\sigma^{\mathrm{op}}(A)$. In terms of these definitions our results imply that the essential spectrum of $A$ (which is independent of $p \in[1, \infty]$ ) is the union of the spectra of the elements $A_{h}$ of the operator spectrum of $A$ (again, each of these spectra is independent of $p$ ). Moreover, this is also precisely the union of the point spectra (sets of eigenvalues) of the limit operators $A_{h}$ in the case $p=\infty$, in symbols

$$
\operatorname{spec}_{\text {ess }}(A)=\cup_{A_{h} \in \sigma^{\mathrm{op}}(A)}\left\{\lambda: A_{h} x=\lambda x \text { has a bounded solution } x \neq 0\right\} .
$$

This formula and other related results have implications for the spectrum of $A$. In particular, if it happens that $A \in \sigma^{\mathrm{op}}(A)$ (we call $A$ self-similar in that case), then it holds that

(1.4) $\operatorname{spec}(A)=\operatorname{spec}_{\mathrm{ess}}(A)=\cup_{A_{h} \in \sigma^{\mathrm{op}}(A)}\left\{\lambda: A_{h} x=\lambda x\right.$ has a bounded solution $\}$.

In the case $A \notin \sigma^{\mathrm{op}}(A)$ we do not have such a precise characterisation, but if we construct $B \in L(Y)$ such that $A \in \sigma^{\mathrm{op}}(B)$ (see e.g. [51, §3.8.2] for how to do this), then it holds that

(1.5) $\operatorname{spec}(A) \subset \operatorname{spec}_{\text {ess }}(B)=\cup_{B_{h} \in \sigma^{\text {op }}(B)}\left\{\lambda: B_{h} x=\lambda x\right.$ has a bounded solution $\}$.

A main aim of this text is to prove results of the above type which apply in the simple setting just outlined, but also in the more general setting where $Y=\ell^{p}\left(\mathbb{Z}^{N}, U\right)$ is a space of generalised sequences $x=x(m)_{m \in \mathbb{Z}^{N}}$, for some $N \in \mathbb{N}$, taking values in some Banach space $U$. In this general setting the definition (1.1) makes sense if we replace $\mathbb{Z}$ by $\mathbb{Z}^{N}$ and understand each matrix entry $a_{m n}$ as an element of $L(U)$. Such results are the concern of Chapter 6, and are applied to discrete Schrödinger operators and to integral operators on $\mathbb{R}^{N}$ in the final two chapters.

This integral operator application in Chapter 8 will illustrate how operators on $\mathbb{R}^{N}$ can be studied via discretisation. To see how this simple idea works in the case $N=1$, let $G$ denote the isometric isomorphism which sends $f \in L^{p}(\mathbb{R})$ to the sequence $x=(x(m))_{m \in \mathbb{Z}} \in \ell^{p}\left(\mathbb{Z}, L^{p}[0,1]\right)$, where $x(m) \in L^{p}[0,1]$ is given by

$$
(x(m))(t)=f(m+t), \quad m \in \mathbb{Z}, 0<t<1 .
$$

\footnotetext{
${ }^{1}$ Throughout we will say that a bounded linear operator $C$ from Banach space $X$ to Banach space $Y$ is: normally solvable if its range $C(X)$ is closed; semi-Fredholm if, additionally, either $\alpha(C):=\operatorname{dim}(\operatorname{ker} C)$ or $\beta(C):=\operatorname{dim}(Y / C(X))$ are finite; a $\Phi_{+}$operator if it is a semi-Fredholm operator with $\alpha<\infty$, and a $\Phi_{-}$operator if it is semi-Fredholm with $\beta<\infty$; Fredholm if it is semi-Fredholm and both $\alpha$ and $\beta$ are finite. If $C$ is semi-Fredholm then $\alpha(C)-\beta(C)$ is called the index of $C$.
} 
Then the spectral properties of an integral operator $\mathrm{K}$ on $L^{p}(\mathbb{R})$, whose action is given by

$$
\mathrm{K} f(t)=\int_{\mathbb{R}} k(s, t) f(s) d s, \quad t \in \mathbb{R},
$$

for some kernel function $k$, can be studied by considering its discretisation $K:=$ $G K G^{-1}$. In turn $K$ is determined by its matrix $[K]=\left[\kappa_{m n}\right]_{m, n \in \mathbb{Z}}$, with $\kappa_{m n} \in$ $L\left(L^{p}[0,1]\right)$ the integral operator given by

$$
\kappa_{m n} g(t)=\int_{0}^{1} k(m+s, n+t) g(s) d s, \quad 0 \leq t \leq 1 .
$$

Let us also indicate how the results we will develop are relevant to differential operators (and other non-zero order pseudo-differential operators). Consider the first order linear differential operator $L$, which we can think of as an operator from $B C^{1}(\mathbb{R})$ to $B C(\mathbb{R})$, defined by

$$
\mathrm{L} y(t)=y^{\prime}(t)+a(t) y(t), \quad t \in \mathbb{R},
$$

for some $a \in B C(\mathbb{R})$. (Here $B C(\mathbb{R}) \subset L^{\infty}(\mathbb{R})$ denotes the space of bounded continuous functions on $\mathbb{R}$ and $B C^{1}(\mathbb{R}):=\left\{x \in B C(\mathbb{R}): x^{\prime} \in B C(\mathbb{R})\right\}$.) In the case when $a(s) \equiv 1$ it is easy to see that $\mathrm{L}$ is invertible. Specifically, denoting $\mathrm{L}$ by $\mathrm{L}_{1}$ in this case and defining $\mathrm{C}_{1}: B C(\mathbb{R}) \rightarrow B C^{1}(\mathbb{R})$ by

$$
\mathrm{C}_{1} y(t)=\int_{\mathbb{R}} \kappa(s-t) y(s) d s
$$

where

$$
\kappa(s):=\left\{\begin{array}{cc}
e^{s}, & s<0, \\
0, & \text { otherwise, }
\end{array}\right.
$$

it is easy to check by explicit calculation that $L_{1} C_{1}=C_{1} L_{1}=I$ (the identity operator). Thus the study of spectral properties of the differential operator $L$ is reduced, through the identity

$$
\mathrm{L}=\mathrm{L}_{1}+\mathrm{M}_{a-1}=\mathrm{L}_{1}(\mathrm{I}+\mathrm{K})
$$

where $\mathrm{M}_{a-1}$ denotes the operator of multiplication by the function $a-1$, to the study of spectral properties of the integral operator $\mathrm{K}=\mathrm{C}_{1} \mathrm{M}_{a-1}$.

This procedure of reduction of a differential equation to an integral equation applies much more generally; indeed the above example can be viewed as a special case of a general reduction of study of a pseudo-differential operator of non-zero order to one of zero order (see e.g. [74, §4.4.4]). One interesting and simple generalisation is to the case where $\underline{L}$ is a matrix differential operator, a bounded operator from $\left(B C^{1}(\mathbb{R})\right)^{M}$ to $(B C(\mathbb{R}))^{M}$ given by

$$
\underline{\mathrm{L}} x(t)=x^{\prime}(t)+A(t) x(t), \quad t \in \mathbb{R},
$$

where $A$ is an $M \times M$ matrix whose entries are in $B C(\mathbb{R})$. Then, modifying the above argument, the study of $\underline{\underline{L}}$ can be reduced to the study of the matrix integral operator $\underline{\mathrm{K}}=\underline{\mathrm{C}}_{A-I}$. Here $\underline{\mathrm{M}}_{A-I}$ is the operator of multiplication by the matrix $A-I$ ( $I$ the identity matrix) and $\underline{\mathrm{C}}$ is the diagonal matrix whose entries are the (scalar) integral operator $\mathrm{C}_{1}$.

Large parts of the generalisation to the case when the Banach space $U$ is infinitedimensional apply only in the case when $A=I+K$, where $I$ is the identity operator and the entries of $[K]=\left[\kappa_{m n}\right]_{m, n \in \mathbb{Z}}$ are collectively compact. (Where $\mathcal{I}$ is some 
index set, a family $\left\{A_{i}: i \in \mathcal{I}\right\}$ of linear operators on a Banach space $U$ is said to be collectively compact if $\left\{A_{i} x: i \in \mathcal{I}, x \in U,\|x\| \leq 1\right\}$ is relatively compact in $U$.) The first half of this text (Chapters $2 \mid 5$ is devoted to developing an abstract theory of limit operators, in which $Y$ is a general Banach space and in which the role of compactness and collective compactness ideas (in an appropriate weak sense) play a prominent role. Specifically we combine the abstract theory of limit operators as expounded recently in [74, Chapter 1] with the generalised collectively compact operator theory developed in $\mathbf{2 3}$, building up in Chapter 5 to general results in the theory of limit operators whose power we illustrate in the second half of the text, deriving results of the type 1.3 .

Let us give a flavour of the general theory we expound in the first half of the text. To do this it is helpful to first put the example we have introduced above in more abstract notation. In the case $Y=\ell^{p}=\ell^{p}(\mathbb{Z}, \mathbb{C})$, let $V_{k} \in L(Y)$, for $k \in \mathbb{Z}$, denote the translation operator defined by

$$
V_{k} x(m)=x(m-k), \quad m \in \mathbb{Z} .
$$

Then it follows from our definition above that $A_{h}$ is a limit operator of the operator $A$ defined by [1.1) if $\left[V_{-h(j)} A V_{h(j)}\right]$ (the matrix representation of $V_{-h(j)} A V_{h(j)}$ ) converges elementwise to $\left[A_{h}\right]$ as $j \rightarrow \infty$. Let us introduce, moreover, $P_{n} \in L(Y)$ defined by

$$
P_{n} x(m)=\left\{\begin{array}{cc}
x(m), & |m| \leq n \\
0, & \text { otherwise. }
\end{array}\right.
$$

Given sequences $\left(y_{n}\right) \subset Y$ and $\left(B_{n}\right) \subset L(Y)$ and elements $y \in Y$ and $B \in L(Y)$ let us write $y_{n} \stackrel{s}{\rightarrow} y$ and say that $\left(y_{n}\right)$ converges strictly to $y$ if the sequence $\left(y_{n}\right)$ is bounded and

$$
\left\|P_{m}\left(x_{n}-x\right)\right\| \rightarrow 0 \text { as } n \rightarrow \infty,
$$

for every $m$, and write $B_{n} \stackrel{\mathcal{P}}{\rightarrow} B$ if the sequence $\left(B_{n}\right)$ is bounded and

$$
\left\|P_{m}\left(B_{n}-B\right)\right\| \rightarrow 0 \text { and }\left\|\left(B_{n}-B\right) P_{m}\right\| \rightarrow 0 \text { as } n \rightarrow \infty,
$$

for every $m$. Then $A_{h}$ is a limit operator of $A$ if

$$
V_{-h(n)} A V_{h(n)} \stackrel{\mathcal{P}}{\rightarrow} A_{h} .
$$

Defining, moreover, for $b=(b(m))_{m \in Z} \in \ell^{\infty}$, the multiplication operator $M_{b} \in$ $L(Y)$ by

$$
M_{b} x(m)=b(m) x(m), \quad m \in \mathbb{Z},
$$

we note that $A$ is a band operator with band width $w$ if and only if $A$ has a representation in the form

$$
A=\sum_{|k| \leq w} M_{b_{k}} V_{k}
$$

for some $b_{k} \in \ell^{\infty}$. The set $B O(Y)$ of band operators on $Y$ is an algebra. The Banach subalgebra of $L(Y)$ that is the closure of $B O(Y)$ in operator norm will be called the algebra of band-dominated operators, will be denoted by $B D O(Y)$, and will play a main role in the second half of the text, from Chapter 6 onwards.

In the general theory we present in the first five chapters, following [74] and [23, $Y$ becomes an arbitrary Banach space, the specific operators $P_{n}$ are replaced by a a sequence $\mathcal{P}=\left(P_{n}\right)_{n=0}^{\infty}$ of bounded linear operators on $Y$, satisfying constraints 
specified at the beginning of Chapter 2, the specific translation operators $V_{n}$ are replaced by a more general discrete group of isometric isomorphisms, and then the definitions (1.8), 1.9), and (1.10) are retained in essentially the same form. The notion of compactness that proves important is with respect to what we term (adapting the definition of Buck [10]) the strict topology on $Y$, a topology in which $\stackrel{s}{\rightarrow}$ is the sequential convergence. Moreover, when we study operators of the form $A=I+K$ it is not compactness of $K$ with respect to the strict topology that we require (that $K$ maps a neighbourhood of zero to a relatively compact set), but a weaker notion, that $K$ maps bounded sets to relatively compact sets, operators having this property sometimes denoted Montel in the topological vector space literature. (The notions 'compact' and 'Montel' coincide in normed spaces; indeed this is also the case in metrisable topological vector spaces.)

In the remainder of this introductory chapter, building on the above short overview and flavour of the text, we detail a history of the limit operator method and compactness ideas applied in this context, with the aim of putting the current text in the context of extensive previous developments in the study of differential and pseudo-differential equations on unbounded domains; in this history, as we shall see, a prominent role and motivating force has been the development of theories for operators with almost periodic coefficients. In the last section we make a short, but slightly more detailed summary of the contents of the chapters to come.

\subsection{A Brief History}

The work reported in this text has a number of historical roots. One we have already mentioned is the paper by Buck [10] whose strict topology we adapt and use throughout this text. A main thread is the development of limit operator ideas. The historical development of this thread of research, which commences with the study of differential equations with almost periodic solutions, can be traced through the papers of Favard [33, Muhamadiev [57, 58, 59, 60, Lange and Rabinovich 44, 45, 46, culminating in more recent work of Rabinovich, Roch and Silbermann [72, 73, 74. The other main historical thread, which has developed rather independently but overlaps strongly, is the development of collectively compact operator theory and generalisations of this theory, and its use to study well-posedness and stability of approximation methods for integral and other operator equations.

Limit Operators. To our knowledge, the first appearance of limit operator ideas is in a 1927 paper of Favard [33, who studied linear ordinary differential equations with almost periodic coefficients. His paper deals with systems of ODEs on the real line with almost periodic coefficients, taking the form

$$
x^{\prime}(t)+A(t) x(t)=f(t), \quad t \in \mathbb{R},
$$

where the $M \times M$ matrix $A(t)$ has entries that are almost periodic functions of $t$ and the function $f$ is almost periodic. A standard characterisation of almost periodicity is the following. Let $\mathcal{T}(A):=\left\{V_{s} A: s \in \mathbb{R}\right\}$ denote the set of translates of $A$ (here $\left(V_{s} A\right)(t)=A(t-s)$ ). Then the coefficients of $A$ are almost periodic if and only if $\mathcal{T}(A)$ is relatively compact in the norm topology on $B C(\mathbb{R})$. If $A$ is almost periodic, the compact set that is the closure of $\mathcal{T}(A)$ is often denoted $\mathcal{H}(A)$ 
and called the hull of $A$. A main result in 33 is the following: if

$$
x^{\prime}(t)+\tilde{A}(t) x(t)=0, \quad t \in \mathbb{R},
$$

has only the trivial solution in $B C^{1}(\mathbb{R})$, for all $\tilde{A} \in \mathcal{H}(A)$, and 1.13 has a solution in $B C^{1}(\mathbb{R})$, then 1.13 has a solution that is almost periodic. (Since $A \in \mathcal{H}(A)$, this is the unique solution in $B C^{1}(\mathbb{R})$.)

Certain of the ideas and concepts that we use in this text are present already in this first paper, for example the role in this concrete setting of the strict convergence $\stackrel{s}{\rightarrow}$ and of compactness arguments. In particular, conditions analogous to the requirement that (1.14) have no non-trivial bounded solutions for all $A \in \mathcal{H}(A)$ will play a strong role in this text. Conditions of this type are sometimes referred to as Favard conditions (e.g. Shubin [86, 87, Kurbatov [42, 43, Chandler-Wilde \& Lindner [17).

The first appearance of limit operators per se would seem to be in the work of Muhamadiev [57, [58. In [57 Muhamadiev develops Favard's theory as follows. In terms of the differential operator $\underline{\underline{L}}:\left(B C^{1}(\mathbb{R})\right)^{M} \rightarrow(B C(\mathbb{R}))^{M}$ given by 1.6 , equation 1.13 is

$$
\underline{\mathrm{L}} x=f .
$$

Under the same assumptions as Favard (that $A$ is almost periodic and the Favard condition holds) Muhamadiev proves that $\underline{\mathrm{L}}:\left(B C^{1}(\mathbb{R})\right)^{M} \rightarrow(B C(\mathbb{R}))^{M}$ is a bijection. Combining this result with that of Favard, it follows that $\underline{\mathrm{L}}$ is also a bijection from $\left(A P^{1}(\mathbb{R})\right)^{M}$ to $(A P(\mathbb{R}))^{M}$. (Here $A P(\mathbb{R}) \subset B C(\mathbb{R})$ is the set of almost periodic functions and $A P^{1}(\mathbb{R})=A P(\mathbb{R}) \cap B C^{1}(\mathbb{R})$.) New ideas which play an important role in the proof of these results include a method of approximating almost periodic by periodic functions and the fact that, if $A$ is a periodic function, then injectivity of $\underline{L}$ implies invertibility. (These ideas are taken up in the proofs of Theorems 6.7 and 6.38 in Chapter 6)

Muhamadiev also considers in the same paper the more general situation when the entries of $A$ are in the much larger set $B U C(\mathbb{R}) \subset B C(\mathbb{R})$ of bounded uniformly continuous functions. A key property here (which follows from the Arzela-Ascoli theorem and a diagonal argument) is that, if the sequence $\left(t_{n}\right) \subset \mathbb{R}$ tends to infinity, then $A\left(\cdot-t_{n}\right)$ has a subsequence which is convergent to a limit $\tilde{A}$, uniformly on every finite interval. (Cf. the concept of a rich operator introduced in $\$ 5.3$ ) Denoting by $\operatorname{Lim}(A)$ the set of limit functions $\tilde{A}$ obtained in this way, the following theorem is stated: if 1.14 only has the trivial solution in $B C^{1}(\mathbb{R})$ for every $\tilde{A} \in \operatorname{Lim}(A)$ then $\underline{\tilde{L}}:\left(B C^{1}(\mathbb{R})\right)^{M} \rightarrow(B C(\mathbb{R}))^{M}$ is a bijection for every $\tilde{A} \in \operatorname{Lim}(A)$ (here $\underline{\tilde{L}}$ denotes the operator defined by 1.6 with $A$ replaced by $\tilde{A})$.

This is a key result in the development of limit operator theory and it is a shame that [57 does not sketch what must be an interesting proof (we are told only that it 'is complicated'). Denoting by $\mathrm{M}_{A}$ the operator of multiplication by $A$, the set $\left\{\mathrm{M}_{\tilde{A}}: \tilde{A} \in \operatorname{Lim}(A)\right\}$ is a set of limit operators of the operator $\mathrm{M}_{A}$, and so the set $\{\underline{\tilde{L}}: \tilde{A} \in \operatorname{Lim}(A)\}$ is a set of limit operators of the operator $\underline{\mathbf{L}}$. Thus this result takes the form: if each limit operator $\underline{\tilde{L}}$ is injective, specifically $\underline{\tilde{L}} x=0$ has no non-trivial bounded solution, then each limit operator is invertible. A result of this form is a component in the proof of 1.3 and similar results in this text (and see [17]). In the case that $A$ is almost periodic it is an easy exercise to show that 
$\mathcal{H}(A)=\operatorname{Lim}(A)$, i.e. the hull of $A$ coincides with the set of limit functions of $A$ (cf. Theorem 6.10. Thus this second theorem of Muhamadiev includes his result for the case when $A$ is almost periodic.

The first extension of results of this type to multidimensional problems is the study of systems of partial differential equations in $\mathbb{R}^{N}$ in $[\mathbf{5 8}$. Muhamadiev studies differential operators elliptic in the sense of Petrovskii with bounded uniformly Hölder continuous coefficients, specifically those operators $L$ that are what he terms recurrent, by which he means that $\sigma^{\mathrm{op}}(\underline{\mathrm{L}})=\sigma^{\mathrm{op}}(\underline{\tilde{\mathrm{L}}})$, for all $\underline{\tilde{\mathrm{L}}} \in \sigma^{\mathrm{oP}}(\underline{\mathrm{L}})$. Here $\sigma^{\mathrm{oP}}(\underline{\mathrm{L}})$ is an appropriate version of the operator spectrum of $\underline{\mathrm{L}}$. Precisely, where $A_{p}(t)$, for $t \in \mathbb{R}^{N}$ and for multi-indices $p$ with $|p| \leq r$, is the family of coefficients of the operator $\underline{L}$ (here $r$ is the order of the operator), the differential operator of the same form $\underline{\underline{L}}$ with coefficients $\tilde{A}_{p}(t)$ is a member of $\sigma^{\mathrm{op}}(\underline{\underline{L}})$ if there exists a sequence $t_{k} \rightarrow \infty$ such that, for every $p$

$$
A_{p}\left(t-t_{k}\right) \rightarrow \tilde{A}_{p}(t)
$$

uniformly on compact subsets of $\mathbb{R}^{N}$ as $k \rightarrow \infty$.

The main result he states is for the case where $\underline{L}$ is recurrent and is also, roughly speaking, almost periodic with respect to the first $N-1$ variables. His result takes the form that if a Favard condition is satisfied ( $\underline{\underline{L}} x=0$ has no non-trivial bounded solutions for all $\left.\tilde{\underline{L}} \in \sigma^{\mathrm{oP}}(\underline{\mathrm{L}})\right)$ and if supplementary conditions are satisfied which ensure that approximations to $\underline{L}$ with periodic coefficients have index zero as a mapping between appropriate spaces of periodic functions, then $\underline{L}$ is invertible as an operator between appropriate spaces of bounded Hölder continuous functions.

Muhamadiev's results apply in particular in the case when the coefficients of the differential operator are almost periodic (an almost periodic function is recurrent and its set of limit functions is its hull). Shubin, as part of a review of differential (and pseudo-differential) operators with almost periodic solutions [87, gives a detailed account of Muhamadiev's theory, in the almost periodic scalar case (one case where Muhamadiev's supplementary conditions are satisfied), and of results which relate invertibility in spaces of bounded functions to invertibility in $L^{2}\left(\mathbb{R}^{N}\right)$. Specifically, his paper includes a proof, for a scalar elliptic differential operator $\mathrm{L}$ with $C^{\infty}$ almost periodic coefficients, that the following are equivalent: (i) that the Favard condition holds; (ii) that $\mathrm{L}$ is invertible as an operator on $B C^{\infty}\left(\mathbb{R}^{N}\right)$; (iii) that $\mathrm{L}$ is invertible as an operator on $L^{2}\left(\mathbb{R}^{N}\right)$ in an appropriate sense.

In 59 Muhamadiev continues the study of the same class of differential operators $\mathrm{L}$ on $\mathbb{R}^{N}$, elliptic in the sense of Petrovskii, but now, for some of his results, with no constraints on behaviour of coefficients at infinity beyond boundedness, though his main results require also uniform Hölder continuity of all his coefficients. With this constraint (which, inter alia, is a richness requirement in the sense of \$5.3), he studies Fredholmness (or Noethericity) of $\underline{L}$ considered as a bounded operator between appropriate spaces of bounded Hölder continuous functions. It is in this paper that a connection is first made between Fredholmness of an operator and invertibility of its limit operators. The identical Favard condition to that in $[\mathbf{5 8}$ plays a key role. His main results are the following: (i) that $\underline{L}$ is $\Phi_{+}$iff the Favard condition holds; (ii) that if $\underline{L}$ is $\Phi_{-}$then all the limit operators of $\underline{L}$ are surjective; (iii) (his Theorem 2.5 and his remark on p. 899) that $\underline{L}$ is Fredholm iff all the limit operators of $\underline{L}$ are invertible. We note further that his methods of argument in 
the proof of his Theorem 2.1 show moreover that if $\underline{L}$ is Fredholm then the limit operators of $\underline{\underline{L}}$ are not only invertible but the inverses are also uniformly bounded, i.e.

$$
\sup _{\underline{\tilde{L}} \in \sigma^{\text {op }}(\underline{\mathrm{L}})}\left\|\underline{\tilde{L}}^{-1}\right\|<\infty .
$$

Extensions of these results to give criteria for normal solvability and Fredholmness of $\underline{L}$ as an operator on Sobolev spaces are made in $[\mathbf{6 0}]$.

In 59 Muhamadiev also, briefly, introduces what we can term a weak limit operator. Uniform continuity of the coefficients $A_{p}(t)$ is required to ensure that every sequence $t_{k} \rightarrow \infty$ has a subsequence, which we denote again by $t_{k}$, such that the limits (1.15) exist uniformly on compact subsets (cf. the definition of richness in $\$ 5.3$. The set of all limit operators defined by 1.15 where the convergence is uniform on compact sets we have denoted by $\sigma^{\mathrm{op}}(\underline{\underline{\mathrm{L}}) \text {. Muhamadiev notes that it }}$ is enough to require that the coefficients $A_{p}$ be bounded (and measurable) for the same richness property to hold but with convergence uniformly on compact sets replaced ${ }^{2}$ by weak convergence in $L^{2}\left(\mathbb{R}^{N}\right)$. In the case when the coefficients $A_{p}$ are bounded, the set of limit operators defined by 1.15 where the convergence is weak convergence in $L^{2}\left(\mathbb{R}^{N}\right)$ we will term the set of weak limit operators of $\underline{\underline{L}}$. We note that this set coincides with $\sigma^{\mathrm{op}}(\underline{\mathrm{L}})$ in the case when each $A_{p}$ is uniformly continuous. In 60 Muhamadiev gives criteria for Fredholmness of $\underline{L}$ on certain function spaces in terms of invertibility of each of the weak limit operators of $\underline{L}$.

Muhamadiev's work has been a source of inspiration for the decades that followed. For example, similar to his main results in [59] but much more recently, A. and V. Volpert show that, for a rather general class of scalar elliptic partial differential operators $L$ on rather general unbounded domains and also for systems of such, a Favard condition is equivalent to the $\Phi_{+}$property of $L$ on appropriate Hölder [93, 94, 95] or Sobolev [92, 94, 95, spaces.

Lange and Rabinovich [4], inspired by and building on Muhamadiev's paper [59, carry the idea of (semi-)Fredholm studies by means of limit operators over to the setting of operators on the discrete domain $\mathbb{Z}^{N}$. They give sufficient and necessary Fredholm criteria for the class $B D O(Y)$ of band-dominated operators (as defined after 1.12) and studied in more detail below in 8.3 acting on $Y=$ $\ell^{p}\left(\mathbb{Z}^{N}, \mathbb{C}\right)$ spaces. For $1<p<\infty$, they show that such an operator is Fredholm iff all its limit operators are invertible and if their inverses are uniformly bounded. Their proof combines the limit operator arguments of Muhamadiev [59 with ideas of Simonenko and Kozak [39, 84, 85. for the construction of a Fredholm regulariser of $A$ by a clever assembly of local regularisers. Lange and Rabinovich are thereby the first to completely characterise Fredholmness in terms of invertibility of limit operators for the general class of band-dominated operators on $\ell^{p}\left(\mathbb{Z}^{N}, \mathbb{C}\right)$. Before, Simonenko [84, 85. was able to deal with the subclass of those operators whose coefficients (i.e. matrix diagonals) converge along rays at infinity; later Shteinberg 88 was able to relax this requirement to a condition of slow oscillation at infinity. Lange and Rabinovich require nothing but boundedness of the operator coefficients.

\footnotetext{
${ }^{2}$ We note that, since the coefficients $A_{p}$ are bounded so that the sequence $A_{p}\left(\cdot-t_{k}\right)$ is bounded, requiring that the limits 1.15 exist uniformly on compact subsets is equivalent to requiring convergence $\stackrel{s}{\rightarrow}$ in the strict topology, while weak convergence in $L^{2}\left(\mathbb{R}^{N}\right)$ is equivalent to weak* convergence in $L^{\infty}\left(\mathbb{R}^{N}\right)$
} 
The final section of [4] studies (semi-)Fredholmness of operators in the socalled Wiener algebra $\mathcal{W}$ (see our $\$ 6.5$ ) consisting of all operators

$$
A=\sum_{k \in \mathbb{Z}^{N}} M_{b_{k}} V_{k} \quad \text { with } \quad \sum_{k}\left\|b_{k}\right\|_{\infty}<\infty,
$$

where $b_{k} \in \ell^{\infty}\left(\mathbb{Z}^{N}, \mathbb{C}\right)$ for every $k \in \mathbb{Z}^{N}$ are the coefficients (or diagonals) of the operator $A$ and $V_{k}$ and $M_{b_{k}}$ are the shift and multiplication operators defined in 1.7 and 1.11. Operators $A \in \mathcal{W}$ belong to $B D O(Y)$ for all spaces $Y=\ell^{p}\left(\mathbb{Z}^{N}, \mathbb{C}\right)$, $p \in[1, \infty]$. For $p=\infty$, an analogue of the main result of [59] is formulated (in fact, the proof in 44 literally consists of the sentence 'The proofs of Theorems 4.1 and 4.2 repeat the proofs of Theorems 2.1 and 2.2 in $\mathbf{5 9}$, with obvious amendments.'): $A$ is $\Phi_{+}$iff all its limit operators are injective, i.e. Favard's condition holds; if $A$ is $\Phi_{-}$then all its limit operators are surjective. The paper concludes with a first, simplified version of our Theorem 6.40 below, with a somewhat abbreviated proof: that $A \in \mathcal{W}$ is either Fredholm on all spaces $Y=\ell^{p}\left(\mathbb{Z}^{N}, \mathbb{C}\right), p \in[1, \infty]$, or on none of them. Moreover, the uniform boundedness condition on the inverses of its limit operators is redundant.

From here on we mainly follow the discrete branch of the limit operator story since this is the focus of our text, noting that the further generalisation from scalarvalued to vector-valued $\ell^{p}$ spaces $Y=\ell^{p}\left(\mathbb{Z}^{N}, U\right)$ with an arbitrary complex Banach space $U$ enables us to emulate differential, integral and pseudo-differential operators on $L^{p}\left(\mathbb{R}^{N}\right)$ (e.g. [45]) by operators on the discrete space $Y$ with $U=L^{p}\left([0,1]^{N}\right)$ (see e.g. [43, 73, the discussion in the paragraphs after equation 1.5 above, and Chapter 8 below).

In the last 10 years, the limit operators of band-dominated operators on the discrete spaces $Y=\ell^{p}\left(\mathbb{Z}^{N}, U\right)$ with $p \in(1, \infty)$ have been extensively studied by Rabinovich, Roch, Silbermann and a small number of their coauthors. The first work of this troika was $[\mathbf{7 2}$, where the results of $[44$ for $p \in(1, \infty)$ are picked up, this time with full proofs, and are extended, utilised and illuminated in connection with other problems and concepts such as the applicability of the so-called finite section method (a truncation method for the approximate solution of corresponding operator equations) and the idea of two different symbol calculi in the factor algebra of $B D O(Y)$ modulus compact operators. Another important result of $\mathbf{7 2}$ is the observation that the limit operator idea is compatible with the local principle of Allan [2] and Douglas [32 for the study of invertibility in non-commutative Banach algebras. The latter result was used to slightly relax the uniform boundedness condition on the inverses of the limit operators in the general Fredholm criterion [72. Corollary 5] and to completely remove this condition in the case of slowly oscillating coefficients [72, Theorem 9].

In 73 the same authors tackle the case when $U$ is an arbitrary Hilbert space under the additional condition that $p=2$ so that $Y=\ell^{2}\left(\mathbb{Z}^{N}, U\right)$ is a Hilbert space too and the set of band-dominated operators on it is a $\mathrm{C}^{*}$-algebra. In this $\mathrm{C}^{*}$ setting, which makes life slightly easier than the more general case when $B D O(Y)$ is merely a Banach algebra, the serious obstacle of an infinite dimensional space $U$ is overcome. The matrix $[A]$ that corresponds to an operator $A \in B D O(Y)$ now has operator entries $a_{i j} \in L(U)$ which are infinite dimensional operators themselves. This changes the Fredholm theory completely: An operator $A$ with only finitely 
many nonzero entries $a_{i j}$ is in general no longer of finite rank - not even compact. That is why Rabinovich, Roch and Silbermann replace the ideal $K(Y)$ of compact operators by another set, later on denoted by $K(Y, \mathcal{P})$, which is the norm closure of the set of all operators $A$ with finitely many nonzero matrix entries. Also this set is contained in $B D O(Y)$, it is an ideal there and it is shown that if for $A \in B D O(Y)$ there exists a $K(Y, \mathcal{P})$-regulariser $B \in L(Y)$, i.e. $A B-I$ and $B A-I$ are in $K(Y, \mathcal{P})$, then automatically $B \in B D O(Y)$. If $U$ is finite dimensional and $p \in(1, \infty)$, which was the setting of $\mathbf{7 2}$, then $K(Y, \mathcal{P})$ is the same as $K(Y)$ and invertibility modulo $K(Y, \mathcal{P})$, termed invertibility at infinity in $\mathbf{7 3}$, coincides with invertibility modulo $K(Y)$ alias Fredholmness. So one could argue that in $\mathbf{7 2}$ the subject already was invertibility at infinity which, as a coincidence, turned out to be Fredholmness too. In fact, the major milestone in [73] was to understand that the limit operator method studies invertibility at infinity and not Fredholmness, and therefore the new ideal $K(Y, \mathcal{P})$ was the right one to work with. Fortunately, invertibility at infinity and Fredholmness are closely related properties so that knowledge about one of them already says a lot about the other and so the limit operator method can still be used to make statements about Fredholmness - via invertibility at infinity.

Another problem that occurs when passing to an infinite dimensional space $U$ is that the simple Bolzano-Weierstrass argument (coupled with a diagonal construction) previously showing that, for $A \in B D O(Y)$, every sequence $h=(h(k))_{k \in \mathbb{N}} \subset$ $\mathbb{Z}^{N}$ with $|h(k)| \rightarrow \infty$ has a subsequence $g$ such that the matrix of the translates $\left[V_{-g(k)} A V_{g(k)}\right]=\left[a_{i+g(k), j+g(k)}\right]_{i, j \in \mathbb{Z}^{N}}$ converges entry-wise as $k \rightarrow \infty$, is no longer applicable as the matrix diagonals are bounded sequences in the infinite dimensional space $L(U)$ now. So the class of all operators $A \in B D O(Y)$ for which every such sequence $h$ has a subsequence $g$ with this convergence property (the limiting operator being the limit operator $A_{g}$ ) had to be singled out in [73]. Operators of this class were later on termed rich operators.

There is one more technical subtlety when passing to an infinite dimensional space $U$ : The so-called $\mathcal{P}$-convergence $(1.9)$ that is used in 1.10 is equivalent to strong convergence $B_{n} \rightarrow B$ and $B_{n}^{*} \rightarrow B^{*}$ if $p \in(1, \infty)$ and $U$ is finite dimensional; in fact, this is how it was treated in $\mathbf{7 2}$. So this was another difference to [72] although nothing new since $\mathcal{P}$-convergence was de facto introduced for exactly this purpose by Muhamadiev [59] already.

The next two works in this story were the very comprehensive monograph 74 by the troika Rabinovich, Roch and Silbermann, which summarised the state of the art to which it largely contributed itself, and the $\mathrm{PhD}$ thesis $[\mathbf{4 9}$, of the second author of this text. Both grew at roughly the same time and under mutual inspiration and support. In [74], besides many other things that cannot be discussed here, the case $Y=\ell^{p}\left(\mathbb{Z}^{N}, U\right)$ was successfully treated for arbitrary Banach spaces $U$ and $p \in(1, \infty)$. The gaps at $p \in\{1, \infty\}$ are filled in [49. The challenge about $p=\infty$ is that duality, which is a frequent instrument in the arguments of 72, 73, 74, is more problematic since the dual space of $Y$ is no longer one of the $Y$-spaces at hand. Instead one works with the predual and imposes the existence of a preadjoint operator acting on it. Note that some of these ideas have been picked up and are significantly extended and improved in Section 6.2 below. 
Another important thread that should be mentioned here is the determination not only of Fredholmness but also of the Fredholm index by means of limit operators. The key paper in this respect is [71 by Rabinovich, Roch and Roe, where the case $N=1, p=2, U=\mathbb{C}$ has been studied using $C^{*}$-algebra techniques combined with $K$-theory. The idea is to decompose $Y=\ell^{2}(\mathbb{Z}, \mathbb{C})$ into the subspaces $Y_{-}$and $Y_{+}$that correspond to the negative and the non-negative half axis, respectively, thereby splitting the twosided infinite matrix $[A]$ of $A \in B D O(Y)$ into the four onesided infinite submatrices $\left[A_{--}\right],\left[A_{-+}\right],\left[A_{+-}\right]$and $\left[A_{++}\right]$. Since $\left[A_{-+}\right]$ and $\left[A_{+-}\right]$are compact (note that $U$ has finite dimension), these two blocks can be removed without changing Fredholmness or the index. By a similar argument, for every $m \in \mathbb{N}$, the first $m$ rows and columns of both $\left[A_{--}\right]$and $\left[A_{++}\right]$can be removed without losing any information about Fredholmness and the index. So it is not really surprising that also the index of $A$ is exclusively stored in the asymptotic behaviour of the matrix entries of $\left[A_{--}\right]$and $\left[A_{++}\right]$at infinity, i.e. in the limit operators of $A$. Indeed, calling the index of $A_{ \pm \pm}$, understood as an operator on $Y_{ \pm}$, the \pm -index of $A$, respectively, it is shown in 71 that all limit operators of $A$ with respect to sequences tending to $\pm \infty$ have the same \pm- index as $A$ has, respectively. Since the index of $A$ is the sum of its plus- and its minus-index, this gives a formula for the index of $A$ in terms of plus- and minus-indices of two of its limit operators. The index formula of $\mathbf{7 1}$ was later carried over to the case $N=1$, $p \in(1, \infty), U=\mathbb{C}$ in $[\mathbf{7 8}$. (where it was shown that the index of $A$ does not depend on $p$ - see $\mathbf{5 2}$ for the same result in the setting of a more general Banach space $U$ ), re-proved by completely different techniques (using the sequence of the finite sections of $A$ ) in [75] and generalised to the case of an arbitrary Banach space $U$ in case $A=I+K$ with a locally compact operator $K$ (i.e. all entries of $[K]$ are compact operators on $U$ ) in [70].

The most recent extended account of the limit operator method is the monograph $5 \mathbf{5 1}$ by the second author. Besides a unification of techniques and results of 49 and 74 , an exposition of the topic of infinite matrices, in particular banddominated operators, that is accessible for a wide audience and a number of additions and clarifications to the theory, it also contains the first fruits of the work with the other author of this text. For example, it contains a treatment of boundary integral equations on unbounded surfaces (also see [15, 16]), their Fredholmness and finite sections, as well as more complete results on the interplay of Fredholmness and invertibility at infinity and on different aspects of the finite section method.

The above is an account of the main development of limit operator ideas and the theory of limit operators, starting with the work of Favard 33 . However, we have omitted mention of a number of instances where limit-operator-type ideas have been discovered independently. Recent examples are the papers of Davies [27] and Last and Simon [47, which treat specific Schrödinger and Jacobi operators by limitoperator-type arguments. Another fairly substantial body of work, in which limit operator ideas are important, has grown out of the collectively compact operator theory of Anselone and co-authors [3]. It seems appropriate to summarise the main historical developments in this line of research in separate paragraphs below since this body of work work has in common that it is characterised by collective compactness concepts. However, in much of this work limit-operator-type ideas also play a key role. Specifically, limit operator ideas combined with collectively compact operator theory are used already in the 1985 paper of Anselone and Sloan 
[5] to show the stability in $B C[0, \infty)$ of the finite section method for the classical Wiener-Hopf integral equation

$$
y(s)=x(s)+\int_{0}^{\infty} \kappa(s-t) y(t) d t, \quad s \geq 0,
$$

with $\kappa \in L^{1}(\mathbb{R})$. (The finite section method is just the approximation of 1.17 ) by the equation on the finite interval

$$
y(s)=x(s)+\int_{0}^{A} \kappa(s-t) y(t) d t, \quad 0 \leq s \leq A,
$$

and the main issue is to study stability and convergence as $A \rightarrow \infty$.) The methods and results of $\mathbf{5}$ ] are generalised in Chandler-Wilde $[\mathbf{1 3}$ to obtain criteria in $B C(\mathbb{R})$ for both stability of the finite section method and solvability for the equation

$$
y(s)=x(s)+\int_{-\infty}^{\infty} \kappa(s-t) z(t) y(t) d t, \quad s \in \mathbb{R},
$$

in operator form

$$
y=x+K_{z} y,
$$

where $K_{z}$ is the integral operator with kernel $\kappa(s-t) z(t)$, and it is assumed that $\kappa \in L^{1}(\mathbb{R})$ and $z \in L^{\infty}(\mathbb{R})$.

Limit operators do not appear explicitly in $\mathbf{1 3}$, or in generalisations of this work to multidimensional cases [20, 23, to more general classes of kernels 24, to other functions spaces $\left(L^{p}(\mathbb{R}), 1 \leq p \leq \infty\right.$, or weighted spaces) [6, 7], or to general operator equations on Banach spaces [23. Rather, as we discuss in the paragraphs below, the results of these papers provide criteria for unique solvability of 1.18) expressed in terms of injectivity in $B C(\mathbb{R})$ (or equivalently in $L^{\infty}(\mathbb{R})$ ) of the elements of a particular family of operators. The connection to limit operators, explored in Section 5.3 below, is that this family of operators necessarily contains both the operator $I-K_{z}$ and all the weak limit operators of $I-K_{z}$. (Here weak limit operator has the same meaning as in our discussion of the paper $[\mathbf{5 9}$ on page 8 above; we call $K_{\tilde{z}}$ a weak limit operator of $K_{z}$ if, for some unbounded sequence $\left(t_{k}\right) \subset \mathbb{R}$, it holds that $z\left(\cdot-t_{k}\right) \stackrel{w *}{\rightarrow} \tilde{z}$ as $k \rightarrow \infty$, where $\stackrel{w *}{\rightarrow}$ is weak* convergence in $L^{\infty}(\mathbb{R})$.)

Collective Compactness. In the mid 1960's Anselone and co-workers (see [3] and the references therein) introduced the concept of collectively compact operators. A family $\mathcal{K}$ of linear operators on a Banach space $Y$ is called collectively compact if, for any sequences $\left(K_{m}\right) \subset \mathcal{K}$ and $\left(x_{m}\right) \subset Y$ with $\left\|x_{m}\right\| \leq 1$, there is always a subsequence of $\left(K_{m} x_{m}\right)$ that converges in the norm of $Y$. It is immediate that every collectively compact family $\mathcal{K}$ is bounded and that all of its members are compact operators.

There are some important features of collectively compact sets of operators. First, recall that if $K$ is a compact operator on $Y$ and a sequence $A_{m}$ of operators on $Y$ converges strongly (i.e. pointwise) to 0 , then $A_{m} K$ converges to 0 in the operator norm on $Y$. But under the same assumption, even $A_{m} K_{m}$ converges to 0 in the norm for any sequence $\left(K_{m}\right) \subset \mathcal{K}$ provided $\mathcal{K}$ is collectively compact. This fact was probably the motivation for the introduction of this notion. It was used by 
Anselone for the convergence analysis of approximation methods like the Nyström method for second kind integral equations.

Another important feature [3. Theorem 1.6] is that if $\left\{K_{m}\right\}_{m=1}^{\infty}$ is collectively compact and strongly convergent to $K$, then also $K$ is compact, and the following holds:

$$
\begin{aligned}
& I-K \text { is invertible } \quad \Longleftrightarrow \\
& I-K_{m} \text { is invertible for large } m \text {, say } m>m_{0} \text {, and } \sup _{m>m_{0}}\left\|\left(I-K_{m}\right)^{-1}\right\|<\infty
\end{aligned}
$$

Since $K$ and $K_{m}$ are compact, the above is equivalent to the following statement

$$
I-K \text { is injective } \Longleftrightarrow \quad \exists m_{0}: \inf _{m>m_{0}} \nu\left(I-K_{m}\right)>0
$$

where $\nu(A):=\inf \{\|A x\|:\|x\|=1\}$ is the so-called lower norm of an operator $A$.

There are many important examples where $K$ is not compact in the norm topology on $Y$ but does have compactness properties in a weaker topology. To be precise, $K$, while not compact (mapping a neighbourhood of zero to a relatively compact set) has the property that, in the weaker topology, it maps bounded sets to sets that are relatively compact (such operators are sometimes termed Montel) ${ }^{3}$. In particular, this is generically the case when $K$ is an integral operator on an unbounded domain with a continuous or weakly singular kernel; these properties of the kernel make $K$ a 'smoothing' operator, so that $K$ has local compactness properties, but $K$ fails to be compact because the domain is not compact. Anselone and Sloan [5] were the first to extend the arguments of collectively compact operator theory to tackle a case of this type, namely to study the finite section method for classical Wiener-Hopf operators on the half-axis. As mentioned already above, the arguments introduced were developed into a methodology for establishing existence from uniqueness for classes of second kind integral equations on unbounded domains and for analyzing the convergence and stability of approximation methods in a series of papers by the first author and collaborators $[13,63,20,24,56,18,23,6,17$. A particular motivation for this was the analysis of integral equation methods for problems of scattering of acoustic, elastic and electromagnetic waves by unbounded surfaces 14, 21, 96, 19, 22, 56, 18, 97, 61, 7, 16. Other applications included the study of multidimensional Wiener-Hopf operators and, related to the Schrödinger operator, a study of Lippmann-Schwinger integral equations [20]. Related developments of the ideas of Anselone and Sloan $\mathbf{5}$ to the analysis of nonlinear integral equations on unbounded domains are described in [1, 4, 62.

In 23 the first author and Zhang put these ideas into the setting of an abstract Banach space $Y$, in which a key role is played by the notion of a generalised collectively compact family $\mathcal{K}$. Now the sequence $\left(K_{m} x_{m}\right)$ has a subsequence that converges in a topology that is weaker than the norm topology on $Y$, whenever $\left(K_{m}\right) \subset \mathcal{K}$ and $\left(x_{m}\right) \subset Y$ with $\left\|x_{m}\right\| \leq 1$. This notion no longer requires the elements of $\mathcal{K}$ to be compact operators and therefore covers a lot more operators originating from applications. But still, the following similar result to 1.19 was established in $2 \mathbf{2 3}$. If $\mathcal{K}$ is generalised collectively compact (or uniformly Montel as we shall term the same property in Definition 3.22 in this text) and some important

\footnotetext{
${ }^{3}$ Already in the 1970's DePree and co-authors [29, 30, 31 studied collectively compact operator theory in a topological vector space setting, but they retained compactness of $K$ rather than studying the weaker case where $K$ is Montel.
} 
additional assumptions hold (see the end of our discussion of limit operators above and Theorem 5.9 below), then

$$
I-K \text { is injective for all } K \in \mathcal{K} \quad \Longleftrightarrow \quad \inf _{K \in \mathcal{K}} \nu(I-K)>0 \text {. }
$$

If the family $\mathcal{K}$ satisfies rather strong additional constraints (see Theorem 5.9 below for details), then also invertibility of $I-K$ for every $K \in \mathcal{K}$ follows from injectivity for all $K \in \mathcal{K}$.

To give a concrete flavour of these results (this was the first concrete application of these ideas made to boundary integral equations in wave scattering [13, 14), one case where they apply is to the integral equation $(1.18)$, with the family $\mathcal{K}$ defined by

$$
\mathcal{K}:=\left\{K_{z}: z \in L^{\infty}(\mathbb{R}) \text { and } z(s) \in Q, \text { for almost all } s \in \mathbb{R}\right\},
$$

for some $Q \subset \mathbb{C}$ which is compact and convex. That is, existence and uniform boundedness of $\left(I-K_{z}\right)^{-1}$ (as an operator on $B C(\mathbb{R})$ ) for all $K_{z} \in \mathcal{K}$, can be shown to follow from injectivity of $I-K_{z}$ for all $K_{z} \in \mathcal{K}$ (see [13, 14] or [23 for details).

Generalised Collective Compactness and Limit Operators: A Main Aim of this Memoir. We have briefly indicated above some connections between the two bodies of work that we have described under the headings 'Limit Operators' and 'Collective Compactness'. It was a main aim for us in writing this text to explore these connections in a methodical way, in particular to explore the possibilities for applying the generalised collectively compact operator theory [23 in a limit operator context, and for combining collective compactness and limit operator ideas. It turns out that these ideas have a very fruitful interplay (other recent examples of this interplay in addition to this text are $\mathbf{7 0}$ and 17, the latter paper making use of some of the results we will present below). We finish this introduction by summarising the main new results of the text and of this interplay of ideas.

\subsection{Summary and the Main New Results}

In this final section of the introduction, having given an overview of the ideas of the text and their historical development in Sections 1.1 and 1.2, we briefly summarise, chapter by chapter, the main contents and results we obtain.

The text falls into two connected parts. The first part, chapters 2-5, is concerned with extensions to the general abstract theory of limit operators, as expounded in Chapter 1 of $\mathbf{7 4}$, with exploring the connections with the abstract generalised collectively compact operator theory of [23, and with making applications of this theory in the limit operator context.

The short initial Chapter 2 introduces, following $\mathbf{7 4}$, the idea of a sequence $\left(P_{n}\right)$ of bounded linear operators on a Banach space $Y$ that form an approximate

\footnotetext{
${ }^{4}$ It was results in 49 , in particular where it is pointed out for the first time that the operator spectrum $\sigma^{\mathrm{op}}(A)$ of a rich operator is always relatively sequentially compact with respect to $\mathcal{P}$ convergence - one of the additional assumptions required for Theorem 5.9 below, taken from [23] - which prompted the authors to start to investigate this symbiosis, in discussions after the first author examined the thesis of the second!
} 
identity on $Y$, satisfying conditions (i) and (ii) at the beginning of the chapter. In the theory of limit operators developed in $\mathbf{7 4}$ the following notion of sequential convergence plays a crucial role: we write that $x_{n} \stackrel{s}{\rightarrow} x$ if $\left(x_{n}\right)$ is a bounded sequence and $P_{m} x_{n} \rightarrow P_{m} x$ as $n \rightarrow \infty$ for every $m$. In this text, as we have noted already, we call $\stackrel{s}{\rightarrow}$ strict convergence, by analogy with the strict topology of [10]. Chapter 2 is concerned with study of a topology, which we term the strict topology, in which $\stackrel{s}{\rightarrow}$ is the sequential convergence. We recall properties of this topology from 23. (which derive in large part from similar results in [10]) and show further properties, for example characterising the compact and sequentially compact sets in the strict topology, characterising when the strict and norm topologies coincide, and introducing many examples that we build on later.

In Chapter 3 we study a number of subspaces of $L(Y)$, the space of bounded linear operators on the Banach space $Y$, namely those subspaces that play an important role in the abstract theory of limit operators [74] and in the generalised collectively compact operator theory of [23, and so play an important role in the rest of the text. These subspaces include the classes $L(Y, \mathcal{P})$ and $K(Y, \mathcal{P})$ central to the theory of limit operators $[\mathbf{7 4}]^{5}$, the class $S(Y)$ of operators that are sequentially continuous on $(Y, s)$ ( $Y$ equipped with the strict topology), and the class $S N(Y)$ of operators that are sequentially continuous from $(Y, s)$ to $(Y,\|\cdot\|)(Y$ equipped with the norm topology). We also study the usual class $K(Y)$ of compact operators on $(Y,\|\cdot\|)$, the class $K S(Y)$ of compact operators on $(Y, s)$, and the class $M(Y)$ of Montel operators on $(Y, s)$ (that map bounded sets to relatively compact sets), this latter class playing a particularly key role in the later text. Our concern is to derive explicit characterisations of these sets and to explore the relationships between them. For example we see in this chapter that $S(Y)$ coincides with the set of continuous linear operators on $(Y, s)$, that $S N(Y)$ is the set of continuous linear operators from $(Y, s)$ to $(Y,\|\cdot\|)$, and that $S(Y)$ and $S N(Y)$ are, roughly speaking, "one-sided" versions of $L(Y, \mathcal{P})$ and $K(Y, \mathcal{P})$ (see Lemmas 3.3 and 3.10 . Corollary 3.5 and (3.5)). An easy but informative result, which makes clear that being Montel is a much weaker property than being compact on $(Y, s)$, is Corollary 3.24 which has application for example in Chapter 7, that $M(Y)=L(Y)$ if $(Y, s)$ is sequentially complete and each $P_{n} \in K(Y)$. In Section 3.2 we study algebraic properties, for example showing that all of $S(Y), S N(Y), M(Y)$, and $K S(Y)$ are Banach subalgebras of $L(Y)$, and that $S N(Y), K S(Y)$, and $M(Y) \cap S(Y)$ are all ideals of $S(Y)$.

In the short Chapter 4 we introduce and contrast various notions of convergence of sequences of operators in $L(Y)$, with an emphasis on those used in the abstract theory of limit operators $[\mathbf{7 4}$ and in the generalised collectively compact operator theory of 23. Specifically, our main emphasis is on the $\stackrel{\mathcal{P}}{\rightarrow}$ convergence of Definition 4.1 (and see [80, 74], though the $\stackrel{\mathcal{P}}{\rightarrow}$ convergence in [74] is restricted to operators in $L(Y, \mathcal{P}))$, and on the weaker notions of convergence $(\stackrel{s}{\rightarrow}$ and $\stackrel{S}{\rightarrow})$ important in 23. These three notions of convergence will play a strong role in subsequent chapters, but we also compare these notions of convergence to ordinary norm $(\rightrightarrows)$ and strong $(\rightarrow)$ convergence in $L(Y)$. Informative characterisations we derive are

\footnotetext{
${ }^{5}$ Indeed, in 74 the notion of a limit operator of an operator is defined only for operators in $L(Y, \mathcal{P})$. We will not be this restrictive but extend this notion and other definitions as far as possible to the whole class $L(Y)$.
} 
that a sequence $\left(A_{n}\right) \subset L(Y)$ satisfies $A_{n} \stackrel{\mathcal{P}}{\rightarrow} 0$ if and only if $\left(A_{n}\right)$ is bounded and both $P_{m} A_{n} \rightrightarrows 0$ and $A_{n} P_{m} \rightrightarrows 0$ as $n \rightarrow \infty$, for every $m$, while $A_{n} \stackrel{S}{\rightarrow} 0$ if and only if $\left(A_{n}\right)$ is bounded and $P_{m} A_{n} x \stackrel{s}{\rightarrow} 0$ as $n \rightarrow \infty$ for every $m$ and every $x \in Y$. Thus $A_{n} \rightrightarrows 0 \Rightarrow A_{n} \stackrel{\mathcal{P}}{\rightarrow} 0 \Rightarrow A_{n} \stackrel{S}{\rightarrow} 0 \Leftarrow A_{n} \rightarrow 0$ (cf. Corollary 4.14 .

Chapter 5 introduces the main abstract concepts and results of the text. In Section 5.1 we introduce the concept of invertibility at infinity (Definition 5.1 of an operator $A \in L(Y)$ and show that if $A \in S(Y)$ is a Montel perturbation of an invertible operator in $S(Y)$, then $A$ invertible at infinity implies that $A$ is Fredholm (Theorem 5.4). In Section 5.2 we present a specialisation of some main results of the generalised collectively compact operator theory on a Banach space $Y$ of 23, to the case where the family of seminorms on $Y$ required in the theory of 23 is given by (2.4). In Section 5.3 we make an abstract definition of the limit operators and the operator spectrum, $\sigma^{\mathrm{op}}(A)$ (the set of limit operators), of an arbitrary operator $A \in L(Y)$. We also list the main properties of the operator (including Fredholmness, invertibility at infinity, and injectivity and invertibility of the members of the operator spectrum) that we seek to make connections between in Chapters 5 and 6. New results in this section include: a refinement of $\mathbf{7 4}$, Proposition 1.2.9], that, in the so-called perfect case (when both $P_{n}$ and its adjoint converge strongly to the identity as $n \rightarrow \infty)$, Fredholmness implies invertibility of all the limit operators of $A$ for every $A \in L(Y)$ (not just for $A \in L(Y, \mathcal{P})$ ); the observation that, moreover, Fredholmness is equivalent to invertibility when $A$ is self-similar $\left(A \in \sigma^{\mathrm{op}}(A)\right)$; and a strong existence result for self-similar operators, that every rich operator ('richness' of $A$, defined in Section 5.3 and characterised in Theorem 5.12, guarantees the existence of limit operators) has a self-similar limit operator. Section 5.4 contains what are probably the main new results of this text related to limit operators at an abstract level (Theorems 5.16 and 5.20), obtained by applying the generalised collectively compact operator theory of Section 5.2 . These results, roughly speaking, relate invertibility of an operator $A=I+K$, in the case when $K$ is Montel on $(Y, s)$ (and $\sigma^{\mathrm{op}}(K)$ is uniformly Montel in the sense of Definition 3.22), and stability of an approximating sequence $A_{n}=I+K_{n}$ to $A$, to injectivity of the elements of $\sigma^{\mathrm{op}}(A)$ or of the members of a slightly larger set. One main theme of the rest of the text is demonstrating the applicability of these results, and in fact they are central to the proofs of Theorems 6.3, 6.31 and 6.37. and are important components of the proofs of Corollaries 6.30, 6.32, 6.43, 6.45. 6.46 and 6.48

The largest chapter of this text is Chapter 6, where we apply results of Chapter 5 to the concrete spaces $Y=Y^{p}:=\ell^{p}\left(\mathbb{Z}^{N}, U\right)$ and $Y=Y^{0}:=c_{0}\left(\mathbb{Z}^{N}, U\right)$ with $N \in \mathbb{N}$, $p \in[1, \infty]$ and $U$ a complex Banach space. This is still a fairly general situation, as illustrated e.g. in Chapters 7 and 8 , but it is concrete enough to allow much more precise statements on Fredholmness and invertibility at infinity of operators on $Y$ than was possible in the very general setting of Chapter 5 . We start with a short section on the Banach algebra of almost periodic aka norm-rich operators on $Y$ and their operator spectrum. These results are picked up and improved in a later section on band-dominated and norm-rich operators with the main result that $A=I+K$, with $K$ band-dominated, norm-rich and $\sigma^{\mathrm{op}}(K)$ uniformly Montel, is invertible on $Y^{\infty}$ iff all its limit operators are injective on $Y^{\infty}$, i.e. Favard's condition holds. In Section 6.2 we study interrelations of (semi-) Fredholmness and index of an 
operator $A$ on $Y^{\infty}$ and its restriction $A_{0}$ to $Y^{0}$. The main techniques here are to isometrically embed $Y^{\infty}$ into $\left(Y^{0}\right)^{* *}$ and $Y^{1}\left(U^{*}\right):=\ell^{1}\left(\mathbb{Z}^{N}, U^{*}\right)$ into $\left(Y^{\infty}\right)^{*}$ and to show that, in terms of these embeddings, every $A \in S(Y)$ is the restriction of $A_{0}^{* *}$ to $Y^{\infty}$ and $A_{0}^{*}$ is the restriction of $A^{*}$ to $Y^{1}\left(U^{*}\right)$. Further connections are established in the case that $U$ has a predual $U^{\triangleleft}$ and $A$ is the adjoint of an operator $A^{\triangleleft}$ on $U^{\triangleleft}$. In the following section we introduce $B D O(Y)$, the set of all banddominated operators on $Y$, which is the norm closure in $L(Y)$ of the set of all band operators or, equivalently, the smallest Banach subalgebra of $L(Y)$ that contains all shift operators (1.7) and all multiplication operators 1.11 with an operatorvalued multiplier $b \in \ell^{\infty}\left(\mathbb{Z}^{N}, L(U)\right)$. Using results from Chapters 3,5 and Section 6.2 we then derive criteria for and connections between invertibility at infinity and Fredholmness of $A \in B D O\left(Y^{p}\right)$ that are new or more complete than before if $p \in\{0,1, \infty\}$. The final section of Chapter 6 studies operators in the Wiener algebra $\mathcal{W}$ that we mentioned in (1.16) already. The attraction for studying this subset of $B D O(Y)$ in more depth is that operators in $\mathcal{W}$ act boundedly on every space $Y^{p}$ with $p \in\{0\} \cup[1, \infty]$ and neither their invertibility nor invertibility at infinity, Fredholmness or the Fredholm index depend on the choice of $p$. It results that, while, for general operators $A \in B D O\left(Y^{p}\right)$ in the previous section, the collective compactness arguments from Chapter 5 mainly contribute to the theory for $p=\infty$ (and, via duality, to $p=0$ and $p=1$ ), now for operators $A \in \mathcal{W}$, the same results hold for $p \in(1, \infty)$. For example, one gets that, for $N=1$ and $K \in \mathcal{W}$ rich with $\sigma^{\mathrm{op}}(K)$ uniformly Montel, the operator $A=I+K$ is Fredholm on any of the spaces $Y^{p}$ iff all of its limit operators are injective on $Y^{\infty}$, i.e. Favard's condition holds.

In Chapters 7 and 8 we illustrate our main results for two operator classes of major interest in applications. In Chapter 7 we discuss discrete Schrödinger operators (both self-adjoint and non-self-adjoint), making links to other recent work (e.g Davies [27] and Last \& Simon [47]). In particular, we derive what appear to be new characterisations of the spectrum and essential spectrum (Theorem 7.1 and (7.13), in terms of the point spectrum in $\ell^{\infty}$ of the discrete operator and its limit operators, and apply these results to Schrödinger operators with potentials that are almost-periodic, perturbations of almost-periodic, pseudo-ergodic in the sense of Davies [27, and random, in this latter case reproducing results of Trefethen, Contedini \& Embree 90. In Chapter 8 we demonstrate how the results of Chapter 6 can be applied to continuous operators, deducing criteria for Fredholmness and invertibility for members of a large Banach algebra of integral operators on $\mathbb{R}^{N}$.

We conclude this text by a final chapter that contains a small list of open problems that we consider highly interesting for the further development of this field. 
Acknowledgements. We would like to dedicate this text to Professor Bernd Silbermann, who has been, and still is, an icon and a very influential figure for both of us. When we started this text he was still 62 . But it soon turned out to be a rather long term project, and our plans to dedicate it to his 65th birthday were shattered when we couldn't stop ourselves from continuing to work and write on it. So finally, being an extraordinary man, he is getting the extraordinary honour of a dedication to his $\mathbf{6 7 t h}$ birthday. Happy birthday, Bernd!

We would also like to acknowledge useful discussions of parts of this work with colleagues including Professor Les Bunce (Reading), Professor Brian Davies (King's College, London), Professor Peter Kuchment (Texas A\&M University), Professor Steffen Roch (Darmstadt), and of course Professor Bernd Silbermann (Chemnitz). Finally, we gratefully acknowledge the financial support of a Leverhulme Fellowship and a visiting Fellowship of the Isaac Newton Institute for the first author and of a Marie Curie Fellowship (MEIF-CT-2005-009758) and the Research Grant PERG02GA-2007-224761 of the European Commission for the second author.

Reading and Chemnitz

6 April 2008
Simon N. Chandler-Wilde

Marko Lindner 


\section{CHAPTER 2}

\section{The Strict Topology}

As usual, by $\mathbb{N}, \mathbb{Z}, \mathbb{R}$ and $\mathbb{C}$ we refer to the sets of all natural, integer, real and complex numbers, respectively. We also put $\mathbb{N}_{0}:=\{0,1,2, \ldots\}$ and $\mathbb{R}_{+}:=[0, \infty)$.

Throughout, $Y$ will denote a real or complex Banach space, with norm denoted by $\|\cdot\|$, and $L(Y)$ refers to the set of bounded linear operators on $Y$. $\mathcal{P}=\left(P_{n}\right)_{n=0}^{\infty}$ will denote a sequence of operators in $L(Y)$ with the properties that:

(i) $\sup _{n}\left\|P_{n} x\right\|=\|x\|, x \in Y$;

(ii) for every $m \in \mathbb{N}_{0}$ there exists $N(m) \geq m$ such that

$$
P_{n} P_{m}=P_{m}=P_{m} P_{n}, \quad n \geq N(m) .
$$

Throughout we will write $n \gg m$ or $m \ll n$ if $P_{n} P_{k}=P_{k}=P_{k} P_{n}$ for all $k \leq m$. Note that $l \ll m$ and $m \ll n$ imply $l \ll n$ since $P_{n} P_{k}=P_{n} P_{m} P_{k}=P_{m} P_{k}=P_{k}=$ $P_{k} P_{m}=P_{k} P_{m} P_{n}=P_{k} P_{n}$ for all $k \leq l$.

In $[74$ a bounded sequence $\mathcal{P}$ satisfying (ii) is called an increasing approximate projection and an increasing approximate projection satisfying (i) (or (i) with the '=' replaced by a ' $\geq$ ') is called an approximate identity. Thus $\mathcal{P}$ is an approximate identity in the terminology of [74].

It is easy to see that (i) implies that $\left\|P_{n}\right\| \leq 1$ for all $n$. Moreover, if (i) and (ii) hold, then $\left\|P_{n}\right\|=1$ for all sufficiently large $n$ and, for all $x \in Y$, the limit $\lim _{n \rightarrow \infty}\left\|P_{n} x\right\|$ exists and

$$
\lim _{n \rightarrow \infty}\left\|P_{n} x\right\|=\|x\|, \quad x \in Y .
$$

For all $n \in \mathbb{N}_{0}$ let $Q_{n}:=I-P_{n}$ and note that (ii) implies that, for all $m \in \mathbb{N}_{0}$,

$$
Q_{n} Q_{m}=Q_{n}=Q_{m} Q_{n}, \quad n \geq N(m),
$$

and (i) that $\left\|Q_{n}\right\| \leq 2$ for all $n \in \mathbb{N}_{0}$.

REMARK 2.1 There are two possibilities inherent in the assumptions (i) and (ii): either $P_{n}=I$ for some $n$ or $P_{n} \neq I$ for all $n$. In the first case (ii) implies that $P_{n}=I$ for all sufficiently large $n$. In the second case $Q_{n} \neq 0$ for all $n$ and 2.3 implies that $\left\|Q_{n}\right\| \geq 1$, so that $1 \leq\left\|Q_{n}\right\| \leq 2$ for all $n$. Therefore, unless $P_{n}=I$ for all sufficiently large $n$, it does not hold that $\left\|P_{n}-I\right\| \rightarrow 0$ as $n \rightarrow \infty$. It may happen that $P_{n}$ converges strongly to $I$; this is the case in Example 2.6 below and in Example 2.4. for $1 \leq p<\infty$, but not in Examples 2.2, 2.3, 2.5 or Example 2.4 for $p=\infty$.

EXAmPle 2.2 Let $Y=\ell^{\infty}$, the Banach space of bounded real- or complexvalued sequences $x=(x(m))_{m \in \mathbb{Z}}$, with norm $\|x\|=\sup _{m}|x(m)|$. Define, for $x \in Y$, 
$n \in \mathbb{N}_{0}$,

$$
P_{n} x(m)= \begin{cases}x(m), & |m| \leq n, \\ 0, & |m|>n .\end{cases}
$$

Then $\mathcal{P}=\left(P_{n}\right)$ satisfies (i) and (ii) with $N(m)=m$, so that $P_{n}$ is a projection operator for each $n$. In this case $\left\|Q_{n}\right\|=1$ for all $n$.

Example 2.3 Let $Y=\ell^{\infty}$ as in Example 2.2. Define, for $n \in \mathbb{N}_{0}, P_{n} \in L(Y)$ by $P_{n} x(m)=x(m)$, for $|m| \leq\left(3^{n}-1\right) / 2$, and by the requirement that $P_{n} x\left(m+3^{n}\right)=$ $P_{n} x(m)$, for $m \in \mathbb{Z}$. Then $\mathcal{P}=\left(P_{n}\right)$ satisfies (i) and (ii) with $N(m)=m$, so that $P_{n}$ is a projection operator for each $n$. In this case $\left\|Q_{n}\right\|=2$ for all $n$.

EXAMPLE 2.4 Let $Y=L^{p}\left(\mathbb{R}^{N}\right)$, the Banach space of those Lebesgue measurable real- or complex-valued functions $x$ on $\mathbb{R}^{N}$, for which the norm $\|x\|_{p}$ is finite, where $\|x\|_{p}:=\left(\int_{\mathbb{R}^{N}}|x(s)|^{p} d s\right)^{1 / p}$, for $1 \leq p<\infty$, and $\|x\|_{\infty}:=\operatorname{ess}_{\sup } \operatorname{se}_{\mathbb{R}^{N}}|x(s)|$. Define, for $x \in Y, n \in \mathbb{N}_{0}$,

$$
P_{n} x(s)= \begin{cases}x(s), & |s| \leq n, \\ 0, & |s|>n .\end{cases}
$$

Then $\mathcal{P}=\left(P_{n}\right)$ satisfies (i) and (ii) with $N(m)=m$, so that $P_{n}$ is a projection operator for each $n$. In this case $\left\|Q_{n}\right\|=1$ for all $n$.

EXAmple 2.5 Let $Y=B C\left(\mathbb{R}^{N}\right)$, the Banach space of bounded continuous real- or complex-valued functions on $\mathbb{R}^{N}$, with norm $\|x\|=\sup _{s \in \mathbb{R}^{N}}|x(s)|$. Choose $\chi \in B C(\mathbb{R})$ with $\|\chi\|=1$ and $\chi(t)=0, t \leq 0,=1, t \geq 1$. Define, for $n \in \mathbb{N}_{0}$ and $x \in Y$,

$$
P_{n} x(s)=\chi(n+1-|s|) x(s), \quad s \in \mathbb{R}^{N} .
$$

Then $\mathcal{P}=\left(P_{n}\right)$ satisfies (i) and (ii) with $N(m)=m+1$. In this case $\left\|Q_{n}\right\|=\|1-\chi\|$.

Example 2.6 Let $Y=C[0,1]$ with $\|x\|=\sup _{0 \leq s \leq 1}|x(s)|$ and let $P_{n} x$ denote the piecewise linear function which interpolates $x$ at $j / 2^{n}, j=0,1, \ldots, 2^{n}$. Then $\mathcal{P}=\left(P_{n}\right)$ satisfies (i) and (ii) with $N(m)=m$.

Note that in Examples 2.2 and $2.6 P_{n}$ has finite-dimensional range for each $n$, so that $P_{n} \in K(Y)$, the set of compact linear operators on $Y$. (The significance of this is discussed in Remark 2.16 below.)

Throughout, for $\left(x_{n}\right) \subset Y, x \in Y$, we will write $x_{n} \rightarrow x($ as $n \rightarrow \infty)$ if $\left(x_{n}\right)$ converges to $x$ in the norm topology, i.e. $\left\|x_{n}-x\right\| \rightarrow 0$ as $n \rightarrow \infty$. We will also be concerned with convergence in weaker topologies, defined in terms of semi-norms on $Y$ that are related to the sequence $\mathcal{P}$. For $n \in \mathbb{N}_{0}$ define $|\cdot|_{n}: Y \rightarrow \mathbb{R}_{+}$by

$$
|x|_{n}:=\max _{0 \leq m \leq n}\left\|P_{m} x\right\|, \quad x \in Y .
$$

It is easy to check that $|\cdot|_{n}$ is a semi-norm on $Y$, and (i) and (ii) imply that, for $m<n, x \in Y$,

$$
|x|_{m} \leq|x|_{n} \leq\|x\|
$$

and

$$
\|x\|=\sup _{n}|x|_{n}=\lim _{n \rightarrow \infty}|x|_{n} .
$$


This last equation implies that the family of semi-norms, $\left\{\mid \cdot{ }_{n}: n \in \mathbb{N}_{0}\right\}$, is separating. We will term the metrisable topology generated by this family of semi-norms the local topology: with this topology, $Y$ is a separated locally convex topological vector space (TVS). By definition, a sequence $\left(x_{n}\right)$ converges to $x$ in the local topology if and only if $\left|x_{n}-x\right|_{m} \rightarrow 0$ as $n \rightarrow \infty$ for all $m$, i.e. if and only if

$$
P_{m} x_{n} \rightarrow P_{m} x \text { as } n \rightarrow \infty, \text { for all } m .
$$

We will see below that, unless $P_{n}=I$ for each $n, Y$ is not complete in the local topology. Let $X$ denote the Fréchet space that is the completion of $Y$ in the local topology. The linear operators $P_{n}$, which are continuous as mappings from $Y$ equipped with the local topology to $Y$ equipped with the norm topology, have unique extensions to continuous linear operators from $X$ to $Y$ [77. The equations (2.1) hold for the extended operators $P_{n}: X \rightarrow Y$, by the continuity of the operators $P_{n}$ and since $Y$ is dense in its completion $X$. Extending the definition of the seminorm $|\cdot|_{n}$ from $Y$ to $X$ using equation (2.4), clearly $\left\{|\cdot|_{n}: n \in \mathbb{N}_{0}\right\}$ generates the extension of the local topology from $Y$ to $X$.

Let $\hat{Y}$ and $\tilde{Y}$ denote the linear subspaces of $X$,

$$
\hat{Y}:=\left\{x \in X:\|x\|:=\sup _{n}|x|_{n}<\infty\right\}
$$

and

$$
\tilde{Y}:=\cup_{n \in \mathbb{N}_{0}} P_{n}(X)
$$

and note that, for every $n, \tilde{Y}=\cup_{m \geq n} P_{m}(X) \subset Y$ by (ii). Let $Y_{0}$ denote the norm-closure of $\tilde{Y}$. It follows from [23, Theorem 2.1 (ii)] and the completeness of $X$ that, equipped with the norm $\|\cdot\|, \hat{Y}$ is a Banach space with $Y$ and $Y_{0} \subset Y$ as closed subspaces.

Lemma 2.7. [74, Lemma 1.1.20] $Y_{0}=\left\{x \in Y: Q_{n} x \rightarrow 0\right.$ as $\left.n \rightarrow \infty\right\}$ so that $Y=Y_{0}$ iff $P_{n} \in L(Y)$ converges strongly to $I$ as $n \rightarrow \infty$.

Example 2.8 Let $Y$ and $\mathcal{P}$ be as in Example 2.4 Then $X=L_{\mathrm{loc}}^{p}\left(\mathbb{R}^{N}\right)$, $\tilde{Y}$ is the set of compactly supported functions $x \in L^{p}\left(\mathbb{R}^{N}\right), \hat{Y}=Y=L^{p}\left(\mathbb{R}^{N}\right)$, $Y_{0}=L^{p}\left(\mathbb{R}^{N}\right)$, if $1<p<\infty$, and

$$
Y_{0}=\left\{x \in L^{\infty}\left(\mathbb{R}^{N}\right): \underset{|s|>a}{\operatorname{ess} \sup }|x(s)| \rightarrow 0 \text { as } a \rightarrow \infty\right\}
$$

if $p=\infty$.

EXAMPLE 2.9 Let $B U C\left(\mathbb{R}^{N}\right) \subset B C\left(\mathbb{R}^{N}\right)$ denote the set of bounded uniformly continuous functions on $\mathbb{R}^{N}$, let $C_{L}\left(\mathbb{R}^{N}\right) \subset B U C\left(\mathbb{R}^{N}\right)$ denote the set of those $x \in C\left(\mathbb{R}^{N}\right)$ for which $x(s) \rightarrow x_{\infty}$ as $|s| \rightarrow \infty$, uniformly in $s$, for some constant $x_{\infty}$. Let $C_{0}\left(\mathbb{R}^{N}\right)$ denote the set of those $x \in C_{L}\left(\mathbb{R}^{N}\right)$ for which $x(s) \rightarrow 0$ as $|s| \rightarrow \infty$, and let $C_{C}\left(\mathbb{R}^{N}\right)$ denote the set of compactly supported continuous functions. Note that $C_{0}\left(\mathbb{R}^{N}\right), C_{L}\left(\mathbb{R}^{N}\right), B U C\left(\mathbb{R}^{N}\right)$ are all closed subspaces of $B C\left(\mathbb{R}^{N}\right)$, equipped with the usual norm.

Let $Y$ denote one of $C_{0}\left(\mathbb{R}^{N}\right), C_{L}\left(\mathbb{R}^{N}\right), B U C\left(\mathbb{R}^{N}\right)$ or $B C\left(\mathbb{R}^{N}\right)$ and let $\mathcal{P}$ be as defined in Example 2.5. Then $\hat{Y}=B C\left(\mathbb{R}^{N}\right), \tilde{Y}=C_{C}\left(\mathbb{R}^{N}\right)$ and $Y_{0}=C_{0}\left(\mathbb{R}^{N}\right)$. 
ExAmPle 2.10 For $x \in \ell^{\infty}$ and $k \in \mathbb{Z}$ let $x_{k} \in \ell^{\infty}$ be defined by $x_{k}(m)=$ $x(m-k), m \in \mathbb{Z}$. Call $x \in \ell^{\infty}$ almost periodic (e.g. [51, Definition 3.58]) if $\left\{x_{k}: k \in \mathbb{Z}\right\} \subset \ell^{\infty}$ is relatively compact. Let $\ell_{\mathrm{AP}}^{\infty} \subset \ell^{\infty}$ denote the set of almost periodic functions. Let $Y=\ell_{\mathrm{AP}}^{\infty}$ and define $\mathcal{P}$ as in Example 2.3, noting that $P_{n}\left(\ell_{\mathrm{AP}}^{\infty}\right) \subset \ell_{\mathrm{AP}}^{\infty}$ for every $n \in \mathbb{N}_{0}$. Then $\hat{Y}=\ell^{\infty}$ and $Y_{0}$ is a strict subspace of $Y$. In particular, if $y(m)=\exp (2 \pi i a m), m \in \mathbb{Z}$, with $a$ irrational, then $y \in Y$ (see e.g. 51. Lemma 3.64,Proposition 3.65]) but $\left\|P_{n} y-y\right\|=2$, for $n \in \mathbb{N}_{0}$, so that $y \notin Y_{0}$ by Lemma 2.7 .

We will also be interested in a third topology on $\hat{Y} \supset Y$, intermediate between the local and norm topologies. Given a positive null-sequence $a: \mathbb{N}_{0} \rightarrow(0, \infty)$, define

$$
|x|_{a}:=\sup _{n} a(n)|x|_{n} .
$$

Then $\left\{|\cdot|_{a}: a\right.$ is a positive null sequence $\}$ is a second separating family on $\hat{Y}$ and generates another separated locally convex topology on $\hat{Y}$ which, by analogy with [10, we will term the strict topology. For $\left(x_{n}\right) \subset \hat{Y}, x \in \hat{Y}$, we will write $x_{n} \stackrel{s}{\rightarrow} x$ if $x_{n}$ converges to $x$ in the strict topology, i.e. if $\left|x_{n}-x\right|_{a} \rightarrow 0$ as $n \rightarrow \infty$ for every null sequence $a$.

The topology we have called the strict topology is termed the $\beta$ topology in 23. Various properties of the $\beta /$ strict topology are shown in [23, Theorem 2.1], in large part adapting arguments from [10. The properties that we need for our arguments are summarised in the next lemma. As usual [77. we will call a set $S$ in a TVS bounded if it is absorbed by every neighbourhood of zero, totally bounded if, for every neighbourhood of zero, $U$, there exists a finite set $\left\{a_{1}, \ldots, a_{N}\right\}$ such that $S \subset \cup_{1 \leq j \leq N}\left(a_{j}+U\right)$, and compact if every open cover of $S$ has a finite subcover. Every totally bounded set is bounded $[\mathbf{7 7}$.

Lemma 2.11. (i) In $\hat{Y}$ the bounded sets in the strict topology and the norm topology are the same.

(ii) On every norm-bounded subset of $\hat{Y}$ the strict topology coincides with the local topology.

(iii) A sequence $\left(x_{n}\right) \subset \hat{Y}$ is convergent in the strict topology iff it is convergent in the local topology and is bounded in the norm topology, so that

$$
x_{n} \stackrel{s}{\rightarrow} x \quad \Leftrightarrow \quad \sup _{n}\left\|x_{n}\right\|<\infty \text { and } P_{m} x_{n} \rightarrow P_{m} x \text { as } n \rightarrow \infty, \text { for all } m .
$$

(iv) A norm-bounded subset of $\hat{Y}$ is closed in the strict topology iff it is sequentially closed.

(v) A sequence in $\hat{Y}$ is Cauchy in the strict topology iff it is Cauchy in the local topology and bounded in the norm topology.

(vi) Let $S \subset \hat{Y}$. Then the following statements are equivalent:

(a) $S$ is totally bounded in the strict topology.

(b) $S$ is norm-bounded and totally bounded in the local topology.

(c) Every sequence in $S$ has a subsequence that is Cauchy in the strict topology.

Proof. For (i)-(iv) see [23, Theorem 2.1]. Part (v) follows from (iii) on noting that, if $f_{j}: \mathbb{N} \rightarrow \mathbb{N}, j=1,2$, are such that $f=\left(f_{1}, f_{2}\right): \mathbb{N} \rightarrow \mathbb{N}^{2}$ is a bijection, then, defining $y_{n}:=x_{f_{1}(n)}-x_{f_{2}(n)},\left(x_{n}\right)$ is Cauchy iff $\left(y_{n}\right)$ is convergent to zero. 
To see (vi) note that if (a) holds then $S$ is bounded in the strict topology and so in the norm topology by (ii). Also, $S$ is totally bounded in the (coarser) local topology. Thus (b) holds. Conversely, if (b) holds, $U$ is a neighbourhood of zero in the strict topology, $M:=\sup _{x \in S}\|x\|$, and $B:=\{x:\|x\| \leq 2 M\}$, then, by (ii), there exists a neighbourhood of zero in the local topology, $U^{\prime}$, such that $U \cap B=U^{\prime} \cap B$. Further, there exists a finite set $\left\{s_{1}, \ldots, s_{N}\right\} \subset S$ such that $S \subset \bigcup_{1 \leq j \leq N}\left(s_{j}+U^{\prime}\right)$. It follows that

$$
S \subset \bigcup_{1 \leq j \leq N}\left(s_{j}+U^{\prime} \cap B\right)=\bigcup_{1 \leq j \leq N}\left(s_{j}+U \cap B\right) \subset \bigcup_{1 \leq j \leq N}\left(s_{j}+U\right) .
$$

Thus also (b) $\Rightarrow(\mathrm{a})$.

To see that (b) and (c) are equivalent note that, as the local topology is metrisable, $S$ is totally bounded in the local topology iff every sequence in $S$ has a subsequence that is Cauchy in the local topology. Further, by (v), a sequence is Cauchy in the strict topology iff it is Cauchy in the local topology and norm-bounded.

Note that it follows from (ii) that the linear operators on $Y$ that are bounded with respect to the strict topology (map bounded sets onto bounded sets) are precisely the members of $L(Y)$.

Let $E$ denote one of $\hat{Y}, Y$ and $Y_{0}$. When it is necessary to make a clear distinction we will denote the TVS consisting of $E$ (considered as a linear space) equipped with the strict topology by $(E, s)$ and will denote the TVS (and Banach space) consisting of $E$ with the norm topology as $(E,\|\cdot\|)$.

LEMMA 2.12. If $P_{n}=I$ for some $n$, then the local, strict, and norm topologies coincide on $\hat{Y}$. If $P_{n} \neq I$ for all $n$, then:

(a) on $\hat{Y}$ the local topology is strictly coarser than the strict topology which is strictly coarser than the norm topology; and

(b) $\hat{Y}$, equipped with the local topology, is not complete, while $\hat{Y}$ equipped with the strict topology is complete and non-metrisable.

Proof. It is easy to see that any set open in the local topology is open in the strict topology and that any set open in the strict topology is open in the norm topology. If $P_{n}=I$ for some $n$ then the converse statements clearly hold, as at least one of the semi-norms defining each topology coincides with the norm. Thus the topologies coincide.

If $P_{n} \neq I$ for any $n$ then there exists $\left(x_{n}\right)$ such that $\left\|Q_{n} x_{n}\right\|=1$ for all $n$. For all $m, P_{m} Q_{n} x_{n}=0$ for all sufficiently large $n$, by (ii). Clearly $Q_{n} x_{n} \nrightarrow 0$, but it follows from 2.6 that $Q_{n} x_{n} \stackrel{s}{\rightarrow} 0$ as $n \rightarrow \infty$. Thus the strict and norm topologies are distinct. To see that the local and strict topologies are distinct, note that $n Q_{n} x_{n}$ converges to zero in the local topology but $\left\|n Q_{n} x_{n}\right\|=n \rightarrow \infty$ so that, by 2.6, $n Q_{n} x_{n} \stackrel{s}{\rightarrow} 0$.

If $\hat{Y}$ equipped with the local topology were complete it would be a Fréchet space and it would follow from the open mapping theorem 82 applied to the identity operator that the local and norm topologies coincide.

Let $Y^{*}$ denote the completion of $\hat{Y}$ in the strict topology. Then $Y^{*} \subset X$, since $\hat{Y} \subset X$ and $X$ is complete in the coarser local topology. Suppose $Y^{*} \neq \hat{Y}$. 
Then there exists $x \in Y^{*}$ with $|x|_{n} \rightarrow \infty$ as $n \rightarrow \infty$. Let $b_{n}:=1 / 2 \max \left(1,|x|_{n}\right)$, $a_{n}:=1 / b_{n}$, and $a=\left(a_{0}, a_{1}, \ldots\right)$. Then $y \in Y^{*}$ and $|x-y|_{a}<1$ imply that $|y|_{n}>|x|_{n} / 2$ for all sufficiently large $n$, so that $\left\{y \in \hat{Y}:|x-y|_{a}<1\right\}=\varnothing$. This is a contradiction, for $\hat{Y}$ is dense in its completion.

By definition, $Y_{0}$ is the completion of $\tilde{Y}$ in the norm topology and we have seen that $Q_{n} x \rightarrow 0$ if $x \in Y_{0}$ in Lemma 2.7. The next lemma states corresponding results for the strict topology.

Lemma 2.13. For $y \in \hat{Y}, Q_{n} y \stackrel{s}{\rightarrow} 0$ as $n \rightarrow \infty$. Further $\hat{Y}$ is the completion of $\tilde{Y}$ in the strict topology and $Y=\hat{Y}$ iff $Y$ is sequentially complete in the strict topology.

Proof. If $y \in \hat{Y} \subset X$ then $P_{n} y \in \tilde{Y} \subset Y$ and $P_{m} P_{n} y=P_{m} y$ for all sufficiently large $n$. Further, by (i), $\left\|P_{n} y\right\| \leq\|y\|$. Thus, by Lemma 2.11 (iii), $P_{n} y \stackrel{s}{\rightarrow} y$. Thus the completion of $\tilde{Y}$ contains $\hat{Y}$ and in fact coincides with $\hat{Y}$ since $\hat{Y}$ is complete by Lemma 2.12 (ii). Since $\hat{Y}$ is complete, $Y \subset \hat{Y}$ is sequentially complete iff it is sequentially closed. But, since $P_{n} y \stackrel{s}{\rightarrow} y$ for every $y \in \hat{Y}$, this holds iff $Y=\hat{Y}$.

As usual, we will call a subset of a topological space relatively compact if its closure is compact. We will call a subset of a topological space relatively sequentially compact if every sequence in the subset has a subsequence converging to a point in the topological space.

Lemma 2.14. Let $S \subset Y$. Then $S$ is compact in $(Y, s)$ iff it is sequentially compact. Further,

$$
(a) \Leftrightarrow(b) \Rightarrow(c) \Leftrightarrow(d)
$$

where (a)-(d) are the statements:

(a) $S$ is relatively compact in the strict topology.

(b) $S$ is relatively sequentially compact in the strict topology.

(c) $S$ is totally bounded in the strict topology.

(d) $S$ is norm-bounded and $P_{n}(S)$ is relatively compact in the norm topology for each $n$.

If $(Y, s)$ is sequentially complete (i.e. $Y=\hat{Y})$ then $(a)-(d)$ are equivalent.

Proof. To show that compactness (relative compactness) of $S$ is equivalent to sequential compactness (relative sequential compactness) it is enough to show this in the strict topology restricted to $\bar{S}$, the closure in $(Y, s)$ of $S$. But, if $S$ is relatively sequentially compact or relatively compact then it is bounded and so $\bar{S}$ is bounded. But, by (ii) of Lemma 2.11, the strict topology coincides with the metrisable local topology on bounded sets, and in metric spaces compactness and sequential compactness coincide. Thus the first statement of the theorem holds and also (a) $\Leftrightarrow$ (b). That (b) implies (c), and the converse if $Y$ is sequentially complete, is immediate from (vi) of Lemma 2.11. If (c) holds then, also by (vi) of Lemma 2.11, $S$ is norm-bounded and every sequence in $S$ has a subsequence that is Cauchy in the strict topology. Since $P_{n}$ is continuous from $(\hat{Y}, s)$ to $(\hat{Y},\|\cdot\|)$ and $(\hat{Y},\|\cdot\|)$ is complete, this implies that $P_{n}(S)$ is relatively compact in the norm topology. Finally, suppose (d) holds and take an arbitrary bounded sequence $\left(x_{n}\right) \subset \hat{Y}$. Choose a subsequence $\left(x_{n}^{(1)}\right)$ such that $P_{1} x_{n}^{(1)}$ norm-converges as $n \rightarrow \infty$. From $\left(x_{n}^{(1)}\right)$ choose 
a subsequence $\left(x_{n}^{(2)}\right)$ such that $P_{2} x_{n}^{(2)}$ norm-converges, and so on. Then $\left(y_{n}\right)$, with $y_{n}:=x_{n}^{(n)}$, which is bounded and Cauchy in the local topology is Cauchy in the strict topology by Lemma 2.11(iv). Thus every sequence in $S$ has a subsequence that is Cauchy in the strict topology, so that, by Lemma 2.11 (vi), (c) holds.

EXAMPLE 2.15 As an important example of relative compactness in the strict topology, consider the situation of Examples 2.5 and 2.9 , where $\hat{Y}=Y=B C\left(\mathbb{R}^{N}\right)$ and $(Y, s)$ is just $Y$ with the standard strict topology of [10]. It follows from the equivalence of (a) and (d) in the above lemma and the Arzela-Ascoli theorem that $S \subset Y$ is relatively compact in $(Y, s)$ iff $S$ is bounded and equicontinuous. Recall that $S \subset Y$ is equicontinuous if

$$
\sup _{x \in S}|x(s)-x(t)| \rightarrow 0 \text { as } t \rightarrow s,
$$

for all $s \in \mathbb{R}^{N}$.

REMARK 2.16 As the following corollary of the above lemma already indicates, many of the results we obtain in the text will simplify and become more complete in the case that $P_{n} \in K(Y)$ for all $n$. We note that, by (ii), $P_{n}$ is compact for all $n$ if, for every $N, P_{n}$ is compact for some $n>N$.

Corollary 2.17. If $Y$ is sequentially complete in the strict topology (i.e. $Y=$ $\hat{Y}$ ) and $P_{n} \in K(Y)$ for all $n$, then $S \subset Y$ is relatively compact in the strict topology iff it is norm-bounded. 


\section{CHAPTER 3}

\section{Classes of Operators}

We have introduced already $L(Y)$ and $K(Y)$, the sets of linear operators that are, respectively, bounded and compact on $(Y,\|\cdot\|)$. We have noted that $L(Y)$ coincides with the set of linear operators that are bounded on $(Y, s)$. Let $C(Y)$ and $S(Y)$ denote the sets of those linear operators that are, respectively, continuous and sequentially continuous on $(Y, s)$. Thus $A \in S(Y)$ if and only if, for every sequence $\left(x_{n}\right) \subset Y$ and $x \in Y$,

$$
x_{n} \stackrel{s}{\rightarrow} x \quad \Rightarrow \quad A x_{n} \stackrel{s}{\rightarrow} A x .
$$

Let $S N(Y)$ denote the set of those linear operators that are sequentially continuous from $(Y, s)$ to $(Y,\|\cdot\|)$, so that $A \in S N(Y)$ iff

$$
x_{n} \stackrel{s}{\rightarrow} x \quad \Rightarrow \quad A x_{n} \rightarrow A x .
$$

We remark that the operators in $S(Y)$ and $S N(Y)$ are precisely those termed $s$-continuous and $s n$-continuous, respectively, in [6].

From standard properties of topological vector spaces $\mathbf{8 2}$, Theorems A6 and 1.30], and Lemma 2.12, it follows that $C(Y) \subset S(Y) \subset L(Y)$. In fact we have the following stronger result.

Lemma 3.1. $C(Y)=S(Y)$.

Proof. Let $C(\hat{Y}), S(\hat{Y})$ denote the sets of linear operators on $\hat{Y}$ that are, respectively, continuous and sequentially continuous. For $n \in \mathbb{N}_{0}$ let $Y_{n}$ denote the linear subspace of $Y$,

$$
Y_{n}:=\left\{x \in Y:|x|_{n}=0\right\}=\left\{x \in Y: P_{m} x=0,0 \leq m \leq n\right\} .
$$

Note that, by (ii), for every $m \in \mathbb{N}_{0}, Q_{n}(\hat{Y}) \subset Y_{m}$ for all sufficiently large $n$, and, for all $x \in Y,\left\|x-Q_{n} x\right\|=\left\|P_{n} x\right\| \leq|x|_{n}$. Thus Assumption $\mathrm{A}^{\prime}$ of 23 holds and it follows from [23, Theorem 3.7] that $C(\hat{Y})=S(\hat{Y})$.

By Lemma 2.13, the sequential closure of $Y \subset \hat{Y}$ in the strict topology is $\hat{Y}$. In Lemma 3.18 we will show that every $A \in S(Y)$ has an extension $\hat{A} \in S(\hat{Y})$ defined by $\hat{A} x=\lim _{n \rightarrow \infty} A P_{n} x$, where the limit exists in the strict topology. Then $\hat{A} \in C(\hat{Y})$ and $A=\left.\hat{A}\right|_{Y} \in C(Y)$.

In view of this lemma it holds that

$$
S N(Y) \subset C(Y)=S(Y) \subset L(Y) .
$$

As Lemmas 3.3 3.4 below clarify, in general $S N(Y)$ is a strict subset of $S(Y)$. The following example shows that $S(Y) \neq L(Y)$ in general, indeed that $A$ may be compact on $(Y,\|\cdot\|)$ but not sequentially continuous on $(Y, s)$. 
Example 3.2 Let $Y=\ell^{\infty}$ and $P_{n}$ be as in Example 2.2 Let $c_{\ell}^{+}$denote the set of those $x \in \ell^{\infty}$ for which $\lim _{m \rightarrow+\infty} x(m)$ exists. By the Hahn-Banach theorem a bounded linear functional $\ell_{+}: Y \rightarrow \mathbb{C}$ exists such that $\ell_{+}(x)=\lim _{m \rightarrow+\infty} x(m)$, $x \in c_{\ell}^{+}$. Define $A \in L(Y)$ by $A x=\ell_{+}(x) y, x \in Y$, where $y \in Y$ is non-zero and fixed. Then the range of $A$ is one-dimensional so that $A \in K(Y) \subset L(Y)$. However, defining $x=(\ldots, 1,1,1, \ldots)$ and $x_{n}=Q_{n} x, Q_{n} x \stackrel{s}{\rightarrow} 0$ as $n \rightarrow \infty$ but $A Q_{n} x=1$ for all $n$. Thus $A \notin S(Y)$.

The following lemmas provide alternative characterisations of the classes $S N(Y)$ and $S(Y)$ and shed some light on the relationship with $K(Y)$.

Lemma 3.3. $A \in S N(Y)$ iff $A \in L(Y)$ and $\left\|A Q_{n}\right\| \rightarrow 0$ as $n \rightarrow \infty$.

Proof. Suppose $A \in S N(Y)$. Then $A \in L(Y)$. To see that also $\left\|A Q_{n}\right\| \rightarrow 0$ as $n \rightarrow \infty$, suppose that this does not hold. Then there is a bounded sequence $\left(x_{n}\right) \subset Y$ such that $A Q_{n} x_{n} \nrightarrow 0$. But this is impossible as $Q_{n} x_{n} \stackrel{s}{\rightarrow} 0$, and hence $\left\|A Q_{n} x_{n}\right\| \rightarrow 0$ as $n \rightarrow \infty$, which is a contradiction.

For the reverse implication, take an arbitrary sequence $\left(x_{n}\right) \subset Y$ with $x_{n} \stackrel{s}{\rightarrow} 0$ as $n \rightarrow \infty$. Then $\left\|x_{n}\right\|$ is bounded and $\left\|P_{m} x_{n}\right\| \rightarrow 0$ as $n \rightarrow \infty$ for every $m$. Now, for every $m$ and $n$,

$$
\begin{aligned}
\left\|A x_{n}\right\| & \leq\left\|A P_{m} x_{n}\right\|+\left\|A Q_{m} x_{n}\right\| \\
& \leq\|A\|\left\|P_{m} x_{n}\right\|+\left\|A Q_{m}\right\| \sup _{n}\left\|x_{n}\right\|
\end{aligned}
$$

holds, where $\left\|A Q_{m}\right\|$ can be made as small as desired by choosing $m$ large enough, and $\left\|P_{m} x_{n}\right\|$ tends to zero as $n \rightarrow \infty$.

Lemma 3.4. $A \in S(Y)$ iff $A \in L(Y)$ and $P_{m} A \in S N(Y)$ for every $m$.

Proof. If $A \in S(Y)$ then $A \in L(Y)$. The rest trivially follows from

$$
A x_{n} \stackrel{s}{\rightarrow} 0 \text { as } n \rightarrow \infty \quad \Longleftrightarrow \quad\left\|P_{m} A x_{n}\right\| \rightarrow 0 \text { as } n \rightarrow \infty \forall m
$$

for every bounded operator $A$ and every bounded sequence $\left(x_{n}\right) \subset Y$.

Corollary 3.5. $A \in S(Y)$ iff $A \in L(Y)$ and $\left\|P_{m} A Q_{n}\right\| \rightarrow 0$ as $n \rightarrow \infty$ for every $m$.

ExAmple 3.6 Let $Y=L^{p}\left(\mathbb{R}^{N}\right)$ and $P_{n}$ be defined as in Example 2.4. Let $\kappa \in L^{1}\left(\mathbb{R}^{N}\right)$ and let $A$ be the linear integral operator, a so-called convolution operator, defined by

$$
A x(s)=\int_{\mathbb{R}^{N}} \kappa(s-t) x(t) d t, \quad s \in \mathbb{R}^{N} .
$$

Then, by Young's inequality, $A \in L(Y)$ with $\|A\| \leq\|\kappa\|_{1}$. Further, $A \in S(Y)$, since $\left\|P_{m} A Q_{n}\right\| \rightarrow 0$ as $n \rightarrow \infty$, for all $m$. To see this, note that, for every $m$, $P_{m} A Q_{n}=0$ for all sufficiently large $n$ if $\kappa \in C_{0}^{\infty}\left(\mathbb{R}^{N}\right)$, and then use the density of $C_{0}^{\infty}\left(\mathbb{R}^{N}\right)$ in $L^{1}\left(\mathbb{R}^{N}\right)$.

Lemma 3.7. $S(Y) \cap K(Y) \subseteq S N(Y)$, with equality if and only if $P_{n} \in K(Y)$ for all $n$. 
Proof. Suppose $A \in S(Y) \cap K(Y)$. Take an arbitrary sequence $\left(x_{n}\right) \subset Y$ with $x_{n} \stackrel{s}{\rightarrow} 0$ as $n \rightarrow \infty$. From $A \in S(Y)$ we conclude that $A x_{n} \stackrel{s}{\rightarrow} 0$ as $n \rightarrow \infty$. Since $\left\{x_{n}\right\}$ is bounded and $A$ is compact, we know that $\left\{A x_{n}\right\}$ is relatively compact, so every subsequence of $\left(A x_{n}\right)$ has a norm-convergent subsequence, where the latter has limit 0 since $A x_{n} \stackrel{s}{\rightarrow} 0$ as $n \rightarrow \infty$. Of course, this property ensures that $A x_{n}$ itself norm-converges to 0 .

To see when equality holds consider that, by (ii), it holds for every $m$ that $P_{m} Q_{n}=0$ for all sufficiently large $n$. Thus, by Lemma 3.3, $P_{m} \in S N(Y)$ for all $m$. So clearly $S N(Y) \not \subset K(Y)$ if $P_{m}$ is not compact for all $m$. If $P_{m}$ is compact for all $m$ and $A \in S N(Y)$ then, by Lemma 3.3 again, $A$ is the norm limit $\lim _{m \rightarrow \infty} A P_{m}$, with $A P_{m}$ compact, so that $A$ is compact and $S N(Y) \subset K(Y)$. Thus equality holds iff $P_{m} \in K(Y)$ for all $m$.

Recall that (e.g. 3) if $K \in K(Y)$ and $A_{n}$ converges strongly to $A$ then, since pointwise convergence is uniform on compact sets, $\left\|\left(A_{n}-A\right) K\right\| \rightarrow 0$. This and Lemma 3.7 have the following implication.

Lemma 3.8. If $P_{n}$ converges strongly to $I$ then $\left\|Q_{n} K\right\| \rightarrow 0$ as $n \rightarrow 0$ for all $K \in K(Y)$, while if $P_{n}^{*}$ converges strongly to $I^{*}\left(P_{n}^{*}\right.$ and $I^{*}$ the adjoints of $P_{n}$ and I) then $\left\|K Q_{n}\right\| \rightarrow 0$ as $n \rightarrow 0$ for all $K \in K(Y)$, so that $K(Y) \subset S N(Y)$.

Lemma 3.9. Let $A$ be a linear operator on $Y$. Then the following statements are equivalent.

(a) $A \in S N(Y)$.

(b) $A \in L(Y)$ and there is a neighbourhood of zero, $U$, in $(Y, s)$, for which $A(U)$ is norm-bounded, in fact for which $\sup _{x \in U}\left\|A Q_{n} x\right\| \rightarrow 0$ as $n \rightarrow \infty$.

(c) $A$ is a continuous mapping from $(Y, s)$ to $(Y,\|\cdot\|)$.

Proof. That continuity implies sequential continuity, so that (c) $\Rightarrow$ (a), is standard 82 .

Suppose that (a) holds. Then $A \in L(Y) \supset S N(Y)$. Moreover, by Lemma 3.3 . $\left\|A Q_{n}\right\| \rightarrow 0$ as $n \rightarrow \infty$. Choose positive integers $n_{1} \ll n_{2} \ll \ldots$ such that

$$
\left\|A Q_{j}\right\| \leq 4^{-m}, \quad j \geq n_{m},
$$

for $m \in \mathbb{N}$. Then, for $x \in Y, m \in \mathbb{N}$, and $n_{m} \leq j \leq n_{m+1}$, since $P_{n_{N}} Q_{j} x \stackrel{s}{\rightarrow} Q_{j} x$ as $N \rightarrow \infty$ and $A \in S N(Y)$,

$$
\begin{aligned}
\left\|A Q_{j} x\right\| & =\lim _{N \rightarrow \infty}\left\|A P_{n_{N}} Q_{j} x\right\| \\
& =\lim _{N \rightarrow \infty}\left\|A\left(Q_{j}-Q_{n_{N}}\right) x\right\| \\
& =\lim _{N \rightarrow \infty}\left\|\sum_{i=m+1}^{N-1} A\left(Q_{\tilde{n}_{i}}-Q_{\tilde{n}_{i+1}}\right) x\right\|,
\end{aligned}
$$

where $\tilde{n}_{i}:=n_{i}$, for $i>m+1$, and $\tilde{n}_{m+1}:=j$. Further, for $i \geq m+1$,

$$
\left\|A\left(Q_{\tilde{n}_{i}}-Q_{\tilde{n}_{i+1}}\right) x\right\|=\left\|A Q_{\tilde{n}_{i}} P_{\tilde{n}_{i+1}} x\right\| \leq 4^{1-i}\left\|P_{\tilde{n}_{i+1}} x\right\| .
$$

Now, define $n_{0}:=0$ and $a_{m}:=2^{-i}$, for $n_{i} \leq m<n_{i+1}, i \in \mathbb{N}_{0}$, and set $a:=$ $\left(a_{0}, a_{1}, \ldots\right)$ and $U:=\left\{x:|x|_{a}<1\right\}$. Then, from the above inequalities, we see that, 
for $x \in U, m \in \mathbb{N}$, and $n_{m} \leq j \leq n_{m+1}$, it holds that

$$
\left\|A Q_{j} x\right\| \leq \sum_{i=m+1}^{\infty} 4^{1-i}\left\|P_{\tilde{n}_{i+1}} x\right\| \leq \sum_{i=m+1}^{\infty} 4^{1-i} / a_{\tilde{n}_{i+1}} \leq 2^{3-m},
$$

since $a_{\tilde{n}_{i+1}} \geq a_{n_{i+1}}=2^{-(i+1)}$. Thus

$$
\sup _{x \in U}\left\|A Q_{j} x\right\| \rightarrow 0 \text { as } j \rightarrow \infty .
$$

Moreover, for $x \in U,\left\|P_{n_{1}} x\right\| \leq 1 / a_{n_{1}}=2$, so that

$$
\sup _{x \in U}\|A x\| \leq \sup _{x \in U}\left\|A P_{n_{1}} x\right\|+\sup _{x \in U}\left\|A Q_{n_{1}} x\right\| \leq 2\|A\|+4 .
$$

Since $U$ is a neighbourhood in $(Y, s)$, we have shown that (a) $\Rightarrow$ (b).

Now suppose that (b) holds, so that $A \in L(Y)$ and $A(U)$ is norm-bounded, with $U$ a neighbourhood of 0 in $(Y, s)$. To show that (c) holds it is enough to show that $A$ is continuous at 0 . But if $V$ is a neighbourhood of 0 in $(Y,\|\cdot\|)$ then $V$ contains $\lambda A(U)$, for some $\lambda>0$, and so $A^{-1}(V)$ contains $\lambda U$. Thus (b) $\Rightarrow(\mathrm{c})$.

Recall that $K(Y)$ stands for the set of compact operators on $Y$. Following $[\mathbf{7 4}$, $\S 1.1 .2]$, we denote the set of all $K \in L(Y)$ which are subject to

$$
\left\|K Q_{n}\right\| \rightarrow 0 \text { and } \quad\left\|Q_{n} K\right\| \rightarrow 0 \quad \text { as } \quad n \rightarrow \infty
$$

by $K(Y, \mathcal{P})$. Moreover, let $L(Y, \mathcal{P})$ refer to the set of all bounded linear operators $A$ on $Y$ such that $A K$ and $K A$ are in $K(Y, \mathcal{P})$ whenever $K \in K(Y, \mathcal{P})$. Both $K(Y, \mathcal{P})$ and $L(Y, \mathcal{P})$ are Banach subalgebras of $L(Y)$, and $K(Y, \mathcal{P})$ is a two-sided ideal in $L(Y, \mathcal{P})$. By definition, $L(Y, \mathcal{P})$ is the largest subalgebra of $L(Y)$ with that property. It is shown in [74, Theorem 1.1.9] that $L(Y, \mathcal{P})$ is inverse closed; that is, if $A \in L(Y, \mathcal{P})$ is invertible then $A^{-1} \in L(Y, \mathcal{P})$.

Lemma 3.10. An operator $A \in L(Y)$ is in $L(Y, \mathcal{P})$ iff for every $m \in \mathbb{N}_{0}$,

$$
\left\|P_{m} A Q_{n}\right\| \rightarrow 0 \quad \text { and } \quad\left\|Q_{n} A P_{m}\right\| \rightarrow 0 \quad \text { as } n \rightarrow \infty
$$

Proof. This is a straightforward computation. (See [74, Prop. 1.1.8].)

REMARK 3.11 Clearly the characterisations of $S(Y)$ and $S N(Y)$ in Lemma 3.3 and Corollary 3.5 bear a close resemblance to the definition of $K(Y, \mathcal{P})$ and the characterisation of $L(Y, \mathcal{P})$ in the above lemma, respectively. In particular, in the case that $Y$ is a Hilbert space and $P_{n}$ is self-adjoint, for each $n$, it holds that $A \in K(Y, \mathcal{P})(\in L(Y, \mathcal{P}))$ iff $A$ and $A^{*}$ are in $S N(Y)$ (in $S(Y)$ ), where $A^{*}$ denotes the adjoint of $A$.

The above characterisation also yields the following interesting result:

LEMma 3.12. For an operator $K \in L(Y, \mathcal{P})$, either both or neither of the two properties in (3.5) hold, so that $L(Y, \mathcal{P}) \cap S N(Y)=K(Y, \mathcal{P})$.

Proof. Suppose $K \in L(Y, \mathcal{P})$ and $\left\|K Q_{n}\right\| \rightarrow 0$ as $n \rightarrow \infty$. Then for all $m, n \in \mathbb{N}_{0}$,

$$
\left\|Q_{n} K\right\| \leq\left\|Q_{n} K P_{m}\right\|+\left\|Q_{n} K Q_{m}\right\| \leq\left\|Q_{n} K P_{m}\right\|+\left\|K Q_{m}\right\|
$$

holds, where $\left\|K Q_{m}\right\|$ can be made as small as desired by choosing $m$ large enough, and $\left\|Q_{n} K P_{m}\right\|$ tends to zero as $n \rightarrow \infty$. Consequently, also the second property holds in (3.5). By a symmetric argument we see that the second property implies the first. 
In analogy to Lemma 3.7 we have the following result.

LEMmA 3.13. $L(Y, \mathcal{P}) \cap K(Y) \subseteq K(Y, \mathcal{P})$, with equality if and only if $P_{n} \in K(Y)$ for all $n$.

Proof. From Corollary 3.5 and Lemma 3.10 we know that $L(Y, \mathcal{P}) \subseteq S(Y)$. Consequently,

$$
\begin{aligned}
L(Y, \mathcal{P}) \cap K(Y) & =L(Y, \mathcal{P}) \cap L(Y, \mathcal{P}) \cap K(Y) \\
& \subseteq L(Y, \mathcal{P}) \cap S(Y) \cap K(Y) \\
& \subseteq L(Y, \mathcal{P}) \cap S N(Y)=K(Y, \mathcal{P}),
\end{aligned}
$$

where we used Lemmas 3.7 and 3.12 for the last two steps. Moreover, if $P_{n} \in K(Y)$ for all $n$ and $K \in K(Y, \mathcal{P})$ then $P_{n} K \in K(Y)$ for all $n$ and $K=\lim P_{n} K \in K(Y)$. If $P_{n} \notin K(Y)$ for some $n$ then $P_{n}$ is contained in the difference of the two sets under consideration.

The above lemma has the following refinement in the case when both $P_{n}$ and its adjoint converge strongly to the identity.

Definition 3.14. 74 Call $\mathcal{P}$ perfect if $P_{n}$ converges strongly to $I$ and $P_{n}^{*}$ converges strongly to $I^{*}$.

Lemma 3.15. If $\mathcal{P}$ is perfect then $K(Y) \subset K(Y, \mathcal{P})$. If also $P_{n} \in K(Y)$ for every $n$, then $K(Y)=K(Y, \mathcal{P})$ and $L(Y)=L(Y, \mathcal{P})$.

Proof. That $K(Y) \subset K(Y, \mathcal{P})$ follows from Lemma 3.8, and that $K(Y, \mathcal{P}) \subset K(Y)$ if $\mathcal{P} \subset K(Y)$ follows from Lemma 3.13. Then $L(Y, \mathcal{P})=L(Y)$ is immediate from the definition of $L(Y, \mathcal{P})$.

The class of operators

$$
L_{0}(Y):=\left\{A \in L(Y): x \in Y_{0} \Rightarrow A x \in Y_{0}\right\}
$$

will turn out to be of particular interest to us. Recall that $Y_{0}$ is characterised by Lemma 2.7

Lemma 3.16. For $A \in L(Y)$, the condition $A \in L_{0}(Y)$ is equivalent to the strong convergence $Q_{n} A P_{m} \rightarrow 0$ as $n \rightarrow \infty$ for every fixed $m$.

Proof. Fix an arbitrary $m \in \mathbb{N}$. By Lemma 2.7, the strong convergence $Q_{n} A P_{m} \rightarrow 0$ as $n \rightarrow \infty$ is equivalent to $A P_{m} x \in Y_{0}$ for every $x \in Y$. Clearly, $A \in L_{0}(Y)$ implies $A P_{m} x \in Y_{0}$ for every $x \in Y$, since $P_{m} x \in Y_{0}$. The reverse implication follows from $P_{m} x \rightarrow x$ for every $x \in Y_{0}$, from the continuity of $A$, and the closedness of $Y_{0}$.

As an immediate consequence of Lemmas 3.10 and 3.16 we get the following.

Corollary 3.17. Every operator in $L(Y, \mathcal{P})$ maps $Y_{0}$ into $Y_{0}$, i.e. $L(Y, \mathcal{P}) \subset$ $L_{0}(Y)$.

We finish this discussion by noting that every $A \in S(Y)$ has a unique extension to $S(\hat{Y})$.

Lemma 3.18. Every $A \in S(Y)$ has a unique extension to an operator $\hat{A} \in S(\hat{Y})$, defined by

$$
\hat{A} x:=\lim _{n \rightarrow \infty} A P_{n} x, \quad x \in \hat{Y},
$$


where the limit is understood in the strict topology. It holds that $\|\hat{A}\|=\|A\|$, and if $A \in S N(Y), L(Y, \mathcal{P})$ or $K(Y, \mathcal{P})$, then $\hat{A} \in S N(\hat{Y}), L(\hat{Y}, \mathcal{P})$ or $K(\hat{Y}, \mathcal{P})$, respectively. Conversely, if $\hat{A} \in S(\hat{Y}), S N(\hat{Y}), L(\hat{Y}, \mathcal{P})$ or $K(\hat{Y}, \mathcal{P})$ and if $\hat{A}(Y) \subset$ $Y$, then $A:=\left.\hat{A}\right|_{Y} \in S(Y), S N(Y), L(Y, \mathcal{P})$ or $K(Y, \mathcal{P})$, respectively.

Proof. It is easy to see that every sequentially continuous linear operator on a TVS has a unique sequentially continuous extension to the sequential completion of the TVS. The obvious construction of this extension in our case is (3.7). For $x \in Y$, we have $A P_{n} x \stackrel{s}{\rightarrow} A x$ since $P_{n} x \stackrel{s}{\rightarrow} x$ and $A \in S(Y)$, so that $\left.\hat{A}\right|_{Y}=A$. Moreover, for $x \in \hat{Y}$,

$$
\|\hat{A} x\| \leq \sup _{n}\left\|A P_{n} x\right\| \leq\|A\| \sup _{n}\left\|P_{n} x\right\|=\|A\|\|x\|
$$

so that $\hat{A}$ is bounded and $\|\hat{A}\| \leq\|A\|$. Together with $\left.\hat{A}\right|_{Y}=A$, this gives $\|\hat{A}\|=$ $\|A\|$.

Now let us show that $\hat{A} \in S N(\hat{Y})$ if $A \in S N(Y)$. For every $x \in \hat{Y}$ and $n \in \mathbb{N}$, we have $P_{k} x \stackrel{s}{\rightarrow} x$, and therefore $A Q_{n} P_{k} x=\hat{A} Q_{n} P_{k} x \stackrel{s}{\rightarrow} \hat{A} Q_{n} x$ as $k \rightarrow \infty$ since $\hat{A}, Q_{n} \in S(\hat{Y})$. Thus, for $x \in \hat{Y}$ with $\|x\|=1$,

$$
\left\|\hat{A} Q_{n} x\right\| \leq \sup _{k}\left\|A Q_{n} P_{k} x\right\| \leq \sup _{k}\left\|A Q_{n} P_{k}\right\| \leq\left\|A Q_{n}\right\| \sup _{k}\left\|P_{k}\right\| \rightarrow 0
$$

as $n \rightarrow \infty$, by Lemma 3.3 , since $A \in S N(Y)$. Hence $\hat{A} \in S N(\hat{Y})$, by Lemma 3.3 again.

From the trivial equality $\left\|Q_{n} \hat{A} P_{m}\right\|=\left\|Q_{n} A P_{m}\right\|$, together with $\hat{A} \in S(\hat{Y})$, we get that $\hat{A} \in L(\hat{Y}, \mathcal{P})$ if $A \in L(Y, \mathcal{P})$, by Corollary 3.5 and Lemma 3.10 . Finally, it follows that $\hat{A} \in K(\hat{Y}, \mathcal{P})=L(\hat{Y}, \mathcal{P}) \cap S N(\hat{Y})$ if $A \in K(Y, \mathcal{P})=L(Y, \mathcal{P}) \cap S N(Y)$, by Lemma 3.12 .

\subsection{Compactness and Collective Compactness on $(Y, s)$}

A linear operator on a TVS is said to be compact if the image of some neighbourhood of zero is relatively compact. A linear operator is often said to be Montel if it has the weaker property that it maps bounded sets onto relatively compact sets. These properties coincide when the TVS is a normed space. Much of the familiar theory of compact operators on normed spaces generalises to compact operators on locally convex separated TVS's, for example the theory of Riesz [77. In particular, a compact operator has a discrete spectrum (as an element of the algebra $C(Y)$ ), whose only accumulation point is zero, and all non-zero points of the spectrum are eigenvalues. By contrast, as we will see below, the spectrum of a Montel operator may be much more complex.

Let $K S(Y)$ denote the set of compact operators on $(Y, s)$ and $M(Y)$ the set of Montel operators. Then it is standard (and clear) that $K S(Y) \subset M(Y) \subset L(Y)$ and $K S(Y) \subset C(Y)$. Also $K(Y) \subset M(Y)$, since bounded sets coincide in the strict and norm topologies and relatively compact sets in the norm topology are relatively compact in the strict topology. Thus, by Example 3.2 it may not hold that $M(Y) \subset S(Y)$. (A Venn diagram illustrating the various subsets of $L(Y)$ that we have introduced in this chapter is shown in Figure 3.1 below.) By Lemmas 2.11 and 2.14, an operator $A$ is in $M(Y)$ iff the image of every norm-bounded set is 
relatively sequentially compact in the strict topology. Operators with this property are termed sequentially compact with respect to the $(Y, s)$ topology in [23. The following two lemmas are useful characterisations of $M(Y)$ in the case when $Y$ is sequentially complete (which is the case when $Y=\hat{Y}$, by Lemma 2.13.

Lemma 3.19. If $Y=\hat{Y}$ then $A \in M(Y)$ iff $A \in L(Y)$ and $P_{m} A \in K(Y)$ for every $m$.

Proof. The lemma follows immediately from the equivalence of (a) and (d) in Lemma 2.14. This implies that $A \in M(Y)$ iff $A(S)$ is norm-bounded and $P_{n} A(S)$ is relatively compact in the norm topology, for every $n$ and every norm-bounded set $S$.

Remark 3.20 In the case $Y=L^{p}\left(\mathbb{R}^{N}\right)$ of Example 2.4 (in which case $\left.Y=\hat{Y}\right)$, an operator which satisfies $P_{m} A \in K(Y)$ and also $A P_{m} \in K(Y)$ for each $m$ is termed locally compact in [16, [51, 70, 74. In the case $Y=B C\left(\mathbb{R}^{N}\right)$ of Example 2.5 (in which again $Y=\hat{Y}$ ) an operator $A \in L(Y)$ is termed locally compact in [38] if it holds merely that $P_{m} A \in K(Y)$ for every $m$, i.e. by Lemma 3.19, if $A \in M(Y)$.

Lemma 3.21. If $A \in M(Y)$ then $A P_{n} \in K S(Y)$ for every $n$. Conversely, if $Y=\hat{Y}, A \in S(Y)$ and $A P_{n} \in M(Y)$ for every $n$, then $A \in M(Y)$.

Proof. By Lemma 3.3, $P_{n} \in S N(Y)$, and so, by Lemma 3.9, maps some neighbourhood in $(Y, s)$ to a bounded set in $(Y,\|\cdot\|)$. (In fact every neighbourhood in $(Y, s)$ is mapped to a bounded set.) Thus $A P_{n} \in K S(Y)$ if $A \in M(Y)$.

If $A P_{n} \in M(Y)$ for every $n$ then, by Lemma 3.19, $P_{m} A P_{n} \in K(Y)$ for every $m$ and $n$. If also $A \in S(Y)$ then, by Corollary 3.5 , $\left\|P_{m} A-P_{m} A P_{n}\right\| \rightarrow 0$ as $n \rightarrow \infty$, so that $P_{m} A \in K(Y)$ for every $m$. Thus $A \in M(Y)$ by Lemma 3.19 .

Many of the arguments we make in this text will deal with families of operators that have the following collective compactness property.

Definition 3.22. 23 We say that a set $\mathcal{K}$ of linear operators on $Y$ is uniformly Montel on $(Y, s)$ or is collectively sequentially compact on $(Y, s)$ if, for every bounded set $B, \cup_{K \in \mathcal{K}} K(B)$ is relatively compact in the strict topology.

REMARK 3.23 Note that, by Lemma 2.14. $\cup_{K \in \mathcal{K}} K(B)$ is relatively compact in the strict topology iff $\cup_{K \in \mathcal{K}} K(B)$ is relatively sequentially compact in the strict topology, i.e. iff, for every sequence $\left(K_{n}\right) \subset \mathcal{K}$ and $\left(x_{n}\right) \subset B,\left(K_{n} x_{n}\right)$ has a strictly convergent subsequence.

That being Montel on $(Y, s)$ is significantly weaker than being compact is very clear in the case when $P_{n}$ is compact for all $n$. The next two results follow from Corollary 2.17 (the first is also a corollary of Lemma 3.19).

Corollary 3.24. If $Y=\hat{Y}$ and $P_{n} \in K(Y)$ for every $n$ then $M(Y)=L(Y)$.

Corollary 3.25. If $Y=\hat{Y}$ and $P_{n} \in K(Y)$ for every $n$ then a set $\mathcal{K}$ of linear operators on $Y$ is uniformly Montel on $(Y, s)$ iff $\mathcal{K}$ is uniformly bounded.

REMARK 3.26 Some of our subsequent results will only apply to operators $A$ of the form $A=I+K$ with $K \in S(Y) \cap M(Y)$. It follows from Corollary 3.24 
that, if $Y=\hat{Y}$ and $P_{n} \in K(Y)$ for each $n$, then $A-I \in S(Y) \cap M(Y)$ whenever $A \in S(Y)$, so that every $A \in S(Y)$ can be written in this form.

$M(Y)$ is the set of operators which map bounded sets to relatively compact sets in $(Y, s)$, and we have seen in Lemma 3.9 that $S N(Y)$ is precisely the set of those operators that map some neighbourhood in $(Y, s)$ to a bounded set. On the other hand, $K S(Y)$ is the set of those operators that map some neighbourhood to a relatively compact set. Clearly, if $A \in M(Y) \cap S N(Y)$ then $A^{2} \in K S(Y)$. What is less clear is that $A \in K S(Y)$, which the next lemma shows.

Lemma 3.27. It always holds that $S(Y) \cap K(Y) \subset S N(Y) \cap M(Y)=K S(Y)$. If $P_{n} \in K(Y)$ for each $n$ then $S(Y) \cap K(Y)=S N(Y)=K S(Y)$.

Proof. We have seen already that $S(Y) \cap K(Y) \subset S N(Y)$ and $K(Y) \subset M(Y)$.

To show that $S N(Y) \cap M(Y)=K S(Y)$, suppose that $K \in K S(Y)$. Then, as noted already, it follows that $K \in M(Y)$. Since $K$ maps some neighbourhood of zero in $(Y, s)$ to a relatively compact set in $(Y, s)$, and relatively compact sets are bounded, it follows from Lemma 3.9 that $K \in S N(Y)$.

Suppose now that $K \in S N(Y) \cap M(Y)$. Then, by Lemma 3.9, there exists a neighbourhood of zero, $U$, in $(Y, s)$, for which $K(U)$ is norm bounded and $\sup _{x \in U}\left\|K Q_{n} x\right\| \rightarrow 0$ as $n \rightarrow \infty$. Let $a: \mathbb{N} \rightarrow(0, \infty)$ be a null sequence and let $V$ be the neighbourhood of zero, $V:=\left\{x \in U:|x|_{a}<1\right\}$. Then, for every $n, P_{n}(V) \subset Y$ is bounded so that, since $K \in M(Y)$, for every $n$, the image of every sequence in $V$ under the mapping $K P_{n}$ has an $s$-convergent subsequence. In particular, given a sequence $\left(x_{m}\right) \subset V$ we can construct a chain of subsequences $\left(x_{m}\right) \supset\left(x_{m}^{(1)}\right) \supset\left(x_{m}^{(2)}\right) \supset \cdots$ such that $K P_{j} x_{m}^{(n)}$ is $s$-convergent as $m \rightarrow \infty$ for $j=1, \ldots, n$. Then $K P_{j} x_{m}^{(m)}$ is $s$-convergent for every $j$, to a limit $y_{j} \in Y$. Now, for each $k, j_{1}, j_{2}$,

$$
\left\|P_{k}\left(y_{j_{1}}-y_{j_{2}}\right)\right\|=\lim _{m \rightarrow \infty}\left\|P_{k} K\left(Q_{j_{2}}-Q_{j_{1}}\right) x_{m}^{(m)}\right\| \leq \sup _{x \in V}\left\|K\left(Q_{j_{2}}-Q_{j_{1}}\right) x\right\| .
$$

Thus

$$
\left\|y_{j_{1}}-y_{j_{2}}\right\|=\sup _{k}\left\|P_{k}\left(y_{j_{1}}-y_{j_{2}}\right)\right\| \rightarrow 0
$$

as $j_{1}, j_{2} \rightarrow \infty$, so that, since $Y$ is a Banach space, for some $y \in Y, y_{j} \rightarrow y$ as $j \rightarrow \infty$. It follows that $K x_{m}^{(m)} \stackrel{s}{\rightarrow} y$ as $m \rightarrow \infty$, so that we have shown that $K(V)$ is relatively sequentially compact in $(Y, s)$ and so, by Lemma 2.14 , relatively compact, so that $K \in K S(Y)$. To see this last claim, note that $K(V) \subset K(U)$ is bounded and that, for every $k$ and $j$,

$$
\left\|P_{k}\left(K x_{m}^{(m)}-y\right)\right\| \leq\left\|P_{k}\left(K P_{j} x_{m}^{(m)}-y_{j}\right)\right\|+\left\|P_{k}\left(y_{j}-y\right)\right\|+\left\|P_{k} K Q_{j} x_{m}^{(m)}\right\|,
$$

which can be made as small as desired by choosing first $j$ and then $m$ sufficiently large.

The last sentence of the lemma follows immediately from Lemma 3.7 and from $S N(Y)=S(Y) \cap K(Y) \subset K(Y) \subset M(Y)$ and therefore $S N(Y) \cap M(Y)=S N(Y)$.

We finish the section with further examples of operators in $S(Y), K S(Y)$, and $M(Y)$. 


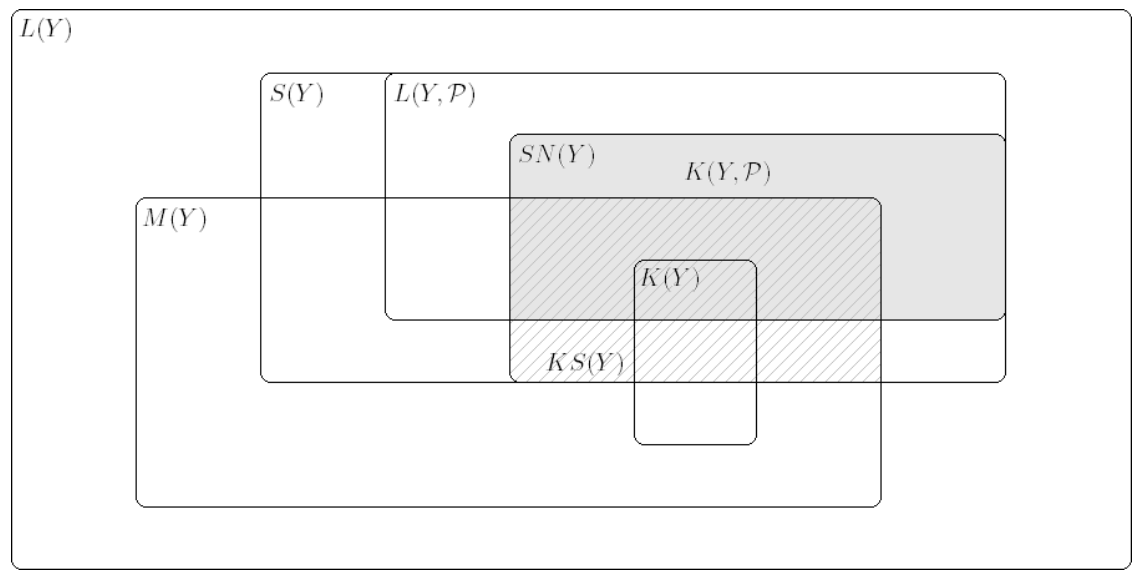

FIGURE 3.1. Venn diagram of the operator classes studied in this chapter. (The gray shaded area represents $K(Y, \mathcal{P})$, and the hatched area is $K S(Y)$.)

Example 3.28 Let $Y=B C\left(\mathbb{R}^{N}\right)$ and $P_{n}$ be defined as in Examples 2.5 and 2.15 so that $Y=\hat{Y}$ and $Y$ is sequentially complete in the strict topology. Suppose that $K$ is a linear integral operator on $Y$ of the form

$$
K x(s)=\int_{\mathbb{R}^{N}} k(s, t) x(t) d t, \quad s \in \mathbb{R}^{N},
$$

with $k(s, \cdot) \in L^{1}\left(\mathbb{R}^{N}\right)$ for every $s \in \mathbb{R}^{N}$. Then $[\mathbf{3 8}] K \in S(Y)$ iff

$$
\sup _{s \in \mathbb{R}^{N}} \int_{\mathbb{R}^{N}}|k(s, t)| d t<\infty
$$

and

$$
\int_{\mathbb{R}^{N}}\left|k(s, t)-k\left(s^{\prime}, t\right)\right| d t \rightarrow 0, \text { as } s^{\prime} \rightarrow s,
$$

for every $s \in \mathbb{R}^{N}$. Conditions 3.8 and 3.9 imply that $K$ maps bounded sets to bounded equicontinuous sets, that is to sets that are relatively compact in the strict topology (see Example 2.15). Thus $K \in M(Y) \cap S(Y)$ if (3.8) and (3.9) hold. Conditions (3.8) and (3.9) hold, in particular, if (as in Chapter 8$) \mathrm{K}$ is in the algebra generated by operators of multiplication by bounded continuous functions and operators of convolution by $L^{1}$ functions (as in Example 3.6), or in the closure of this algebra in $L(Y)$ with its norm topology. (Such operators are termed convolution-type operators in [74.)

ExAmPle 3.29 As a special case of the above example, suppose that $K$ is defined as in Example 3.6. Then 38 the spectrum of $K$ is $\{0\} \cup\left\{\hat{\kappa}(\xi): \xi \in \mathbb{R}^{N}\right\}$, where $\hat{\kappa} \in B C\left(\mathbb{R}^{N}\right)$ is the Fourier transform of $\kappa$. All non-zero points of the spectrum are eigenvalues $(\hat{\kappa}(\xi)$ has eigenfunction $x(s):=\exp (i \xi \cdot s))$. Since the spectrum of $K$ is not discrete, $K \in M(Y) \cap S(Y)$ but $K \notin K S(Y)$.

EXAmPle 3.30 (Cf. [5, 18.) As another special case of Example 3.28, consider the one-dimensional case $N=1$ with $K$ defined by

$$
K x(s)=\int_{0}^{1} \exp (i s t) x(t) d t, \quad s \in \mathbb{R} .
$$


Then $K=K P_{n}$ for all $n>1$, so that $K \in K S(Y)$ by Lemma 3.21. But $K \notin K(Y)$ as, defining $x_{n}(s)=\exp (-i n s), s \in \mathbb{R},\left(K x_{n}\right)$ has no norm-convergent subsequence since $K x_{n}(s) \rightarrow 0$ as $s \rightarrow \infty$ for every $n$ but $K x_{n}(n)=1$ for each $n$.

\subsection{Algebraic Properties}

We will find the algebraic properties collected in the following lemma useful. These are immediate from the definitions and Lemmas 3.9, 3.21 and 3.27.

Lemma 3.31. Let $A$ and $B$ be linear operators on $Y$. Then

$$
\begin{aligned}
A \in M(Y), \quad B \in L(Y) & \Rightarrow A B \in M(Y) \\
A \in S(Y), B \in M(Y) & \Rightarrow A B \in M(Y) \\
A \in L(Y), B \in S N(Y) & \Rightarrow A B \in S N(Y) \\
A \in S N(Y), \quad B \in S(Y) & \Rightarrow A B \in S N(Y) \\
A \in S N(Y), \quad B \in M(Y) & \Rightarrow A B \in K(Y) \\
A \in M(Y), \quad B \in S N(Y) & \Rightarrow A B \in K S(Y) \\
A \in K S(Y) & \Rightarrow A^{2} \in K(Y)
\end{aligned}
$$

$S(Y), S N(Y), M(Y)$, and $K S(Y)$ are all vector subspaces of $L(Y)$. It follows from the above lemma that they are all subalgebras of $L(Y)$, and that $S N(Y)$, $M(Y) \cap S(Y)$, and $K S(Y)$ are all (two-sided) ideals of $S(Y)$. Moreover, all these subalgebras are closed when endowed with the norm topology of $L(Y)$.

Lemma 3.32. $S(Y), S N(Y), M(Y)$, and $K S(Y)$ are all Banach subalgebras of $L(Y)$, with $S(Y)$ a unital subalgebra and $S N(Y), M(Y) \cap S(Y)$, and $K S(Y)$ two-sided ideals of $S(Y)$.

Proof. It only remains to show that each subalgebra is closed. If $A \in L(Y)$ is in the closure of $S N(Y)$ then, for every $B \in S N(Y)$ and every $n$,

$$
\left\|A Q_{n}\right\| \leq\left\|B Q_{n}\right\|+\left\|(B-A) Q_{n}\right\| \leq\left\|B Q_{n}\right\|+2\|B-A\| .
$$

Since $B$ can be chosen to make $\|B-A\|$ arbitrarily small and, by Lemma 3.3 . $\left\|B Q_{n}\right\| \rightarrow 0$ as $n \rightarrow \infty$ for every $B$, it follows that $\left\|A Q_{n}\right\| \rightarrow 0$ as $n \rightarrow \infty$ so that $A \in S N(Y)$. Thus $S N(Y)$ is closed.

Since $S N(Y)$ is closed it follows from Lemma 3.4 that $S(Y)$ is closed.

As $K(Y)$ is closed, it follows from Lemma 3.19 in the case $Y=\hat{Y}$ that $M(Y)$ is closed. In the general case, to see that $M(Y)$ is closed, suppose that $\left(A_{m}\right) \subset M(Y)$ and $A_{m} \rightrightarrows A \in L(Y)$. Let $\left(x_{n}\right)$ be a bounded sequence in $Y$. Then, by a diagonal argument as in the proof of Lemma 3.27, we can find a subsequence, denoted again by $\left(x_{n}\right)$, such that, for each $m$, there exists a $y_{m} \in Y$ such that $A_{m} x_{n} \stackrel{s}{\rightarrow} y_{m}$ as $n \rightarrow \infty$. Arguing as in the proof of Lemma 3.27, we can show that the sequence $\left(y_{m}\right)$ is Cauchy in $(Y,\|\|$.$) and so has a limit y \in Y$, and that $A x_{n} \stackrel{s}{\rightarrow} y$. This shows that the image of every bounded set under $A$ is relatively sequentially compact and so relatively compact in $(Y, s)$, by Lemma 2.14 i.e. $A \in M(Y)$.

By Lemma 3.27, $K S(Y)=M(Y) \cap S N(Y)$, being the intersection of two closed spaces, is closed itself. 
We will say that $A \in L(Y)$ is invertible if it is invertible in the algebra of linear operators on $Y$, i.e. if it is bijective. Automatically, by the open mapping theorem, it follows that $A^{-1} \in L(Y)$. An interesting question is whether $S(Y)=C(Y)$ is inverse closed, i.e. whether, if $A \in S(Y)$ is invertible, it necessarily holds that $A^{-1} \in S(Y)$. Since $(Y, s)$ is not barrelled [23, this question is not settled by standard generalisations of the open mapping theorem to non-metrisable TVS's 77. Indeed, it is not clear to us whether $S(Y)$ is inverse closed without further assumptions on $Y$. But we do have the following result which implies that $S(Y)$ is inverse closed in the case when $Y=\hat{Y}$ and $P_{n} \in K(Y)$ for each $n$.

Lemma 3.33. Suppose $A, B \in S(Y)$ are invertible and that $A^{-1} \in S(Y)$ and $A-B \in M(Y)$. Then $B^{-1} \in S(Y)$.

Proof. We have that $B^{-1}=D^{-1} A^{-1}$, where $D=I+C$ and $C=A^{-1}(B-A)$. By Lemma 3.31, $C \in S(Y) \cap M(Y)$. To show that $B^{-1} \in S(Y)$ we need only to show that $D^{-1} \in S(Y)$.

Suppose that $\left(x_{n}\right) \subset Y, x \in Y$, and $x_{n} \stackrel{s}{\rightarrow} x$. Let $y_{n}:=D^{-1} x_{n}$. By $(2.6)$, and since $D^{-1}=B^{-1} A \in L(Y),\left(x_{n}\right)$ and $\left(y_{n}\right)$ are bounded. For each $n$,

$$
y_{n}+C y_{n}=x_{n} .
$$

Since $C \in M(Y)$ there exists a subsequence $\left(y_{n_{m}}\right)$ and $y \in Y$ such that $x_{n_{m}}-$ $C y_{n_{m}} \stackrel{s}{\rightarrow} y$. From 3.10 it follows that $y_{n_{m}} \stackrel{s}{\rightarrow} y$. Since $C \in S(Y)$, it follows that $x_{n_{m}}-C y_{n_{m}} \stackrel{s}{\rightarrow} x-C y$. Thus $y=x-C y$, i.e. $y=D^{-1} x$. We have shown that $y_{n}=D^{-1} x_{n}$ has a subsequence strictly converging to $y=D^{-1} x$. By the same argument, every subsequence of $y_{n}$ has a subsequence strictly converging to $y$. Thus $D^{-1} x_{n} \stackrel{s}{\rightarrow} D^{-1} x$. So $D^{-1} \in S(Y)$.

Corollary 3.34. If $Y=\hat{Y}$ and $P_{n} \in K(Y)$ for all $n$ then $S(Y)$ is inverse closed.

Proof. If $Y=\hat{Y}$ and $P_{n} \in K(Y)$ for all $n$, and $A \in S(Y)$ is invertible, then $I-A \in M(Y)$ by Corollary 3.24 so that $A^{-1} \in S(Y)$ by the above lemma. 


\section{CHAPTER 4}

\section{Notions of Operator Convergence}

A component in the arguments to be developed is that one needs some notion of the convergence of a sequence of operators. For $\left(A_{n}\right) \subset L(Y), A \in L(Y)$, let us write $A_{n} \rightrightarrows A$ if $\left\|A_{n}-A\right\| \rightarrow 0$ (as $\left.n \rightarrow \infty\right)$ and $A_{n} \rightarrow A$ if $\left(A_{n}\right)$ converges strongly to $A$, in the strong operator topology induced by the norm topology on $Y$, i.e. if $A_{n} x \rightarrow A x$ for all $x \in Y$. Following [51, 74] we introduce also the following definition.

Definition 4.1. We say that a sequence $\left(A_{n}\right) \subset L(Y) \mathcal{P}$-converges to $A \in$ $L(Y)$ if, for all $K \in K(Y, \mathcal{P})$, both

$$
\left\|\left(A_{n}-A\right) K\right\| \rightarrow 0 \quad \text { and } \quad\left\|K\left(A_{n}-A\right)\right\| \rightarrow 0 \quad \text { as } n \rightarrow \infty .
$$

In this case we write $A_{n} \stackrel{\mathcal{P}}{\rightarrow} A$ or $A=\mathcal{P}-\lim A_{n}$.

The following lemma is a generalisation of Proposition 1.65 from [51. It shows that every $\mathcal{P}$-convergent sequence is bounded in $L(Y)$ and that, conversely, for a bounded sequence $\left(A_{n}\right)$ one has to check property 4.1 only for $K \in \mathcal{P}$ in order to guarantee $A_{n} \stackrel{\mathcal{P}}{\rightarrow} A$.

Lemma 4.2. Suppose $\left(A_{n}\right) \subset L(Y)$ and $A \in L(Y)$. Then $A_{n} \stackrel{\mathcal{P}}{\rightarrow} A$ iff $\left(A_{n}\right)$ is bounded in $L(Y)$ and, for all $m$,

$$
\left\|\left(A_{n}-A\right) P_{m}\right\| \rightarrow 0 \quad \text { and } \quad\left\|P_{m}\left(A_{n}-A\right)\right\| \rightarrow 0 \quad \text { as } n \rightarrow \infty .
$$

Proof. Suppose $\left(A_{n}\right)$ is bounded and 4.2 holds. Then, for all $m \in \mathbb{N}$ and all $K \in K(Y, \mathcal{P})$, one has

$$
\left\|K\left(A_{n}-A\right)\right\| \leq\|K\|\left\|P_{m}\left(A_{n}-A\right)\right\|+\left\|K Q_{m}\right\|\left\|A_{n}-A\right\|,
$$

where the first term tends to zero as $n \rightarrow \infty$, and the second one is as small as desired if $m$ is large enough. The first property of 4.1) is shown absolutely analogously.

Conversely, if 4.1 holds for all $K \in K(Y, \mathcal{P})$, then 4.2 holds for all $m \in \mathbb{N}$ since $\mathcal{P} \subset K(Y, \mathcal{P})$. It remains to show that $\left(A_{n}\right)$ is bounded.

Suppose the converse is true. Without loss of generality, we can suppose that $A=0$. Now we will successively define two sequences: $\left(m_{i}\right)_{i=1}^{\infty} \subset \mathbb{N}$ and $\left(n_{k}\right)_{k=0}^{\infty} \subset$ $\mathbb{N}_{0}$. We start with $m_{2} \gg m_{1}:=1$ and $n_{0}:=0$.

For every $k \in \mathbb{N}$, choose $n_{k} \in \mathbb{N}$ such that

$$
n_{k}>n_{k-1}, \quad\left\|A_{n_{k}}\right\|>k^{2}+3 \quad \text { and } \quad\left\|P_{m_{3 k-1}} A_{n_{k}}\right\|<1,
$$


the latter possible since $P_{m_{3 k-1}} \in K(Y, \mathcal{P})$ and $A_{n} \stackrel{\mathcal{P}}{\rightarrow} 0$. Then

$$
\left\|Q_{m_{3 k-1}} A_{n_{k}}\right\| \geq\left\|A_{n_{k}}\right\|-\left\|P_{m_{3 k-1}} A_{n_{k}}\right\|>k^{2}+3-1=k^{2}+2 .
$$

Take $u_{k} \in Y$ with $\left\|u_{k}\right\|=1$ and $\left\|Q_{m_{3 k-1}} A_{n_{k}} u_{k}\right\|>k^{2}+1$, and choose the next three elements of the sequence $\left(m_{i}\right)$ by $m_{3 k+2} \gg m_{3 k+1} \gg m_{3 k} \gg m_{3 k-1}$ such that $\left\|P_{m_{3 k}} Q_{m_{3 k-1}} A_{n_{k}} u_{k}\right\|>k^{2}$, which is possible by $[2.2$. Then

$$
\left\|P_{m_{3 k}} Q_{m_{3 k-1}} A_{n_{k}}\right\|>k^{2} \quad \text { for all } k \in \mathbb{N} \text {. }
$$

Now put

$$
K:=\sum_{j=1}^{\infty} \frac{1}{j^{2}} P_{m_{3 j+1}} Q_{m_{3 j-2}} .
$$

From $m_{i} \ll m_{i+1}$ for all $i \in \mathbb{N}$ we get that $P_{m_{3 k}} Q_{m_{3 k-1}} P_{m_{3 j+1}} Q_{m_{3 j-2}}$ equals $P_{m_{3 k}} Q_{m_{3 k-1}}$ if $k=j$ and 0 otherwise. Consequently,

$$
P_{m_{3 k}} Q_{m_{3 k-1}} K=\frac{1}{k^{2}} P_{m_{3 k}} Q_{m_{3 k-1}},
$$

and hence,

$$
\left\|K A_{n_{k}}\right\| \geq\left\|P_{m_{3 k}} Q_{m_{3 k-1}} K A_{n_{k}}\right\|=\left\|\frac{1}{k^{2}} P_{m_{3 k}} Q_{m_{3 k-1}} A_{n_{k}}\right\|>\frac{k^{2}}{k^{2}}=1
$$

for every $k \in \mathbb{N}$. On the other hand, $K \in K(Y, \mathcal{P})$, which implies $\left\|K A_{n}\right\| \rightarrow 0$ as $n \rightarrow \infty$. Contradiction.

The following is a simple but important example of $\mathcal{P}$-convergence that is fundamental to the application we study in Chapter 6.

EXAmple 4.3 Generalising Example 2.2 we suppose that $Y=\ell^{p}\left(\mathbb{Z}^{N}, U\right)$, for some $p \in[1, \infty]$ and $N \in \mathbb{N}$ where $U$ is some Banach space. The elements of $Y$ are of the form $x=(x(m))_{m \in \mathbb{Z}^{N}}$ with $x(m) \in U$ for every $m=\left(m_{1}, \ldots, m_{N}\right) \in \mathbb{Z}^{N}$ and we equip $Y$ with the usual norm. For $m \in \mathbb{Z}^{N}$, we define $|m|:=\max \left(\left|m_{1}\right|, \ldots,\left|m_{N}\right|\right)$ and put

$$
P_{n} x(m)= \begin{cases}x(m), & |m| \leq n, \\ 0, & |m|>n,\end{cases}
$$

for every $x \in Y$ and $n \in \mathbb{N}_{0}$. Similarly to Examples 2.2 and $2.4 \mathcal{P}=\left(P_{n}\right)$ satisfies (i) and (ii) with $N(m)=m$.

For $b=(b(m))_{m \in \mathbb{Z}^{N}} \in \ell^{\infty}\left(\mathbb{Z}^{N}, L(U)\right)$ define the multiplication operator $M_{b} \in$ $L(Y)$ by

$$
M_{b} x(m)=b(m) x(m), \quad m \in \mathbb{Z}^{N},
$$

for $x \in Y$, and note that $\left\|M_{b}\right\|=\|b\|$. It is a straightforward consequence of this equation and Lemma 4.2 that, for a sequence $b_{n} \in \ell^{\infty}\left(\mathbb{Z}^{N}, L(U)\right)$,

$$
\begin{aligned}
M_{b_{n}} \stackrel{\mathcal{P}}{\rightarrow} 0 & \Leftrightarrow \sup _{n}\left\|b_{n}\right\|<\infty \text { and } b_{n} \stackrel{s}{\rightarrow} 0 \\
& \Leftrightarrow \sup _{n}\left\|b_{n}\right\|<\infty \text { and }\left\|b_{n}(m)\right\| \rightarrow 0, \forall m \in \mathbb{Z}^{N} .
\end{aligned}
$$

In the above equation by $b_{n} \stackrel{s}{\rightarrow} 0$ we mean that $b_{n}$ converges to zero in the strict topology generated by the family $\mathcal{P}=\left(P_{n}\right)$, where we are here using the notation $P_{n}$ also to denote the operator on $\ell^{\infty}\left(\mathbb{Z}^{N}, L(U)\right)$ defined by 4.3 for $x \in \ell^{\infty}\left(\mathbb{Z}^{N}, L(U)\right)$. 
We have seen already that $S(Y)$ and $L(Y, \mathcal{P})$ are Banach subalgebras of $L(Y)$. Both are also closed with respect to $\mathcal{P}$-convergence.

Lemma 4.4. $S(Y)$ and $L(Y, \mathcal{P})$ are sequentially closed with respect to $\mathcal{P}$-convergence.

Proof. First suppose $\left(A_{n}\right) \subset S(Y), A \in L(Y)$ and $A_{n} \stackrel{\mathcal{P}}{\rightarrow} A$. Then, if $x_{n} \stackrel{s}{\rightarrow} 0$, for every $k$ and $m$, we have

$$
\left\|P_{k} A x_{n}\right\| \leq\left\|P_{k}\left(A-A_{m}\right)\right\| \sup _{n}\left\|x_{n}\right\|+\left\|P_{k} A_{m} x_{n}\right\| .
$$

But $\left\|P_{k} A_{m} x_{n}\right\| \rightarrow 0$ as $n \rightarrow \infty$ since $A_{m} \in S(Y)$, and $\left\|P_{k}\left(A-A_{m}\right)\right\|$ can be made as small as desired by choosing $m$ large. So we get $A x_{n} \stackrel{s}{\rightarrow} 0$, and therefore $A \in S(Y)$.

Similarly (see Proposition 1.1.17(a) in [74 for the details) we show that also $L(Y, \mathcal{P})$ is sequentially closed.

To make use of results from $2 \mathbf{2 3}$ we introduce also the notions of operator convergence used there. For $\left(A_{n}\right) \subset L(Y)$ and $A \in L(Y)$, let us write $A_{n} \stackrel{s}{\rightarrow} A$ if, for all $\left(x_{n}\right) \subset Y$,

$$
x_{n} \stackrel{s}{\rightarrow} x \quad \Rightarrow \quad A_{n} x_{n} \stackrel{s}{\rightarrow} A x .
$$

Call $\mathcal{A} \subset L(Y)$ s-sequentially compact if, for every sequence $\left(A_{n}\right) \subset \mathcal{A}$, there exists a subsequence $\left(A_{n_{m}}\right)$ and $A \in \mathcal{A}$ such that $A_{n_{m}} \stackrel{s}{\rightarrow} A$. Note that $A \stackrel{s}{\rightarrow} A$ holds iff $A \in S(Y)$. It follows that, if $\mathcal{A} \subset L(Y)$ is s-sequentially compact, then $\mathcal{A} \subset S(Y)$.

A more familiar and related notion of operator convergence is that of strong (or pointwise) convergence. For $\left(A_{n}\right) \subset L(Y), A \in L(Y)$, we will say that $\left(A_{n}\right)$ converges to $A$ in the strong operator topology on $(Y, s)$, and write $A_{n} \stackrel{S}{\rightarrow} A$, if

$$
A_{n} x \stackrel{s}{\rightarrow} A x, \quad x \in Y .
$$

Clearly, the $S$-limit is unique, that is $A_{n} \stackrel{S}{\rightarrow} A$ and $A_{n} \stackrel{S}{\rightarrow} B$ implies $A=B$. Hence also the $s$-limit and $\mathcal{P}$-limit are unique, by Lemma 4.5 and Corollary 4.8 below.

Clearly,

$$
A_{n} \rightarrow A \quad \Rightarrow \quad A_{n} \stackrel{S}{\rightarrow} A .
$$

The following lemmas explore further properties of and relationships between the notions of operator convergence we have introduced. We will exhibit this relationship through Example 4.6 .

Lemma 4.5. Suppose $\left(A_{n}\right) \subset L(Y), A \in L(Y)$. Then

$$
A_{n} \stackrel{s}{\rightarrow} A \quad \Rightarrow \quad A_{n} \stackrel{S}{\rightarrow} A \text { and } A \in S(Y) .
$$

Further, $A_{n} \stackrel{S}{\rightarrow} A$ as $n \rightarrow \infty$ iff $\left(A_{n}\right)$ is bounded and $P_{m}\left(A_{n}-A\right) \rightarrow 0$ as $n \rightarrow \infty$ for all $m \in \mathbb{N}$.

Proof. It is clear from the definitions that $A_{n} \stackrel{s}{\rightarrow} A$ implies $A_{n} \stackrel{S}{\rightarrow} A$. That $A_{n} \stackrel{s}{\rightarrow} A$ implies $A \in S(Y)$ is shown in [23, Lemma 3.1]. That $A_{n} \stackrel{S}{\rightarrow} A$ implies $P_{m}\left(A_{n}-A\right) \rightarrow 0$ is clear from (2.6), and that it also implies that $\left(A_{n}\right)$ is bounded is shown in [23, Lemma 3.3]. Conversely, if $\left(A_{n}\right)$ is bounded and $P_{m}\left(A_{n}-A\right) \rightarrow 0$ 
for each $m$, then, for every $x \in Y,\left(A_{n} x\right)$ is bounded and $P_{m}\left(A_{n} x-A x\right) \rightarrow 0$ for each $m$, so that $A_{n} x \stackrel{s}{\rightarrow} A x$ by 2.6 .

ExAmPle 4.6 Let $Y, P_{n}$ and the multiplication operator $M_{b}$ be defined as in Example 4.3 , and suppose that $\left(b_{n}\right) \subset \ell^{\infty}\left(\mathbb{Z}^{N}, L(U)\right)$. Then, extending the results of Example 4.3, we see that

$$
\begin{aligned}
M_{b_{n}} \rightrightarrows 0 & \Leftrightarrow\left\|b_{n}\right\|=\sup _{m \in \mathbb{Z}^{N}}\left\|b_{n}(m)\right\| \rightarrow 0, \\
M_{b_{n}} \stackrel{\mathcal{P}}{\rightarrow} 0 & \Leftrightarrow \sup _{n}\left\|b_{n}\right\|<\infty \text { and }\left\|b_{n}(m)\right\| \rightarrow 0, \quad \forall m \in \mathbb{Z}^{N}, \\
M_{b_{n}} \stackrel{s}{\rightarrow} 0 & \Leftrightarrow \quad M_{b_{n}} \stackrel{S}{\rightarrow} 0 \\
& \Leftrightarrow \sup _{n}\left\|b_{n}\right\|<\infty \text { and }\left\|b_{n}(m) x(m)\right\| \rightarrow 0, \quad \forall m \in \mathbb{Z}^{N}, x \in Y .
\end{aligned}
$$

Thus $M_{b_{n}} \stackrel{\mathcal{P}}{\rightarrow} 0$ requires that each component of $b_{n}$ converges to zero in norm, while $M_{b_{n}} \stackrel{s}{\rightarrow} 0$ requires that each component of $b_{n}$ converges strongly to zero. We have (cf. Corollary 4.14 below) that

$$
M_{b_{n}} \rightrightarrows 0 \Rightarrow M_{b_{n}} \stackrel{\mathcal{P}}{\rightarrow} 0 \Rightarrow M_{b_{n}} \stackrel{s}{\rightarrow} 0 \Leftrightarrow M_{b_{n}} \stackrel{S}{\rightarrow} 0 \Leftarrow M_{b_{n}} \rightarrow 0 .
$$

If $U$ is finite-dimensional, then $\stackrel{\mathcal{P}}{\rightarrow}, \stackrel{S}{\rightarrow}$ and $\stackrel{s}{\rightarrow}$ all coincide. If $p=\infty$, then $\rightarrow$ is equivalent to $\rightrightarrows$. If $1<p<\infty$ and $U$ is finite-dimensional, then $\rightarrow$ coincides with $\stackrel{\mathcal{P}}{\rightarrow}, \stackrel{S}{\rightarrow}$ and $\stackrel{s}{\rightarrow}$.

Lemma 4.7. Suppose $\left(A_{n}\right) \subset L(Y)$ is bounded, $A \in S(Y)$, and

$$
\left\|P_{m}\left(A_{n}-A\right)\right\| \rightarrow 0 \text { as } n \rightarrow \infty
$$

for each $m$. Then $A_{n} \stackrel{s}{\rightarrow} A$.

Proof. If the conditions of the lemma hold and $x_{n} \stackrel{s}{\rightarrow} x$ then $A x_{n} \stackrel{s}{\rightarrow} A x$ and, by (2.6), $\sup _{n}\left\|x_{n}\right\|<\infty$, so that $\left(A_{n} x_{n}\right)$ is bounded, and, for each $m$,

$$
\left\|P_{m}\left(A_{n} x_{n}-A x\right)\right\| \leq\left\|P_{m}\left(A_{n}-A\right) x_{n}\right\|+\left\|P_{m} A\left(x_{n}-x\right)\right\| \rightarrow 0
$$

as $n \rightarrow \infty$. Thus, by 2.6, $A_{n} x_{n} \stackrel{s}{\rightarrow} A x$.

As a corollary of Lemmas 4.2 and 4.7 we have

Corollary 4.8. Suppose $\left(A_{n}\right) \subset L(Y), A \in S(Y)$. Then

$$
A_{n} \stackrel{\mathcal{P}}{\rightarrow} A \quad \Rightarrow \quad A_{n} \stackrel{s}{\rightarrow} A .
$$

Let us say that $\mathcal{A} \subset L(Y)$ is $s$-sequentially equicontinuous if

$$
\left(A_{n}\right) \subset \mathcal{A}, x_{n} \stackrel{s}{\rightarrow} 0 \quad \Rightarrow \quad A_{n} x_{n} \stackrel{s}{\rightarrow} 0 .
$$

Clearly, if $\mathcal{A}$ is $s$-sequentially equicontinuous, then $\mathcal{A} \subset S(Y)$. The significance here of this definition is the following result taken from [23.

Lemma 4.9. Suppose $\left(A_{n}\right) \subset S(Y), A \in S(Y)$. Then $A_{n} \stackrel{s}{\rightarrow} A$ iff $A_{n} \stackrel{S}{\rightarrow} A$ and $\left\{A_{n}: n \in \mathbb{N}\right\}$ is s-sequentially equicontinuous.

Let us say that $\mathcal{A} \subset L(Y)$ is sequentially compact in the strong operator topology on $(Y, s)$ if, for every sequence $\left(A_{n}\right) \subset \mathcal{A}$, there exists $A \in \mathcal{A}$ and a subsequence 
$\left(A_{n_{m}}\right)$ such that $A_{n_{m}} \stackrel{S}{\rightarrow} A$. Then Lemma 4.9 and other observations made above imply the following corollary.

Corollary 4.10. Suppose $\mathcal{A} \subset L(Y)$. Then $\mathcal{A}$ is s-sequentially compact iff $\mathcal{A} \subset S(Y)$ and $\mathcal{A}$ is s-sequentially equicontinuous and sequentially compact in the strong operator topology on $(Y, s)$.

In the case that the strict and norm topologies coincide, in which case $C(Y)=$ $S(Y)=L(Y)$, it follows from Lemma 4.5, i.e. from the uniform boundedness theorem in Banach spaces, that if $\left(A_{n}\right) \subset L(Y)$ and $A_{n} \stackrel{S}{\rightarrow} A$ then $\left\{A_{n}: n \in \mathbb{N}\right\}$ is $s$-sequentially equicontinuous. In the case when these topologies do not coincide, in which case, by Lemma 2.12. $(Y, s)$ is not metrisable, other versions of the BanachSteinhaus theorem would apply [82, 77, if $(Y, s)$ were a Baire space or, more generally, a barrelled TVS, to give that $\left\{A_{n}: n \in \mathbb{N}\right\}$ is $s$-sequentially equicontinuous if $A_{n} \stackrel{S}{\rightarrow} A$ and $\left(A_{n}\right) \subset C(Y)$. But, by [23. Theorem 2.1], $(Y, s)$ is not barrelled unless the norm and strict topologies coincide. And in fact the following example makes it clear that a version of the Banach-Steinhaus theorem, enabling equicontinuity to be deduced from continuity and pointwise boundedness, does not always hold for $(Y, s)$ if the strict and norm topologies do not coincide.

ExAmple 4.11 Let $Y$ be defined as in Example 2.2. For $n \in \mathbb{N}$ define $A_{n} \in$ $L(Y)$ by $A_{n} x(m)=x(n)$, for $x \in Y, m \in \mathbb{Z}$. It is easy to see that $\left(A_{n}\right) \subset$ $C(Y) \subset L(Y)$, and clearly $\left\|A_{n}\right\| \leq 1$ so that $\left(A_{n}\right)$ is bounded. But $\left(A_{n}\right)$ is not $s$-sequentially equicontinuous as, defining $x_{n}(m)=1+\tanh (m-n), m \in \mathbb{Z}, n \in \mathbb{N}$, clearly $\left(x_{n}\right) \subset Y, x_{n} \stackrel{s}{\rightarrow} 0$, but $A_{n} x_{n}(0)=1$, so $A_{n} x_{n} \stackrel{s}{\rightarrow} 0$.

In the case that $Y$ satisfies an additional assumption, it is shown in $[23$ that a sequence $\left(A_{n}\right) \subset S(Y)$ that is convergent in the strong operator topology on $(Y, s)$ is $s$-sequentially equicontinuous. The additional assumption is the following one, in which $Y_{m} \subset Y$ is the subspace defined by (3.3):

Assumption A. For every $m \in \mathbb{N}$ there exists $n>m$ and $Q: Y \rightarrow Y_{m}$ such that

$$
\|x-Q x+y\| \leq \max \left(|x|_{n},\|y\|\right), \quad x \in Y, y \in Y_{n} .
$$

That Assumption A is satisfied in some applications is illustrated by the following example.

EXAMPLE 4.12 Suppose that $Y=B C\left(\mathbb{R}^{N}\right)$ and $P_{n}$ are defined as in Example 2.5. Then 4.11 holds with $n=m+2$ and $Q=Q_{m+1}$, for then $\|x-Q x+y\|=$ $\left\|P_{n-1} x+y\right\|=\max \left(\left\|P_{n-1} x\right\|,\|y\|\right) \leq \max \left(|x|_{n},\|y\|\right)$, for all $x \in Y$ and $y \in Y_{n}$.

Lemma 4.13. 23. Suppose that Assumption $A$ holds and that $\left(A_{n}\right) \subset S(Y)$, $A \in S(Y)$, and $A_{n} \stackrel{S}{\rightarrow} A$. Then $\left\{A_{n}: n \in \mathbb{N}\right\}$ is s-sequentially equicontinuous.

Combining Lemmas 4.13, 4.9, Corollary 4.8 and 4.87, we have the following result which shows that, when Assumption A holds, the convergence $\stackrel{s}{\rightarrow}$ is weaker than both ordinary strong convergence and $\mathcal{P}$-convergence. 
Corollary 4.14. Suppose that Assumption A holds and that $\left(A_{n}\right) \subset S(Y)$, $A \in S(Y)$. Then

$$
A_{n} \stackrel{\mathcal{P}}{\rightarrow} A \quad \Rightarrow \quad A_{n} \stackrel{s}{\rightarrow} A \quad \Leftrightarrow \quad A_{n} \stackrel{S}{\rightarrow} A \quad \Leftarrow \quad A_{n} \rightarrow A .
$$




\section{CHAPTER 5}

\section{Key Concepts and Results}

This chapter introduces the key concepts and develops the key results of the text. We first recall the concepts of invertibility at infinity and Fredholmness and

start to explore their inter-relation. Next, we summarise some main results from the abstract generalised collectively compact operator theory developed in $\mathbf{2 3}$ and from the abstract theory of limit operators $\mathbf{7 2}, \mathbf{7 4}, \mathbf{5 1}$. It then turns out that the collection of all limit operators of an operator $A \in S(Y)$ is subject to the constraints made in the operator theory of [23. Therefore we apply this theory and derive some new general results which can be used to study the invertibility of operators and their limit operators. We will illustrate the application of the results of this chapter throughout the remainder of the text.

\subsection{Invertibility at Infinity and Fredholmness}

Following [74, 51] we introduce the following definition.

Definition 5.1. An operator $A \in L(Y)$ is said to be invertible at infinity if there exist operators $B \in L(Y)$ and $T_{1}, T_{2} \in K(Y, \mathcal{P})$ such that

$$
A B=I+T_{1} \quad \text { and } \quad B A=I+T_{2} .
$$

Remark 5.2 If $A \in L(Y, \mathcal{P})$ then $A+K(Y, \mathcal{P})$ is invertible in the quotient algebra $L(Y, \mathcal{P}) / K(Y, \mathcal{P})$ iff $A$ is invertible at infinity with $B \in L(Y, \mathcal{P})$ in (5.1). Rabinovich et al. [74] call $A \in L(Y, \mathcal{P}) \mathcal{P}$-Fredholm when this is the case.

The following lemma gives some justification for the name 'invertible at infinity'.

LEMma 5.3. If $A \in L(Y)$ and (5.1) holds with $B \in L(Y)$ and $T_{1}, T_{2} \in S N(Y) \supset$ $K(Y, \mathcal{P})$, then there exist $B_{1}^{\prime}, B_{2}^{\prime} \in L(Y)$ and $n \in \mathbb{N}_{0}$ such that

$$
Q_{n} A B_{1}^{\prime}=Q_{n}=B_{2}^{\prime} A Q_{n} .
$$

If $B \in S(Y)$ then we can also choose $B_{1}^{\prime}, B_{2}^{\prime} \in S(Y)$. If $B, T_{1}, T_{2} \in L(Y, \mathcal{P})$ then also $B_{1}^{\prime}, B_{2}^{\prime}$ can be chosen in $L(Y, \mathcal{P})$.

Proof. Choose $m \in \mathbb{N}_{0}$ large enough that $\left\|T_{1} Q_{m}\right\|<1$ and $\left\|T_{2} Q_{m}\right\|<1$, and take an $n \in \mathbb{N}_{0}$ such that $Q_{n} Q_{m}=Q_{n}=Q_{m} Q_{n}$. From (5.1) we get $Q_{n} A B Q_{m}=$ $Q_{n}\left(I+T_{1} Q_{m}\right)$ and $B A Q_{n}=\left(I+T_{2} Q_{m}\right) Q_{n}$, proving (5.2) where we put $B_{1}^{\prime}:=$ $B Q_{m}\left(I+T_{1} Q_{m}\right)^{-1}$ and $B_{2}^{\prime}:=\left(I+T_{2} Q_{m}\right)^{-1} B$.

The two additional claims follow immediately from the Neumann series formula and the fact that $S(Y)$ and $L(Y, \mathcal{P})$ are Banach subalgebras of $L(Y)$. 
Recall that $A \in L(Y)$ is called semi-Fredholm if it has a closed range, $A(Y)$, and if one of the numbers

$$
\alpha(A):=\operatorname{dim}(\operatorname{ker} A) \quad \text { and } \quad \beta(A):=\operatorname{dim}(Y / A(Y))
$$

is finite, and that $A$ is called Fredholm if both $\alpha(A)$ and $\beta(A)$ are finite (in which case its range is automatically closed). In the latter case the index of $A$ is defined as $\alpha(A)-\beta(A)$.

The following theorem shows that, in some important cases, invertibility at infinity implies Fredholmness. Since $I \in S(Y)$, one possible choice of $C$ in this statement is $C=I$.

TheOrem 5.4. Suppose $A=C+K$, where $C \in L(Y)$ is invertible, with $C^{-1} \in$ $S(Y)$, and $K \in S(Y) \cap M(Y)$. Suppose further that (5.1) holds with $B \in L(Y)$ and $T_{1}, T_{2} \in S N(Y)$. Then $A$ is Fredholm.

Proof. We have from (5.1) that $C B+K B=I+T_{1}, B C+B K=I+T_{2}$, so that

$$
B=C^{-1}\left(I+T_{1}-K B\right), \quad B=\left(I+T_{2}-B K\right) C^{-1} .
$$

Using the first of these equations we see that

$$
A C^{-1}(I-K B)=\left(I+K C^{-1}\right)(I-K B)=I-K C^{-1} T_{1},
$$

and note that $K C^{-1} T_{1} \in K S(Y)$ by Lemma 3.31 and thus

$$
A C^{-1}(I-K B)\left(I+K C^{-1} T_{1}\right)=I-\left(K C^{-1} T_{1}\right)^{2},
$$

with $\left(K C^{-1} T_{1}\right)^{2} \in K(Y)$ by Lemma 3.31. Similarly,

$$
(I-B K) C^{-1} A=(I-B K)\left(I+C^{-1} K\right)=I-T_{2} C^{-1} K
$$

with $T_{2} C^{-1} K \in K(Y)$ by Lemma 3.31. We have constructed right and left regularisers for $A$, so $A$ is Fredholm.

The following is a corollary of the above result and Lemma 3.19. The last sentence follows from the observation that $A=I+(A-I)$ and that $P_{n}(A-I) \in$ $K(Y)$ if $A \in L(Y)$ and $P_{n} \in K(Y)$.

Corollary 5.5. If $A \in L(Y)$ is invertible at infinity and $A=I+K$, with $K \in S(Y)$ and $P_{n} K \in K(Y)$ for every $n$, then $A$ is Fredholm. In the case that $P_{n} \in K(Y)$ for all $n, A \in S(Y)$ is Fredholm if it is invertible at infinity.

In the case that $\mathcal{P}$ is perfect we will see in Lemma 5.13 that, conversely, Fredholmness implies invertibility at infinity. We will, in Chapter 6, also establish this result for the case $Y=\ell^{p}\left(\mathbb{Z}^{N}, U\right)$, for $p=1, \infty$, and a Banach space $U$, in which case $\mathcal{P}$ is not perfect.

\subsection{A Generalised Collectively Compact Operator Theory}

Following [23, Section 4], we let iso( $Y)$ denote the set of isometric isomorphisms on $Y$ and call a set $S \subset i s o(Y)$ sufficient if, for some $n \in \mathbb{N}$ it holds that, for every $x \in Y$ there exists $V \in S$ such that $2|V x|_{n} \geq\|x\|$. The following examples illustrate this definition: 
ExAmple 5.6 Let $Y=B C\left(\mathbb{R}^{N}\right)$ and $\mathcal{P}$ as in Example 2.5. Then both $S_{1}=$ $\left\{V_{k}: k \in \mathbb{R}^{N}\right\}$, where $V_{k}$ is the translation operator

$$
V_{k} x(s)=x(s-k), \quad s \in \mathbb{R}^{N},
$$

and $S_{2}=\left\{\Psi_{k}: k \in \mathbb{N}\right\}$, where

$$
\Psi_{k} x(s)=x(k s), \quad s \in \mathbb{R}^{N},
$$

are sufficient families of isometric isomorphisms on $Y$ where we can choose $n=1$ in both cases.

ExAmPle 5.7 Let $Y=L^{p}\left(\mathbb{R}^{N}\right)$ and $\mathcal{P}$ as in Example 2.4. If $p=\infty$ then both $S_{1}$ and $S_{2}$ from Example 5.6 are sufficient with $n=1$ for instance.

If $p<\infty$ then neither $S_{1}$ nor $S_{2}$ is sufficient. Although it is true that for every $x \in Y$ there are $n \in \mathbb{N}$ and $V_{k} \in S_{1}$ such that $2\left|V_{k} x\right|_{n} \geq\|x\|$, there is no universal $n \in \mathbb{N}$ which is large enough to guarantee this property for all $x \in Y$.

We say that an operator $A \in L(Y)$ is bounded below if

$$
\nu(A):=\inf _{\|x\|=1}\|A x\|>0 .
$$

In that case, we refer to $\nu(A)$ as the lower norm of $A$.

$A \in L(Y)$ is bounded below iff $A$ is injective and has a closed range. Indeed, necessity is obvious and sufficiency follows from Banach's theorem on the inverse operator saying that $A^{-1}: A(Y) \rightarrow Y$ acts boundedly on the range of $A$ if that is closed. Another elementary result on the lower norm is that it depends continuously on the operator; in particular, we have

$$
|\nu(A)-\nu(B)| \leq\|A-B\|
$$

for all $A, B \in L(Y)$.

If $A$ is invertible then $A$ is bounded below and $\nu(A)=1 /\left\|A^{-1}\right\|$. We will say that a set $\mathcal{A} \subset L(Y)$ is uniformly bounded below if every $A \in \mathcal{A}$ is bounded below and if there is a $\nu>0$ such that $\nu(A) \geq \nu$ for all $A \in \mathcal{A}$, that is

$$
\|A x\| \geq \nu\|x\|, \quad A \in \mathcal{A}, x \in Y .
$$

For $\mathcal{K} \subset L(Y)$, we abbreviate the set $\{I-K: K \in \mathcal{K}\}$ by $I-\mathcal{K}$.

In the following theorem we use the notation $\mathcal{K}^{\times}$to denote the set of all subsequences of sequences $\left(K_{1}, K_{2}, \ldots\right) \in \mathcal{K}_{1} \times \mathcal{K}_{2} \times \cdots$ for a fixed family of sets $\mathcal{K}_{1}, \mathcal{K}_{2}, \ldots \subset L(Y)$. This theorem is a slight strengthening of Theorems 4.1 and 4.4 in [23] (in 23] the condition (5.7) has ' $I-K_{n}$ bounded below' replaced by the weaker ' $I-K_{n}$ injective'), but an examination of the proof of Theorem 4.4 in [23] shows that this slightly stronger result follows by exactly the same argument.

THEOREM 5.8. Suppose that $Y=\hat{Y}, S \subset$ iso $(Y)$ is sufficient, $\mathcal{K}, \mathcal{K}_{1}, \mathcal{K}_{2}, \ldots \subset$ $L(Y)$, and that

(i) $\cup_{n \geq 1} \mathcal{K}_{n}$ is uniformly Montel on $(Y, s)$;

(ii) for every sequence $\left(K_{n}\right) \in \mathcal{K}^{\times}$, there exist a subsequence $\left(K_{n(m)}\right)$ and $K \in \mathcal{K}$ such that $K_{n(m)} \stackrel{s}{\rightarrow} K$ as $m \rightarrow \infty$;

(iii) for all $n \in \mathbb{N}$, it holds that $V^{-1} K V \in \mathcal{K}_{n}$ for all $K \in \mathcal{K}_{n}$ and $V \in S$;

(iv) $I-K$ is injective for all $K \in \mathcal{K}$. 
Then:

a) There is an $n_{0} \in \mathbb{N}$ such that $I-\cup_{n \geq n_{0}} \mathcal{K}_{n}$ is uniformly bounded below, i.e. there is a $\nu>0$ such that

$$
\|(I-K) x\| \geq \nu\|x\|, \quad K \in \mathcal{K}_{n}, n \geq n_{0}, x \in Y
$$

b) If, in addition, for every $K \in \mathcal{K}$, there exists a sequence $\left(K_{n}\right) \in \mathcal{K}^{\times}$such that $K_{n} \stackrel{s}{\rightarrow} K$ and all operators $I-K_{n}$ have the property

$$
I-K_{n} \text { bounded below } \Longrightarrow I-K_{n} \text { surjective, } \quad n=1,2, \ldots
$$

then all operators in $I-\mathcal{K}$ are invertible, and

$$
\sup _{K \in \mathcal{K}}\left\|(I-K)^{-1}\right\| \leq \nu^{-1} .
$$

The following special case of the above theorem, obtained by setting $\mathcal{K}_{1}=\mathcal{K}_{2}=$ $\cdots=\mathcal{K}$ in Theorem 5.8 is worth noting. We will say that a subset $\mathcal{A} \subset \mathcal{K} \subset L(Y)$ is $s$-dense in $\mathcal{K}$ if, for every $K \in \mathcal{K}$, there is a sequence $\left(K_{n}\right) \subset \mathcal{A}$ with $K_{n} \stackrel{s}{\rightarrow} K$.

Theorem 5.9. 23. Theorem 4.5] Suppose that $Y=\hat{Y}, S \subset$ iso( $Y)$ is sufficient and $\mathcal{K} \subset L(Y)$ has the following properties:

(i) $\mathcal{K}$ is uniformly Montel on $(Y, s)$;

(ii) $\mathcal{K}$ is s-sequentially compact;

(iii) $V^{-1} K V \in \mathcal{K}$ for all $K \in \mathcal{K}, V \in S$;

(iv) $I-K$ is injective for all $K \in \mathcal{K}$.

Then:

a) The set $I-\mathcal{K}$ is uniformly bounded below;

b) If in $I-\mathcal{K}$ there is an $s$-dense subset of surjective operators then all operators in $I-\mathcal{K}$ are surjective.

Note that in statement b), as in Theorem 5.8, all operators in $I-\mathcal{K}$ are consequently invertible, and their inverses are uniformly bounded by $1 / \nu$ where $\nu>0$ is a lower bound on all lower norms $\nu(I-K)$ with $K \in \mathcal{K}$ which exists by a).

\subsection{Limit Operators}

Following [74, Section 1.2], let $N \in \mathbb{N}$ and $\mathcal{V}=\left\{V_{k}\right\}_{k \in \mathbb{Z}^{N}}$ denote a group of linear isometries on $Y$ which are subject to

$$
V_{0}=I \quad \text { and } \quad V_{k} V_{m}=V_{k+m}, \quad k, m \in \mathbb{Z}^{N} .
$$

Moreover, we impose that $\mathcal{V}$ is compatible with $\mathcal{P}$ in the following sense:

$$
\forall m, n \in \mathbb{N} \quad \exists k_{0} \in \mathbb{N}: \quad P_{m} V_{k} P_{n}=0 \quad \text { if } \quad|k|>k_{0}
$$

and

Definition 5.10. 74 We will say that a sequence $h=(h(n))_{n=1}^{\infty} \subset \mathbb{Z}^{N}$ tends to infinity if $|h(n)| \rightarrow \infty$ as $n \rightarrow \infty$. If $h$ tends to infinity and $A \in L(Y)$ then

$$
A_{h}:=\mathcal{P}-\lim _{n \rightarrow \infty} V_{-h(n)} A V_{h(n)}
$$


is called the limit operator of $A$ with respect to the sequence $h$, provided the $\mathcal{P}$-limit exists.

From what we know about $\mathcal{P}$-convergence it follows that the limit operator $A_{h}$ is unique if it exists.

The operator spectrum $\sigma^{\mathrm{op}}(A):=\left\{A_{h}\right\}$ (see e.g. [51, 74 $)$ is the collection of all limit operators of $A$ where $h \subset \mathbb{Z}^{N}$ runs through all sequences tending to infinity such that $A_{h}$ exists. For certain operators $A$, invertibility at infinity, as specified in Definition 5.1. can be characterised in terms of properties of the operator spectrum (see Theorem 6.28 below). In turn, as we have seen in Corollary 5.5 for a large class of operators invertibility at infinity implies Fredholmness, so that Fredholmness can be determined by studying the operator spectrum; indeed, in some cases it is known that the operator spectrum also determines the index ([70, [71, [74, Section 2.7], 51. Section 3.3.1]). To establish the most complete results we need to restrict consideration to rich operators, where $A \in L(Y)$ is referred to as a rich operator if every sequence $h \subset \mathbb{Z}^{N}$ tending to infinity has an infinite subsequence $g$ such that the limit operator $A_{g}$ exists.

Example 5.11 Let $Y=\ell^{\infty}$ and define $\mathcal{P}$ as in Example 2.2 Define $\mathcal{V}=$ $\left\{V_{k}\right\}_{k \in \mathbb{Z}} \subset L(Y)$ by

$$
V_{k} x(m)=x(m-k), \quad m \in \mathbb{Z} .
$$

Then (5.8)-(5.10) hold for every $k_{0} \geq m+n$ and $n_{0} \geq m+k$. For $b \in \ell^{\infty}$ let $M_{b} \in L(Y)$ denote the multiplication operator defined by

$$
M_{b} x(m)=b(m) x(m), \quad m \in \mathbb{Z} .
$$

For $S \subset \mathbb{Z}$ let $\chi_{S} \in \ell^{\infty}$ denote the characteristic function of $S$. Define $a \in \ell^{\infty}$ by

$$
a(m):=\lfloor\sqrt{|m|}\rfloor \bmod 2, \quad m \in \mathbb{Z},
$$

where $\lfloor s\rfloor \leq s$ denotes the integer part of $s$, and set $A=M_{a}$. Then, where $B:=\left\{\chi_{\{n, \ldots,+\infty\}}, \chi_{\{-\infty, \ldots, n\}}: n \in \mathbb{Z}\right\}$,

$$
\sigma^{\mathrm{op}}(A)=\left\{0, I, M_{b}: b \in B\right\} .
$$

For example, if $h(n):=4 n^{2}+3$, then

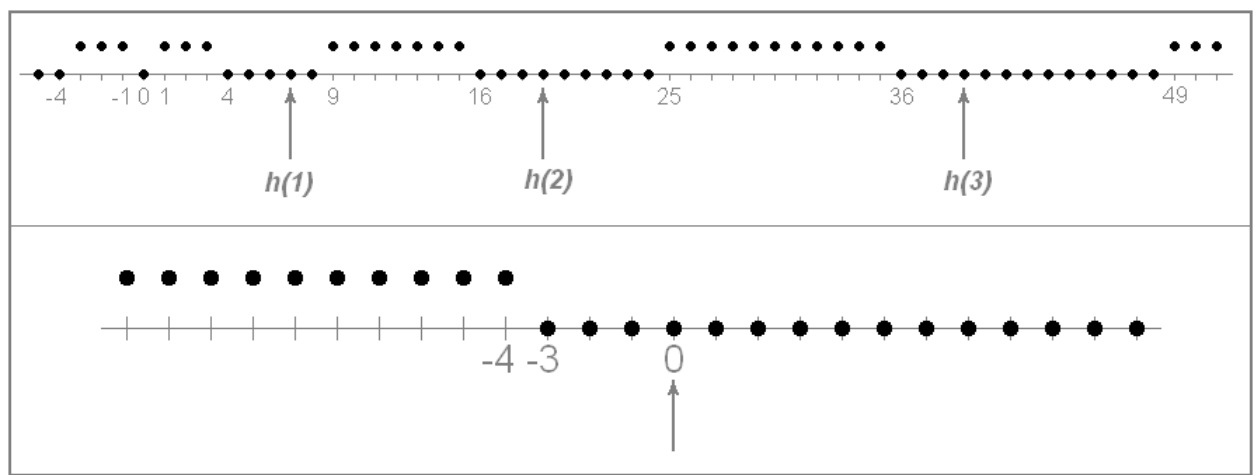

FiguRE 5.1. Functions $a$ (top) and $b=\chi_{\{-\infty, \ldots,-4\}}$ (below) from Example 5.11 


$$
\mathcal{P}-\lim _{n \rightarrow \infty} V_{-h(n)} A V_{h(n)}=M_{b}
$$

where $b:=\chi_{\{-\infty, \ldots,-4\}}$ (see Figure 5.1). The operator $A$ is rich; this can be seen directly or by applying Lemma 6.21 below.

The following theorem summarises and extends known results on the operator spectrum $\sigma^{\mathrm{op}}(A)$ and on the relationship between $A$ and its operator spectrum. Statements (i) and (ii) are from [72, (iii) and (iv) are from [74 and statements (v)-(vii) go back to [49, Section 3.3] and can also be found in [74, Section 1.2]. (Note that the proofs of (iii)-(vii) given in 4 49, 74] work for all $A \in L(Y)$, although the results state a requirement for $A \in L(Y, \mathcal{P})$ or make a particular choice of $Y$, and note also that (iv) is immediate from (ii) and (iii) and that (vii) is immediate from (ii), (v) and (vi), see [17.) Thus we include only a proof of (viii) and (ix), in which $Y_{0} \subset Y$ is as given in Chapter 2

For brevity, we introduce the notation

$$
\mathcal{T}(A):=\left\{V_{-k} A V_{k}: k \in \mathbb{Z}^{N}\right\}
$$

for the set of all translates of an operator $A \in L(Y)$.

Theorem 5.12. For every $A \in L(Y)$, the following statements hold.

(i) If $B \in \sigma^{\mathrm{op}}(A)$ then $\|B\| \leq\|A\|$.

(ii) If $B \in \sigma^{\mathrm{op}}(A)$ and $k \in \mathbb{Z}^{N}$ then also $V_{-k} B V_{k} \in \sigma^{\mathrm{op}}(A)$.

(iii) $\sigma^{\mathrm{oP}}(A)$ is sequentially closed with respect to $\mathcal{P}$-convergence.

(iv) If $B \in \sigma^{\mathrm{op}}(A)$ then $\sigma^{\mathrm{op}}(B) \subset \sigma^{\mathrm{oP}}(A)$.

(v) $A$ is rich iff $\mathcal{T}(A)$ is relatively $\mathcal{P}$-sequentially compact.

(vi) If $A$ is rich then $\sigma^{\mathrm{op}}(A)$ is $\mathcal{P}$-sequentially compact.

(vii) If $A$ is rich and $B \in \sigma^{\mathrm{op}}(A)$ then $B$ is rich.

(viii) If $B \in \sigma^{\mathrm{op}}(A)$ then $\|B x\| \geq \nu(A)\|x\|$ for $x \in Y_{0}$, so that $\nu(B) \geq \nu(A)$ if $Y=Y_{0}$.

(ix) If $B \in \sigma^{\mathrm{op}}(A) \cap L(Y, \mathcal{P})$ is invertible then $\nu(B) \geq \nu(A)$.

Proof. (viii) If $B \in \sigma^{\mathrm{op}}(A)$ then $B=A_{h}$ for some sequence $h \subset \mathbb{Z}^{N}$. For $m \in \mathbb{N}$ and every $x \in Y$ we have that

$$
\left\|V_{-h(n)} A V_{h(n)} P_{m} x\right\|=\left\|A V_{h(n)} P_{m} x\right\| \geq \nu(A)\left|\left\|\mid V_{h(n)} P_{m} x\right\|=\nu(A)\left\|P_{m} x\right\| .\right.
$$

Since $V_{-h(n)} A V_{h(n)} \stackrel{\mathcal{P}}{\rightarrow} B$, taking the limit as $n \rightarrow \infty$ we get

$$
\left\|B P_{m} x\right\| \geq \nu(A)\left\|P_{m} x\right\| \text {. }
$$

For $x \in Y_{0}$ we have, by Lemma 2.7, that $P_{m} x \rightarrow x$ as $m \rightarrow \infty$, so the result follows.

(ix) For $m, n \in \mathbb{N}$ and $x \in Y$,

$$
\begin{aligned}
\left\|P_{m} B^{-1} x\right\| & \leq\left\|P_{m} B^{-1} Q_{n} x\right\|+\left\|P_{m} B^{-1} P_{n} x\right\| \\
& \leq\left\|P_{m} B^{-1} Q_{n} x\right\|+\left\|B^{-1} P_{n} x\right\| .
\end{aligned}
$$

As $L(Y, \mathcal{P})$ is inverse closed [74, Theorem 1.1.9], we have that $B^{-1} \in L(Y, \mathcal{P})$, so that $\left\|P_{m} B^{-1} Q_{k}\right\| \rightarrow 0$ and $\left\|Q_{k} B^{-1} P_{n}\right\| \rightarrow 0$ as $k \rightarrow \infty$, the latter implying, by Lemma 2.7, that $B^{-1} P_{n} x \in Y_{0}$. Thus from 5.12 and (viii) we have that

$$
\begin{aligned}
\nu(A)\left\|P_{m} B^{-1} x\right\| & \leq \nu(A)\left\|P_{m} B^{-1} Q_{n} x\right\|+\nu(A)\left\|B^{-1} P_{n} x\right\| \\
& \leq \nu(A)\left\|P_{m} B^{-1} Q_{n} x\right\|+\left\|P_{n} x\right\| .
\end{aligned}
$$


Taking the limit first as $n \rightarrow \infty$ and then as $m \rightarrow \infty$, noting 2.2), we get that $\nu(A)\left\|B^{-1} x\right\| \leq\|x\|$. We have shown that $\nu(A)\|y\| \leq\|B y\|$, for all $y \in Y$, as required.

Within the subspace $L(Y, \mathcal{P})$ of $L(Y)$, for every fixed sequence $h$ tending to infinity, the mapping $A \mapsto A_{h}$ is compatible with all of addition, composition, scalar multiplication and passing to norm-limits [72]. That is, the equations

$$
\begin{aligned}
&(A+B)_{h}=A_{h}+B_{h},(A B)_{h}=A_{h} B_{h}, \\
&(\lambda A)_{h}=\lambda A_{h}, \quad\left(\lim _{m \rightarrow \infty} A^{(m)}\right)_{h}=\lim _{m \rightarrow \infty} A_{h}^{(m)}
\end{aligned}
$$

hold, in each case provided the limit operators on the right hand side exist. By definition, $L(Y, \mathcal{P})$ is a subalgebra of $L(Y)$. By Lemma 3.10, (5.10) implies that $\mathcal{V} \subset L(Y, \mathcal{P})$. Thus, if $A \in L(Y, \mathcal{P})$ then $\mathcal{T}(A) \subset L(Y, \mathcal{P})$, so that $\sigma^{\mathrm{op}}(A) \subset L(Y, \mathcal{P})$ by Lemma 4.4. Similarly, since by Lemma 3.32 $S(Y) \supset L(Y, \mathcal{P})$ is a subalgebra of $L(Y)$, if $A \in S(Y)$ then $V_{-k} A V_{k} \in S(Y)$ for all $k \in \mathbb{Z}^{N}$, so that $\sigma^{\text {op }}(A) \subset S(Y)$ by Lemma 4.4 . As a consequence of (5.13) together with a diagonal argument to see the closedness, the set of rich operators $A \in L(Y, \mathcal{P})$ is a Banach subalgebra of $L(Y, \mathcal{P})$.

We have at this point introduced all the main concepts that we will use in the rest of the text. The remainder of the text will in large part be focussed on establishing relationships between the following properties of an operator $A \in L(Y)$ for important operator classes:
(a) $A$ is invertible.
(b) $A$ is Fredholm.
(c) $A$ is invertible at infinity.
(d) All limit operators of $A$ are invertible and the inverses are uniformly bounded.
(e) All limit operators of $A$ are invertible.
(f) All limit operators of $A$ are injective.

Clearly, it always holds that $(\mathrm{a}) \Rightarrow(\mathrm{b})$ and that $(\mathrm{d}) \Rightarrow(\mathrm{e}) \Rightarrow(\mathrm{f})$. In the case that $\mathcal{P}$ is perfect (Definition 3.14) we have also the following result.

Lemma 5.13. If $\mathcal{P}$ is perfect then, for all $A \in L(Y),(b) \Rightarrow(c) \Rightarrow(d)$.

Proof. That $(\mathrm{b}) \Rightarrow(\mathrm{c})$ follows from Lemma 3.15. That $(\mathrm{c}) \Rightarrow(\mathrm{d})$ follows as in the proof of [74, Proposition 1.2.9] noting that this proof applies word for word with $L(Y, \mathcal{P})$ replaced by $L(Y)$, provided that we also replace 'P $\mathcal{P}$-Fredholm' by 'invertible at infinity'.

Thus we see that $(\mathrm{a}) \Rightarrow(\mathrm{b}) \Rightarrow(\mathrm{c}) \Rightarrow(\mathrm{d}) \Rightarrow(\mathrm{e}) \Rightarrow(\mathrm{f})$ in the case that $\mathcal{P}$ is perfect. Much of the remainder of the text will be concerned with establishing these implications also in cases when $\mathcal{P}$ is not perfect, and in investigating the converse. In particular, we will see in the next section that the generalised collectively compact operator theory introduced in the last section sheds some light on the relationships between (d), (e) and (f). Further, we have met in Section 5.1 conditions on $A$ which ensure that $(\mathrm{c}) \Rightarrow(\mathrm{b})$. 
One case in which the connection between the properties (5.14) is evident is the case in which $A$ is self-similar. Here, following [17] call $A \in L(Y)$ self-similar if $A \in \sigma^{\mathrm{op}}(A)$ and, generalising [58], call $A \in L(Y)$ recurrent if, for every $B \in \sigma^{\mathrm{op}}(A)$, it holds that $\sigma^{\mathrm{op}}(B)=\sigma^{\mathrm{op}}(A)$. It is immediate from these definitions and Theorem 5.12 (iv) that if $A$ is recurrent then all limit operators of $A$ are self-similar and recurrent. In the case when $A$ is self-similar, the following relationship between the properties (5.14) (a)-(e) of $A$ is immediate from the definitions and Lemma 5.13 .

COROLLARY 5.14. If $\mathcal{P}$ is perfect and $A \in L(Y)$ is self-similar then all of (a)-(e) in (5.14) are equivalent.

That self-similar operators exist, indeed are ubiquitous, is clear from the following lemma. For completeness, we include the proof which is a variation on the proof of [17, Proposition 3.7]. Proposition 3.7 in [17] is the same statement as Lemma 5.15, but proved only for the special case when $Y=\ell^{p}(\mathbb{Z}, U)$, for some $p \in[1, \infty]$ and some Banach space $U$.

Lemma 5.15. If $A \in L(Y)$ is rich then some $B \in \sigma^{\mathrm{op}}(A)$ is self-similar and recurrent.

Proof. If $B \in \sigma^{\mathrm{op}}(A)$ then $\sigma^{\mathrm{op}}(B) \subset \sigma^{\mathrm{op}}(A)$ (Theorem 5.12 (iv)) and $\sigma^{\mathrm{op}}(B)$ is non-empty since, by Theorem 5.12 (vii), $B$ is rich. Further, if $B$ is recurrent then every $C \in \sigma^{\mathrm{op}}(B) \subset \sigma^{\mathrm{op}}(A)$ is recurrent and self-similar. Thus it is enough to show that there exists a $B \in \sigma^{\mathrm{op}}(A)$ which is recurrent.

Let

$$
\mathcal{A}:=\left\{\sigma^{\mathrm{op}}(B): B \in \sigma^{\mathrm{op}}(A)\right\} .
$$

By Theorem 5.12 (iv) this is a partially ordered set, equipped with the order ' $\supseteq$ '. Note further that $\sigma^{\mathrm{op}}(B)$ is a maximal element in this partially ordered set iff $B$ is recurrent. So it remains to show the existence of a maximal element. But this follows from Zorn's lemma if we can show that every totally ordered subset of $\mathcal{A}$ has an upper bound.

So let $\mathcal{B}$ be a totally ordered subset of $\mathcal{A}$, i.e.

$$
\mathcal{B}=\left\{\sigma^{\mathrm{op}}(B): B \in \sigma\right\}
$$

where $\sigma \subseteq \sigma^{\mathrm{op}}(A)$ is such that, for any two $B_{1}, B_{2} \in \sigma$, we either have $\sigma^{\mathrm{op}}\left(B_{1}\right) \supseteq$ $\sigma^{\mathrm{op}}\left(B_{2}\right)$ or $\sigma^{\mathrm{op}}\left(B_{2}\right) \supseteq \sigma^{\mathrm{op}}\left(B_{1}\right)$. On $X:=\sigma^{\mathrm{op}}(A)$ define the following family of seminorms. Let

$$
\varrho_{2 n-1}(T):=\left\|P_{n} T\right\|, \quad \varrho_{2 n}(T):=\left\|T P_{n}\right\|,
$$

for $n=1,2, \ldots$ and every $T \in X$, and denote the topology that is generated on $X$ by $\left\{\varrho_{1}, \varrho_{2}, \ldots\right\}$ by $\mathcal{T}$. Note that, since $\mathcal{T}$ is generated by a countable family of seminorms, the topological space $(X, \mathcal{T})$ is metrisable, so that sequential compactness in $(X, \mathcal{T})$ coincides with compactness. Further, by Lemma 4.2 and since (Theorem 5.12(i)) $\|T\| \leq\|A\|$ for every $T \in X$, convergence in $(X, \mathcal{T})$ is equivalent to $\mathcal{P}$-convergence in $X$. Therefore, by Theorem 5.12(vi)-(vii), $X$ itself and all elements of $\mathcal{B}$ are compact sets in $(X, \mathcal{T})$.

Now put $\Sigma:=\cap_{B \in \sigma} \sigma^{\mathrm{op}}(B)=\cap_{C \in \mathcal{B}} C$. It follows, since $\mathcal{B}$ is totally ordered and each element of $\mathcal{B}$ is compact (see the proof of [17, Proposition 3.7] for details) 
that $\Sigma$ is non-empty. But clearly, by Theorem 5.12 (iv) again, for every $T \in \Sigma$, $\sigma^{\mathrm{op}}(T) \in \mathcal{A}$ is an upper bound of the chain $\mathcal{B}$.

We will see an application of this lemma, taken from 17, at the end of Section 6.3 , and we will meet concrete examples of self-similar operators in Section 6.1 and Chapter 7.

\subsection{Collective Compactness and the Operator Spectrum}

We continue to suppose that $\mathcal{V}$ satisfies the constraints (5.8)-(5.10) introduced in Section 5.3 This being the case, Theorem 5.12 shows how nicely the operator spectrum fits the conditions made on the set $\mathcal{K}$ in Theorem 5.9 if we put $S:=\mathcal{V}$. Indeed, property (iii) of Theorem 5.9 is then guaranteed by Theorem 5.12 (ii). Moreover, if the operator under consideration is rich and in $S(Y)$ then, by Theorem 5.12 (vi), its operator spectrum is $\mathcal{P}$-sequentially compact, and hence $s$-sequentially compact by Corollary 4.8 (recall that we have just seen after Theorem 5.12 that if $A \in S(Y)$ then $\sigma^{\text {op }}(A) \subset S(Y)$ ).

Bearing in mind these observations, we now apply Theorem 5.9 to the operator spectrum of an operator $A \in L(Y)$. We set $K:=I-A$ and apply Theorem 5.9 with

$$
\mathcal{K}:=\sigma^{\mathrm{op}}(K)=I-\sigma^{\mathrm{op}}(A) \quad \text { so that } \quad I-\mathcal{K}=\sigma^{\mathrm{op}}(A),
$$

noting that $K$ is rich iff $A$ is rich.

Theorem 5.16. Suppose $Y=\hat{Y}, A=I-K \in S(Y)$ is rich, $\mathcal{V}$ is sufficient, $\sigma^{\mathrm{op}}(K)$ is uniformly Montel on $(Y, s)$, and all the limit operators of $A$ are injective. Then $\sigma^{\mathrm{op}}(A)$ is uniformly bounded below. If, moreover, there is an s-dense subset of surjective operators in $\sigma^{\mathrm{op}}(A)$ then all elements of $\sigma^{\mathrm{op}}(A)$ are invertible and their inverses are uniformly bounded.

We can express the condition that $\sigma^{\mathrm{op}}(K)$ be uniformly Montel on $(Y, s)$ more directly in terms of properties of the operator $K$. This is the content of the next lemma, for which we introduce the following definition: call a sequence $\left(A_{k}\right)_{k \in \mathbb{Z}^{N}} \subset$ $L(Y)$ asymptotically Montel on $(Y, s)$ if, for every sequence $h=(h(n))_{n=1}^{\infty} \subset \mathbb{Z}^{N}$ tending to infinity and every bounded sequence $\left(x_{n}\right) \subset Y$, it holds that $A_{h(n)} x_{n}$ has a strictly converging subsequence.

Lemma 5.17. If $K \in L(Y)$ and the sequence $\left(V_{-k} K V_{k}\right)_{k \in \mathbb{Z}^{N}}$ is asymptotically Montel on $(Y, s)$, then $\sigma^{\mathrm{op}}(K)$ is uniformly Montel. Conversely, if $K$ is rich and $\sigma^{\mathrm{op}}(K)$ is uniformly Montel then $\left(V_{-k} K V_{k}\right)_{k \in \mathbb{Z}^{N}}$ is asymptotically Montel.

Proof. Suppose $\left(V_{-k} K V_{k}\right)_{k \in \mathbb{Z}^{N}}$ is asymptotically Montel and pick any sequence $\left(K_{n}\right)_{n \in \mathbb{N}} \subset \sigma^{\mathrm{op}}(K)$. Then, by definition of the operator spectrum and $\mathcal{P}$-convergence, for every $n \in \mathbb{N}$ we can find $h(n) \in \mathbb{Z}^{N}$ with $|h(n)| \geq n$ and

$$
\left\|P_{k}\left(K_{n}-V_{-h(n)} K_{n} V_{h(n)}\right)\right\| \leq \frac{1}{n}, \quad 1 \leq k \leq n .
$$

Now choose any $\left(x_{n}\right) \subset Y$ with $\left\|x_{n}\right\| \leq 1$. Then, as $\left(V_{-k} K V_{k}\right)_{k \in \mathbb{Z}^{N}}$ is asymptotically Montel and $h$ tends to infinity, $V_{-h(n)} K_{n} V_{h(n)} x_{n}$ has a strictly convergent subsequence. On the other hand, by (5.15),

$$
P_{k}\left(K_{n}-V_{-h(n)} K_{n} V_{h(n)}\right) x_{n} \rightarrow 0, \quad n \rightarrow \infty,
$$


for each $k \in \mathbb{N}$, so that $\left(K_{n}-V_{-h(n)} K_{n} V_{h(n)}\right) x_{n} \stackrel{s}{\rightarrow} 0$ by 2.6 . Thus $K_{n} x_{n}$ has a strictly convergent subsequence, so that, by Remark 3.23, $\sigma^{\mathrm{op}}(K)$ is uniformly Montel.

Conversely, suppose that $K$ is rich and $\sigma^{\mathrm{op}}(K)$ is uniformly Montel. Take an arbitrary sequence $h=(h(n))_{n=1}^{\infty} \subset \mathbb{Z}^{N}$ which tends to infinity and an arbitrary bounded sequence $\left(x_{n}\right) \subset Y$. Since $K$ is rich, $(h(n))$ and $\left(x_{n}\right)$ have subsequences, denoted again by $(h(n))$ and $\left(x_{n}\right)$, such that $V_{-h(n)} K V_{h(n)} \stackrel{\mathcal{P}}{\rightarrow} K_{h} \in \sigma^{\mathrm{op}}(K)$. Thus

$$
P_{k}\left(V_{-h(n)} K V_{h(n)}-K_{h}\right) x_{n} \rightarrow 0, \quad n \rightarrow \infty,
$$

for each $k \in \mathbb{N}$, so that $\left(V_{-h(n)} K V_{h(n)}-K_{h}\right) x_{n} \stackrel{s}{\rightarrow} 0$. On the other hand, $K_{h} x_{n}$ has a strictly convergent subsequence since $K_{h}$ is Montel. Thus $V_{-h(n)} K V_{h(n)} x_{n}$ has a strictly convergent subsequence.

REMARK 5.18 Note that, clearly, $\left(V_{-k} K V_{k}\right)_{k \in \mathbb{Z}^{N}}$ is asymptotically Montel iff $\left(V_{-k} K\right)_{k \in \mathbb{Z}^{N}}$ is asymptotically Montel since $\mathcal{V}=\left\{V_{k}\right\}_{k \in \mathbb{Z}^{N}} \subset i$ so $(Y)$.

An extension of Theorem 5.16 can be derived by applying Theorem 5.9 to

$$
\mathcal{K}:=\sigma^{\mathrm{op}}(K) \cup \mathcal{T}(K)
$$

with $\mathcal{T}(K)$ defined by (5.11), so that $I-\mathcal{K}=\sigma^{\mathrm{op}}(A) \cup \mathcal{T}(A)$. Properties (ii) and (iii) of Theorem 5.9 can be checked in a similar way as before. Property (i) of Theorem 5.9, that $\mathcal{K}$ is uniformly Montel on $(Y, s)$, is equivalently characterised by any of the properties (i)-(iii) of Lemma 6.23 below, which are equivalent even for arbitrary $K \in L(Y)$. Note that, for a rich operator $K$, by Lemma 5.17, any of these properties is moreover equivalent to $\sigma^{\mathrm{op}}(K)$ being uniformly Montel on $(Y, s)$ and $K \in M(Y)$. Then we get the following slightly enhanced version of the first part of Theorem 5.16, which in addition allows to conclude from $A$ being injective to the closedness of the range of $A$.

Theorem 5.19. Suppose $Y=\hat{Y}, A=I-K \in S(Y)$ is rich, $\mathcal{V}$ is sufficient, $K$ is subject to any of (i)-(iii) of Lemma 6.23, and $A$ as well as all its limit operators are injective. Then $A$ is bounded below and $\sigma^{\mathrm{op}}(A)$ is uniformly bounded below.

Note that Theorems 5.16 and 5.19 are applications of Theorem 5.9 which was just a special case of Theorem 5.8 . We will now apply Theorem 5.8 directly.

Theorem 5.20. Suppose that $Y=\hat{Y}, \mathcal{V}$ is sufficient, $A=I-K \in L(Y)$, $A_{n}=I-K_{n} \in L(Y)$ for $n \in \mathbb{N}$ and that:

(a) $A_{n} \stackrel{s}{\rightarrow} A$;

(b) $A_{n}$ bounded below $\Rightarrow A_{n}$ surjective, for each $n \in \mathbb{N}$;

(c) $\cup_{n \in \mathbb{N}} \mathcal{T}\left(K_{n}\right)=\left\{V_{-k} K_{n} V_{k}: k \in \mathbb{Z}^{N}, n \in \mathbb{N}\right\}$ is uniformly Montel on $(Y, s)$;

(d) there exists a set $\mathcal{B} \subset L(Y)$, such that, for every sequence $(k(m)) \subset \mathbb{Z}^{N}$ and increasing sequence $(n(m)) \subset \mathbb{N}$, there exist subsequences, denoted again by $(k(m))$ and $(n(m))$, and $B \in \mathcal{B}$ such that

$$
V_{-k(m)} A_{n(m)} V_{k(m)} \stackrel{s}{\rightarrow} B \in \mathcal{B} \quad \text { as } \quad m \rightarrow \infty ;
$$

(e) every $B \in \mathcal{B}$ is injective.

Then $A$ is invertible and, for some $n_{0} \in \mathbb{N}, A_{n}$ is invertible for all $n \geq n_{0}$, and

$$
\left\|A^{-1}\right\| \leq \sup _{n \geq n_{0}}\left\|A_{n}^{-1}\right\|<\infty
$$


Proof. Let $\mathcal{K}_{n}:=\mathcal{T}\left(K_{n}\right), n \in \mathbb{N}$, and set $\mathcal{K}:=I-\mathcal{B}, S:=\mathcal{V}$. Then (c) and (d) imply that conditions (i)-(iv) of Theorem 5.8 are satisfied, and (a) and (b) imply that the condition in Theorem $5.8 \mathrm{~b}$ ) is satisfied. Thus, applying Theorem 5.8 the result follows.

Later on, in Chapter 6, this result will be used to derive Theorem 6.37 on the invertibility of norm-rich/almost periodic band operators. As described at the end of Section 6.3, this result has also been used to prove Propositions 3.4 and 3.8 in 17.

REMARK 5.21 Note that condition (a) in the above theorem implies that $A \in S(Y)$ by Lemma 4.5. Moreover, from condition (d) with $k(m)=0$ for all $m \in \mathbb{N}$ and condition (a) again we get that $A \in \mathcal{B}$. Since $A \in S(Y)$ it holds that $\sigma^{\mathrm{op}}(A) \subset S(Y)$ (see discussion at the end of Section 5.3 ; ; if also $A_{n} \stackrel{\mathcal{P}}{\rightarrow} A$ then condition (d) also implies that $\sigma^{\mathrm{op}}(A) \subset \mathcal{B}$. To see this last claim, suppose that $\tilde{A} \in \sigma^{\mathrm{op}}(A)$. Then there exists $(k(m)) \subset \mathbb{Z}^{N}$ such that $V_{-k(m)} A V_{k(m)} \stackrel{\mathcal{P}}{\rightarrow} \tilde{A}$ which implies, in particular, that $\left\|P_{j}\left(\tilde{A}-V_{-k(m)} A V_{k(m)}\right)\right\| \rightarrow 0$ as $m \rightarrow \infty$, for every $j$. Choose the sequence $(l(m)) \subset \mathbb{N}$ such that $P_{n} V_{-k(m)} P_{l(m)}=P_{n} V_{-k(m)}$, for $n=1, \ldots, m$, which is possible by $(5.10)$. Then

$$
\left\|P_{j}\left(\tilde{A}-V_{-k(m)} A V_{k(m)}\right)\right\|=\left\|P_{j}\left(\tilde{A}-V_{-k(m)} P_{l(m)} A V_{k(m)}\right)\right\|
$$

for all $j$ and all $m>j$. Since $A_{n} \stackrel{\mathcal{P}}{\rightarrow} A$, for every $m$ we can choose $n(m)$ such that $\left\|P_{l(m)}\left(A_{n(m)}-A\right)\right\|<m^{-1}$. Then

$$
\left\|P_{j}\left(\tilde{A}-V_{-k(m)} A_{n(m)} V_{k(m)}\right)\right\|=\left\|P_{j}\left(\tilde{A}-V_{-k(m)} P_{l(m)} A_{n(m)} V_{k(m)}\right)\right\| \rightarrow 0
$$

as $m \rightarrow \infty$, for every $j$, so that, by Lemma 4.7 . $V_{-k(m)} A_{n(m)} V_{k(m)} \stackrel{s}{\rightarrow} \tilde{A}$, and so $\tilde{A} \in \mathcal{B}$.

In the case that $A=I-K$ with $K \in S(Y) \cap M(Y)$, one particular choice of $A_{n}$ which satisfies (a) and (b) is

$$
A_{n}=I-K P_{n}
$$

For, by Lemma 3.21 $K P_{n} \in K S(Y)$ for every $n$ so that, by a version of Riesz Fredholm theory for TVS's (see e.g. [77), assumption (b) holds. Further, by Corollary 3.5 , for every $m$,

$$
\left\|P_{m}\left(A_{n}-A\right)\right\|=\left\|P_{m} K Q_{n}\right\| \rightarrow 0, \quad n \rightarrow \infty
$$

so that, by Lemma 4.7, $A_{n} \stackrel{s}{\rightarrow} A$. Further, if $\mathcal{T}(K)=\left\{V_{-k} K V_{k}: k \in \mathbb{Z}^{N}\right\}$ is uniformly Montel on $(Y, s)$, then so is $\left\{V_{-k} K: k \in \mathbb{Z}^{N}\right\}$ and hence also $\left\{V_{-k} K P_{n} V_{k}\right.$ : $\left.k \in \mathbb{Z}^{N}, n \in \mathbb{N}\right\}=\cup_{n \in \mathbb{N}} \mathcal{T}\left(K P_{n}\right)$. Thus Theorem 5.20 has the following corollary.

Corollary 5.22. Suppose that $Y=\hat{Y}, \mathcal{V}$ is sufficient, $A=I-K$ with $K \in S(Y)$, and set $A_{n}=I-K P_{n}$ for $n \in \mathbb{N}$. Suppose that $\mathcal{T}(K)$ is uniformly Montel on $(Y, s)$, and that there exists $\mathcal{B} \subset L(Y)$ such that every $B \in \mathcal{B}$ is injective and for every sequence $(k(m)) \subset \mathbb{Z}^{N}$ and increasing sequence $(n(m)) \subset \mathbb{N}$, there exist subsequences, denoted again by $k(m)$ and $n(m)$, and $B \in \mathcal{B}$, such that

$$
I-V_{-k(m)} K P_{n(m)} V_{k(m)} \stackrel{s}{\rightarrow} B \in \mathcal{B} .
$$

Then $A$ is invertible and, for some $n_{0} \in \mathbb{N}, A_{n}$ is invertible for all $n \geq n_{0}$, and

$$
\left\|A^{-1}\right\| \leq \sup _{n \geq n_{0}}\left\|A_{n}^{-1}\right\|<\infty
$$


Remark 5.23 We note, by Remark 5.21, that, necessarily, $A \in \mathcal{B}$ and $\sigma^{\mathrm{op}}(A) \subset$ $\mathcal{B}$.

More concrete statements than Theorems 5.16,5.19 and 5.20 can be given when we pass to a more concrete class of spaces $Y$. This is what we do in Chapter 6 . 


\section{CHAPTER 6}

\section{Operators on $\ell^{p}\left(\mathbb{Z}^{N}, U\right)$}

In this chapter we focus on the case, introduced already briefly in Example 4.3 . when $Y=\ell^{p}\left(\mathbb{Z}^{N}, U\right)$, where $1 \leq p \leq \infty, N \in \mathbb{N}$ and $U$ is an arbitrary complex Banach space. The elements of $Y$ are of the form $x=(x(m))_{m \in \mathbb{Z}^{N}}$ with $x(m) \in U$ for every $m=\left(m_{1}, \ldots, m_{N}\right) \in \mathbb{Z}^{N}$. We equip $Y$ with the usual $\ell^{p}$ norm of the scalar sequence $\left(\|x(m)\|_{U}\right)$. We also consider the case when $Y=c_{0}\left(\mathbb{Z}^{N}, U\right)$, the Banach subspace of $\ell^{\infty}\left(\mathbb{Z}^{N}, U\right)$ consisting of the elements that vanish at infinity, i.e. $\|x(m)\|_{U} \rightarrow 0$ as $m \rightarrow \infty$.

Since the parameter $N \in \mathbb{N}$ is of no big importance in almost all of what follows, we will use the abbreviations $Y^{0}(U):=c_{0}\left(\mathbb{Z}^{N}, U\right)$ and $Y^{p}(U):=\ell^{p}\left(\mathbb{Z}^{N}, U\right)$ for $1 \leq p \leq \infty$. If there is no danger of confusion about what $U$ is, we will even write $Y^{0}$ and $Y^{p}$. Some of our following statements hold for all the spaces under consideration. In this case we will simply write $Y$, which then can be replaced by any of $Y^{0}$ and $Y^{p}$ with $1 \leq p \leq \infty$.

In terms of dual spaces, we have $\left(Y^{0}(U)\right)^{*} \cong Y^{1}\left(U^{*}\right),\left(Y^{1}(U)\right)^{*} \cong Y^{\infty}\left(U^{*}\right)$, and $\left(Y^{p}(U)\right)^{*} \cong Y^{q}\left(U^{*}\right)$ for $1<p<\infty$ and $1 / p+1 / q=1$ (see e.g. [81]). To give two prominent examples, the space $Y^{p}(\mathbb{C})$ is the usual $\ell^{p}$ or $c_{0}$ space of complex-valued sequences over $\mathbb{Z}^{N}$, and the space $Y^{p}\left(L^{p}\left([0,1]^{N}\right)\right)$ is isometrically isomorphic to $L^{p}\left(\mathbb{R}^{N}\right)$ for $1 \leq p \leq \infty$; see Chapter 8 or [43, 74, 51] for a more detailed discussion of this isomorphism.

For $m \in \mathbb{Z}^{N}$, we define $|m|:=\max \left(\left|m_{1}\right|, \ldots,\left|m_{N}\right|\right)$ and put

$$
P_{n} x(m)= \begin{cases}x(m), & |m| \leq n, \\ 0, & |m|>n,\end{cases}
$$

for every $x \in Y$ and $n \in \mathbb{N}_{0}$. As observed already in Example $4.3 \mathcal{P}=\left(P_{n}\right)$ satisfies conditions (i) and (ii) of Chapter 2 with $N(m)=m$, so that $P_{n}$ is a projection operator for each $n$. In this case $\left\|Q_{n}\right\|=1$ for all $n$. For $p \in\{0\} \cup[1, \infty)$, we have $\left(Y^{p}\right)_{0}=Y^{p}$, while $\left(Y^{\infty}\right)_{0}=Y^{0}$. Moreover, for $p \in[1, \infty]$, we have $\widehat{Y^{p}}=Y^{p}$ (cf. Example 2.8, whereas $\widehat{Y^{0}}=Y^{\infty}$.

In the setting of this chapter we have the following refinement of Lemma 3.13 .

LEMma 6.1. The following two statements hold.

(i) If $p \in\{0\} \cup(1, \infty)$ then $K\left(Y^{p}\right) \subset K\left(Y^{p}, \mathcal{P}\right)$.

(ii) If $U$ is finite-dimensional then $K(Y, \mathcal{P}) \subset K(Y)$.

Proof. (i) Let $p \in\{0\} \cup(1, \infty)$. Then $P_{n} \rightarrow I$ as well as $P_{n}^{*} \rightarrow I^{*}$, on $Y^{p}$ and $\left(Y^{p}\right)^{*}$, respectively, as $n \rightarrow \infty$, i.e. $\mathcal{P}$ is perfect. Thus $K\left(Y^{p}\right) \subset K\left(Y^{p}, \mathcal{P}\right)$ by Lemma 3.15 . 
(ii) If $\operatorname{dim} U<\infty$ then $P_{n} \in K(Y)$ for all $n$, and, by Lemma 3.13, we get $K(Y, \mathcal{P})=L(Y, \mathcal{P}) \cap K(Y) \subset K(Y)$.

REMARK 6.2 From Lemma 6.1 and (5.1) we conclude another result, in addition to Theorem 5.4 and Corollary 5.5, which relates Fredholmness to invertibility at infinity in the setting of this chapter: For an arbitrary operator $A \in L\left(Y^{p}(U)\right)$, Fredholmness implies invertibility at infinity if $p \in\{0\} \cup(1, \infty)$, and invertibility at infinity implies Fredholmness if $\operatorname{dim} U<\infty$.

If we moreover put, for $x \in Y$,

$$
V_{k} x(m):=x(m-k), \quad m \in \mathbb{Z}^{N}
$$

for every $k \in \mathbb{Z}^{N}$, then the family $\mathcal{V}=\left\{V_{k}\right\}_{k \in \mathbb{Z}^{N}}$ consists of isometric isomorphisms on $Y$ and satisfies conditions $(5.8)-(5.10)$ so that it is compatible with $\mathcal{P}$.

In the case $p=\infty$ (but not if $1 \leq p<\infty$ ) the family $\mathcal{V}=\left\{V_{k}\right\}_{k \in \mathbb{Z}^{N}}$ is sufficient (see Example 5.7). Thus each of Theorems 5.16, 5.19, 5.20 and Corollary 5.22 can be applied in the case $Y=Y^{\infty}$. Note also that for $Y^{\infty}$ Assumption A of Chapter 4 holds with $Q=Q_{m}$ and $n=m+1$ so that Corollary 4.14 applies and that therefore the convergence $\stackrel{s}{\rightarrow}$ can be replaced by the formally weaker notion

$\stackrel{S}{\rightarrow}$ of convergence in the strong operator topology on $\left(Y^{\infty}, s\right)$, defined by 4.7). Applying Theorem 5.16, for example, we have the following result in which we say that a subset $A \subset \mathcal{K} \subset L\left(Y^{\infty}\right)$ is $S$-dense in $\mathcal{K}$ if, for every $K \in \mathcal{K}$, there is a sequence $\left(K_{n}\right) \subset A$ with $K_{n} \stackrel{S}{\rightarrow} K$.

TheOREM 6.3. Suppose $A=I-K \in S\left(Y^{\infty}\right)$ is rich, $\sigma^{\mathrm{op}}(K)$ is uniformly Montel on $\left(Y^{\infty}, s\right)$, and all the limit operators of $A$ are injective. Then $\sigma^{\mathrm{op}}(A)$ is uniformly bounded below. If, moreover, there is an $S$-dense subset of surjective operators in $\sigma^{\mathrm{op}}(A)$ then all elements of $\sigma^{\mathrm{op}}(A)$ are invertible and their inverses are uniformly bounded.

REMARK 6.4 We have seen in Lemma 5.17 that $\sigma^{\mathrm{op}}(K)$ is uniformly Montel on $\left(Y^{\infty}, s\right)$ iff the sequence $\left(V_{-k} K V_{k}\right)_{k \in \mathbb{Z}^{N}}$ is asymptotically Montel. If the Banach space $U$ is of finite dimension, then the condition that $\sigma^{\mathrm{op}}(K)$ be uniformly Montel on $\left(Y^{\infty}, s\right)$ is even redundant. For $U$ finite-dimensional implies that $P_{n} \in K\left(Y^{\infty}\right)$ for all $n$, so that $\sigma^{\mathrm{op}}(K)$ is uniformly Montel by Corollary 3.25 and Theorem 5.12 (i). $\square$

\subsection{Periodic and Almost Periodic Operators}

With regard to Theorem 6.3 we remark that we know of no examples where the requirement for an $S$-dense subset of surjective operators is not redundant. Precisely, in all the examples we have studied existence of an $S$-dense subset of $\sigma^{\mathrm{op}}(A)$ of surjective operators can be deduced from injectivity of all the elements of $\sigma^{\mathrm{op}}(A)$. To illustrate Theorem 6.3 we present next an important example of this type in which even invertibility of $A$ (on $Y^{\infty}$ ) follows from injectivity of all limit operators of $A$. We will present further examples in sections 6.3 and 6.4 .

To introduce this example we require the following definitions. Recall from Theorem 5.12 (v) that $A \in L(Y)$ is rich iff the set $\mathcal{T}(A)$ of all translates of $A$ (recall (5.11) ) is relatively $\mathcal{P}$-sequentially compact. Now call $A \in L(Y)$ norm-rich or almost periodic if the set $\mathcal{T}(A)$ is relatively compact in the norm topology on 
$L(Y)$ (this is precisely the definition of an almost periodic operator in Kurbatov 42]). Call $A \in L(Y)$ absolutely rich or periodic if every sequence in $\mathcal{T}(A)$ has a constant subsequence, i.e. iff $\mathcal{T}(A)$ is a finite set. It is easy to establish the following characterisation.

Lemma 6.5. An operator $A \in L(Y)$ is absolutely rich/periodic iff there exist $m_{1}, \ldots, m_{N} \in \mathbb{N}$ such that

$$
V A=A V \quad \text { for all } \quad V \in \tilde{\mathcal{V}}_{A}:=\left\{V_{m_{j} e^{(j)}}\right\}_{j=1}^{N}
$$

with $e^{(1)}, \ldots, e^{(N)}$ denoting the standard unit vectors in $\mathbb{R}^{N}$, i.e. $e^{(j)}(i)=1$ if $i=j$ and $=0$ otherwise.

EXAmPLE 6.6 For $b=(b(m))_{m \in \mathbb{Z}^{N}} \in \ell^{\infty}\left(\mathbb{Z}^{N}, L(U)\right)=Y^{\infty}(L(U))$ define the multiplication operator $M_{b} \in L(Y)$, as in Example 4.3. by equation (4.4). Then, for $k \in \mathbb{Z}^{N}$,

$$
V_{-k} M_{b} V_{k}=M_{V_{-k} b} \quad \text { and } \quad\left\|M_{b}\right\|=\|b\| .
$$

From these identities and (4.5), it is immediate that $M_{b}$ is rich iff the set

$$
\left\{V_{k} b\right\}_{k \in \mathbb{Z}^{N}}
$$

is relatively sequentially compact in the strict topology on $Y^{\infty}(L(U))$. It can be shown moreover [74, Theorem 2.1.16] that this is the case iff the set $\{b(m): m \in$ $\left.\mathbb{Z}^{N}\right\}$ is relatively compact in $L(U)$. Also, it is clear from 6.2 that $M_{b}$ is norm rich iff the set 6.3$)$ is relatively compact in the norm topology on $Y^{\infty}(L(U))$; it is usual to say (cf. Example 2.10 and [51, Definition 3.58]) that $b$ is almost periodic if this condition on $b$ holds. The set of all almost periodic sequences $b \in Y^{\infty}(Z)$ with a Banach space $Z$ shall be denoted by $Y_{\mathrm{AP}}^{\infty}(Z)$.

Similarly, $M_{b}$ is absolutely rich/periodic iff every sequence in 6.3 has a constant subsequence, i.e. iff 6.3 is finite. By Lemma 6.5 this is equivalent to the requirement that there exist $m_{1}, \ldots, m_{N} \in \mathbb{N}$ such that

$$
b\left(k+m_{j} e^{(j)}\right)=b(k), \quad k \in \mathbb{Z}^{N}, j=1, \ldots, N,
$$

i.e. to the requirement that $b(k)$ is periodic as a function of each of the components of $k \in \mathbb{Z}^{N}$.

Suppose that $A \in L\left(Y^{\infty}\right)$ is absolutely rich/periodic. For $n \in \mathbb{N}$, let

$$
Y_{n}^{\infty}=Y_{n}^{\infty}(U):=\left\{x \in Y^{\infty}(U): V^{n} x=x \text { for all } V \in \tilde{\mathcal{V}}_{A}\right\}
$$

with $\tilde{\mathcal{V}}_{A}$ as defined in Lemma 6.5. Then $Y_{n}^{\infty}$ is a closed subspace of $Y^{\infty}$ consisting of periodic elements; $x \in Y_{n}^{\infty}$ iff

$$
x\left(k+n m_{j} e^{(j)}\right)=x(k), \quad k \in \mathbb{Z}^{N}, j=1, \ldots, N,
$$

where the integers $m_{1}, \ldots, m_{N}$ are as in the definition of $\tilde{\mathcal{V}}_{A}$ in Lemma 6.5. Clearly, $x \in Y_{n}^{\infty}$ is determined by its components in the box

$$
C_{n}:=\left\{i=\left(i_{1}, \ldots, i_{N}\right) \in \mathbb{Z}^{N}:-n \frac{m_{j}}{2}<i_{j} \leq n \frac{m_{j}}{2}, j=1, \ldots, N\right\} .
$$

Define the projection operator $\tilde{P}_{n}: Y^{\infty} \rightarrow Y_{n}^{\infty}$ by the requirement that

$$
\tilde{P}_{n} x(k)=x(k) \quad \text { for all } \quad k \in C_{n} .
$$


Then, clearly, for each $n, \tilde{P}_{n} Q_{j}=0$ for all sufficiently large $j$, so that $\tilde{P}_{n} \in S N\left(Y^{\infty}\right)$ by Lemma 3.3 . (Note however that $\tilde{P}_{n} \notin L\left(Y^{\infty}, \mathcal{P}\right)$.)

The last part of the following result and its proof can be seen as a generalisation of Theorem 2.10 in 20 .

TheOREM 6.7. If $A \in L\left(Y^{\infty}\right)$ is absolutely rich/periodic then $A\left(Y_{n}^{\infty}\right) \subset Y_{n}^{\infty}$ for each $n$, and

$$
\sigma^{\mathrm{op}}(A)=\left\{V_{-i} A V_{i}: i \in \mathbb{Z}^{N}\right\}=\left\{V_{-i} A V_{i}: i \in C_{1}\right\} .
$$

If also $A=I+K$ with $K \in S\left(Y^{\infty}\right) \cap M\left(Y^{\infty}\right)$ and $A$ is injective then $A$ is invertible.

Proof. If $x \in Y_{n}^{\infty}$ then $A x \in Y_{n}^{\infty}$ since $V^{n}(A x)=A V^{n} x=A x$ for every $V \in \tilde{\mathcal{V}}_{A}$. From the definitions and Lemma 6.5 it is clear that $(6.6)$ holds. Suppose now that $K \in S\left(Y^{\infty}\right) \cap M\left(Y^{\infty}\right)$ and that $A=I+K$ is injective. First we show that, for every $n, I+K \tilde{P}_{n}$ is invertible. To see injectivity, suppose $x \in Y^{\infty}$ and $\left(I+K \tilde{P}_{n}\right) x=0$. Then $x=-K \tilde{P}_{n} x \in Y_{n}^{\infty}$ since $\tilde{P}_{n} x \in Y_{n}^{\infty}$ and $K=A-I$ is absolutely rich. Now $x \in Y_{n}^{\infty}$ implies $\tilde{P}_{n} x=x$ and therefore $0=\left(I+K \tilde{P}_{n}\right) x=(I+K) x=A x$, i.e. $x=0$ by injectivity of $A$. Now surjectivity of $I+K \tilde{P}_{n}$ follows from its injectivity by the Riesz theory for compact operators in topological vector spaces [77] since $K \tilde{P}_{n} \in K S\left(Y^{\infty}\right)$ by Lemma 3.31 .

Next, note that from (6.6) it follows that $A \in \sigma^{\mathrm{op}}(A)$ and that, since $A$ is injective, all the limit operators of $A$ are injective. Further, by (6.6) and Remark 6.4. it follows that $\sigma^{\mathrm{op}}(K)$ is uniformly Montel since $K \in M\left(Y^{\infty}\right)$. Applying Theorem 6.3 we see that the limit operators of $A$ are uniformly bounded below, in particular that $A$ is bounded below.

To see finally that $A$ is surjective let $y \in Y^{\infty}$ and set $y_{n}=\tilde{P}_{n} y \in Y_{n}^{\infty}$ and $x_{n}=\left(I+K \tilde{P}_{n}\right)^{-1} y_{n}$ so that $x_{n}+K \tilde{P}_{n} x_{n}=y_{n}$ which implies (as seen above) that $x_{n} \in Y_{n}^{\infty}$, that $\tilde{P}_{n} x_{n}=x_{n}$, and hence that $A x_{n}=x_{n}+K x_{n}=y_{n}$. Since $\left(y_{n}\right)$ is bounded and $A$ is bounded below, also $\left(x_{n}\right)$ is bounded. Since $K \in M\left(Y^{\infty}\right)$ it follows that there exists an $x \in Y^{\infty}$ and a subsequence of $\left(x_{n}\right)$, denoted again by $\left(x_{n}\right)$, such that $K x_{n} \stackrel{s}{\rightarrow} y-x$, so that $x_{n}=y_{n}-K x_{n} \stackrel{s}{\rightarrow} y-(y-x)=x$. As $K \in S\left(Y^{\infty}\right)$ this implies $K x_{n} \stackrel{s}{\rightarrow} K x$ so that $A x=x+K x=y$.

The above result has the following obvious corollary (phrased in the spirit of Theorem 6.3. which was the starting point of this discussion).

Corollary 6.8. If $A=I+K \in S\left(Y^{\infty}\right)$ is absolutely rich/periodic and $K \in$ $M\left(Y^{\infty}\right)$ and if all limit operators of $A$ are injective, then all limit operators of $A$ are invertible (with uniformly bounded inverses).

Further down, in Theorem 6.38 we will show that, in the case when $A$ is also band-dominated (as defined in 86.3 ), this corollary holds more generally with 'absolutely rich' replaced by 'norm-rich'; indeed in the one-dimensional case $N=1$ we will show in Theorem 6.31 that this corollary holds even with 'absolutely rich' replaced by 'rich'. We conclude the current section by a collection of results for the general setting of all norm-rich/almost periodic operators $A \in L(Y)$; a set that shall be denoted by $L^{\mathrm{n} \$}(Y)$ for brevity. Our first result follows from a slightly more general result which is Theorem 6.5.2 in Kurbatov [43. We include a proof here for the convenience of the reader. 
Lemma 6.9. $L^{\mathrm{n} \$}(Y)$ is an inverse closed Banach subalgebra of $L(Y)$.

Proof. Let $A, B \in L^{\mathrm{n} \$}(Y)$ and take an arbitrary sequence $h=(h(1), h(2), \ldots) \subset \mathbb{Z}^{N}$. Pick a subsequence $g$ of $h$ such that both $V_{-g(n)} A V_{g(n)}$ and $V_{-g(n)} B V_{g(n)}$ converge in norm. Then, clearly, also $V_{-g(n)}(A+B) V_{g(n)}$ and

$$
V_{-g(n)}(A B) V_{g(n)}=\left(V_{-g(n)} A V_{g(n)}\right)\left(V_{-g(n)} B V_{g(n)}\right)
$$

converge in norm. To see that $L^{\mathrm{n} \$}(Y)$ is closed in the operator norm take $A_{1}, A_{2}, \ldots \in$ $L^{\mathrm{n} \$}(Y)$ with $A_{k} \rightrightarrows A \in L(Y)$ and an arbitrary sequence $h=(h(n))_{n=1}^{\infty} \subset \mathbb{Z}^{N}$. Pick subsequences $\cdots \subset h^{(2)} \subset h^{(1)} \subset h$ such that, for every $k \in \mathbb{N}$,

$$
\left\|V_{-h^{(k)}(m)} A_{k} V_{h^{(k)}(m)}-V_{-h^{(k)}(n)} A_{k} V_{h^{(k)}(n)}\right\|<1 / k, \quad m, n>k,
$$

and put $g(n):=h^{(n)}(n)$ for all $n \in \mathbb{N}$. Then, for all $k \in \mathbb{N}$ and all $m, n>k$, noting that $g(n)=h^{(k)}\left(n^{\prime}\right)$ for some $n^{\prime} \geq n>k$,

$$
\begin{aligned}
\left\|V_{-g(m)} A V_{g(m)}-V_{-g(n)} A V_{g(n)}\right\| \leq & \left\|V_{-g(m)} A_{k} V_{g(m)}-V_{-g(n)} A_{k} V_{g(n)}\right\| \\
& +2\left\|A_{k}-A\right\| \\
\leq & 1 / k+2\left\|A_{k}-A\right\| \rightarrow 0
\end{aligned}
$$

as $k \rightarrow \infty$. This shows that the sequence $\left(V_{-g(n)} A V_{g(n)}\right)$ is Cauchy and therefore convergent in $L(Y)$. Since $g \subset h$, we get that $A \in L^{\mathrm{n} \$}(Y)$.

To see the inverse closedness suppose $A \in L^{\mathrm{n} \$}(Y)$ is invertible in $L(Y)$ and take an arbitrary sequence $h=(h(1), h(2), \ldots) \subset \mathbb{Z}^{N}$. Since $A \in L^{\mathrm{n} \$}(Y)$, there is a subsequence $g$ of $h$ such that $A_{n}:=V_{-g(n)} A V_{g(n)} \rightrightarrows B$ for some $B \in L(Y)$. Since $\left\|A_{n}^{-1}\right\|=\left\|V_{-g(n)} A^{-1} V_{g(n)}\right\|=\left\|A^{-1}\right\|$ is bounded independently of $n$, it follows from a basic result on Banach algebras (see, e.g. Lemma 1.3 of [51]) that $B$ is invertible and

$$
A_{n}^{-1}=V_{-g(n)} A^{-1} V_{g(n)} \rightrightarrows B^{-1},
$$

showing that $A^{-1} \in L^{\mathrm{n} \$}(Y)$

Theorem 6.10. For $A \in L^{\mathrm{n} \$}(Y)$, the following holds.

(i) If, for some sequence $h=(h(1), h(2), \ldots) \subset \mathbb{Z}^{N}$ and $B \in L(Y)$,

$$
V_{-h(n)} A V_{h(n)} \stackrel{\mathcal{P}}{\rightarrow} B \quad \text { holds, then } \quad V_{-h(n)} A V_{h(n)} \rightrightarrows B .
$$

(ii) $A \in \sigma^{\mathrm{op}}(A)$ (i.e. $A$ is self-similar).

(iii) $\sigma^{\mathrm{op}}(A)=\operatorname{clos}_{L(Y)} \mathcal{T}(A)$ is a compact subset of $L^{\mathrm{n} \$}(Y)$.

(iv) $A$ is invertible iff any one of its limit operators is invertible.

(v) $\nu(A)=\nu(B)$ for all $B \in \sigma^{\mathrm{op}}(A)$, so that $A$ is bounded below iff $\sigma^{\mathrm{op}}(A)$ is uniformly bounded below.

(vi) If $x$ is almost periodic, then $A x$ is almost periodic.

(vii) If $A$ is invertible on $Y^{\infty}$, then it is invertible on $Y_{\mathrm{AP}}^{\infty}$.

Proof. (i) Since $A \in L^{\mathrm{n}}(Y)$, every subsequence of $V_{-h(n)} A V_{h(n)}(\stackrel{\mathcal{P}}{\rightarrow} B)$ has a norm-convergent subsequence the limit of which must be $B$. But this proves norm convergence of the whole sequence.

(ii) Let $h(n)=\left(n^{2}, 0, \ldots, 0\right) \in \mathbb{Z}^{N}$ for every $n \in \mathbb{N}$. Since $A \in L^{\mathrm{n} \$}(Y)$, there is a subsequence $g$ of $h$ such that $V_{-g(n)} A V_{g(n)}$ converges. But then

$$
\left\|V_{-(g(n+1)-g(n))} A V_{g(n+1)-g(n)}-A\right\|=\left\|V_{-g(n+1)} A V_{g(n+1)}-V_{-g(n)} A V_{g(n)}\right\| \rightarrow 0
$$


as $n \rightarrow \infty$, showing that $A=A_{f} \in \sigma^{\mathrm{op}}(A)$ with $f(n)=g(n+1)-g(n) \rightarrow \infty$.

(iii) The inclusion $\sigma^{\mathrm{op}}(A) \subset \cos _{L(Y)} \mathcal{T}(A)$ follows from (i). The reverse inclusion follows from (ii), from Theorem 5.12 (ii) and the closedness of $\sigma^{\mathrm{op}}(A)$ (see Theorem 5.12 (iii) above or [51, Corollary 3.96]). The compactness of $\operatorname{clos}_{L(Y)} \mathcal{T}(A)$ follows from the relative compactness of $\mathcal{T}(A)$ in $L(Y)$. By Lemma 6.9, every operator in $\operatorname{clos}_{L(Y)} \mathcal{I}(A)$ is norm-rich/almost periodic.

(iv) Take an arbitrary limit operator $A_{h}$ of $A$ and let $h=(h(1), h(2), \ldots) \subset \mathbb{Z}^{N}$ be such that $A_{n}:=V_{-h(n)} A V_{h(n)} \stackrel{\mathcal{P}}{\rightarrow} A_{h}$ holds. By (i) we have that $A_{n} \rightrightarrows A_{h}$. If $A_{h}$ is invertible, then so is $A_{n}$ for every large $n$, and therefore $A$ is invertible. Conversely, if $A$ is invertible, then $A_{h}$ is invertible by a basic result on Banach algebras (see e.g. [51, Lemma 1.3]) since $\left\|A_{n}^{-1}\right\|=\left\|A^{-1}\right\|$ is bounded.

(v) If $B \in \sigma^{\mathrm{op}}(A)$, then, by (i), we have that $V_{-h(n)} A V_{h(n)} \rightrightarrows B$ for some sequence $h(1), h(2), \ldots$ in $\mathbb{Z}^{N}$. By 5.6 this implies that $\nu\left(V_{-h(n)} A V_{h(n)}\right) \rightarrow \nu(B)$ as $n \rightarrow \infty$. On the other hand, since every $V_{h(n)}$ is an isometry, we have that

$$
\nu\left(V_{-h(n)} A V_{h(n)}\right)=\inf _{\|x\|=1}\left\|V_{-h(n)} A V_{h(n)} x\right\|=\inf _{\|y\|=1}\|A y\|=\nu(A)
$$

for every $n \in \mathbb{N}$, so that $\nu(A)=\nu\left(V_{-h(n)} A V_{h(n)}\right) \rightarrow \nu(B)$, i.e. $\nu(A)=\nu(B)$.

(vi) Let $h=(h(1), h(2), \ldots) \subset \mathbb{Z}^{N}$ be arbitrary. If $A \in L^{\mathrm{n} \$}\left(Y^{\infty}\right)$ and $x \in Y_{\mathrm{AP}}^{\infty}$ there is a subsequence $g$ of $h$ such that both $V_{g(n)} A V_{-g(n)}$ and $V_{g(n)} x$ converge in the norm of $L\left(Y^{\infty}\right)$ and $Y^{\infty}$, respectively. But then also

$$
V_{g(n)}(A x)=\left(V_{g(n)} A V_{-g(n)}\right)\left(V_{g(n)} x\right)
$$

converges in $Y^{\infty}$, which shows that $A x \in Y_{\mathrm{AP}}^{\infty}$.

(vii) If $A \in L^{\mathrm{n} \$}\left(Y^{\infty}\right)$ is invertible on $Y^{\infty}$, then, by Lemma 6.9, also $A^{-1} \in$ $L^{\mathrm{n} \$}\left(Y^{\infty}\right)$. Now (vi) shows that $x \in Y_{\mathrm{AP}}^{\infty}$ iff $A x \in Y_{\mathrm{AP}}^{\infty}$.

\subsection{Dual Space Arguments}

In the results we will present below dual space arguments will play a role, in particular in the cases $p=1$ and $p=\infty$. Temporarily set $Y=\ell^{\infty}\left(\mathbb{Z}^{N}, U\right)$ so that $Y_{0}=c_{0}\left(\mathbb{Z}^{N}, U\right)$, and set $Y_{1}=\ell^{1}\left(\mathbb{Z}^{N}, U^{*}\right)$, where $U^{*}$ is the dual space of $U$. Note that $Y_{1}=Y_{0}^{*}$. Further, $Y_{1}^{*}=Y$ if $U$ is reflexive, i.e. if $U=U^{* *}$. In the general case when $U$ is not reflexive we shall see that we can, in a natural way, embed $Y$ as a closed subspace of $Y_{1}^{*}$.

For $x \in Y, y \in Y_{1}$, define the bilinear form $(\cdot, \cdot)$ on $\left(Y, Y_{1}\right)$ by

$$
(x, y):=\sum_{j \in \mathbb{Z}^{N}} y_{j}\left(x_{j}\right), \quad \text { for } x=\left(x_{j}\right)_{j \in \mathbb{Z}^{N}} \in Y, y=\left(y_{j}\right)_{j \in \mathbb{Z}^{N}} \in Y_{1},
$$

and note that, equipped with $(\cdot, \cdot),\left(Y, Y_{1}\right)$ is a dual system in the sense e.g. of Jörgens [38. If $x \in Y_{0}$ and $y \in Y_{1}=Y_{0}^{*}$ then $(x, y)=y(x)$. A similar equation holds if $U$ has a predual space $U^{\triangleleft}$, i.e. if there exists a Banach space $U^{\triangleleft}$ such that $\left(U^{\triangleleft}\right)^{*}=U$. Then $Y$ is the dual space of $Y^{\triangleleft}$, where $Y^{\triangleleft}:=\ell^{1}\left(\mathbb{Z}^{N}, U^{\triangleleft}\right)$. Denote by $J_{U}$ the canonical embedding of $U^{\triangleleft}$ into its second dual $U^{*}$, given by $J_{U} u(v)=v(u)$, $u \in U^{\triangleleft}, v \in U$, and let $J^{\triangleleft}: \ell^{1}\left(\mathbb{Z}^{N}, U^{\triangleleft}\right) \rightarrow \ell^{1}\left(\mathbb{Z}^{N}, U^{*}\right)$ be the natural embedding $J^{\triangleleft} x=\left(J_{U} x_{j}\right)_{j \in \mathbb{Z}^{N}}$. Note that both $J_{U}$ and $J^{\triangleleft}$ are isometries. Then

$$
\left(x, J^{\triangleleft} y\right)=x(y), \quad x \in Y, y \in Y^{\triangleleft} .
$$


An important observation is that, if $\left(x_{n}\right) \subset Y, x \in Y$, and $y \in Y_{1}$, then

$$
x_{n} \stackrel{s}{\rightarrow} x \quad \Rightarrow \quad\left(x_{n}, y\right) \rightarrow(x, y) .
$$

For every $A \in L_{0}(Y)$ (recall the definition (3.6) of $L_{0}(Y)$ ) let $A_{0} \in L\left(Y_{0}\right)$ be defined by $A_{0}:=\left.A\right|_{Y_{0}}$. Then its adjoint $A_{0}^{*} \in L\left(Y_{0}^{*}\right)=L\left(Y_{1}\right)$. From Corollary 3.17 we recall that, in particular, $A \in L_{0}(Y)$ if $A \in L(Y, \mathcal{P}) \subset S(Y)$.

Lemma 6.11. If $A \in S(Y) \cap L_{0}(Y)$ then

$$
(A x, y)=\left(x, A_{0}^{*} y\right), \quad x \in Y, y \in Y_{1},
$$

i.e. $A$ is the transpose of $A_{0}^{*}$ with respect to the dual system $\left(Y, Y_{1}\right)$.

Proof. For $x \in Y_{0}, y \in Y_{0}^{*}=Y_{1}$,

$$
(A x, y)=\left(A_{0} x, y\right)=\left(x, A_{0}^{*} y\right) .
$$

Thus, for $x \in Y, y \in Y_{1}$,

$$
\left(A P_{n} x, y\right)=\left(P_{n} x, A_{0}^{*} y\right) .
$$

Taking the limit $n \rightarrow \infty$, in view of 6.10$)$ and since $A \in S(Y)$, the result follows.

It follows from Lemma 6.1(i) that if $A_{0} \in L\left(Y_{0}\right)$ is Fredholm then it is invertible at infinity (cf. Remark 6.2). We shall see that under certain conditions the same implication holds for $A \in L(Y)$. Our tool to establish this will be to relate Fredholmness of $A$ to that of $A_{0}$. To this end a useful tool is to embed $Y$ as a closed subspace of $Y_{0}^{* *}=\ell^{\infty}\left(\mathbb{Z}^{N}, U^{* *}\right)$. Precisely, define $J: Y \rightarrow Y_{0}^{* *}$ by

$$
J x(y)=(x, y), \quad x \in Y, y \in Y_{0}^{*}=Y_{1} .
$$

It is easy to check that $J$ is an isometry, so that $Y$ is isometrically isomorphic to $\check{Y}:=J(Y) \subset Y_{0}^{* *}$. For $A \in L(Y)$ define $\check{A} \in L(\check{Y})$ by $\check{A}:=J A J^{-1}$.

Lemma 6.12. If $A \in S(Y) \cap L_{0}(Y)$, then $A_{0}^{* *}(\check{Y}) \subset \check{Y}$ and $\check{A}=\left.A_{0}^{* *}\right|_{\check{Y}}$, so that

$$
\alpha\left(A_{0}\right) \leq \alpha(A)=\alpha(\check{A}) \leq \alpha\left(A_{0}^{* *}\right) .
$$

Proof. For $x \in \check{Y}, y \in Y_{1}$, with $z:=J^{-1} x \in Y$,

$$
\check{A} x(y)=(J(A z))(y)=(A z, y)=\left(z, A_{0}^{*} y\right),
$$

by Lemma 6.11, and

$$
A_{0}^{* *} x(y)=x\left(A_{0}^{*} y\right)=(J z)\left(A_{0}^{*} y\right)=\left(z, A_{0}^{*} y\right) .
$$

To make full use of the above observation, we need the following characterisation, for a Banach space $Z$, of those operators $C \in L(Z)$ whose range is closed, which is a standard corollary of the open mapping theorem (applied to the injective operator $z+\operatorname{ker} C \mapsto C z$ from $Z / \operatorname{ker} C$ to $Z$, also see [34, Theorem XI.2.1]): that

$$
C(Z) \text { is closed } \Leftrightarrow \quad \exists c>0 \text { s.t. }\|C z\| \geq c \inf _{y \in \operatorname{ker} C}\|z-y\|, \forall z \in Z .
$$

We also need the following consequence of the above characterisation.

LEMma 6.13. Suppose that $Z$ is a Banach space, $Z_{0}$ is a closed subspace of $Z$, $C\left(Z_{0}\right) \subset Z_{0}$, and set $C_{0}:=\left.C\right|_{Z_{0}}$. If the range of $C$ is closed and $\operatorname{ker} C=\operatorname{ker} C_{0}$ (i.e. $\operatorname{ker} C \subset Z_{0}$ ), then the range of $C_{0}$ is also closed. 
Proof. If the conditions of the lemma are satisfied then, by 6.11), there exists $c>0$ such that $\|C z\| \geq c \inf _{y \in \operatorname{ker} C}\|z-y\|, z \in Z$. But, since $Z_{0} \subset Z$ and $\operatorname{ker} C=\operatorname{ker} C_{0}$, this implies that $\left\|C_{0} z\right\| \geq c \inf _{y \in \operatorname{ker} C_{0}}\|z-y\|, z \in Z_{0}$, so that the range of $C_{0}$ is closed.

Corollary 6.14. If $A \in S(Y) \cap L_{0}(Y)$ and $A_{0}$ is semi-Fredholm with $\alpha\left(A_{0}\right)<$ $\infty$, then $A$ is semi-Fredholm and $\operatorname{ker} A=\operatorname{ker} A_{0}$.

Proof. If the conditions of the lemma are satisfied then, from standard results on Fredholm operators (e.g. [38]), we have that $A_{0}^{*}$ and $A_{0}^{* *}$ are also semi-Fredholm, and $\alpha\left(A_{0}\right)=\beta\left(A_{0}^{*}\right)=\alpha\left(A_{0}^{* *}\right)$. Applying Lemma 6.12, it follows that $\alpha\left(A_{0}\right)=$ $\alpha(A)=\alpha\left(A_{0}^{* *}\right)$. Further, since $\alpha\left(A_{0}\right)$ is finite and $\operatorname{ker} A_{0} \subset \operatorname{ker} A, \operatorname{ker} \check{A} \subset \operatorname{ker} A_{0}^{* *}$, it follows that $\operatorname{ker} A=\operatorname{ker} A_{0}$ and that $\operatorname{ker} \check{A}=\operatorname{ker} A_{0}^{* *}$. Applying Lemma 6.13 . since the range of $A_{0}^{* *}$ is closed it follows that the range of $\check{A}$ is closed and so $A(Y)$ is closed, and $A$ is semi-Fredholm.

We will prove the converse result only in the case when $U$ has a predual space $U^{\triangleleft}$ and $A$ has a preadjoint $A^{\triangleleft}$ on $Y^{\triangleleft}:=\ell^{1}\left(\mathbb{Z}^{N}, U^{\triangleleft}\right)$. Recall that, given a Banach space $X$, the Banach space $X^{\triangleleft}$ is said to be a predual space of $X$ if $X$ is isometrically isomorphic to $\left(X^{\triangleleft}\right)^{*}$. If $X$ has a predual space $X^{\triangleleft}$ then $B^{\triangleleft} \in L\left(X^{\triangleleft}\right)$ is said to be a preadjoint of $B \in L(X)$ if $\left(B^{\triangleleft}\right)^{*}=B$. If the Banach space $U$ has a predual space $U^{\triangleleft}$ then a predual space of $Y=\ell^{\infty}\left(\mathbb{Z}^{N}, U\right)$ is $Y^{\triangleleft}=\ell^{1}\left(\mathbb{Z}^{N}, U^{\triangleleft}\right)$. It is well-known that, if $X$ is a Banach space which has a predual $X^{\triangleleft}$ and $B \in L(X)$, then the following statements are equivalent:

(i) $B$ has a preadjoint $B^{\triangleleft} \in L\left(X^{\triangleleft}\right)$.

(ii) The adjoint $B^{*}$ maps $X^{\triangleleft}$, understood as a subspace of its second dual $\left(X^{\triangleleft}\right)^{* *}=X^{*}$, into itself.

(iii) $B$ is continuous in the weak* topology on $X$.

Recalling the isometry $J^{\triangleleft}: Y^{\triangleleft} \rightarrow Y_{1}$ introduced above, let $\check{Y}^{\triangleleft}=J^{\triangleleft}\left(Y^{\triangleleft}\right) \subset Y_{1}=$ $Y_{0}^{*}$, so that $\check{Y}^{\triangleleft}$ is isometrically isomorphic to $Y^{\triangleleft}$. For $A^{\triangleleft} \in L\left(Y^{\triangleleft}\right)$ let $\check{A}^{\triangleleft} \in L\left(\check{Y}^{\triangleleft}\right)$ be defined by $\check{A}^{\triangleleft}=J^{\triangleleft} A^{\triangleleft}\left(J^{\triangleleft}\right)^{-1}$.

Lemma 6.15. If $A \in L_{0}(Y), U$ has a predual $U^{\triangleleft}$ and $A$ a preadjoint $A^{\triangleleft} \in$ $L\left(Y^{\triangleleft}\right)$, then $A_{0}^{*}\left(\check{Y}^{\triangleleft}\right) \subset \check{Y}^{\triangleleft}$ and $\check{A}^{\triangleleft}=\left.A_{0}^{*}\right|_{Y^{\triangleleft}}$, so that $\operatorname{ker} \check{A}^{\triangleleft} \subset \operatorname{ker} A_{0}^{*}$.

Proof. For $x \in \check{Y}^{\triangleleft}$ and $y \in Y_{0}$, where $z:=\left(J^{\triangleleft}\right)^{-1} x \in Y^{\triangleleft}$, using [6.9),

$$
\check{A}^{\triangleleft} x(y)=\left(y, \check{A}^{\triangleleft} x\right)=y\left(A^{\triangleleft} z\right)=A y(z)=(A y, x) .
$$

Also,

$$
A_{0}^{*} x(y)=x\left(A_{0} y\right)=\left(A_{0} y, x\right)=(A y, x)
$$

Let $J_{1}: Y_{1} \rightarrow Y^{*}$ be defined by

$$
J_{1} x(y):=J y(x)=(y, x), \quad x \in Y_{1}, y \in Y .
$$

It is easy to check that $J_{1}$ is also an isometry. Let $\check{Y}_{1}:=J_{1}\left(Y_{1}\right) \subset Y^{*}$, which is isometrically isomorphic to $Y_{1}$. For $A_{1} \in L\left(Y_{1}\right)$ let $\check{A}_{1} \in L\left(\check{Y}_{1}\right)$ be defined by $\check{A}_{1}:=J_{1} A_{1} J_{1}^{-1}$. 
Lemma 6.16. If $A \in S(Y) \cap L_{0}(Y)$, then $A^{*}\left(\check{Y}_{1}\right) \subset \check{Y}_{1}$ and $\check{A}_{0}^{*}=\left.A^{*}\right|_{\check{Y}_{1}}$, so that $\operatorname{ker} \check{A}_{0}^{*} \subset \operatorname{ker} A^{*}$.

Proof. For $x \in \check{Y}_{1}, y \in Y$, where $z=J_{1}^{-1} x \in Y_{1}$,

$$
\check{A}_{0}^{*} x(y)=J_{1}\left(A_{0}^{*} z\right)(y)=\left(y, A_{0}^{*} z\right)=(A y, z),
$$

by Lemma 6.11. Also,

$$
A^{*} x(y)=x(A y)=J_{1} z(A y)=(A y, z) .
$$

We note that if the conditions of Lemmas 6.15 and 6.16 are satisfied, then

$$
\alpha\left(A^{\triangleleft}\right) \leq \alpha\left(A_{0}^{*}\right) \leq \alpha\left(A^{*}\right) .
$$

TheOREm 6.17. Suppose that $A \in S(Y) \cap L_{0}(Y), U$ has a predual $U^{\triangleleft}$ and $A$ has a preadjoint $A^{\triangleleft} \in L\left(Y^{\triangleleft}\right)$. Then $A$ is Fredholm if and only if $A_{0}$ is Fredholm and, if they are both Fredholm, then $\alpha\left(A_{0}\right)=\alpha(A), \beta\left(A_{0}\right)=\beta(A)$, and $\operatorname{ker} A=\operatorname{ker} A_{0}$.

Proof. Suppose first that $A_{0}$ is Fredholm . Then, by Corollary 6.14, $A$ is semiFredholm and $\operatorname{ker} A=\operatorname{ker} A_{0}$. This implies that $A^{\triangleleft}$ and $A^{*}$ are also semi-Fredholm, and so, and using 6.12,

$$
\beta(A)=\alpha\left(A^{\triangleleft}\right) \leq \alpha\left(A_{0}^{*}\right)=\beta\left(A_{0}\right),
$$

so that $A$ is Fredholm. Moreover,

$$
\beta(A)=\alpha\left(A^{*}\right) \geq \alpha\left(A_{0}^{*}\right)=\beta\left(A_{0}\right)
$$

so $\beta(A)=\beta\left(A_{0}\right)$.

Conversely, if $A$ is Fredholm then so are $A^{\triangleleft}$ and $A^{*}$ and $\alpha\left(A^{\triangleleft}\right)=\beta(A)=\alpha\left(A^{*}\right)$. Thus, by 6.12), $\alpha\left(\check{A}_{0}^{*}\right)=\alpha\left(A_{0}^{*}\right)=\alpha\left(A^{*}\right)$ is finite and so it follows from Lemma 6.16 that $\operatorname{ker} A_{0}^{*}=\operatorname{ker} A^{*}$. Applying Lemma 6.13 we see that the range of $\check{A}_{0}^{*}$ is closed, so that the range of $A_{0}^{*}$ is closed and $A_{0}^{*}$ is semi-Fredholm. Thus $A_{0}$ is also semi-Fredholm, with $\beta\left(A_{0}\right)=\alpha\left(A_{0}^{*}\right)=\alpha\left(A^{*}\right)<\infty$. But also $\alpha\left(A_{0}\right) \leq \alpha(A)$ is finite, so $A_{0}$ is Fredholm.

Note that the above theorem and its proof simplifies greatly if the Banach space $U$ is reflexive, in particular if $U$ is finite dimensional. For then we can choose $U^{\triangleleft}=U^{*}$ so that $Y^{\triangleleft}=Y_{1}$ and $Y_{0}^{* *}=Y$. Note also that, if the conditions of the above theorem hold, in particular if $A$ has a preadjoint, then the above theorem implies that $A$ is invertible if and only if $A_{0}$ is invertible. But even without existence of a preadjoint, we can prove this result in some cases; an observation which will be useful to us later.

Lemma 6.18. If $A \in S(Y) \cap L_{0}(Y)$ and $A$ is invertible, then $A_{0}$ is invertible.

Proof. If $A$ is invertible then $A_{0}$ is injective and it follows from Lemma 6.13 that the range of $A_{0}$ is closed. Further, since $A$ is the transpose of $A_{0}^{*}$ with respect to the dual system $\left(Y, Y_{1}\right)$ it follows (see e.g. [38]) that $0=\beta(A) \geq \alpha\left(A_{0}^{*}\right)=\beta\left(A_{0}\right)$. Thus $A_{0}$ is surjective.

Lemma 6.19. Suppose that $A \in L(Y, \mathcal{P})$ or that $A=I+K$ with $K \in S(Y) \cap$ $M(Y) \cap L_{0}(Y)$, and suppose that $A_{0}$ is invertible. Then $A$ is invertible. 
Proof. If the conditions of the lemma apply then, by Corollary 6.14 $A$ is injective. In the case that $A \in L(Y, \mathcal{P})$ then $A_{0} \in L\left(Y_{0}, \mathcal{P}\right)$ by Lemma 3.18 and since $L\left(Y_{0}, \mathcal{P}\right)$ is inverse closed (Theorem 1.1.9 of [74]), we have that $A_{0}^{-1} \in S\left(Y_{0}\right)$. This holds also by a modification of the proof of Lemma 3.33 in the case that $A=I+K$ with $K \in S(Y) \cap M(Y) \cap L_{0}(Y)$. For if $\left(x_{n}\right) \subset Y_{0}, x \in Y_{0}$, and $x_{n} \stackrel{s}{\rightarrow} x$ then, defining $y_{n}:=A_{0}^{-1} x_{n}$

$$
y_{n}+K y_{n}=x_{n}
$$

holds, and since $K \in M(Y)$ there exists a subsequence $\left(y_{n_{m}}\right)$ and $y \in Y$ such that $x_{n_{m}}-K y_{n_{m}} \stackrel{s}{\rightarrow} y$. From 6.13 it follows that $y_{n_{m}} \stackrel{s}{\rightarrow} y$. Since $K \in S(Y)$, it follows that $x_{n_{m}}-K y_{n_{m}} \stackrel{s}{\rightarrow} x-K y$. Thus $y=x-K y$, i.e. $A y=x$. Note that, by injectivity of $A$, there is only one $y \in Y$ with $A y=x$ and that is $y=A_{0}^{-1} x \in Y_{0}$. We have shown that $y_{n}=A_{0}^{-1} x_{n}$ has a subsequence strictly converging to $y=A_{0}^{-1} x$. By the same argument, every subsequence of $y_{n}$ has a subsequence strictly converging to $y$. Thus $A_{0}^{-1} x_{n} \stackrel{s}{\rightarrow} A_{0}^{-1} x$. So $A_{0}^{-1} \in S\left(Y_{0}\right)$.

Let $B \in S(Y)$ be the unique extension of $A_{0}^{-1}$ from $Y_{0}$ to $Y$, which exists by Lemma 3.18 . Then, for every $x \in Y$,

$$
B A x=s-\lim _{n \rightarrow \infty} B A P_{n} x=s-\lim A_{0}^{-1} A_{0} P_{n} x=x
$$

and, similarly, $A B x=x$. So $A$ is invertible.

Corollary 6.20. For $A \in L(Y, \mathcal{P})$ it holds that $A$ is invertible iff $A_{0}$ is invertible. When both are invertible, then $\left(A_{0}\right)^{-1}=\left.\left(A^{-1}\right)\right|_{Y_{0}}$.

Proof. The first sentence follows immediately from the previous two lemmas, and the equality concerning the two inverses is obvious if both $A$ and $A_{0}$ are invertible.

\subsection{Band-Dominated Operators}

Suppose $Y$ is one of the spaces $Y^{p}$ with $p \in\{0\} \cup[1, \infty]$ and $\mathcal{V}$ is given by 6.1). For $m \in \mathbb{Z}^{N}$ let $E_{m}: U \rightarrow Y$ and $R_{m}: Y \rightarrow U$ be extension and restriction operators, defined by $E_{m} y=(\ldots, 0, y, 0, \ldots)$, for $y \in U$, with the $y$ standing at the $m$ th place in the sequence, and by $R_{m} x=x(m)$, for $x=(x(m))_{m \in \mathbb{Z}^{N}} \in Y$. To every operator $A \in L(Y)$ we can associate a matrix $[A]=\left[a_{i j}\right]_{i, j \in \mathbb{Z}^{N}}$ with entries $a_{i j}=R_{i} A E_{j} \in L(U)$. For $x \in \tilde{Y}=\cup_{n \in \mathbb{N}} P_{n}(Y)$, we have that the $i$ th entry of $A x$ is

$$
(A x)(i)=\sum_{j \in \mathbb{Z}^{N}} a_{i j} x(j), \quad i \in \mathbb{Z}^{N} .
$$

Since $Y_{0}$ is the norm closure of $\tilde{Y}$, clearly the entries of $[A]$ determine $\left.A\right|_{Y_{0}}$ uniquely, and so determine $A$ if $Y=Y_{0}$, i.e. if $p \in\{0\} \cup[1, \infty)$. In the case $p=\infty,[A]$ determines $A$ if $A \in S(Y)$, for then $A x$ is given by $\lim _{n \rightarrow \infty} A P_{n} x$, the limit taken in the $s$ sense.

Let $B O(Y)$ denote the set of all operators $A \in L(Y, \mathcal{P})$ such that $[A]$ is a band matrix, that is, for some $w \in \mathbb{N}_{0}$ called the band-width of $A, a_{i j}=0$ if $|i-j|>w$.

Recall that, for $b=(b(m))_{m \in \mathbb{Z}^{N}} \in \ell^{\infty}\left(\mathbb{Z}^{N}, L(U)\right)$, the multiplication operator $M_{b} \in L(Y)$ is defined by (4.4). In terms of multiplication operators, an alternative 
characterisation of $B O(Y)$ is the following [51]: that $A \in L(Y)$ is a band operator of band-width $w$ iff

$$
A=\sum_{|k| \leq w} M_{b_{k}} V_{k}
$$

with some $b_{k} \in \ell^{\infty}\left(\mathbb{Z}^{N}, L(U)\right)$ for every $|k| \leq w$. For $A$ given by 6.15 the matrix representation $[A]$ has $a_{i j}=b_{i-j}(i)$ for $|i-j| \leq w$ and $a_{i j}=0$, otherwise. Since $\mathcal{V} \subset L(Y, \mathcal{P})$ and, clearly, $M_{b} \in L(Y, \mathcal{P})$ for $b \in \ell^{\infty}\left(\mathbb{Z}^{N}, L(U)\right)$, every $A$ of the form 6.15 is in $L(Y, \mathcal{P})$.

The linear space $B O(Y)$ is an algebra but is not closed with respect to the norm in $L(Y)$. By taking the closure of $B O(Y)$ in the operator norm of $L(Y)$ we obtain the Banach algebra $B D O(Y)$. We refer to the elements of $B D O(Y)$ as banddominated operators. It should be noted that $B D O\left(Y^{p}\right)$ depends on the exponent $p$ of the underlying space, while $B O\left(Y^{p}\right)$ does not. Since $L(Y, \mathcal{P})$ is closed in $L(Y)$, and $B O(Y) \subset L(Y, \mathcal{P})$, it follows that $B D O(Y) \subset L(Y, \mathcal{P})$.

As a consequence of (5.13), an operator $A \in B O(Y)$, which has the form (6.15), is a rich operator iff the multiplication operators $M_{b_{k}}$ are rich for all $|k| \leq w$, i.e. iff (see Example 6.6) the set $\left\{V_{i} b_{k}: i \in \mathbb{Z}^{N}\right\}$ is relatively sequentially compact in the strict topology on $Y^{\infty}(L(U))$ for every $k$. Further, if $A \in B D O(Y)$, in which case $A_{n} \rightrightarrows A$ for some $\left(A_{n}\right) \subset B O(Y), A$ is rich if each $A_{n}$ is rich.

LEmma 6.21. [74, Corollary 2.1.17] If $U$ is finite-dimensional then every banddominated operator is rich.

For band-dominated operators the notions of invertibility at infinity (Definition 5.1) and $\mathcal{P}$-Fredholmness (Remark 5.2) coincide.

Lemma 6.22. If $A \in B D O(Y)$, then the following statements are equivalent.

(i) $A$ is invertible at infinity.

(ii) There exist $B \in B D O(Y)$ and $T_{1}, T_{2} \in K(Y, \mathcal{P})$ such that 5.1 holds.

(iii) $A$ is $\mathcal{P}$-Fredholm.

Proof. For $Y^{p}$ with $1 \leq p \leq \infty$ this is precisely Proposition 2.10 of [51] (the key idea, written down for $Y^{2}$, is from [73, Proposition 2.6]). The proof from [51] literally transfers to $Y^{0}$.

For band-dominated operators we have also the following result; in fact the equivalence of (i), (ii) and (iii) in the next lemma is true even for arbitrary $K \in L(Y)$.

Lemma 6.23. If $K \in B D O(Y)$, with $[K]=\left[\kappa_{i j}\right]_{i, j \in \mathbb{Z}^{N}}$ the matrix representation of $K$, then the following statements are equivalent.

(i) $\left\{V_{-k} K V_{k}: k \in \mathbb{Z}^{N}\right\} \cup \sigma^{\mathrm{op}}(K)$ is uniformly Montel on $(Y, s)$.

(ii) $\left\{V_{-k} K V_{k}: k \in \mathbb{Z}^{N}\right\}$ is uniformly Montel on $(Y, s)$.

(iii) $\left(V_{-k} K V_{k}\right)_{k \in \mathbb{Z}^{N}}$ is asymptotically Montel on $(Y, s)$ and $K \in M(Y)$.

(iv) The set $\left\{\kappa_{i j}: i, j \in \mathbb{Z}^{N}\right\} \subset L(U)$ is collectively compact.

(v) The set $\left\{\kappa_{i j}: i, j \in \mathbb{Z}^{N}, i-j=d\right\} \subset L(U)$ is collectively compact for every $d \in \mathbb{Z}^{N}$.

If $U$ is finite-dimensional, then (i)-(v) are also equivalent to:

(vi) The set $\left\{\kappa_{i j}: i, j \in \mathbb{Z}^{N}, i-j=d\right\} \subset L(U)$ is bounded for every $d \in \mathbb{Z}^{N}$. 
Proof. It is clear from the definitions that (i) $\Rightarrow$ (ii), (ii) $\Rightarrow$ (iii) and that (iv) $\Rightarrow$ (v). By Lemma 5.17, (ii) implies (i).

Suppose now that (iii) holds and that $h=(h(n))_{n=1}^{\infty} \subset \mathbb{Z}^{N}$ and that $\left(x_{n}\right) \subset Y$ is bounded. If $h$ does not have a subsequence that tends to infinity, then $h$ is bounded, and hence it has a subsequence that is constant. In the case that $h$ has a subsequence that tends to infinity, $\left(V_{-h(n)} K V_{h(n)} x_{n}\right)$ has a strictly convergent subsequence since $\left(V_{-k} K V_{k}\right)$ is asymptotically Montel. In the case that $h$ has a constant subsequence, $\left(V_{-h(n)} K V_{h(n)} x_{n}\right)$ has a strictly convergent subsequence since $K \in M(Y)$. In either case, we have shown that (ii) holds.

Next suppose that (ii) holds and that $i=(i(n))_{n=1}^{\infty} \subset \mathbb{Z}^{N}, j=(j(n))_{n=1}^{\infty} \subset \mathbb{Z}^{N}$, and that $\left(u_{n}\right) \subset U$ is bounded. For $n \in \mathbb{Z}^{N}$ define $\left(x_{n}\right) \in Y$ by setting the $i(n)-j(n)$ entry of $x_{n}$ equal to $u_{n}$ and setting the other entries to zero. Then $\left(x_{n}\right)$ is bounded and the zeroth entry of $\left(V_{-i(n)} K V_{i(n)} x_{n}\right)$ is $\kappa_{i(n), j(n)} u_{n}$. Since $\left\{V_{-k} K V_{k}: k \in \mathbb{Z}^{N}\right\}$ is uniformly Montel, $\left(V_{-i(n)} K V_{i(n)} x_{n}\right)(0)=\kappa_{i(n), j(n)} u_{n}$ has a convergent subsequence. Since $i, j$, and $\left(u_{n}\right)$ were arbitrary sequences, we have shown that (iv) holds.

Finally, suppose that (v) holds. Then the set $\left\{\kappa_{i j}: i, j \in \mathbb{Z}^{N},|i-j| \leq w\right\}$ is collectively compact for every $w \in \mathbb{N}$. For every $M \in \mathbb{N}$, every $h=(h(n))_{n=1}^{\infty} \subset$ $\mathbb{Z}^{N}$, and every bounded sequence $\left(x_{n}\right) \subset Y$, we have that the $i$ th component of $\left(V_{-h(n)} K V_{h(n)} P_{M} x_{n}\right)$ is

$$
\sum_{|j| \leq M} \kappa_{i+h(n), j+h(n)} x_{n}(j)
$$

Since $\left\{\kappa_{i j}: i, j \in \mathbb{Z}^{N},|i-j| \leq w\right\}$ is collectively compact for each $w$, it follows that the $i$ th component of $\left(V_{-h(n)} K V_{h(n)} P_{M} x_{n}\right)$ has a convergent subsequence for every $M \in \mathbb{Z}$. Thus, by a diagonal argument, $\left(V_{-h(n)} K V_{h(n)} P_{M} x_{n}\right)$ has a strictly convergent subsequence, for every $M \in \mathbb{N}$. Again by a diagonal argument, we can find subsequences of $h$ and $\left(x_{n}\right)$, which we will denote again by $h$ and $\left(x_{n}\right)$, such that $\left(V_{-h(n)} K V_{h(n)} P_{n} x_{n}\right)$ converges strictly to some $x \in Y$, so that $P_{m} V_{-h(n)} K V_{h(n)} P_{n} x_{n} \rightarrow P_{m} x$ as $n \rightarrow \infty$, for each $m$. Now [74, since $K$ is band-dominated, it holds for every $m \in \mathbb{N}$ that $P_{m} V_{-k} K V_{k} Q_{n} \rightrightarrows 0$ as $n \rightarrow \infty$, uniformly in $k \in \mathbb{Z}^{N}$. Thus $P_{m} V_{-h(n)} K V_{h(n)} x_{n} \rightarrow P_{m} x$, for every $m \in \mathbb{N}$, so that $V_{-h(n)} K V_{h(n)} x_{n} \stackrel{s}{\rightarrow} x$. We have shown that (ii) holds.

The equivalence of (vi) and (v) under the condition $\operatorname{dim} U<\infty$ is obvious since, in that case, a subset of $U$ is relatively compact iff it is bounded.

For brevity, and because we will frequently refer to this class of operators in what follows, let us denote the set of all operators $K \in B D O(Y)$ which are subject to the (equivalent) properties (i)-(v) of Lemma 6.23 by $U M(Y)$.

LEMma 6.24. The following statements hold.

(a) The set $U M(Y)$ is a Banach subspace of $B D O(Y) \cap M(Y)$.

(b) In particular, $U M(Y)=B D O(Y)$ if $U$ is finite-dimensional.

(c) If $K \in U M(Y)$ and $A \in B D O(Y)$, then $K A \in U M(Y)$.

(d) If $M_{b}$ is rich and $K \in U M(Y)$, then $M_{b} K \in U M(Y)$. 
Proof. (a): By its definition, we have that $U M(Y) \subset B D O(Y)$, and from Lemma 6.23 (iii) we get that $U M(Y) \subset M(Y)$. For the rest of this proof, we will use property (ii) from Lemma 6.23 to characterise the set $U M(Y)$.

If $S, T \in U M(Y)$ and $\lambda \in \mathbb{C}$, then clearly $\lambda S+T \in U M(Y)$ since

$$
\left\{V_{-k}(\lambda S+T) V_{k}: k \in \mathbb{Z}^{N}\right\} \subset \lambda\left\{V_{-k} S V_{k}: k \in \mathbb{Z}^{N}\right\}+\left\{V_{-k} T V_{k}: k \in \mathbb{Z}^{N}\right\}
$$

is uniformly Montel.

If $T_{1}, T_{2}, \ldots \in U M(Y)$ are such that $T_{n} \rightrightarrows T$, then also $T \in U M(Y)$. To see this, take a sequence $k=(k(1), k(2), \ldots) \subset \mathbb{Z}^{N}$ and a sequence $\left(x_{1}, x_{2}, \ldots\right) \subset Y$ with $\mu:=\sup \left\|x_{\ell}\right\|<\infty$. By a simple diagonal argument, we can pick a strictly monotonously increasing sequence $s=(s(1), s(2), \ldots) \subset \mathbb{N}$ such that

$$
V_{-k(s(\ell))} T_{n} V_{k(s(\ell))} x_{s(\ell)}
$$

converges strictly as $\ell \rightarrow \infty$ for every $n \in \mathbb{N}$. Let us denote the strict limit by $y_{n}$, respectively. From

$$
\left\|y_{n_{1}}-y_{n_{2}}\right\| \leq \sup _{\ell}\left\|V_{-k(s(\ell))}\left(T_{n_{1}}-T_{n_{2}}\right) V_{k(s(\ell))} x_{s(\ell)}\right\| \leq\left\|T_{n_{1}}-T_{n_{2}}\right\| \cdot \mu
$$

we see that $\left(y_{n}\right)$ is a Cauchy sequence in $Y$ and therefore converges, to $y \in Y$, say. But then $V_{-k(s(\ell))} T V_{k(s(\ell))} x_{s(\ell)} \stackrel{s}{\rightarrow} y$ as $\ell \rightarrow \infty$. Indeed, for all $M, n \in \mathbb{N}$,

$$
\begin{aligned}
\left\|P_{M}\left(V_{-k(s(\ell))} T V_{k(s(\ell))} x_{s(\ell)}-y\right)\right\| & \\
\leq \| P_{M}\left(V_{-k(s(\ell))} T_{n} V_{k(s(\ell))} x_{s(\ell)}\right. & \left.-y_{n}\right)\|+\| P_{M}\left(y_{n}-y\right) \| \\
& +\left\|P_{M}\left(V_{-k(s(\ell))}\left(T-T_{n}\right) V_{k(s(\ell))} x_{s(\ell)}\right)\right\| \\
\leq \| P_{M}\left(V_{-k(s(\ell))} T_{n} V_{k(s(\ell))} x_{s(\ell)}\right. & \left.-y_{n}\right)\|+\| y_{n}-y\|+\| T-T_{n} \| \cdot \mu
\end{aligned}
$$

holds. But, for every choice of $M, n \in \mathbb{N}$, the first term goes to zero as $\ell \rightarrow \infty$, and the second and third term can be made as small as desired by choosing $n$ sufficiently large.

(b): If $K \in B D O(Y)$ then property (vi) of Lemma 6.23 is automatically the case. Since this is equivalent to properties (i)-(v) of the same lemma if $U$ is finitedimensional, we get that $K \in U M(Y)$ then.

(c), (d): Let $K \in U M(Y), A \in B D O(Y)$ and $b \in Y^{\infty}(L(U))$ such that $M_{b}$ is rich. Take a sequence $k=(k(1), k(2), \ldots) \subset \mathbb{Z}^{N}$ and a bounded sequence $\left(x_{1}, x_{2}, \ldots\right) \subset Y$. Now, for every $\ell \in \mathbb{N}$, put $y_{\ell}:=V_{-k(\ell)} A V_{k(\ell)} x_{\ell}$. Since $\left(y_{\ell}\right)$ is bounded, $\left\{V_{-m} K V_{m}: m \in \mathbb{Z}^{N}\right\}$ is uniformly Montel and $\left\{V_{-m} b: m \in \mathbb{Z}^{N}\right\}$ is relatively sequentially compact in the strict topology on $Y^{\infty}\left(L(U)\right.$ ) (since $M_{b}$ is rich, see Example 6.6), we can pick a strictly monotonously increasing sequence $s=(s(1), s(2), \ldots) \subset \mathbb{N}$ such that both $V_{-k(s(\ell))} K V_{k(s(\ell))} y_{s(\ell)}$ and $V_{-k(s(\ell))} b$ converge strictly as $\ell \rightarrow \infty$. But then $V_{-k(s(\ell))}\left(M_{b} K A\right) V_{k(s(\ell))} x_{s(\ell)}$ converges strictly as $\ell \rightarrow \infty$ since, for every $m \in \mathbb{N}$,

$$
\begin{aligned}
& P_{m} V_{-k(s(\ell))}\left(M_{b} K A\right) V_{k(s(\ell))} x_{s(\ell)} \\
& \quad=P_{m}\left(V_{-k(s(\ell))} M_{b} V_{k(s(\ell))}\right)\left(V_{-k(s(\ell))} K V_{k(s(\ell))}\right)\left(V_{-k(s(\ell))} A V_{k(s(\ell))} x_{s(\ell)}\right) \\
& \quad=M_{P_{m} V_{-k(s(\ell))}} P_{m}\left(V_{-k(s(\ell))} K V_{k(s(\ell))} y_{s(\ell)}\right)
\end{aligned}
$$

converges in norm as $\ell \rightarrow \infty$, i.e. $M_{b} K A \in U M(Y)$. 
The purpose of the following two lemmas is to prove that, for every $A \in$ $L\left(Y^{1}(U)\right)$, the operator spectra $\sigma^{\mathrm{op}}(A) \subset L\left(Y^{1}(U)\right)$ and $\sigma^{\mathrm{op}}\left(A^{*}\right) \subset L\left(\left(Y^{1}(U)\right)^{*}\right)=$ $L\left(Y^{\infty}\left(U^{*}\right)\right)$ correspond elementwise in terms of adjoints.

Lemma 6.25. If $A \in L\left(Y^{1}(U)\right)$, then

$$
\sigma^{\mathrm{op}}\left(A^{*}\right)=\left\{B^{*}: B \in \sigma^{\mathrm{op}}(A)\right\} .
$$

Proof. It is a standard result that $B=A_{h} \in \sigma^{\mathrm{op}}(A)$ implies $B^{*}=\left(A_{h}\right)^{*}=\left(A^{*}\right)_{h} \in$ $\sigma^{\text {op }}\left(A^{*}\right)$ (see, e.g. [51, Proposition 3.4 e]).

For the reverse implication, suppose $C \in \sigma^{\mathrm{op}}\left(A^{*}\right) \subset L\left(Y^{\infty}\left(U^{*}\right)\right)$. Then

$$
\left(V_{-h(m)} A V_{h(m)}\right)^{*}=V_{-h(m)} A^{*} V_{h(m)} \stackrel{\mathcal{P}}{\rightarrow} C
$$

as $m \rightarrow \infty$ for some sequence $h(1), h(2), \ldots \rightarrow \infty$ in $\mathbb{Z}^{N}$. We will show in Lemma 6.26 that then $C=B^{*}$ and $V_{-h_{m}} A V_{h_{m}} \stackrel{\mathcal{P}}{\rightarrow} B$, i.e. $B \in \sigma^{\mathrm{op}}(A)$.

Lemma 6.26. The set of operators in $L\left(Y^{\infty}\left(U^{*}\right)\right)$ that possess a preadjoint in $L\left(Y^{1}(U)\right)$ is sequentially closed under $\mathcal{P}$-convergence; that is, if $A_{1}, A_{2}, \ldots \in$ $L\left(Y^{1}(U)\right)$ and $A_{m}^{*} \stackrel{\mathcal{P}}{\rightarrow} C$ on $Y^{\infty}\left(U^{*}\right)$, then there is a $B \in L\left(Y^{1}(U)\right)$ such that $C=B^{*}$; moreover, $A_{m} \stackrel{\mathcal{P}}{\rightarrow} B$ on $Y^{1}(U)$.

Proof. From $A_{m}^{*} \stackrel{\mathcal{P}}{\rightarrow} C$ in $L\left(Y^{\infty}\left(U^{*}\right)\right)$ and Lemma 4.2 we get that there is a $M>0$ such that

$$
\left\|A_{m}\right\|=\left\|A_{m}^{*}\right\| \leq M, \quad m \in \mathbb{N} .
$$

Moreover, for every $k \in \mathbb{N}$, it holds that

$$
P_{k}\left(A_{m}^{*}-C\right) \rightrightarrows 0 \quad \text { as } \quad m \rightarrow \infty .
$$

So we get that $\left(P_{k} A_{m}^{*}\right)_{m=1}^{\infty}$ is a Cauchy sequence in $L\left(Y^{\infty}\left(U^{*}\right)\right)$ and therefore $\left(A_{m} P_{k}\right)_{m=1}^{\infty}$ is one in $L\left(Y^{1}(U)\right)$, for every fixed $k \in \mathbb{N}$. Denote the norm-limit of the latter sequence by $B_{k} \in L\left(Y^{1}(U)\right)$. As a consequence of $(6.16)$ we get that

$$
\left\|B_{k}\right\|=\left\|\lim _{m \rightarrow \infty} A_{m} P_{k}\right\| \leq \sup _{m}\left\|A_{m} P_{k}\right\| \leq M, \quad k \in \mathbb{N} .
$$

From $A_{m} P_{k} \rightrightarrows B_{k}$ we get that $B_{k} P_{k}=B_{k}$ and, even more than this, that

$$
B_{r} P_{k}=\lim _{m \rightarrow \infty} A_{m} P_{r} P_{k}=\lim _{m \rightarrow \infty} A_{m} P_{k}=B_{k}, \quad r \geq k .
$$

We will now show that the sequence $B_{1}, B_{2}, \ldots$ strongly converges in $Y^{1}(U)$. Therefore, take an arbitrary $x \in Y^{1}(U)$ and let us verify that $\left(B_{m} x\right)$ is a Cauchy sequence in $Y^{1}(U)$. So choose some $\varepsilon>0$. Since $Q_{m} x \rightarrow 0$ on $Y^{1}(U)$, there is an $N \in \mathbb{N}$ such that

$$
\left\|Q_{N} x\right\|<\frac{\varepsilon}{2 M} .
$$

Now, for all $k, m \geq N$, the following holds

$$
\begin{aligned}
\left\|B_{k} x-B_{m} x\right\| & \leq\left\|\left(B_{k}-B_{m}\right) P_{N} x\right\|+\left\|\left(B_{k}-B_{m}\right) Q_{N} x\right\| \\
& \leq\left\|\left(B_{k} P_{N}-B_{m} P_{N}\right) x\right\|+\left\|B_{k}-B_{m}\right\| \cdot\left\|Q_{N} x\right\| \\
& \leq\left\|\left(B_{N}-B_{N}\right) x\right\|+\left(\left\|B_{k}\right\|+\left\|B_{m}\right\|\right) \cdot\left\|Q_{N} x\right\|<\varepsilon
\end{aligned}
$$


by 6.19, 6.18 and 6.20. Consequently, $\left(B_{m} x\right)$ is a Cauchy sequence in $Y^{1}(U)$. Let us denote its limit in $Y^{1}(U)$ by $B x$, thereby defining an operator $B \in L\left(Y^{1}(U)\right)$. Passing to the strong limit as $r \rightarrow \infty$ in 6.19, we get

$$
B P_{k}=B_{k}, \quad k \in \mathbb{N} \text {. }
$$

Summing up, we have $A_{m} P_{k} \rightrightarrows B_{k}=B P_{k}$, and hence $\left(A_{m}-B\right) P_{k} \rightrightarrows 0$ as $m \rightarrow \infty$, for all $k \in \mathbb{N}$. Passing to adjoints in the latter gives $P_{k}\left(A_{m}^{*}-B^{*}\right) \rightrightarrows 0$ in $L\left(Y^{\infty}\left(U^{*}\right)\right)$ as $m \rightarrow \infty$. If we subtract this from (6.17) we get $P_{k}\left(B^{*}-C\right)=0$ for all $k \in \mathbb{N}$, and consequently $C=B^{*}$, by Lemma 1.30 a) in [51]. From $A_{m}^{*} \stackrel{\mathcal{P}}{\rightarrow} C=B^{*}$ we then conclude

$$
\left\|\left(A_{m}-B\right) P_{k}\right\|=\left\|P_{k}\left(A_{m}^{*}-B^{*}\right)\right\| \rightarrow 0 \quad \text { as } \quad m \rightarrow \infty
$$

and

$$
\left\|P_{k}\left(A_{m}-B\right)\right\|=\left\|\left(A_{m}^{*}-B^{*}\right) P_{k}\right\| \rightarrow 0 \quad \text { as } \quad m \rightarrow \infty
$$

for every $k \in \mathbb{N}$, which, together with 6.16 and again Lemma 4.2 , proves $A_{m} \stackrel{\mathcal{P}}{\rightarrow} B$.

Our next statement is similar to Lemma 6.25, but with restriction from $Y$ to $Y_{0}$ instead of passing to the adjoint operator.

Lemma 6.27. If $A \in L(Y, \mathcal{P})$, then the limit operators of the restriction $A_{0}:=$ $\left.A\right|_{Y_{0}}$ are the restrictions of the limit operators of $A$; precisely,

$$
\sigma^{\mathrm{op}}\left(A_{0}\right)=\left\{\left.B\right|_{Y_{0}}: B \in \sigma^{\mathrm{op}}(A)\right\}
$$

In particular, the invertibility of all limit operators of $A_{0}$ in $Y_{0}$ with uniform boundedness of their inverses is equivalent to the same property for the limit operators of $A$ in $Y$.

Proof. The proof of 6.22 consists of two observations. The first one is that $\left.\left(V_{-\alpha} A V_{\alpha}\right)\right|_{Y_{0}}=V_{-\alpha} A_{0} V_{\alpha}$ for all $\alpha \in \mathbb{Z}^{N}$, and the second one is that $\left.A_{m}\right|_{Y_{0}} \stackrel{\mathcal{P}}{\rightarrow} A_{0}$ on $Y_{0}$ iff $A_{m} \stackrel{\mathcal{P}}{\rightarrow} A$ on $Y$, for all $A_{1}, A_{2}, \ldots \in L(Y, \mathcal{P})$ since

$$
\left\|P_{k}\left(\left.A_{m}\right|_{Y_{0}}-A_{0}\right)\right\|=\left\|\left.\left(P_{k}\left(A_{m}-A\right)\right)\right|_{Y_{0}}\right\|=\left\|P_{k}\left(A_{m}-A\right)\right\|
$$

and its symmetric counterpart hold for all $k \in \mathbb{N}$ by the norm equality in Lemma 3.18 The proof of the second sentence of the lemma now follows from 6.22, Corollary 6.20 and the norm equality in Lemma 3.18 again.

For the choice of $Y, \mathcal{P}$ and $\mathcal{V}$ we are making in this section, the operator spectrum of a rich band-dominated operator $A$ contains enough information to characterise the invertibility at infinity of $A$, which is the content of (iii) in the next theorem. In this theorem, for an operator $A \in L(Y)$, we denote the spectrum of $A$, i.e. the set of all $\lambda \in \mathbb{C}$ for which $\lambda I-A$ is not invertible, by $\operatorname{spec}(A)$. We denote by $\operatorname{spec}_{\text {ess }}(A)$ the essential spectrum of $A$, i.e. the set of $\lambda$ for which $\lambda I-A$ is not Fredholm . We also define, for $\varepsilon>0$, the $\varepsilon$-pseudospectrum of $A$, $\operatorname{spec}_{\varepsilon}(A)$, by

$$
\operatorname{spec}_{\varepsilon}(A):=\left\{\lambda \in \mathbb{C}: \lambda I-A \text { is not invertible or }\left\|(\lambda I-A)^{-1}\right\| \geq \varepsilon^{-1}\right\} .
$$

TheOREM 6.28. a) Let $A$ be a rich band-dominated operator on $Y=Y^{p}(U)$ with a Banach space $U$ and some $p \in\{0\} \cup[1, \infty]$. Then the following statements hold. 
(i) If $A$ is Fredholm and $p \neq \infty$ then $A$ is invertible at infinity;

(ii) If $A$ is invertible at infinity and either $U$ is finite-dimensional or $A=$ $C+K$ with $C \in B D O(Y)$ invertible and $K \in M(Y)$ then $A$ is Fredholm;

(iii) $A$ is invertible at infinity if and only if all limit operators of $A$ are invertible and their inverses are uniformly bounded;

(iv) The condition on uniform boundedness in (iii) is redundant if $p \in\{0,1, \infty\}$;

(v) It holds that $\operatorname{spec}(B) \subset \operatorname{spec}(A)$ for all $B \in \sigma^{\mathrm{op}}(A)$, indeed $\operatorname{spec}(B) \subset$ $\operatorname{spec}_{\text {ess }}(A)$, for $p \neq \infty$;

(vi) It holds that $\operatorname{spec}_{\varepsilon}(B) \subset \operatorname{spec}_{\varepsilon}(A)$, for all $B \in \sigma^{\mathrm{op}}(A)$ and $\varepsilon>0$.

b) In the case $p=\infty$ if, in addition, it holds that $U$ has a predual $U^{\triangleleft}$, and $A$ has a preadjoint, $A^{\triangleleft} \in L\left(Y^{\triangleleft}\right)$, where $Y^{\triangleleft}=Y^{1}\left(U^{\triangleleft}\right)$, then (i) and (v) also apply for $p=\infty$; that is, $A$ being Fredholm implies $A$ being invertible at infinity, so that $\operatorname{spec}(B) \subset \operatorname{spec}_{\mathrm{ess}}(A) \subset \operatorname{spec}(A)$ for all $B \in \sigma^{\mathrm{op}}(A)$.

REMARK 6.29 This theorem makes several additions and simplifications to previously known results: (i), and therefore (v), is probably new for $p=1$, and so is statement b). (ii) is a slight extension of Proposition 2.15 (together with the first column of Figure 4) of [51]. (iii) does not assume the existence of a preadjoint operator (unlike Theorem 1 in [49] and [51]) if $p=\infty$. (iv) is probably new for $p=0$. Also note that our Lemma 6.25 fills a gap in the proof of [51, Proposition 3.6 a)] that is used in the proof of [51, Theorem 3.109] to deal with the case $p=1$. (vi) was only known when $p=2$ and $U$ is a Hilbert space. For this setting, it follows from Theorem 6.3.8 (b) of $\mathbf{7 4}$ which, in fact, states the stronger result that the closure of the union of all $\operatorname{spec}_{\varepsilon}(B)$ with $B \in \sigma^{\mathrm{op}}(A)$ is equal to the $\varepsilon$-pseudospectrum of the coset of $A$ modulo $K(Y, \mathcal{P})$.

Proof of Theorem 6.28. b) Suppose the predual $U^{\triangleleft}$ and preadjoint $A^{\triangleleft}$ exist and that $A$ is Fredholm on $Y=Y^{\infty}(U)$. By Theorem 6.17 (note that $B D O(Y) \subset$ $\left.L(Y, \mathcal{P}) \subset S(Y) \cap L_{0}(Y)\right)$, we have that $A_{0}:=\left.A\right|_{Y_{0}}$ is Fredholm on $Y_{0}=Y^{0}(U)$. From Lemma 6.1 (i) (also see Remark 6.2) we get that then $A_{0}$ is invertible at infinity on $Y_{0}$, which, by Lemma 6.22 means that we have $A_{0} B_{0}=I+S_{0}$ and $B_{0} A_{0}=I+T_{0}$ for some $B_{0} \in L\left(Y_{0}, \mathcal{P}\right)$ and $S_{0}, T_{0} \in K\left(Y_{0}, \mathcal{P}\right)$. If we use Lemma 3.18 to extend both sides of these two equalities to operators on $Y$, then we get that $A$ is invertible at infinity on $Y$.

a) (iii) For $p \in\{0\} \cup(1, \infty)$ we refer the reader to [74, Theorem 2.2.1], and for $p=1$ (and also $p \in(1, \infty)$ ) to [51, Theorem 1]. It remains to study the case $p=\infty$. The 'if' part of statement (iii) is Proposition 3.16 in [51] (which does not use the existence of a preadjoint). For the 'only-if' part of (iii) we replace Proposition 3.12 from [51 (which needs the preadjoint) by the following argument. Suppose $A$ is invertible at infinity on $Y=Y^{\infty}$. By Lemma 6.22, there are $B \in B D O(Y) \subset$ $L(Y, \mathcal{P})$ and $S, T \in K(Y, \mathcal{P})$ with $A B=I+S$ and $B A=I+T$. Restricting both sides in both equalities to $Y_{0}$ we get that, by Lemma 3.18, $A_{0}:=\left.A\right|_{Y_{0}}$ is invertible at infinity on $Y_{0}$, which, by our result (iii) for $p=0$, implies that all limit operators of $A_{0}$ are invertible on $Y_{0}$ and their inverses are uniformly bounded. From Lemma 6.27 we now get that also all limit operators of $A$ are invertible on $Y$ and their inverses are uniformly bounded.

Statement (iv) for $p \in\{1, \infty\}$ is [51, Theorem 3.109]. Precisely, the part for $p=\infty$ follows immediately from [51, Proposition 3.108], and the $p=1$ part is a 
consequence of this and Lemma 6.25. Indeed, if all $B \in \sigma^{\mathrm{op}}(A)$ are invertible on $Y^{1}(U)$ then also all their adjoints $C=B^{*}$ are invertible on $Y^{\infty}\left(U^{*}\right)$, which, by Lemma 6.25, are all elements of $\sigma^{\mathrm{op}}\left(A^{*}\right)$. Since $A^{*} \in B D O\left(Y^{\infty}\left(U^{*}\right)\right)$ is rich as well, we know from the results about $p=\infty$ that

$$
\begin{aligned}
\sup _{B \in \sigma^{\circ \mathrm{p}}(A)}\left\|B^{-1}\right\| & =\sup _{B \in \sigma^{\mathrm{op}}(A)}\left\|\left(B^{-1}\right)^{*}\right\|=\sup _{B \in \sigma^{\mathrm{op}}(A)}\left\|\left(B^{*}\right)^{-1}\right\| \\
& =\sup _{C=B^{*} \in \sigma^{\mathrm{op}}\left(A^{*}\right)}\left\|C^{-1}\right\|<\infty
\end{aligned}
$$

since $B \in \sigma^{\mathrm{op}}(A)$ iff $C=B^{*} \in \sigma^{\mathrm{op}}\left(A^{*}\right)$, by Lemma 6.25. The statement (iv) for $p=0$ follows immediately from Lemma 6.27 (applied to the extension of $A$ ) and the result for $p=\infty$.

(i) For $p \in\{0\} \cup(1, \infty)$ this follows immediately from Lemma 6.1 (i) (it was already pointed out in Remark 6.2). So let $p=1$ and suppose $A$ is Fredholm on $Y=Y^{1}(U)$. Then $A^{*}$ is Fredholm on $Y^{\infty}\left(U^{*}\right)$. From part b) of this theorem it follows that $A^{*}$ is invertible at infinity on $Y^{\infty}\left(U^{*}\right)$. From (iii) we get that all limit operators of $A^{*}$ are invertible on $Y^{\infty}\left(U^{*}\right)$. By Lemma 6.25 this implies that all limit operators of $A$ are invertible on $Y^{1}(U)$, which, by (iii) and (iv), shows that $A$ is invertible at infinity.

(ii) Suppose $A$ is invertible at infinity. If $\operatorname{dim} U<\infty$ then Lemma 6.1 (ii) (also see Remark 6.2 implies that $A$ is Fredholm. Alternatively, suppose that $A=C+K$ with $C \in B D O(Y)$ invertible and $K \in M(Y)$. From $C \in B D O(Y) \subset L(Y, \mathcal{P})$ we get, by [74, Theorem 1.1.9], that $C^{-1} \in L(Y, \mathcal{P}) \subset S(Y)$. Moreover, $K=A-C \in$ $B D O(Y) \subset S(Y)$ implies that $K \in S(Y) \cap M(Y)$ so that $A$ is subject to the constraints in Theorem 5.4 which proves that $A$ is Fredholm.

(v) For arbitrary $\lambda \in \mathbb{C}, \lambda I-B \in \sigma^{\mathrm{op}}(\lambda I-A)$ iff $B \in \sigma^{\mathrm{op}}(A)$. So it suffices to show that Fredholmness of a rich band-dominated operator (for $p \neq \infty$ ) implies invertibility of its limit operators. But this is an immediate consequence of (i) and (iii).

(vi) From (iii) we know that, if $B \in \sigma^{\mathrm{op}}(A)$ and $\lambda I-B$ is not invertible then $\lambda I-A$ is not invertible (not even invertible at infinity). So suppose $\lambda I-B$ is invertible. If $\lambda I-A$ is not invertible then there is nothing to prove. If also $\lambda I-A$ is invertible then, by Theorem 5.12 (ix), which applies since $B \in \sigma^{\mathrm{op}}(A) \subset L(Y, \mathcal{P})$ as $A \in B D O(Y) \subset L(Y, \mathcal{P})$, it follows that

$$
\left\|(\lambda I-B)^{-1}\right\|=\frac{1}{\nu(\lambda I-B)} \leq \frac{1}{\nu(\lambda I-A)}=\left\|(\lambda I-A)^{-1}\right\| .
$$

Now we will combine the results of Theorems 6.3 and 6.28, Recall that the set $U M(Y)$ was introduced just before (and studied in) Lemma 6.24

Corollary 6.30. Consider $Y=Y^{\infty}(U)$ where $U$ has a predual $U^{\triangleleft}$, and suppose $A=I-K \in B D O(Y)$ is rich, has a preadjoint $A^{\triangleleft} \in L\left(Y^{\triangleleft}\right)$ where $Y^{\triangleleft}=Y^{1}\left(U^{\triangleleft}\right)$, and that $K \in U M(Y)$. Then the following statements are equivalent.

(a) all limit operators of $A$ are injective $\left(\alpha\left(A_{h}\right)=0\right.$ for all $\left.A_{h} \in \sigma^{\mathrm{op}}(A)\right)$ and there is an $S$-dense subset, $\sigma$, of $\sigma^{\mathrm{op}}(A)$ such that $\beta\left(A_{h}\right)=0$ for all $A_{h} \in \sigma$; 
(b) all limit operators of $A$ are injective $\left(\alpha\left(A_{h}\right)=0\right.$ for all $\left.A_{h} \in \sigma^{\mathrm{op}}(A)\right)$ and there is an $S$-dense subset, $\sigma$, of $\sigma^{\mathrm{op}}(A)$ such that $\alpha\left(A_{h}^{\triangleleft}\right)=0$ for all $A_{h} \in \sigma$;

(c) $A$ is invertible at infinity;

(d) A is Fredholm.

Proof. Note first that, by Lemma 6.25, each $A_{h} \in \sigma^{\mathrm{op}}(A)$ has a well-defined preadjoint $A_{h}^{\triangleleft} \in L\left(Y^{\triangleleft}\right)$ so that statement (b) is well-defined; in fact, by Lemma 6.25 . $\left\{A_{h}^{\triangleleft}: A_{h} \in \sigma^{\mathrm{op}}(A)\right\}=\sigma^{\mathrm{op}}\left(A^{\triangleleft}\right)$. Since always $\beta\left(A_{h}\right) \geq \alpha\left(A_{h}^{\triangleleft}\right)[\mathbf{3 8}$, clearly (a) $\Rightarrow(\mathrm{b})$.

If (b) holds then, noting that property (i) of Lemma 6.23 implies that $\sigma^{\mathrm{op}}(K)$ is uniformly Montel on $(Y, s)$, applying Theorem 6.3, $\sigma^{\mathrm{op}}(A)$ is uniformly bounded below, which implies that the range of each $A_{h} \in \sigma^{\mathrm{op}}(A)$ is closed. This implies that $\beta\left(A_{h}\right)=\alpha\left(A_{h}^{\triangleleft}\right)=0\left[\mathbf{3 8}\right.$, for each $A_{h} \in \sigma$, so that (b) $\Rightarrow(\mathrm{a})$ and each $A_{h} \in \sigma$ is surjective.

Applying Theorem 6.3 again, we see that all the elements of $\sigma^{\mathrm{op}}(A)$ are invertible and their inverses are uniformly bounded. Applying Theorem 6.28 we conclude that $(\mathrm{a}) \Leftrightarrow(\mathrm{b}) \Leftrightarrow(\mathrm{c})$.

The implication $(\mathrm{c}) \Rightarrow$ (d) follows from Theorem 6.28 (ii) with $C=I$ and $-K \in$ $M(Y)$ by property (iii) of Lemma 6.23 Finally, $(\mathrm{d}) \Rightarrow(\mathrm{c})$ is Theorem $6.28 \mathrm{~b}$ ).

We note that Corollary 6.30, for operators satisfying the conditions of the corollary, reduces the problem of establishing Fredholmness and/or invertibility at infinity on $Y^{\infty}(U)$ to one of establishing injectivity of the elements of $\sigma^{\mathrm{op}}(A)$ and a subset of the elements of $\sigma^{\mathrm{op}}\left(A^{\triangleleft}\right)$. In applications in mathematical physics this injectivity can sometimes be established directly via energy or other arguments (e.g. [14), this reminiscent of classical applications of boundary integral equations in mathematical physics where $A=I+K$ with $K$ compact, and injectivity of $A$ is established from equivalence with a boundary value problem.

In the one-dimensional case $N=1$ a stronger version of Theorem 5.16 can be shown, namely Theorem 6.31 below. This result is shown by establishing, in the case in which $A=I-K \in B D O(Y)$ is rich, $K \in U M(Y)$, and all the limit operators of $A$ are injective, the following three statements:

a) If $B \in \sigma^{\mathrm{op}}(A)$ has a surjective limit operator then $B$ is surjective itself.

b) Every $B \in \sigma^{\mathrm{op}}(A)$ has a self-similar limit operator.

c) Self-similar limit operators (of $A$, including those of $B$ ) are surjective.

That b) holds is Lemma 5.15. The proofs of a) and c) in [17] are both examples of the application of Theorem 5.8 .

Theorem 6.31. 17. Suppose that $Y=\ell^{\infty}(\mathbb{Z}, U)$, that $A=I-K \in B D O(Y)$ is rich, that $K \in U M(Y)$, and that all the limit operators of $A$ are injective. Then all elements of $\sigma^{\mathrm{op}}(A)$ are invertible and their inverses are uniformly bounded.

Combining this result with Corollary 6.30 we have the following simplified version of Corollary 6.30 which holds in the one-dimensional case.

Corollary 6.32. Suppose $Y=\ell^{\infty}(\mathbb{Z}, U)$ where $U$ has a predual $U^{\triangleleft}$, and suppose $A=I-K \in B D O(Y)$ is rich, has a preadjoint $A^{\triangleleft} \in L\left(Y^{\triangleleft}\right)$ where $Y^{\triangleleft}=$ $\ell^{1}\left(\mathbb{Z}, U^{\triangleleft}\right)$, and that $K \in U M(Y)$. Then the following statements are equivalent: 
(a) all limit operators of $A$ are injective;

(b) all elements of $\sigma^{\mathrm{op}}(A)$ are invertible and their inverses are uniformly bounded;

(c) A is invertible at infinity;

(d) A is Fredholm.

\subsection{Almost Periodic Band-Dominated Operators}

Recall the classes of all absolutely rich/periodic and all norm-rich/almost periodic operators as introduced in Section 6.1. We will now look at operators that are norm-rich/almost periodic and band-dominated at the same time. We first show that every norm-rich/almost periodic band-dominated operator can be approximated in the norm by norm-rich/almost periodic band operators. The same statement holds with 'norm-rich/almost periodic' replaced by 'rich', as was first pointed out in [49, Proposition 2.9]. The proof of our lemma is very similar to that of this related statement. An alternative, less constructive method for approximating a norm rich/almost periodic operator by a norm-rich/almost periodic band operator is described in step I of the proof of [42, Theorem 1].

REMARK 6.33 Note that the norm-approximation of a given band-dominated operator $A$ by a sequence $A_{n}$ of band operators is in general a more involved problem than it might seem. In particular, it is not always possible to achieve this approximation by letting $\left[A_{n}\right]$ be the restriction of $[A]$ to $n$ of its diagonals (see Remarks 1.40 and 1.44 in [51]). Instead, for a given $A \in B D O(Y)$, in the proof of [74, Theorem 2.1.6] a sequence of band operators

$$
\begin{aligned}
A_{n} & =\sum_{|k| \leq n} c_{k, n} B_{k}, \quad n \in \mathbb{N}, \\
\text { with } \quad B_{k} & =\int_{[0,2 \pi]^{N}} M_{e_{t}} A M_{e_{-t}} e^{-i(t, k)} d t, \quad k \in \mathbb{Z}^{N}
\end{aligned}
$$

is constructed, where $c_{k, n} \in \mathbb{C}$ and $e_{t}(m)=e^{i\left(t_{1} m_{1}+\ldots+t_{N} m_{N}\right)}$ for all $m \in \mathbb{Z}^{N}$ and $t \in \mathbb{R}^{N}$. This construction is such that each matrix $\left[B_{k}\right]$ is only supported on the $k$ th diagonal and $A_{n} \rightrightarrows A$ as $n \rightarrow \infty$.

Lemma 6.34. For every band-dominated operator $A$ and the corresponding approximating sequence $\left(A_{n}\right)$ of band operators (6.23), the following holds.

(i) If $A$ is norm-rich/almost periodic, then each one of the band operators $A_{n}$ is norm-rich/almost periodic.

(ii) If $A$ is rich and $\sigma^{\mathrm{op}}(A)$ is uniformly Montel, then every operator spectrum $\sigma^{\mathrm{op}}\left(A_{n}\right)$ is uniformly Montel.

Proof. Since the integrand in (6.24) is continuous in $t$, the integral can be understood in the Riemann sense and therefore $B_{k}$ can be approximated in norm by the corresponding Riemann sums

$$
R_{m}^{(k)}=\left(\frac{2 \pi}{m}\right)^{N} \sum_{j=1}^{m^{N}} M_{e_{t_{m, j}}} A M_{e_{-t_{m, j}}} e^{-i\left(t_{m, j}, k\right)}, \quad m \in \mathbb{N}
$$


as $m \rightarrow \infty$. Here $t_{m, j} \in T_{m, j}$ are arbitrary where $\left\{T_{m, j}: j=1, \ldots, m^{N}\right\}$ is a partition of $[0,2 \pi]^{N}$ into hyper-cubes of width $2 \pi / m$ (also see the proof of $[\mathbf{7 4}$, Theorem 2.1.18]).

To prove (i) it suffices, by Lemma 6.9 , to show that all Riemann sums $R_{m}^{(k)}$ are norm-rich/almost periodic. Since, as the restriction of an almost periodic (even periodic) function $\mathbb{R}^{N} \rightarrow \mathbb{C}$ to the integer grid $\mathbb{Z}^{N}$, the sequence $e_{t}$ is almost periodic for every choice of $t \in \mathbb{R}^{N}$, we get that both $M_{e_{t_{m, j}}}$ and $M_{e_{-t_{m, j}}}$ are norm-rich/almost periodic (see Lemma 6.35 below). By Lemma 6.9 again and $A \in L^{\mathrm{n} \$}(Y)$, it follows that then all of the Riemann sums $R_{m}^{(k)}$ and consequently, all operators $B_{k}$ and $A_{n}$ are norm-rich/almost periodic as well.

For the proof of (ii), let $A$ be rich and $\sigma^{\mathrm{op}}(A)$ be uniformly Montel. By Lemma 5.17 using that $A$ is rich, we get that $A \in U M(Y)$. Since every $M_{e_{t_{m}, j}}$ is rich (even norm-rich/almost periodic), we get that $M_{e_{t_{m, j}}} A M_{e_{-t_{m, j}}} \in U M(Y)$ for all $m \in \mathbb{N}$ and $j \in\left\{1, \ldots, m^{N}\right\}$, by Lemma 6.24 (c) and (d). This fact, together with formulas 6.23-6.25 and Lemma 6.24 (a), shows that all $R_{m}^{(k)}, B_{k}$ and $A_{n}$ are in $U M(Y)$. But the latter implies that $\sigma^{\mathrm{op}}\left(A_{n}\right)$ is uniformly Montel, by Lemma 5.17

Having reduced the study of norm-rich/almost periodic band-dominated operators to norm-rich/almost periodic band operators, we will now have a closer look at the latter class.

Lemma 6.35. A band operator is norm-rich/almost periodic iff all of its diagonals are almost periodic; that means

$$
A=\sum_{k \in D} M_{b_{k}} V_{k} \in L^{\mathrm{n} \$}(Y) \quad \text { iff } \quad b_{k} \in Y_{\mathrm{AP}}^{\infty}(L(U)), \quad \forall k \in D
$$

for all finite sets $D \subset \mathbb{Z}^{N}$ and $b_{k} \in Y^{\infty}(L(U)), k \in D$.

Proof. Let $D \subset \mathbb{Z}^{N}$ be finite, let $b_{k} \in Y^{\infty}(L(U))$ for all $k \in D$, and put $A=$ $\sum_{k \in D} M_{b_{k}} V_{k}$. Note that

$$
V_{-m} A V_{m}=\sum_{k \in D} M_{V_{-m} b_{k}} V_{k}
$$

for every $m \in \mathbb{Z}^{N}$. We show that a sequence of operators 6.26 converges in the operator norm iff all of the corresponding diagonals $V_{-m} b_{k}$ converge in the norm of $Y^{\infty}(L(U))$.

Suppose $A \in L^{\mathrm{n} \$}(Y)$ and take an arbitrary sequence $h=(h(n))_{n \in \mathbb{N}} \subset \mathbb{Z}^{N}$. Then there exists a subsequence $g$ of $h$ such that $V_{-g(n)} A V_{g(n)} \rightrightarrows C$ for some $C \in L(Y)$. Then, for all $i, j \in \mathbb{Z}^{N}$, with $[C]=\left[c_{i, j}\right]$ and with the restriction and extension operators $R_{i}$ and $E_{j}$ as introduced at the beginning of Section 6.3, we have that

$$
\begin{aligned}
\left\|V_{-g(n)} b_{i-j}(i)-c_{i, j}\right\|_{L(U)} & =\left\|R_{i}\left(V_{-g(n)} A V_{g(n)}-C\right) E_{j}\right\|_{L(U)} \\
& \leq\left\|V_{-g(n)} A V_{g(n)}-C\right\|_{L(Y(U))} \rightarrow 0
\end{aligned}
$$

as $n \rightarrow \infty$. Now, for every $k \in \mathbb{Z}^{N}$, define $c_{k} \in Y^{\infty}(L(U))$ by $c_{k}(i)=c_{i, i-k}$, so that $c_{k}$ is the $k$-th diagonal of $C$. From (6.27) we get that $\left\|V_{-g(n)} b_{k}-c_{k}\right\|_{Y \infty} \rightarrow 0$, so that $b_{k} \in Y_{\mathrm{AP}}^{\infty}(L(U))$ for each $k \in \mathbb{Z}^{N}$. 
Now, conversely, suppose that $b_{k} \in Y_{\mathrm{AP}}^{\infty}(L(U))$ for all $k \in D$ and take an arbitrary sequence $h=(h(n))_{n \in \mathbb{N}}$. Let $\left\{k_{1}, k_{2}, \ldots, k_{m}\right\}$ be an enumeration of $D \subset$ $\mathbb{Z}^{N}$, and choose a subsequence $h^{(1)} \subset h$ such that $V_{-h^{(1)}(n)} b_{k_{1}}$ converges. From this choose a subsequence $h^{(2)} \subset h^{(1)}$ such that also $V_{-h^{(2)}(n)} b_{k_{2}}$ converges, etc., until we arrive at a sequence $g:=h^{(m)} \subset h$ for which all $V_{-g(n)} b_{k}$ with $k \in D$ converge. Denote the respective limits by $c_{k} \in Y^{\infty}(L(U))$. Then we have $V_{-g(n)} A V_{g(n)} \rightrightarrows$ $\sum_{k \in D} M_{c_{k}} V_{k}=: C$ since

$$
\begin{aligned}
\left\|V_{-g(n)} A V_{g(n)}-C\right\|_{L(Y)} & =\left\|\sum_{k \in D}\left(M_{V_{-g(n)} b_{k}}-M_{c_{k}}\right) V_{k}\right\|_{L(Y)} \\
& \leq \sum_{k \in D}\left\|V_{-g(n)} b_{k}-c_{k}\right\|_{\infty} \rightarrow 0
\end{aligned}
$$

as $n \rightarrow \infty$, showing that $A \in L^{\mathrm{n} \$}(Y)$.

To establish the main result of this section it is convenient to introduce a further definition. For $1 \leq r \leq N$ call $A \in L(Y) r$-partially periodic if there exist $m_{1}, \ldots, m_{r} \in \mathbb{N}$ such that

$$
V_{m_{j} e^{(j)}} A=A V_{m_{j} e^{(j)}}, \quad j=1, \ldots, r .
$$

Then an $N$-partially periodic operator is precisely an absolutely rich/periodic operator. By a 0-partially periodic operator we shall mean any operator in $L(Y)$. Similarly, we shall say that $b \in Y^{\infty}(L(U))$ is $r$-partially periodic if

$$
b\left(k+m_{j} e^{(j)}\right)=b(k), \quad k \in \mathbb{Z}^{N}, j=1, \ldots, r .
$$

Then an $N$-partially periodic function is periodic. We shall say that every $b \in$ $Y^{\infty}(L(U))$ is 0-partially periodic.

Arguing as in the proof of Lemma 6.35 we can show the following result.

Lemma 6.36. A band operator is r-partially periodic iff all of its diagonals are $r$-partially periodic; that means

$$
A=\sum_{k \in D} M_{b_{k}} V_{k} \text { is r-partially periodic iff } \quad b_{k} \text { is } r \text {-partially periodic, } \quad \forall k \in D \text {, }
$$

for all finite sets $D \subset \mathbb{Z}^{N}$ and $b_{k} \in Y^{\infty}(L(U)), k \in D$.

The proof of the following theorem on the invertibility of almost periodic band operators depends in the first place on results on invertibility of periodic band operators (Theorem 6.7) and, secondly, on the possibility of approximation of almost periodic by periodic functions (cf. the study of invertibility of elliptic, almost periodic differential and pseudo-differential operators which dates back to [58, 87]). This theorem is not new; it is Theorem 1 in 42 (and see 43 ); in fact 42 provides a proof of this result for the general case $Y=Y^{p}, 1 \leq p \leq \infty$, by first reducing it to the case $p=\infty$. However, it seems of interest to include our proof which, while it has similarities with steps IV-VII in [42, Theorem 1], illustrates the application of Theorem 5.20 which carries a large part of the proof, and differs also in applying an inductive argument.

TheOREM 6.37. If $A=I+K, K \in B O\left(Y^{\infty}\right)$ is norm-rich/almost periodic, $\sigma^{\mathrm{op}}(K)$ is uniformly Montel, and $A$ is bounded below, then $A$ is invertible. 
Proof. Note first that, since $K \in \sigma^{\mathrm{op}}(K)$ if $K$ is norm rich (Theorem 6.10 (ii)), that $\sigma^{\mathrm{op}}(K)$ uniformly Montel implies $K \in M\left(Y^{\infty}\right)$.

We shall establish the theorem by proving, by induction, that, for $r=0,1, \ldots, N$,

if $A$ satisfies the conditions of the theorem and,

$$
\text { additionally, } A \text { is } r \text {-partially periodic, then } A \text { is invertible. }
$$

Statement 6.30 for $r=0$ is precisely the theorem that we wish to prove.

That 6.30 holds for $r=N$ follows from Theorem 6.7. Now suppose that 6.30 holds for $r=s$, for some $s \in\{1, \ldots, N\}$, and that $A$ satisfies the conditions of the theorem and, additionally, is $(s-1)$-partially periodic. We will show that this implies that $A$ is invertible, so that 6.30 holds for $r=s-1$, proving the inductive step. That $A$ is invertible will be proved by applying Theorem 5.20 .

First note that, by Lemmas 6.35 and 6.36 , for some finite set $D \subset \mathbb{Z}^{N}$ and $b_{k} \in Y^{\infty}(L(U)), k \in D$, it holds that

$$
K=\sum_{k \in D} M_{b_{k}} V_{k}
$$

with each $b_{k} \in Y_{\mathrm{AP}}^{\infty}(L(U))$ and $(s-1)$-partially periodic. For $n \in \mathbb{N}$ let $h(n) \in \mathbb{Z}^{N}$ have $s$ th component $n^{2}$ and all other components zero. Since each $b_{k} \in Y_{\mathrm{AP}}^{\infty}(L(U))$ we can choose a subsequence $g$ of $h$ such that $V_{-g(n)} b_{k}$ is convergent as $n \rightarrow \infty$ for each $k \in D$. Thus, defining $f(n)=g(n+1)-g(n)$,

$$
\left\|V_{-f(n)} b_{k}-b_{k}\right\|_{\infty}=\left\|V_{-g(n+1)} b_{k}-V_{-g(n)} b_{k}\right\|_{\infty} \rightarrow 0
$$

as $n \rightarrow \infty$. Note that the sth component of $f(n)$ is $\geq 2 n+1$ and that all the other components of $f(n)$ are zero.

For $j \in \mathbb{N}$ define $\hat{P}_{j} \in L\left(Y^{\infty}(L(U))\right)$ by

$$
\hat{P}_{j} b(\ell)=b(\ell), \quad-\frac{j}{2}<\ell_{s} \leq \frac{j}{2},
$$

for $\ell=\left(\ell_{1}, \ldots, \ell_{N}\right) \in \mathbb{Z}^{N}$, and by the requirement that

$$
\hat{P}_{j} b\left(\ell+j e^{(s)}\right)=\hat{P}_{j} b(\ell), \quad \ell \in \mathbb{Z}^{N} .
$$

An important observation is that, for $j \in \mathbb{N}, b \in Y^{\infty}(L(U))$,

$$
\left\|P_{j}\left(\hat{P}_{j} b-b\right)\right\|_{\infty} \leq\left\|V_{j e^{(s)}} b-b\right\|_{\infty}=\left\|V_{-j e^{(s)}} b-b\right\|_{\infty} .
$$

Using this notation, for each $k \in D$ let us define a sequence of increasingly good approximations to $b_{k}$, each approximation being $s$-partially periodic and almost periodic. In detail, for $n \in \mathbb{N}$ and $k \in D$, let

$$
b_{k}^{(n)}:=\hat{P}_{f(n)} b_{k} .
$$

Further, define $K_{n}$, an approximation to $K$, by

$$
K_{n}=\sum_{k \in D} M_{b_{k}^{(n)}} V_{k}
$$

Then it is clear that each $b_{k}^{(n)}$ is $s$-partially periodic. To see that $b_{k}^{(n)}$ is also almost periodic, note that every $\ell \in \mathbb{Z}^{N}$ can be written as $\ell=\hat{\ell}+\tilde{\ell}$, where $\hat{\ell}=\ell_{s} e^{(s)}\left(\ell_{s}\right.$ 
the $s$ th component of $\ell$ ). Then $V_{\ell}=V_{\hat{\ell}} V_{\tilde{\ell}}$ and, for $j \in \mathbb{N}, V_{\tilde{\ell}}$ commutes with $\hat{P}_{j}$. Thus, for every $n \in \mathbb{N}$ and $\ell \in \mathbb{Z}^{N}$,

$$
V_{\ell} b_{k}^{(n)}=V_{\hat{\ell}} \hat{P}_{f(n)} V_{\tilde{\ell}} b_{k}=V_{j e^{(s)}} \hat{P}_{f(n)} V_{\tilde{\ell}} b_{k},
$$

for some $j \in \mathbb{Z}$ with $|j| \leq f(n) / 2$. From this formula it is clear that $\left\{V_{\ell} b_{k}^{(n)}: \ell \in\right.$ $\left.\mathbb{Z}^{N}\right\}$ is relatively compact if $\left\{V_{\ell} b_{k}: \ell \in \mathbb{Z}^{N}\right\}$ is relatively compact, so that $b_{k}^{(n)}$ is almost periodic. Moreover it is clear that $b_{k}^{(n)} \stackrel{s}{\rightarrow} b_{k}$ as $n \rightarrow \infty$ for each $k \in D$, so that $K_{n} \stackrel{s}{\rightarrow} K$ and $A_{n}:=I+K_{n} \stackrel{s}{\rightarrow} A$. Further, since each $b_{k}^{(n)}$ is $s$-partially periodic and almost periodic, it follows from Lemmas 6.35 and 6.36 that $K_{n}$ is $s$-partially periodic and norm rich, and so therefore is $A_{n}$. Note also that, by Lemma 5.17 and the equivalence of (iii) and (v) in Lemma 6.23, the set $\left\{b_{k}(\ell): \ell \in \mathbb{Z}^{N}\right\}$ is collectively compact for every $k \in D$, so that $\left\{b_{k}^{(n)}(\ell): \ell \in \mathbb{Z}^{N}, n \in \mathbb{N}\right\} \subset\left\{b_{k}(\ell): \ell \in \mathbb{Z}^{N}\right\}$ is collectively compact. Arguing as in the proof that (v) implies (iii) in Lemma 6.23. we deduce that $\left\{V_{-j} K_{n} V_{j}: j \in \mathbb{Z}^{N}, n \in \mathbb{N}\right\}$ is uniformly Montel, so in particular, by the equivalence of (i) and (ii) in Lemma 6.23, $\sigma^{\mathrm{op}}\left(K_{n}\right)$ is uniformly Montel for each $n$. By the inductive hypothesis it follows that if $A_{n}$ is bounded below then $A_{n}$ is surjective, for each $n$.

We have shown that conditions (a)-(c) of Theorem 5.20 are satisfied by $A$ and $A_{n}$. To complete the proof we will show that conditions (d) and (e) of Theorem 5.20 are satisfied with $\mathcal{B}=\sigma^{\mathrm{op}}(A)$. Since $A$ is bounded below which implies, by (v) of Theorem 6.10 that $\mathcal{B}$ is uniformly bounded below, this choice of $\mathcal{B}$ satisfies (e) of Theorem 5.20 .

To see that (d) holds with $\mathcal{B}=\sigma^{\mathrm{op}}(A)$, suppose that $\ell(j) \in \mathbb{Z}^{N}$ and $n(j) \in \mathbb{N}$, for $j \in \mathbb{N}$, and that $n(j) \rightarrow \infty$ as $j \rightarrow \infty$, and let

$$
B_{j}:=V_{-\ell(j)} K_{n(j)} V_{\ell(j)}=\sum_{k \in D} M_{c_{k}^{(j)}} V_{k},
$$

where $c_{k}^{(j)}:=V_{-\ell(j)} b_{k}^{n(j)}$. What we have to show is that $\left(B_{j}\right)_{j \in \mathbb{N}}$ has a subsequence that $s$-converges to an element of $\sigma^{\mathrm{op}}(K)$. To see this, first note that, by 6.32 ,

$$
c_{k}^{(j)}=V_{p(j) e^{(s)}} \hat{P}_{f(n(j))} V_{-\tilde{\ell}(j)} b_{k},
$$

for some $p(j) \in \mathbb{Z}$ with $|p(j)| \leq f(n(j)) / 2$. Since each $b_{k}$ is almost periodic, setting $\check{\ell}(j):=-p(j) e^{(s)}+\tilde{\ell}(j)$, we can find a subsequence of $\check{\ell}$, which we will denote again by $\check{\ell}$, such that, for each $k \in D$, there exists $\hat{b}_{k} \in Y^{\infty}(L(U))$ such that

$$
\varepsilon_{j}:=\left\|V_{-\check{\ell}(j)} b_{k}-\hat{b}_{k}\right\|_{\infty} \rightarrow 0
$$

as $j \rightarrow \infty$. Then, for $m \in \mathbb{N}$ and all $j$ sufficiently large such that $m \leq f(n(j)) / 2$,

$$
\begin{aligned}
\left\|P_{m}\left(c_{k}^{(j)}-\hat{b}_{k}\right)\right\|_{\infty} & \leq \varepsilon_{j}+\left\|P_{m}\left(c_{k}^{(j)}-V_{-\check{\ell}(j)} b_{k}\right)\right\|_{\infty} \\
& =\varepsilon_{j}+\left\|P_{m} V_{p(j) e^{(s)}}\left(\hat{P}_{f(n(j))}-I\right) V_{-\tilde{\ell}(j)} b_{k}\right\|_{\infty} \\
& \leq \varepsilon_{j}+\left\|P_{m+|p(j)|}\left(\hat{P}_{f(n(j))}-I\right) V_{-\tilde{\ell}(j)} b_{k}\right\|_{\infty} \\
& \leq \varepsilon_{j}+\left\|P_{f(n(j))}\left(\hat{P}_{f(n(j))}-I\right) V_{-\tilde{\ell}(j)} b_{k}\right\|_{\infty} \\
& \leq \varepsilon_{j}+\left\|V_{-f(n(j))} b_{k}-b_{k}\right\|_{\infty},
\end{aligned}
$$

by 6.31. Thus $\left\|P_{m}\left(c_{k}^{(j)}-\hat{b}_{k}\right)\right\|_{\infty} \rightarrow 0$ as $j \rightarrow \infty$, for every $m$, so that $c_{k}^{(j)} \stackrel{s}{\rightarrow} \hat{b}_{k}$. 
Defining

$$
\hat{K}:=\sum_{k \in D} M_{\hat{b}_{k}} V_{k}
$$

we have that $\varepsilon_{j} \rightarrow 0$ as $j \rightarrow \infty$ for each $k \in D$, so that

$$
V_{-\check{\ell}(j)} K V_{\check{\ell}(j)}=\sum_{k \in D} M_{V_{-\check{\ell}(j)} b_{k}} V_{k} \rightrightarrows \sum_{k \in D} M_{\hat{b}_{k}} V_{k}=\hat{K} \text {. }
$$

If $\check{\ell}$ has an unbounded subsequence, this implies that $\hat{K} \in \sigma^{\mathrm{op}}(K)$. If $\check{\ell}$ does not have an unbounded subsequence then it has a constant subsequence, i.e. $\check{\ell}(j)=\tilde{\ell}$ for some $\tilde{\ell} \in \mathbb{Z}^{N}$ and infinitely many $j \in \mathbb{N}$, so that $\hat{K}=V_{-\tilde{\ell}} K V_{\tilde{\ell}}$. Since $K \in \sigma^{\mathrm{op}}(K)$ implies (by Theorem 5.12 (ii)) that $V_{-\tilde{\ell}} K V_{\tilde{\ell}} \in \sigma^{\mathrm{op}}(K)$, it follows also in this case that $\hat{K} \in \sigma^{\mathrm{op}}(K)$. But $c_{k}^{(j)} \stackrel{s}{\rightarrow} \hat{b}_{k}$ for every $k \in D$ implies that

$$
B_{j}=\sum_{k \in D} M_{c_{k}^{(j)}} V_{k} \stackrel{s}{\rightarrow} \sum_{k \in D} M_{\hat{b}_{k}} V_{k}=\hat{K},
$$

so that we have shown that $\left(B_{j}\right)_{j \in \mathbb{N}}$ has a subsequence that $s$-converges to an element of $\sigma^{\mathrm{op}}(K)$.

Now we can finally prove the generalisation of Corollary 6.8 to norm-rich/almost periodic band-dominated operators. We note that results of this flavour in concrete cases, in particular showing something close to equivalence of (i) and (iii), date back at least to Shubin 87. for scalar elliptic differential operators with smooth almost periodic coefficients, where the analogous statement to (iii) is termed the Favard condition (and see $\mathbf{1 7}$ ). Note also that we have already seen, in Theorem 6.31 that in the one-dimensional case $N=1$ the equivalence of (iii) and (iv) holds even when $K$ is only rich rather than norm rich/almost periodic.

THEOREM 6.38. If $A=I+K$ with $K \in B D O\left(Y^{\infty}\right)$ norm-rich/almost periodic and $\sigma^{\mathrm{op}}(K)$ is uniformly Montel, then the following statements are equivalent.

(i) $A$ is invertible;

(ii) $A$ is bounded below;

(iii) all limit operators of $A$ are injective;

(iv) all limit operators of $A$ are invertible with uniformly bounded inverses.

Proof. Let the conditions of the theorem be fulfilled. Implication (iv) $\Rightarrow$ (i) follows by Theorem 6.10 (ii); (i) $\Rightarrow$ (ii) is trivial; (ii) $\Rightarrow$ (iii) follows by Theorem 6.10 (v); and it remains to show (iii) $\Rightarrow(\mathrm{iv})$.

Suppose (iii) holds. By Theorem 6.3 it follows that $\sigma^{\mathrm{op}}(A)$ is uniformly bounded below, so that, by Theorem $6.10(\mathrm{v}), \nu\left(A_{h}\right)=\nu(A)>0$ for all $A_{h} \in \sigma^{\mathrm{op}}(A)$. Now, from Lemma 6.34 applied to $K$ in place of $A$, we know that there exists a sequence $\left(K_{n}\right)$ of norm-rich/almost periodic band operators with $\sigma^{\mathrm{op}}\left(K_{n}\right)$ uniformly Montel for each $n$ and $K_{n} \rightrightarrows K$. From (5.6) it follows that $\nu\left(I+K_{n}\right) \rightarrow \nu(I+K)=$ $\nu(A)>0$, so that there exists a $n_{0} \in \mathbb{N}$ with

$$
\nu\left(I+K_{n}\right)>\frac{1}{2} \nu(A)>0, \quad n \geq n_{0} .
$$

Invoking Theorem 6.37, we conclude that, for every $n \geq n_{0}, I+K_{n}$ is invertible and

$$
\left\|\left(I+K_{n}\right)^{-1}\right\|=\frac{1}{\nu\left(I+K_{n}\right)}<\frac{2}{\nu(A)}<\infty .
$$


Since also $I+K_{n} \rightrightarrows I+K=A$, we get, by [51, Lemma 1.3], that $A$ is invertible. From Theorem 6.10 (iv) and (v) it now follows that each limit operator $A_{h}$ of $A$ is invertible and

But this is (iv).

$$
\left\|\left(A_{h}\right)^{-1}\right\|=\frac{1}{\nu\left(A_{h}\right)}=\frac{1}{\nu(A)}<\infty .
$$

\subsection{The Wiener Algebra}

If we write of "all spaces $Y$ " in this section, we always think of the set of spaces $Y=Y^{p}(U)$ where $U$ is fixed, and only $p$ varies in $\{0\} \cup[1, \infty]$. For a band operator $A$ of the form 6.15 , , one clearly has

$$
\|A\|_{L(Y)}=\left\|\sum_{|k| \leq w} M_{b_{k}} V_{k}\right\|_{L(Y)} \leq \sum_{k \in \mathbb{Z}^{N}}\left\|b_{k}\right\|_{Y^{\infty}}=:\|A\|_{\mathcal{W}}
$$

for all spaces $Y$, where we put $b_{k}=0$ if $|k|>w$. By $\mathcal{W}=\mathcal{W}(U)$ we denote the closure of $B O(Y)$ with respect to $\|\cdot\|_{\mathcal{W}}$. Equipped with $\|\cdot\|_{\mathcal{W}}$, the set $\mathcal{W}$ is a Banach algebra. Clearly $\mathcal{W}$ does not depend on the underlying space $Y$ and we have, for all spaces $Y$,

$$
B O(Y) \subset \mathcal{W} \subset B D O(Y) .
$$

Both inclusions are proper as the operators $A$ and $B$ with $(A x)(k)=x(-k) /|k|^{2}$ and $(B x)(k)=x(-k) /|k|$ if $k \neq 0,(A x)(0)=0=(B x)(0)$ show.

$\mathcal{W}$ is referred to as the Wiener algebra. Note that this is a natural (nonstationary) extension of the classical algebra of all matrix operators with constant diagonals and $\|A\|_{W}<\infty$ (which is isomorphic, via Fourier transform, to the algebra of all periodic functions with absolutely summable sequence of Fourier coefficients that is usually associated with the name 'Wiener algebra'). As a consequence of the definition of $\mathcal{W}$ we get that

$$
\|A\|_{L(Y)} \leq\|A\|_{\mathcal{W}}
$$

for all $A \in \mathcal{W}$ and all spaces $Y$. For operators in the Wiener algebra $\mathcal{W}$ one has the following remarkable results (see Theorem 2.5.2 and Proposition 2.5.6 of [74]).

Lemma 6.39. For every $A \in \mathcal{W}$, the following holds.

(i) If $A$ is invertible then $A^{-1} \in \mathcal{W}$, so that $\mathcal{W}$ is inverse closed.

(ii) If $A$ is rich and $h=(h(n)) \subset \mathbb{Z}^{N}$ tends to infinity then there is a subsequence $g$ of $h$ such that the limit operator $A_{g}$ exists with respect to all spaces $Y$. This limit operator again belongs to $\mathcal{W}$, and $\left\|A_{g}\right\|_{\mathcal{W}} \leq\|A\|_{\mathcal{W}}$.

As a consequence of Lemma 6.39 we get that, if $A \in \mathcal{W}$ is invertible on one of the spaces $Y$ then it is invertible on all spaces $Y$, and $\left\|A^{-1}\right\|_{L(Y)} \leq\left\|A^{-1}\right\|_{\mathcal{W}}$. Another consequence of this lemma is that the operator spectrum $\sigma^{\mathrm{op}}(A)$ is contained in the Wiener algebra $\mathcal{W}$ and does not depend on the underlying space $Y$ if $A \in \mathcal{W}$. So for $A \in \mathcal{W}$, the statement of Theorem 6.28 (iii) holds independently of the underlying space $Y$. Moreover, by Theorem 6.28 (iv), also the uniform boundedness condition of the inverses is redundant since this is true for $p \in\{0,1, \infty\}$ and consequently, also for $p \in(1, \infty)$ by Riesz-Thorin interpolation: 
TheOrEm 6.40. If $A \in \mathcal{W}$ is rich then the following statements are equivalent.

(i) A is invertible at infinity on one of the spaces $Y$.

(ii) $A$ is invertible at infinity on all the spaces $Y$.

(iii) All limit operators of $A$ are invertible on one of the spaces $Y$.

(iv) All limit operators of $A$ are invertible on all the spaces $Y$ and

$$
\sup _{p \in\{0\} \cup[1, \infty]} \sup _{A_{h} \in \sigma^{\mathrm{op}}(A)}\left\|A_{h}^{-1}\right\|_{L\left(Y^{p}\right)}<\infty .
$$

REMARK 6.41 This theorem is a significant strengthening and simplification of Theorem 2.5.7 in [74. Theorem 2.5.7 requires that $U$ is reflexive, and, in the case that $U$ is reflexive, it implies only a reduced version of our Theorem 6.40 with the value of $Y$ restricted to $Y^{p}, p \in\{0\} \cup(1, \infty)$, in (i)-(iii).

Proof of Theorem 6.40, (i) $\Rightarrow$ (iii) follows from Theorem 6.28 (iii).

(iii) $\Rightarrow$ (iv): Suppose (iii) holds. We have observed already that $\sigma^{\mathrm{op}}(A) \subset \mathcal{W}$ is independent of the space $Y$ by Lemma 6.39 (ii). Applying Lemma 6.39 (i) to the limit operators of $A$, it follows that these limit operators are invertible on all the spaces $Y$. By Theorem 6.28 (iv),

$$
s_{p}:=\sup _{A_{h} \in \sigma^{\circ \mathrm{p}}(A)}\left\|A_{h}^{-1}\right\|_{L\left(Y^{p}\right)}
$$

is finite for $p \in\{0,1, \infty\}$. Now, by Riesz-Thorin interpolation (as demonstrated in the proof of [74, Theorem 2.5.7]), we get that $s_{p} \leq s_{1}^{1 / p} s_{\infty}^{1-1 / p}<\infty$ for all $p \in(1, \infty)$, which proves (iv).

(iv) $\Rightarrow$ (ii) follows from Theorem 6.28 (iii).

Finally, (ii) $\Rightarrow($ i) is evident.

From the above result and the relationship between invertibility at infinity and Fredholmness, (see Remark 6.2 and Theorem 6.28 a)(i)+(ii) and b)), we can deduce Corollary 6.43 below, which relates Fredholmness to invertibility of limit operators. In this corollary we require, for the equivalence of (a)-(d) with (e), the existence of a predual $U^{\triangleleft}$ and, for $A$ considered as an operator on $Y^{\infty}$, the existence of a preadjoint $A^{\triangleleft} \in Y^{1}\left(U^{\triangleleft}\right)$. The following obvious lemma characterises existence of a preadjoint in terms of existence of preadjoints of the elements of the matrix representation of $A$.

Lemma 6.42. If $A \in \mathcal{W}(U)$, with $[A]=\left[a_{m n}\right]$, and $U$ has a predual $U^{\triangleleft}$, then $A$, considered as an operator on $Y^{\infty}(U)$, has a preadjoint $A^{\triangleleft} \in Y^{1}\left(U^{\triangleleft}\right)$ iff each entry $a_{m n} \in L(U)$ of the matrix representation of $A$ has a preadjoint $a_{m n}^{\triangleleft} \in L\left(U^{\triangleleft}\right)$. If this latter condition holds then a preadjoint is $A^{\triangleleft} \in \mathcal{W}\left(U^{\triangleleft}\right)$ with $\left[A^{\triangleleft}\right]=\left[a_{n m}^{\triangleleft}\right]$. In particular, $A$ has a preadjoint if $U$ is reflexive, given by $A^{\triangleleft} \in \mathcal{W}\left(U^{*}\right)=\mathcal{W}\left(U^{\triangleleft}\right)$, with $\left[A^{\triangleleft}\right]=\left[a_{n m}^{*}\right]$, where $a_{n m}^{*} \in L\left(U^{*}\right)=L\left(U^{\triangleleft}\right)$ is the adjoint of $a_{n m}$.

Corollary 6.43. Suppose $A=I-K \in \mathcal{W}$ is rich, and $K \in U M(Y)$. Then the following statements are equivalent.

(a) All limit operators of $A$ are injective on $Y^{\infty}$ and $\sigma^{\mathrm{op}}(A)$ has an $S$-dense subset, $\sigma$, such that $\beta\left(A_{h}\right)=0$ on $Y^{\infty}$ for all $A_{h} \in \sigma$;

(b) $A$ is invertible at infinity on all the spaces $Y$;

(c) $A$ is invertible at infinity on one of the spaces $Y$; 
(d) A is Fredholm on all the spaces $Y$.

In the case that $U$ has a predual $U^{\triangleleft}$ and $A$, considered as an operator on $Y^{\infty}(U)$, has a preadjoint $A^{\triangleleft} \in Y^{1}\left(U^{\triangleleft}\right)$, then (a)-(d) are equivalent to

(e) $A$ is Fredholm on one of the spaces $Y$.

Proof. For the clarity of our argument we introduce two more statements:

(f) All limit operators of $A$ are invertible on $Y^{\infty}$;

(g) $A$ is invertible at infinity on $Y^{2}$.

Each of these will turn out to be equivalent to (a)-(d).

By Theorem 6.3 statement (a) is equivalent to (f), which, by Theorem 6.40, is equivalent to each of (b), (c) and (g).

Since $K \in M(Y)$, the implication (b) $\Rightarrow(\mathrm{d})$ follows from Theorem 6.28 (ii) (applied with $C=-I$ ).

Since, obviously, (d) implies Fredholmness of $A$ on $Y^{2}$, it also implies (g), by Theorem 6.28 (i). Another obvious consequence of (d) is (e).

Finally, suppose $U^{\triangleleft}$ and $A^{\triangleleft}$ exist and (e) holds for $Y=Y^{p}$. If $p=\infty$, then (c) follows by Theorem $6.28 \mathrm{~b}$ ), and otherwise, if $p<\infty$, then (c) follows by Theorem 6.28 (i).

The above corollary implies, for rich operators in the Wiener algebra which are of the form $A=I-K \in \mathcal{W}$ with $K \in U M(Y)$, i.e. $K$ is subject to the (equivalent) properties (i)-(v) in Lemma 6.23, and which possess a preadjoint, that Fredholmness on one of the spaces $Y$ implies Fredholmness on all spaces $Y$. The argument to show this is indirect: it depends on the connection between Fredholmness and invertibility at infinity and on the equivalence of (i) and (ii) in Theorem 6.40 .

Recently, Lindner $5 \mathbf{5 2}$ has studied directly the invariance of the Fredholm property across the spaces $Y$ for general operators in the Wiener algebra, but with a slight restriction on the Banach space $U$, that it is either finite-dimensional or possesses the hyperplane property, meaning that it is isomorphic to a subspace of $U$ of co-dimension 1. An equivalent characterisation of the hyperplane property 52 is that there exists a $B \in L(U)$ which is Fredholm of index 1. This characterisation suggests that infinite-dimensional Banach spaces which do not have the hyperplane property are unusual. In $\mathbf{5 3}$. Lindner lists many sets of conditions on $U$ which ensure that $U$ has the hyperplane property, and recalls that it was a long-standing open problem due to Banach - the so-called hyperplane problem - whether there exist any infinite-dimensional Banach spaces which do not have the hyperplane property. An example was finally constructed by Gowers in 35, for which work, and the resolution of other long-standing open questions posed by Banach, he received the Fields medal in 1998.

The main result proved by Lindner $\mathbf{5 2}$ is the following:

Theorem 6.44. Suppose that $U$ is finite-dimensional or has the hyperplane property and that $A \in \mathcal{W}(U)$. Then:

(a) If $A$ is Fredholm on one of the spaces $Y^{p}$, with $p \in\{0\} \cup[1, \infty)$, then $A$ is Fredholm on all spaces $Y$. 
(b) If $U$ has a predual $U^{\triangleleft}$ and $A$, considered as acting on $Y^{\infty}$, has a preadjoint $A^{\triangleleft} \in Y^{1}\left(U^{\triangleleft}\right)$, then $A$ is Fredholm on one of the spaces $Y$ iff $A$ is Fredholm on all of the spaces $Y$.

If $A$ is Fredholm on every space $Y$ then the index is the same on each space.

By combining Theorem 6.40 and Corollary 6.43 we get the following result. In this result, again, by $\operatorname{spec}_{\text {ess }}(A)$ we denote the essential spectrum of $A$ (the set of $\lambda$ for which $\lambda I-A$ is not Fredholm) and by $\operatorname{spec}(A)$ the ordinary spectrum of $A$.

Corollary 6.45. Suppose $A=I-K \in \mathcal{W}$ is rich, $K \in U M(Y)$, $U$ has a predual $U^{\triangleleft}$, and that $A$, considered as an operator on $Y^{\infty}(U)$, has a preadjoint $A^{\triangleleft} \in Y^{1}\left(U^{\triangleleft}\right)$. Then statements (i)-(iv) of Theorem 6.40 and (a)-(e) of Corollary 6.43 are all equivalent. Further, on every space $Y$ it holds that

$$
\operatorname{spec}_{\mathrm{ess}}(A)=\bigcup_{B \in \sigma^{\mathrm{op}}(A)} \operatorname{spec}(B) .
$$

Proof. It remains only to show that, for every $\lambda \in \mathbb{C}, \lambda I-A=(\lambda-1) I+K$ is Fredholm iff $(\lambda-1) I+L$ is invertible for every $L \in \sigma^{\mathrm{op}}(K)$. For $\lambda \neq 1$ this follows from the earlier part of the corollary. This is true also for $\lambda=1$ when $U$ is finite-dimensional (see Corollary 6.46 below). When $U$ is infinite-dimensional and $\lambda=1$, then $(\lambda-1) I+K=K \in U M(Y) \subset M(Y)$ and so $(\lambda-1) I+L=L \in M(Y)$. This implies that all the entries of the matrix representations of $(\lambda-1) I+K$ and $(\lambda-1) I+L$ are compact (in $K(U)$ ). Since $U$ is infinite-dimensional, this implies that $(\lambda-1) I+K$ and $(\lambda-1) I+L$ are not Fredholm.

In the particularly simple case of a finite-dimensional space $U$ we have the following extended version of Corollary 6.45.

Corollary 6.46. Suppose $A \in \mathcal{W}$ and $U$ is finite-dimensional. Then statements (i)-(iv) of Theorem 6.40 and (a)-(e) of Corollary 6.43 are all equivalent. Moreover, if $A$ is subject to all these equivalent statements then the index of $A$ is the same on each space $Y$. Further, on every space $Y$, 6.35) holds.

Proof. To see that the conditions of Corollary 6.45 are fulfilled, recall Lemma 6.21 and remember that, by Lemma 6.24 (b), $K=I-A \in U M(Y)$ if $\operatorname{dim} U<\infty$. Also recall that finite-dimensional spaces $U$ are reflexive, so that $U^{\triangleleft}=U^{*}$ is a predual and existence of a preadjoint follows from Lemma 6.42.

It remains to show that the index of $A$ is the same on all spaces $Y$. This follows from Theorem 6.44 above. More directly, let $A$ be Fredholm on one of the spaces $Y=Y^{p}$ with $p \in\{0\} \cup[1, \infty]$ and denote its index by $\operatorname{ind}_{p} A$. From the equivalence of (d) and (e) in Corollary 6.43 we get that $A$ is Fredholm on $Y^{2}$. Now proceed exactly as in the proof of $\mathbf{7 8}$, Lemma 2.1] to arrive at $\operatorname{ind}_{p} A=\operatorname{ind}_{2} A$ (see the remark below for some details).

REMARK 6.47 a) In [78, Lemma 2.1] the independence of the index just follows for the spaces $Y^{p}$ with $p \in\{0\} \cup(1, \infty)$ because this is the setting for which [72, Theorem 8] and [74, Theorem 2.5.7] yield that the Fredholm property is independent of the underlying space. Thanks to the equivalence of (d) and (e) in Corollary 6.43, as we proved earlier in Corollary 6.45 (and 6.46), we can now enlarge this framework to $p \in\{0\} \cup[1, \infty]$. 
b) For simplicity, [78, Lemma 2.1] was only stated for band operators but its proof actually applies to all operators $A \in \mathcal{W}$. We make use of this fact in the proof of our Corollary 6.46 .

c) Also, for simplicity, [78, Lemma 2.1] was only stated for operators on $Y^{p}(\mathbb{C})$ instead of $Y^{p}(U)$ with $n:=\operatorname{dim} U<\infty$. This is not a loss of generality since these two spaces are isomorphic and the discussed operator properties are preserved under this isomorphism. Indeed, fix a basis in $U$ and let $\varphi: U \rightarrow \mathbb{C}^{n}$ refer to the isomorphism that maps $u \in U$ to its coordinate vector $\varphi(u)=:\left(\varphi_{1}(u), \ldots, \varphi_{n}(u)\right) \in$ $\mathbb{C}^{n}$ with respect to this basis. Then $\Phi: Y^{p}(U) \rightarrow Y^{p}(\mathbb{C})$ with

$$
\begin{aligned}
(\Phi x)\left(n \cdot m_{1}+k, m_{2}, \ldots, m_{N}\right)= & \varphi_{k}\left(x\left(m_{1}, \ldots, m_{N}\right)\right) \in \mathbb{C}, \\
& k \in\{1, \ldots, n\}, m_{1}, \ldots, m_{N} \in \mathbb{Z}
\end{aligned}
$$

for every $x \in Y^{p}(U)$ is an isomorphism. On the operator side, roughly speaking, the matrix representation of an operator on $Y^{p}(U)$ is an infinite matrix the entries of which are $n \times n$ matrices (operators on $U \cong \mathbb{C}^{n}$, via $\varphi$ ). Via $\Phi$ this matrix is identified, in a natural way, with an infinite matrix with scalar entries, and this is the setting in which [78, Lemma 2.1] applies. Note that this identification preserves membership of the Wiener algebra, Fredholmness, and the index of the operator.

Finally, we note that in the one-dimensional case $N=1$ we have the following refinement of Corollaries 6.43, 6.45 and 6.46, as a consequence of Theorem 6.31

Corollary 6.48. Suppose $N=1$ and that $A=I-K \in \mathcal{W}$ is rich and $K \in U M(Y)$. Then the following statements are equivalent:

(a) All limit operators of $A$ are injective on $Y^{\infty}$;

(b) All limit operators of $A$ are invertible on one of the spaces $Y$;

(c) All limit operators of $A$ are invertible on all the spaces $Y$ and (6.34) holds;

(d) $A$ is invertible at infinity on all the spaces $Y$;

(e) $A$ is invertible at infinity on one of the spaces $Y$;

(f) $A$ is Fredholm on all the spaces $Y$.

In the case that $U$ has a predual $U^{\triangleleft}$ and $A$, considered as an operator on $Y^{\infty}(U)=\ell^{\infty}(\mathbb{Z}, U)$, has a preadjoint $A^{\triangleleft}$ on $Y^{1}\left(U^{\triangleleft}\right)=\ell^{1}\left(\mathbb{Z}, U^{\triangleleft}\right)$, then $(a)-(f)$ are equivalent to

(g) A is Fredholm on one of the spaces $Y$;

and on every space $Y$ it holds that

$$
\operatorname{spec}_{\text {ess }}(A)=\bigcup_{B \in \sigma^{\text {op }}(A)} \operatorname{spec}(B)=\bigcup_{B \in \sigma^{\text {op }}(A)} \operatorname{spec}_{\text {point }}^{\infty}(B) .
$$

Here we denote by $\operatorname{spec}_{\text {point }}^{\infty}(B)$ the point spectrum (set of eigenvalues) of $B$, considered as an operator on $Y^{\infty}$.

Corollary 6.49. Suppose $N=1, A \in \mathcal{W}$ and $U$ is finite-dimensional. Then statements (a)- $(g)$ of Corollary 6.48 are equivalent. Moreover, if $A$ is subject to all these equivalent statements then the index of $A$ is the same on each space $Y$. Further, on every space $Y$, (6.36) holds. 


\section{CHAPTER 7}

\section{Discrete Schrödinger Operators}

In this chapter we illustrate the results of Chapter 6 in particular the results of Section 6.5, in the relatively simple but practically relevant setting of $Y=$ $Y^{p}=Y^{p}(U)=\ell^{p}\left(\mathbb{Z}^{N}, U\right)$ with $p \in\{0\} \cup[1, \infty]$ and a finite-dimensional space $U$. For applications to a class of operators on $Y^{p}(U)$ with $U$ infinite-dimensional, see Chapter 8 .

In this chapter we suppose that our operator $A$ is a discrete Schrödinger operator on $Y$ in the sense e.g. of [27. By this we mean that $A$ is of the form

$$
A=L+M_{b}
$$

with a translation invariant operator $L$, i.e. $V_{-\alpha} L V_{\alpha}=L$ for all $\alpha \in \mathbb{Z}^{N}$, and with a multiplication operator $M_{b}$, given by (4.4), with $b \in Y^{\infty}(L(U))$. A translation invariant operator $L$ on $Y$ is often referred to as a Laurent operator, and the sequence $b$ is typically called the potential of $A$. The matrix representation of $L$ is a Laurent matrix $[L]=\left[\lambda_{i-j}\right]_{i, j \in \mathbb{Z}^{N}}$ with $\lambda_{k} \in L(U)$ for all $k \in \mathbb{Z}^{N}$. To be able to apply the results of the previous subsections we will suppose that $A=L+M_{b} \in L\left(Y^{p}\right)$, for $1 \leq p \leq \infty$, which is the case if $L \in \mathcal{W}$, i.e. if

$$
\|L\|_{\mathcal{W}}=\sum_{k \in \mathbb{Z}^{N}}\left\|\lambda_{k}\right\|<\infty .
$$

Discrete (or lattice) Schrödinger operators are widely studied in mathematical physics (see e.g. [76, §XI.14], 83, [11, [40, 89, [27, [47, 8], 69]). Particularly common and classical is the case where the Laurent operator takes the form

$$
L=\sum_{k=1}^{N}\left(V_{e^{(k)}}+V_{-e^{(k)}}\right),
$$

where $e^{(1)}, \ldots, e^{(N)}$ are the unit coordinate vectors in $\mathbb{Z}^{N}$. The operator $A=L+M_{b}$ is then a discrete analogue of the second order differential operator $-\Delta+M$ where $\Delta$ is the Laplacian and $M$ is the operator of multiplication by a bounded potential, both on $\mathbb{R}^{N}$.

Let $L^{\prime}$ be the Laurent operator with matrix representation $\left[L^{\prime}\right]=\left[\lambda_{j-i}^{*}\right]_{i, j \in \mathbb{Z}^{N}}$. Then, identifying $U$ with $U^{*}$ (so that $U=U^{*}=U^{\triangleleft}$ ), $L^{\prime} \in \mathcal{W}$ and $A^{\prime}:=L^{\prime}+M_{b} \in$ $\mathcal{W}$. Further, in the case $p=\infty$, when we consider $A$ as an operator on $Y=Y^{\infty}$, $A^{\prime}$, considered as an operator on $Y^{1}$, is the unique transpose of $A$ with respect to the dual system $\left(Y^{\infty}, Y^{1}\right)$ of Section 6.2 and so the unique preadjoint of $A$, for it holds for $x \in Y, y \in Y^{1}$, using equations 6.9, , 6.8), and (6.14), that

$$
A x(y)=(A x, y)=\left(x, A^{\prime} y\right)=x\left(A^{\prime} y\right) .
$$


We will say that $A$ is symmetric if $A=A^{\prime}$. For example, this is the case when $L$ is the classical operator 7.1).

For $b \in Y^{\infty}(L(U))$ let Lim $(b)$ denote the set of limit functions of $b$, by which we mean the set of all functions $b_{h} \in Y^{\infty}(L(U))$ for which there exists a sequence $h: \mathbb{N} \rightarrow \mathbb{Z}^{N}$ tending to infinity such that

$$
b_{h}(m)=\lim _{n \rightarrow \infty} b(m+h(n)), \quad m \in \mathbb{Z}^{N} .
$$

It follows from 4.5 that

$$
\sigma^{\mathrm{op}}(A)=\left\{L+M_{c}: c \in \operatorname{Lim}(b)\right\} .
$$

Noting that Corollary 6.46 applies to $A=L+M_{b}$, we have the following result.

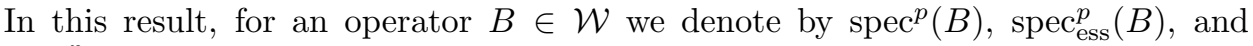
$\operatorname{spec}_{\text {point }}^{p}(B)$, respectively, the spectrum, essential spectrum, and point spectrum (set of eigenvalues) of $B$ considered as an operator on $Y^{p}$.

THEOREM 7.1. The following statements are equivalent:

(a) $L+M_{c}$ is injective on $Y^{\infty}$ for all $c \in \operatorname{Lim}(b)$ and, for some s-dense subset, $\varsigma$, of $\operatorname{Lim}(b), L^{\prime}+M_{c}$ is injective on $Y^{1}$ for all $c \in \varsigma$.

(b) $L+M_{c}$ is invertible, for every $c \in \operatorname{Lim}(b)$, on one of the spaces $Y^{p}$;

(c) $L+M_{c}$ is invertible on $Y^{p}$ for every $p$ and every $c \in \operatorname{Lim}(b)$ and the inverses are uniformly bounded (in $p$ and $c$ );

(d) $A$ is Fredholm on one of the spaces $Y^{p}$;

(e) $A$ is Fredholm on all of the spaces $Y^{p}$ and the index is the same on each space.

Thus, for every $p$ it holds that

$$
\begin{aligned}
\operatorname{spec}_{\mathrm{ess}}^{p}(A) & =\bigcup_{c \in \operatorname{Lim}(b)} \operatorname{spec}^{p}\left(L+M_{c}\right) \\
& =\bigcup_{c \in \operatorname{Lim}(b)}\left[\operatorname{spec}_{\text {point }}^{\infty}\left(L+M_{c}\right) \cup \operatorname{spec}_{\text {point }}^{1}\left(L^{\prime}+M_{c}\right)\right]
\end{aligned}
$$

and

$$
\begin{aligned}
\operatorname{spec}^{p}(A) & =\operatorname{spec}_{\text {point }}^{\infty}(A) \cup \operatorname{spec}_{\text {point }}^{1}\left(A^{\prime}\right) \cup \operatorname{spec}_{\text {ess }}^{p}(A) \\
& =\operatorname{spec}_{\text {point }}^{1}(A) \cup \operatorname{spec}_{\text {point }}^{1}\left(A^{\prime}\right) \cup \operatorname{spec}_{\text {ess }}^{p}(A) .
\end{aligned}
$$

Proof. From the equivalence of (a) and (b) in Corollary 6.30 we have that (a) is equivalent to the statement that $\alpha\left(L+M_{c}\right)=0$ for all $c \in \operatorname{Lim}(b)$ and $\beta\left(L+M_{c}\right)=0$ for all $c \in \varsigma$. By Corollary 6.46 this is equivalent to (b)-(e). So it remains to prove (7.5) +7.6$)$. Equality (7.5) follows since, as noted after Lemma 6.39, the spectrum of $A$ does not depend on $p$, so that $\operatorname{spec}^{p}(A)=\operatorname{spec}^{\infty}(A)$. Further, if $\lambda \in \operatorname{spec}^{\infty}(A)$ and $\lambda I-A$ is Fredholm, then either $\alpha(\lambda I-A) \neq 0$ or $\beta(\lambda I-A) \neq 0$, so that either $\lambda \in \operatorname{spec}_{\text {point }}^{\infty}(A)$ or $\lambda \in \operatorname{spec}_{\text {point }}^{1}\left(A^{\prime}\right)$. To see equality 7.6 , note that $\operatorname{spec}_{\text {point }}^{1}(A) \subset \operatorname{spec}_{\text {point }}^{\infty}(A)$ since injectivity of $\lambda I-A$ on $Y^{\infty}$ implies its injectivity on $Y^{1} \subset Y^{\infty}$. Moreover, if $\lambda I-A$ is Fredholm then, by Theorem 6.17, the kernel of $\lambda I-A$ is a subset of $Y^{0}$. Since $\left.(\lambda I-A)\right|_{Y^{0}}$ is Fredholm with the same index on $Y^{0}$ and $Y^{1} \subset Y^{0}$ and since $Y^{1}$ is dense in $Y^{0}$, it follows from a standard result on Fredholm operators (e.g. [66] ) that the kernel of $\lambda I-A$ is a subset of $Y^{1}$. Thus $\operatorname{spec}_{\text {point }}^{\infty}(A) \subset \operatorname{spec}_{\text {ess }}^{\infty}(A) \cup \operatorname{spec}_{\text {point }}^{1}(A)$. 
REMARK 7.2 We note that main parts of the above result, namely equality (7.3) and that the spectrum and essential spectrum do not depend on $p \in[1, \infty]$, are well known (see e.g. [74, Theorem 5.8.1]). The characterisation of the essential spectrum by (7.4) appears to be new.

Clearly, equations $7.3-7.6$ simplify when $L$ is symmetric, for example if $L$ is given by 7.1 , since we then have that $\operatorname{spec}_{\text {point }}^{1}\left(A^{\prime}\right)=\operatorname{spec}_{\text {point }}^{1}(A)$ and $\operatorname{spec}_{\text {point }}^{1}\left(L^{\prime}+M_{c}\right) \subset \operatorname{spec}_{\text {point }}^{\infty}\left(L+M_{c}\right)$ for all $c \in \operatorname{Lim}(b)$. Simplifications also occur when the potential $b$ is almost periodic, $b \in Y_{\mathrm{AP}}^{\infty}(L(U))$, in which case $\operatorname{Lim}(b)$ is precisely what is often called the hull of $b$, the set $\operatorname{clos}_{Y \infty(L(U))}\left\{V_{k} b: k \in \mathbb{Z}^{N}\right\}$, the closure of the set of translates of $b$.

THEOREM 7.3. If $b$ is almost periodic then, for all $p$ and all $\tilde{b} \in \operatorname{Lim}(b)$,

$$
\operatorname{spec}_{\mathrm{ess}}^{p}(A)=\operatorname{spec}^{p}(A)=\operatorname{spec}^{p}\left(L+M_{\tilde{b}}\right)=\bigcup_{c \in \operatorname{Lim}(b)} \operatorname{spec}_{\text {point }}^{\infty}\left(L+M_{c}\right) .
$$

Proof. $L$ is absolutely rich/periodic and so norm rich. Since $b$ is almost periodic, $M_{b}$ is norm rich by Lemma 6.35. Thus $A$ is norm rich. Further, $\sigma^{\mathrm{op}}(A-I)$ is uniformly bounded by Theorem 5.12 (i) and so uniformly Montel on $Y^{\infty}$ by Corollary 3.25 . since $\operatorname{dim} U<\infty$. The result thus follows from Theorem 6.38. Theorem 6.10 (iv), and the equivalence of statements (b) and (d) in Theorem 7.1.

Remark 7.4 That $\operatorname{spec}_{\mathrm{ess}}^{p}(A)=\operatorname{spec}^{p}(A)=\operatorname{spec}^{p}\left(L+M_{\tilde{b}}\right)$ for all $\tilde{b} \in \operatorname{Lim}(b)$, the hull of $b$, is a classical result, see e.g. $8 \mathbf{8 3}, 89,674$. The result that $\operatorname{spec}^{p}(A)=$ $\bigcup_{c \in \operatorname{Lim}(b)} \operatorname{spec}_{\text {point }}^{\infty}\left(L+M_{c}\right)$ appears to be new in this discrete setting, although analogous results for uniformly elliptic differential operators on $\mathbb{R}^{N}$ with almost periodic coefficients date back to Shubin 87 .

Moreover, note that this result is well-known, as a part of Floquet-Bloch theory 40, 41, 28, in the case when $b$ is periodic; in fact one has the stronger result in that case, at least when $L$ is given by (7.1), that $\lambda$ is in the spectrum of $A$ iff there exists a solution $x \in Y^{\infty}(U)$ of $\lambda x=A x$ which is quasi-periodic in the sense of 40 . The latter means that $x(m)=\exp (i k \cdot m) y(m)$ for all $m \in \mathbb{Z}^{N}$, where $y \in Y^{\infty}(U)$ is periodic and $k \in \mathbb{R}^{N}$ is fixed, so that if $x$ is quasi-periodic then it is certainly almost periodic. Thus, if $b$ is periodic then $\lambda$ is in the spectrum of $A$ iff there exists a solution $x \in Y^{\infty}(U)$ of $\lambda x=A x$ which is almost periodic.

Natural questions are whether this statement still holds for the case when $b$ is almost periodic, at least for $L$ given by (7.1), or whether the weaker statement holds that $\lambda$ is in the spectrum of $A$ iff, for some $c \in \operatorname{Lim}(b)$, there exists a solution $x \in Y^{\infty}(U)$ of $\lambda x=\left(L+M_{c}\right) x$ which is almost periodic. The answer is 'no' on both counts. In particular, in the case $N=1$ and $L$ given by $[7.1$, see [26, 65] and 64, p. 454], there exist almost periodic potentials $b$ for which the spectrum of $A$ as an operator on $Y^{2}(U)$ has the property that every solution $x \in Y^{\infty}(U)$ of $\lambda x=\left(L+M_{c}\right) x$, for some $c \in \operatorname{Lim}(b)$ decays exponentially at infinity.

To illustrate the application of the above theorem in the 1D case $(N=1)$ we consider a widely studied class of almost periodic operators obtained by the following construction. For some $d \in \mathbb{N}$ let $B: \mathbb{R}^{d} \rightarrow L(U)$ be a continuous function satisfying

$$
B(s+m)=B(s), \quad s \in \mathbb{R}^{d}, m \in \mathbb{Z}^{d} .
$$


Let $\alpha=\left(\alpha_{1}, \ldots, \alpha_{d}\right) \in \mathbb{R}^{d}$ and, for $s \in \mathbb{R}^{d}$ let $b_{s}: \mathbb{Z} \rightarrow L(U)$ be given by

$$
b_{s}(n)=B(\alpha n+s), \quad n \in \mathbb{Z} .
$$

If $\alpha_{1}, \ldots, \alpha_{d}$ are all rational, then $b_{s}$ is periodic. Whatever the choice of $\alpha_{1}, \ldots, \alpha_{d}$, $b_{s}$ is almost periodic $\left(b_{s} \in Y_{\mathrm{AP}}^{\infty}(L(U))\right)$.

For $s \in \mathbb{R}^{d}$ let $[s]$ denote the coset $[s]=s+\mathbb{Z}^{d}$ in $\mathbb{R}^{d} / \mathbb{Z}^{d}$. An interesting case is that in which $1, \alpha_{1}, \alpha_{2}, \ldots, \alpha_{d}$ are rationally independent, in which case $\{[\alpha m]: m \in \mathbb{Z}\}$ is dense in $\mathbb{R}^{d} / \mathbb{Z}^{d}$. Then it is a straightforward calculation to see that

$$
\operatorname{Lim}\left(b_{s}\right)=\left\{b_{t}: t \in \mathbb{R}^{d}\right\}
$$

Thus, for this case, (7.7) reads as

$$
\operatorname{spec}_{\mathrm{ess}}^{p}\left(L+M_{b_{s}}\right)=\operatorname{spec}^{p}\left(L+M_{b_{s}}\right)=\bigcup_{t \in \mathbb{R}^{d}} \operatorname{spec}_{\text {point }}^{\infty}\left(L+M_{b_{t}}\right) .
$$

As a particular instance, this formula holds in the case when $U=\mathbb{C}, d=1$, and $B(s)=\lambda \cos (2 \pi s), s \in \mathbb{R}$, for some $\lambda \in \mathbb{C}$. Then

$$
b_{s}(n)=\lambda \cos (2 \pi(\alpha n+s)), \quad n \in \mathbb{Z},
$$

and (7.10) holds if $\alpha$ is irrational, in which case $b_{s}$ is the so-called almost Mathieu potential.

We next modify the above example to illustrate the application of Theorem 7.1 in a particular 1D $(N=1)$ case.

EXAMPLE 7.5 Define $b_{s} \in Y_{\mathrm{AP}}^{\infty}(L(U))$ by $(7.8)$ and suppose that $1, \alpha_{1}, \alpha_{2}, \ldots, \alpha_{d}$ are rationally independent. Suppose that $f: \mathbb{Z} \rightarrow \mathbb{R}^{d}$ satisfies

$$
\lim _{|n| \rightarrow \infty}|f(n+1)-f(n)| \rightarrow 0 .
$$

Define $b \in Y^{\infty}(L(U))$ by

$$
b(n)=B(\alpha n+f(n)), \quad n \in \mathbb{Z} .
$$

Then it is straightforward to see that $\operatorname{Lim}(b) \subset\left\{b_{s}: s \in \mathbb{R}^{d}\right\}$. Since [51, Corollary 3.97], $b_{s} \in \operatorname{Lim}(b)$ implies that $\operatorname{Lim}\left(b_{s}\right) \subset \operatorname{Lim}(b)$, we have, by (7.9), that

$$
\operatorname{Lim}(b)=\left\{b_{s}: s \in \mathbb{R}^{d}\right\} .
$$

Thus, applying Theorem 7.1 and 7.10 , we see that, for every $s \in \mathbb{R}^{d}$ and every $p \in\{0\} \cup[1, \infty]$,

$$
\operatorname{spec}_{\text {ess }}^{p}\left(L+M_{b}\right)=\operatorname{spec}_{\text {ess }}^{p}\left(L+M_{b_{s}}\right)=\operatorname{spec}^{p}\left(L+M_{b_{s}}\right)=\bigcup_{t \in \mathbb{R}^{d}} \operatorname{spec}_{\text {point }}^{\infty}\left(L+M_{b_{t}}\right) .
$$

We note that, in the special case that $L$ is given by (7.1) (with $N=1$ ), $U=\mathbb{C}$, and $B$ is real-valued, the statement that

$$
\operatorname{spec}_{\mathrm{ess}}^{2}\left(L+M_{b}\right)=\operatorname{spec}^{2}\left(L+M_{b_{s}}\right)
$$

for all $s \in \mathbb{R}^{d}$ is Theorem 5.2 of Last and Simon 47. (established by limit operator type arguments). As a specific instance where $\sqrt{7.12}$ holds, let us take $U=\mathbb{C}$, $d=1$, and $B(s)=\lambda \cos (2 \pi s), s \in \mathbb{R}$, for some $\lambda \in \mathbb{C}$. Then $b_{s}$ is given by 7.11) and, taking (as one possible choice), $f(n)=|n|^{1 / 2}$, one has

$$
b(n)=\lambda \cos \left(2 \pi\left(\alpha n+|n|^{1 / 2}\right)\right)
$$


(cf. [47, Theorem 1.3]).

As a further example we consider the case when $b$ is pseudo-ergodic in the sense of Davies [27. Following [27, we call the function $b \in Y^{\infty}\left(\mathbb{Z}^{N}, U\right)$ pseudo-ergodic, if, for every $\varepsilon>0$, every finite set $S \subset \mathbb{Z}^{N}$ and every function $f: S \rightarrow \Sigma:=$ $\operatorname{clos}_{U} b\left(\mathbb{Z}^{N}\right)$, there is a $z \in \mathbb{Z}^{N}$ such that

$$
\|f(s)-b(z+s)\|_{U}<\varepsilon, \quad s \in S .
$$

One can show [51, Corollary 3.70] that $b$ is pseudo-ergodic iff $\operatorname{Lim}(b)$ is the set $\Sigma^{\mathbb{Z}^{N}}$ of all functions $c: \mathbb{Z}^{N} \rightarrow \Sigma$. In particular, $b \in \operatorname{Lim}(b)$ if $b$ is pseudo-ergodic.

THEOREM 7.6. If $b$ is pseudo-ergodic then, for all $p$,

$$
\operatorname{spec}_{\mathrm{ess}}^{p}(A)=\operatorname{spec}^{p}(A)=\bigcup_{c \in \Sigma^{\mathbb{Z}^{N}}} \operatorname{spec}^{p}\left(L+M_{c}\right)=\bigcup_{c \in \Sigma^{\mathbb{Z}^{N}}} \operatorname{spec}_{\text {point }}^{\infty}\left(L+M_{c}\right) .
$$

Proof. The first two '=' signs follow from 7.3 and the fact that $b \in \operatorname{Lim}(b)=\Sigma \Sigma^{\mathbb{Z}^{N}}$. For the proof of the remaining equality, we refer to the following $s$-dense subset of $\operatorname{Lim}(b)=\Sigma^{\mathbb{Z}^{N}}$ : Let $m_{1}=m_{2}=\ldots=m_{N}=1$, and let $\varsigma$ stand for the set of all periodic functions $x: \mathbb{Z}^{N} \rightarrow \Sigma$, that is

$$
\varsigma:=\bigcup_{n \in \mathbb{N}} Y_{n}^{\infty}(\Sigma)
$$

with $Y_{n}^{\infty}(\Sigma)$ defined as in 6.4 (with the slight abuse of notation by writing $Y^{\infty}(\Sigma)$ for $\Sigma^{\mathbb{Z}^{N}}$, i.e. the set of all functions $x: \mathbb{Z}^{N} \rightarrow \Sigma$ ). Then $\varsigma$ is $s$-dense in $\Sigma^{\mathbb{Z}^{N}}$ as every $x \in \Sigma^{\mathbb{Z}^{N}}$ can be strictly approximated by the sequence $\left(\tilde{P}_{n} x\right) \subset \varsigma$ with $\tilde{P}_{n}$ as defined in (6.5). If $\lambda \in \mathbb{C}$ and all limit operators $\lambda I-\left(L+M_{c}\right)$ of $\lambda I-A=\lambda I-\left(L+M_{b}\right)$, including those with $c \in \varsigma$, are injective, then, by Theorem 6.7, we have that $\lambda I-\left(L+M_{c}\right)$ is surjective for every $c \in \varsigma$. By the equivalence between (a) and (d) in Theorem 7.1, this shows that $\lambda I-A=\lambda I-\left(L+M_{b}\right)$ is Fredholm.

REMARK 7.7 It is shown that

$$
\operatorname{spec}_{\text {ess }}^{2}(A)=\operatorname{spec}^{2}(A)=\bigcup_{c \in \Sigma^{\mathbb{Z}^{N}}} \operatorname{spec}^{2}\left(L+M_{c}\right)
$$

in 27. The result that $\operatorname{spec}^{p}(A)=\bigcup_{c \in \Sigma^{\mathbb{Z}^{N}}} \operatorname{spec}_{\text {point }}^{\infty}\left(L+M_{c}\right)$ appears to be new.

The above theorems show that, in each of the cases $L$ symmetric, $b$ almost periodic, and $b$ pseudo-ergodic, it holds that

$$
\operatorname{spec}_{\mathrm{ess}}^{p}(A)=\bigcup_{c \in \operatorname{Lim}(c)} \operatorname{spec}_{\text {point }}^{\infty}\left(L+M_{c}\right) .
$$

We conjecture that, in fact, this equation holds for all $c \in Y^{\infty}(L(U))$. For $N=1$ this is no longer a conjecture, as we showed in Corollary 6.49 (which follows from our more general results in [17, also see Theorem 6.31 above). For $N \geq 2$ however, this is an open problem.

We finish this chapter with an example demonstrating how Theorem 7.6 can be used to compute spectra of Schrödinger operators with random potential $b$.

ExAmple 7.8 Let $N=1, p \in[1, \infty], U=\mathbb{C}$ and take a compact set $\Sigma$ in the complex plane. We compute the spectrum of $A=L+M_{b}$ as an operator on 
$Y=Y^{p}(U)=\ell^{p}(\mathbb{Z})$ where $L=V_{-1}$ is the backward shift and the function values $b(k), k \in \mathbb{Z}$, of the random potential $b$ are chosen independently of each other from the set $\Sigma$. We assume that, for every $\sigma \in \Sigma$ and $\varepsilon>0, \mathbb{P}(|b(k)-\sigma|<\varepsilon)>0$. Then it is easy to see (the argument is sometimes called 'the Infinite Monkey Theorem' and it follows from the Second Borel Cantelli Lemma, see [12, Theorem 8.16] or 25. Theorem 4.2.4]) that, with probability $1, b$ is pseudo-ergodic.

For the calculation of the point spectra in Theorem 7.6 let $c \in \Sigma^{\mathbb{Z}}$ and take $\lambda \in \mathbb{C}$. If $x: \mathbb{Z} \rightarrow \mathbb{C}$ is a nontrivial solution of $\left(L+M_{c}\right) x=\lambda x$ then $x\left(n_{0}\right) \neq 0$ for some $n_{0} \in \mathbb{Z}$ and $x(k+1)=(\lambda-c(k)) x(k)$ for all $k \in \mathbb{Z}$. Note that $\lambda \neq c(k)$ for all $k<n_{0}$ since otherwise $x\left(n_{0}\right)=0$ and, w.l.o.g., suppose that $x\left(n_{0}\right)=1$. As a consequence we get that

$$
x(n)= \begin{cases}\prod_{k=n_{0}}^{n-1}(\lambda-c(k)), & n \geq n_{0}, \\ \prod_{k=n}^{n_{0}-1}(\lambda-c(k))^{-1}, & n<n_{0}\end{cases}
$$

for every $n \in \mathbb{Z}$. Now put, for $r>0$,

$$
\Sigma_{\cup}^{r}:=\bigcup_{\sigma \in \Sigma}(\sigma+r \overline{\mathbb{D}}) \quad \text { and } \quad \Sigma_{\cap}^{r}:=\bigcap_{\sigma \in \Sigma}(\sigma+r \mathbb{D})
$$

with $\mathbb{D}$ denoting the open unit disk in $\mathbb{C}$ and $\overline{\mathbb{D}}$ its closure.

Clearly, if $\lambda \notin \Sigma_{\cup}^{1}$ then $|\lambda-\sigma|>1$ for all $\sigma \in \Sigma$ and hence, for every nontrivial eigenfunction $x$ of $L+M_{c}$, we have that $|x(n)| \rightarrow \infty$ in 7.14 as $n \rightarrow+\infty$, regardless of $c: \mathbb{Z} \rightarrow \Sigma$.

Similarly, if $\lambda \in \Sigma_{\cap}^{1}$ then $|\lambda-\sigma|<1$ for all $\sigma \in \Sigma$ and hence, for every nontrivial eigenfunction $x$ of $L+M_{c},|x(n)| \rightarrow \infty$ in (7.14) as $n \rightarrow-\infty$, regardless of $c: \mathbb{Z} \rightarrow \Sigma$. (Note that $n_{0}$ in (7.14) depends on $c$ and $\lambda$.)

So in both cases, $\left(L+M_{c}\right) x=\lambda x$ has no nontrivial solution $x \in Y^{\infty}$, so $\lambda \notin \operatorname{spec}_{\text {point }}^{\infty}\left(L+M_{c}\right)$ for all $c \in \Sigma^{\mathbb{Z}}$. Now it remains to look at $\lambda \in \Sigma_{\cup}^{1} \backslash \Sigma_{\cap}^{1}$. In this case, let $\sigma, \tau \in \Sigma$ be such that $|\lambda-\sigma| \leq 1 \leq|\lambda-\tau|$, which is possible by the choice of $\lambda$, and put $c(k):=\tau$ for $k<0$ and $c(k):=\sigma$ for $k \geq 0$. Then $c \in \Sigma^{\mathbb{Z}}$ and

$$
x=\left(\cdots,(\lambda-\tau)^{-2},(\lambda-\tau)^{-2}, 1,(\lambda-\sigma)^{1},(\lambda-\sigma)^{2}, \cdots\right) \in Y^{\infty},
$$

with the 1 at position $n_{0}=0$, is an eigenvector of $L+M_{c}$ w.r.t. $\lambda$.

Summarising and using Theorem 7.6 , we get that, with probability 1 ,

$$
\operatorname{spec}^{p} A=\operatorname{spec}_{\mathrm{ess}}^{p} A=\bigcup_{c \in \Sigma^{\mathbb{Z}}} \operatorname{spec}_{\text {point }}^{\infty}\left(L+M_{c}\right)=\Sigma_{\cup}^{1} \backslash \Sigma_{\cap}^{1},
$$

which confirms, in a simpler and more straightforward way, a result of Trefethen, Contedini and Embree [90, Theorem 8.1] (and see [91, Section VIII]). Equation 7.15 is illustrated, for two particular cases, in Figure 7.1. By a simple scaling argument, it moreover follows from $(7.15)$ that, with probability 1 ,

$$
\operatorname{spec}^{p}\left(\varepsilon L+M_{b}\right)=\operatorname{spec}_{\mathrm{ess}}^{p}\left(\varepsilon L+M_{b}\right)=\Sigma_{\cup}^{\varepsilon} \backslash \Sigma_{\cap}^{\varepsilon} .
$$

Thus

$$
\operatorname{spec}^{p}\left(\varepsilon L+M_{b}\right)=\Sigma_{\cup}^{\varepsilon}=\operatorname{spec}_{\varepsilon}\left(M_{b}\right)
$$

for all $\varepsilon>0$ small enough that $\Sigma_{\cap}^{\varepsilon}=\varnothing$, e.g. for $\varepsilon \in(0$, diam $\Sigma / 2)$. 


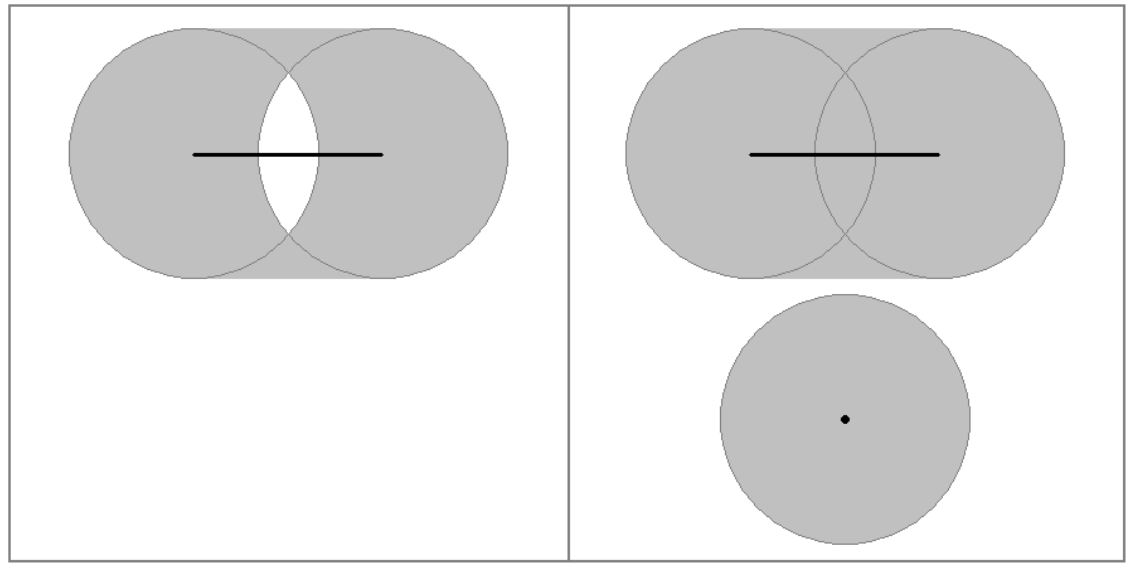

FigurE 7.1. The left image shows, as a gray shaded area, $\operatorname{spec}^{p} A$ when $\Sigma$ is the black straight line of length 1.5. In the right image, one more point (the centre of the lower circle) has been added to $\Sigma$ which results in $\Sigma_{\cap}^{1}=\varnothing$. 


\section{CHAPTER 8}

\section{A Class of Integral Operators}

In this chapter, we apply the results of Chapter 6 to study a class of operators on

$$
Y=Y^{p}(U)= \begin{cases}\ell^{p}\left(\mathbb{Z}^{N}, U\right), & p \in[1, \infty] \\ c_{0}\left(\mathbb{Z}^{N}, U\right), & p=0\end{cases}
$$

with $U=L^{q}\left([0,1]^{N}\right)$ for some fixed $q \in(1, \infty]$. In a natural way (cf. [43, §1.6.3]) we identify elements $x \in Y$ with equivalence classes of scalar-valued functions on $\mathbb{R}^{N}$ via

$$
(x(m))(t)=f(m+t), \quad m \in \mathbb{Z}^{N}, t \in[0,1]^{N},
$$

and denote the set of all of these (equivalence classes of) functions $f$ with

$$
\|f\|_{p, q}:=\|x\|_{Y}<\infty
$$

by $L^{p, q}\left(\mathbb{R}^{N}\right)$ or just $L^{p, q}$. Note that $L^{q, q}\left(\mathbb{R}^{N}\right)=L^{q}\left(\mathbb{R}^{N}\right)$. Equipped with the norm $\|\cdot\|_{p, q}, L^{p, q}$ is a Banach space, and (8.1) yields an isometric isomorphism between $L^{p, q}$ and $Y^{p}(U)$. We will freely identify these two spaces and the notions of strict convergence, limit operators, as well as the operators $P_{m}, V_{k} \in L\left(Y^{p}(U)\right)$ with the corresponding notions and operators on $L^{p, q}$ (cf. [51, (1.3)]).

The operators we are going to study on $Y$ alias $L^{p, q}$ are composed, via addition and composition, from two basic ingredients:

- For $b \in L^{\infty}:=L^{\infty}\left(\mathbb{R}^{N}\right)$, define the multiplication operator $\mathrm{M}_{b} \in L\left(L^{p, q}\right)$ by

$$
\mathrm{M}_{b} f(t)=b(t) f(t), \quad t \in \mathbb{R}^{N}
$$

for all $f \in L^{p, q}$. Via the identification (8.1) between $L^{p, q}$ and $Y$, we can identify $\mathrm{M}_{b}$ with the multiplication operator $M_{c}$ on $Y$ as defined in (4.4), where $c \in \ell^{\infty}\left(\mathbb{Z}^{N}, L(U)\right)$ is such that $(c(m) u)(t)=b(m+t) u(t)$ for all $m \in \mathbb{Z}^{N}, u \in U=L^{q}\left([0,1]^{N}\right)$ and $t \in[0,1]^{N}$. Recall from Example 6.6 that $\mathrm{M}_{b}$ is rich iff $\{b(\cdot+k)\}_{k \in \mathbb{Z}^{N}}$ is relatively sequentially compact in the strict topology on $L^{\infty}$, in which case we write $b \in L_{\$}^{\infty}$. It is easy to check that $L_{\$}^{\infty}$ is an inverse closed Banach subalgebra of $L^{\infty}$.

- For $\kappa \in L^{1}:=L^{1}\left(\mathbb{R}^{N}\right)$, define the convolution operator $C_{\kappa} \in L\left(L^{p, q}\right)$ by

$$
\mathrm{C}_{\kappa} f(t)=(\kappa \star f)(t)=\int_{\mathbb{R}^{N}} \kappa(s-t) f(s) d s, \quad t \in \mathbb{R}^{N}
$$

for all $f \in L^{p, q}$ (cf. Example 3.6). As demonstrated in [51, Example 1.28], the convolution operator $\mathrm{C}_{\kappa}$ on $L^{p, q}$ corresponds to a Laurent operator 
$L$ on $Y$, where every entry $\lambda_{k}$ of $[L]=\left[\lambda_{i-j}\right]_{i, j \in \mathbb{Z}^{N}}$ is the operator of convolution by $\kappa(\cdot+k)$ on $U$. By Young's inequality, we get that

$$
\left\|\lambda_{k}\right\| \leq\left\|\left.\kappa\right|_{k+[-1,1]^{N}}\right\|_{1}
$$

for every $k \in \mathbb{Z}^{N}$, and by $\kappa \in L^{1}\left(\mathbb{R}^{N}\right)$ it follows that $L \in \mathcal{W}=\mathcal{W}(U)$.

We denote by $\mathcal{A}^{o}$ the smallest algebra in $L\left(L^{p, q}\right)$ containing all operators of these two types; that is the set of all finite sum-products of operators of the form $\mathrm{M}_{b}$ and $\mathrm{C}_{\kappa}$ with $b \in L_{\$}^{\infty}$ and $\kappa \in L^{1}$. From the above considerations it follows that every operator $A \in \mathcal{A}^{\circ}$, if identified with an operator on $Y$, is contained in the Wiener algebra $\mathcal{W}$. By $\mathcal{A}$ we denote the closure of $\mathcal{A}^{o}$ in the norm $\|.\|_{\mathcal{W}}$. Note that, by 6.33), the closure of a set $S \subset \mathcal{W}$ in the $\mathcal{W}$-norm is always contained in the closure of $S$ in the usual operator norm.

Lemma 8.1. The predual space $U^{\triangleleft}$ exists and, if $p=\infty$, then every $A \in \mathcal{A}$ has a preadjoint operator $A^{\triangleleft}$ on $L^{1, q^{\prime}}$ with $1 / q+1 / q^{\prime}=1$.

Proof. By the choice $q \in(1, \infty]$, it is clear that the predual space $U^{\triangleleft}$ of $U=$ $L^{q}\left([0,1]^{N}\right)$ exists and can be identified with $L^{q^{\prime}}\left([0,1]^{N}\right)$, where $1 / q+1 / q^{\prime}=1$, including the case $q^{\prime}=1$ if $q=\infty$. Now suppose $p=\infty$. Then the predual $Y^{1}\left(U^{\triangleleft}\right)$ of $Y=Y^{\infty}(U)$ exists and corresponds to $L^{1, q^{\prime}}$ in the sense of 8.1]. By [51, Proposition 1.10] and the fact that both multiplication and convolution operators have a preadjoint operator (indeed, $\mathrm{M}_{b}^{\triangleleft}=\mathrm{M}_{b}$ and $\mathrm{C}_{\kappa}^{\triangleleft}=\mathrm{C}_{\kappa(-)}$ ) for all $b \in L^{\infty}$ and $\kappa \in L^{1}$ ), we see that indeed $A^{\triangleleft}$ exists for every $A \in \mathcal{A}$.

Now let

$$
\mathcal{J}^{o}:=\left\{\sum A_{i} C_{\kappa_{i}} B_{i}: A_{i}, B_{i} \in \mathcal{A}^{o}, \kappa_{i} \in L^{1}\right\},
$$

with the sum being finite, denote the smallest two-sided ideal of $\mathcal{A}^{o}$ containing all convolution operators $C_{\kappa}$ with $\kappa \in L^{1}$, and let $\mathcal{J}$ be its closure in the norm $\|.\|_{\mathcal{W}}$, hence the smallest $\mathcal{W}$-closed two-sided ideal of $\mathcal{A}$ containing all $\mathrm{C}_{\kappa}$.

Lemma 8.2. It holds that $\mathcal{J} \subset U M\left(L^{p, q}\right)$. In particular, every operator in $\mathcal{J}$ is Montel.

Proof. The inclusion $\mathcal{J} \subset U M\left(L^{p, q}\right)$ follows from Lemma 6.24 (a),(c),(d) and the fact that $\mathrm{C}_{\kappa} \in U M\left(L^{p, q}\right)$ for all $\kappa \in L^{1}$ since the set $\left\{V_{-k} K V_{k}: k \in \mathbb{Z}^{N}\right\}$ in Lemma 6.23 (ii) is just a singleton if $K=\mathrm{C}_{\kappa}$.

It can be shown that, in the same way as every $A \in \mathcal{A}^{o}$ clearly can be written as the sum of a multiplication operator and an operator in $\mathcal{J}^{\circ}$, also every $A \in \mathcal{A}$ can be uniquely written as

$$
A=\mathrm{M}_{b}+K \quad \text { with } \quad b \in L_{\$}^{\infty} \quad \text { and } \quad K \in \mathcal{J} .
$$

This follows from [51, Proposition 4.11] with $\mathcal{A}$ and $\mathcal{J}$ there replaced by the current meaning. As a consequence, we get that the factor algebra $\mathcal{A} / \mathcal{J}$ is isomorphic to $L_{\$}^{\infty}$, and the coset $A+\mathcal{J}$ of $A \in \mathcal{A}$ is represented by the function $b \in L_{\$}^{\infty}$ from 8.2 .

THEOREM 8.3. The operator 8.2) is Fredholm iff it is invertible at infinity and $b$ is invertible in $L^{\infty}$. 
Proof. If $A \in \mathcal{A}$ is invertible at infinity and $b$ from its representation 8.2 is invertible in $L^{\infty}$, then $A$ is Fredholm by Theorem 6.28 (ii) and Lemma 8.2 . Conversely, let $A \in \mathcal{A}$ be Fredholm. By Theorem 6.28 (i) and b), together with Lemma 8.1 we get that $A$ is invertible at infinity. It remains to show that $b$ from 8.2 is invertible in $L^{\infty}$. To see this, take $B \in L\left(L^{p, q}\right)$ and $S, T \in K\left(L^{p, q}\right)$ such that $A B=I+S$ and $B A=I+T$. Then, for every $k \in \mathbb{N}$, we get that

$$
P_{k} \mathrm{M}_{b} B P_{k}+P_{k} K B P_{k}=P_{k} A B P_{k}=P_{k}+P_{k} S P_{k},
$$

and hence

$$
\left(P_{k} \mathrm{M}_{b} P_{k}\right)\left(P_{k} B P_{k}\right)=P_{k}+S^{\prime}
$$

with $S^{\prime}=P_{k} S P_{k}-P_{k} K B P_{k} \in K\left(L^{p, q}\left([-k, k]^{N}\right)\right)$ by Lemmas 8.2 and 3.19 . From the last equality and its symmetric counter-part, we conclude that $\mathrm{M}_{P_{k} b}=P_{k} \mathrm{M}_{b} P_{k}$ is Fredholm on $L^{p, q}\left([-k, k]^{N}\right)$, implying that the function $P_{k} b$ is invertible in $L^{\infty}\left([-k, k]^{N}\right)$, by a standard argument (see e.g. [51, Lemma 2.42]). Since this holds for every $k \in \mathbb{N}$, we get that $b$ is invertible in $L^{\infty}$.

As invertibility of $b$ in $L^{\infty}$ turned out to be necessary for Fredholmness of (8.2), we will now, without loss of generality, suppose that $b$ is invertible, therefore write

$$
A=\mathrm{M}_{b}+K=\mathrm{M}_{b}\left(I+K^{\prime}\right) \quad \text { with } \quad K^{\prime}=\mathrm{M}_{b^{-1}} K \in \mathcal{J},
$$

and then merely study Fredholmness of $I+K^{\prime}$. For this setting we can show the analogous result of Theorem 7.1 .

TheOREM 8.4. For $A=I+K$ with $K \in \mathcal{J}$, the following statements are equivalent.

(a) All limit operators of $A$ are injective on $L^{\infty, q}$ and $\sigma^{\mathrm{op}}(A)$ has an $S$-dense subset of injective operators on $L^{1, q}$;

(b) All limit operators of $A$ are invertible on one of the spaces $L^{p, q}$ with $p \in$ $\{0\} \cup[1, \infty]$;

(c) All limit operators $A_{h}$ of $A$ are invertible on all the spaces $L^{p, q}$ with $p \in$ $\{0\} \cup[1, \infty]$ and the inverses are uniformly bounded (in $p$ and $h$ );

(d) $A$ is Fredholm on one of the spaces $L^{p, q}$ with $p \in\{0\} \cup[1, \infty]$;

(e) $A$ is Fredholm on all the spaces $L^{p, q}$ with $p \in\{0\} \cup[1, \infty]$.

Thus, for every $p \in\{0\} \cup[1, \infty]$ it holds that

$$
\begin{aligned}
\operatorname{spec}_{\mathrm{ess}}^{p}(A) & =\bigcup_{A_{h} \in \sigma^{\text {op }}(A)} \operatorname{spec}^{p}\left(A_{h}\right) \\
& =\bigcup_{A_{h} \in \sigma^{\text {op }}(A)}\left[\operatorname{spec}_{\text {point }}^{\infty}\left(A_{h}\right) \cup \operatorname{spec}_{\text {point }}^{1}\left(A_{h}\right)\right]
\end{aligned}
$$

and

$$
\begin{aligned}
\operatorname{spec}^{p}(A) & =\operatorname{spec}_{\text {point }}^{\infty}(A) \cup \operatorname{spec}_{\text {point }}^{1}\left(A^{\prime}\right) \cup \operatorname{spec}_{\text {ess }}^{p}(A) \\
& =\operatorname{spec}_{\text {point }}^{1}(A) \cup \operatorname{spec}_{\text {point }}^{1}\left(A^{\prime}\right) \cup \operatorname{spec}_{\text {ess }}^{p}(A) .
\end{aligned}
$$

Proof. We start by showing that $A$ is subject to the conditions in Corollary 6.45 . Clearly, $A=I+K$ is contained in the Wiener algebra and it is rich, by $(5.13)$ and since all generators $\mathrm{M}_{b}$ and $\mathrm{C}_{\kappa}$ of $\mathcal{A}$ are rich. Predual $U^{\triangleleft}$ and preadjoint $A^{\triangleleft}$ exist by Lemma 8.1, and $K \in U M\left(L^{p, q}\right)$ by Lemma 8.2 . 
The rest of this proof proceeds exactly as that of Theorem 7.1 with one difference: Unlike Theorem 7.1, which rests on Corollary 6.46, we here have an infinitedimensional space $U$ and therefore we use Corollary 6.45

EXAMPLE 8.5 The spectra of limit operators of $A=I+K$ can be written down explicitly when $K \in \mathcal{J}$ is composed of convolution operators $C_{\kappa}$ with $\kappa \in L^{1}$ and multiplication operators $\mathrm{M}_{b}$ with a slowly oscillating function $b \in L^{\infty}$. By the latter we mean that

$$
\operatorname{ess~sup}_{t \in[0,1]^{N}}|b(x+t)-b(x)| \rightarrow 0 \quad \text { as } \quad|x| \rightarrow \infty .
$$

In this case, the multiplication operator $M_{b}$ is rich and all of its limit operators are multiples of the identity. (In a sense, even the reverse statement is true [51, Proposition 3.52].) As a consequence, every limit operator $A_{h}$ of $A=I+K$ is of the form $I+C_{\kappa}$ with some $\kappa \in L^{1}$, in which case the set $\operatorname{spec}^{p}\left(A_{h}\right)$ is the range of the function $1+F \kappa$ with $F$ being the Fourier transform on $L^{1}$.

One can now proceed similarly to Chapter 7 to get rid of the second injectivity condition in Theorem 8.4 (a) and the second point-spectrum in formula 8.3 ): Clearly, if $N=1$, then Corollary 6.48 does exactly this for us. Otherwise, if $N \geq 2$, one way to go is to restrict ourselves to symmetric operators in $\mathcal{A}$. We will illustrate another way, that is restricting the generating multiplication operators $\mathrm{M}_{b}$ of $\mathcal{A}$ to norm-rich/almost periodic ones, i.e. to work with a type of almost periodic functions $b \in L^{\infty}$.

Let $b \in L^{\infty}$ and put $c=\left(\left.b\right|_{k+[0,1]^{N}}\right)_{k \in \mathbb{Z}^{N}} \in Y^{\infty}\left(L^{\infty}\left([0,1]^{N}\right)\right)$. From Example 6.6 we know that the following are equivalent:

- The set $\left\{V_{k} b: k \in \mathbb{Z}^{N}\right\}$ is relatively compact in $L^{\infty}$.

- The set $\left\{V_{k} c: k \in \mathbb{Z}^{N}\right\}$ is relatively compact in $Y^{\infty}\left(L^{\infty}\left([0,1]^{N}\right)\right)$.

- $c$ is almost periodic, i.e. $c \in Y_{\mathrm{AP}}^{\infty}\left(L^{\infty}\left([0,1]^{N}\right)\right)$.

- $M_{c}$ is norm-rich/almost periodic (and therefore rich) on $Y^{p}(U)$.

When this is the case, then we will say that $b \in L^{\infty}$ is $\mathbb{Z}$-almost periodic and write $b \in L_{\mathrm{AP}}^{\infty}$. The set of $\mathbb{Z}$-almost periodic functions on $\mathbb{R}^{N}$ is not to be confused with the much smaller subset of almost periodic functions on $\mathbb{R}^{N}$. Unlike almost periodic functions, the functions in $L_{\mathrm{AP}}^{\infty}$ do not need to be continuous (see [51, §3.4.8] for more detail).

So let $\mathcal{A}_{\mathrm{AP}} \subset \mathcal{A}$ denote the $\mathcal{W}$-closure of the smallest algebra in $L\left(L^{p, q}\right)$ that contains all $\mathrm{M}_{b}$ with $b \in L_{\mathrm{AP}}^{\infty}$ and all $\mathrm{C}_{\kappa}$ with $\kappa \in L^{1}$. Analogously, $\mathcal{J}_{\mathrm{AP}}$ be the smallest $\mathcal{W}$-closed two-sided ideal in $\mathcal{A}_{\mathrm{AP}}$ containing all convolution operators $\mathrm{C}_{\kappa}$ with $\kappa \in L^{1}$. It is not hard to see that $\mathcal{J}_{\mathrm{AP}}=\mathcal{J} \cap \mathcal{A}_{\mathrm{AP}}$.

As seen before for operators in $\mathcal{A}$, the study of Fredholmness in $\mathcal{A}_{\mathrm{AP}}$ can be reduced to studying operators of the form $I+K$ with $K \in \mathcal{J}_{\text {AP. }}$ In this case, in full analogy to Theorem 7.3 , we have the following improved version of formula (8.3):

TheOREM 8.6. If $A=I+K$ with $K \in \mathcal{J}_{\mathrm{AP}}$ then, for all $p \in\{0\} \cup[1, \infty]$ and all $A_{h} \in \sigma^{\mathrm{op}}(A)$,

$$
\operatorname{spec}_{\mathrm{ess}}^{p}(A)=\operatorname{spec}^{p}(A)=\operatorname{spec}^{p}\left(A_{h}\right)=\bigcup_{B \in \sigma^{\mathrm{op}}(A)} \operatorname{spec}_{\text {point }}^{\infty}(B) .
$$


Proof. First of all, $A$ is norm-rich/almost periodic, by Lemma 6.9 and since the generators of $\mathcal{A}_{\mathrm{AP}}$ are norm-rich/almost periodic. Secondly, $\sigma^{\mathrm{oP}}(K)$ is uniformly Montel, by Lemmas 8.2 and 5.17. Consequently, we may use Theorems 6.38 and 6.10 (iv), which, together with the equivalence of statements (b) and (d) in Theorem 8.4. prove this formula. 


\section{CHAPTER 9}

\section{Some Open Problems}

We conclude with a small list of open problems the solutions of which, we believe, could be crucial in extending the picture that we have tried to draw in this text. We would like to see this list as both a future agenda for ourselves as well as an invitation for the interested reader.

1. Is a version of the limit operator theory possible with $L(Y, \mathcal{P})$ and $K(Y, \mathcal{P})$ replaced by $S(Y)$ and $S N(Y)$ ? We know from Lemma 3.3. Corollary 3.5. (3.5) and Lemma 3.10 that $S(Y)$ and $S N(Y)$ are "one-sided versions" of $L(Y, \mathcal{P})$ and $K(Y, \mathcal{P})$. Moreover, $S N(Y)$ is a closed two-sided ideal in $S(Y)$ - just like $K(Y, \mathcal{P})$ is in $L(Y, \mathcal{P})$. The ideal $K(Y, \mathcal{P})$ shapes the theory presented here in two ways: It defines the notion of invertibility at infinity (see Definition 5.1) and that of $\mathcal{P}$ convergence (see Definition 4.1). How do these properties change if one works with the ideal $S N(Y)$ instead and what is the connection between the new notions of 'invertibility at infinity' and 'limit operator'?

2. A related but rather different question is the following: To what extent is a version of the limit operator theory possible with what we termed 'weak limit operators' in our discussion of the papers $[\mathbf{5 9}, 6 \mathbf{6 0}$, on page 8 ? To be precise, to what extent is a version of the limit operator theory possible replacing $\mathcal{P}$-convergence $(\stackrel{\mathcal{P}}{\rightarrow})$ in Definition 5.10 of a limit operator by the weaker $\stackrel{s}{\rightarrow}$ convergence of operators introduced in (4.6) in Chapter 4? This question is of interest because weakening the concept of a limit operator widens the class of operators that are rich, and so potentially widens the applicability of the results in this text. Indications that versions of at least some of our results of Chapter 6 may hold in this context are: the results of Muhamadiev [59, 6 , that we discuss on page 8 , and that it is the weaker $\stackrel{s}{\rightarrow}$ convergence rather than $\stackrel{\mathcal{P}}{\rightarrow}$ which is required in the theorems in Section 5.4 .

3. Is $S(Y)$ inverse closed in $L(Y)$ ? We just mentioned that $S(Y)$ is a "onesided version" of $L(Y, \mathcal{P})$ and we know from [74, Theorem 1.1.9] that $L(Y, \mathcal{P})$ is inverse closed. Looking back to problem 1, it seems a sensible question to ask whether also $S(Y)$ is inverse closed. In Section 3.2 we were able to prove that if $A$ is invertible in $S(Y)$ and $B=A+K \in S(Y)$ is a Montel perturbation that is invertible in $L(Y)$ then $B^{-1} \in S(Y)$. This fact implies inverse closedness of $S(Y)$ in cases where $Y=\hat{Y}$ and $P_{n} \in K(Y)$ for all $n$.

4. Is the condition of existence of a predual $U^{\triangleleft}$ and preadjoint $A^{\triangleleft}$ redundant in Theorem 6.17? ...and hence in Theorems 6.28 and 6.44 and Corollaries 6.32 . 
6.43 6.45 and 6.48. Recall that we use the existence of predual and preadjoint to conclude Fredholmness of $A_{0}=\left.A\right|_{Y_{0}}$ from that of $A$ on $Y^{\infty}$, including preservation of the index.

5. Is the hyperplane condition redundant in Theorem 6.44? This possibility has been discussed in the final section of $\mathbf{5 2}$. The answer is positive if the following conjecture holds: $\varphi\left(Y^{p}(U)\right)=\varphi(U)$, where $\varphi(X)$ denotes the smallest positive integer $\kappa$ for which a Fredholm operator of index $\kappa$ exists on the Banach space $X$ (with $\varphi(X)=0$ if all Fredholm operators on $X$ have index zero).

6. Is there a version of Theorem 6.31 in arbitrary dimensions $N \geq 1$ ? As we sketched briefly in the intro to Theorem 6.31. the proof [17. of this result consists of three steps. In two of these steps we use the fact that $N=1$ and it is not clear to us whether and how this condition could be removed. However, we are optimistic that the result also holds for $N \geq 1$.

7. What does Theorem 5.20 have to say about the stability of classical approximation methods for operator equations (e.g. finite section and discretisation methods)? We have not tackled this topic in this text, but some results in this direction are in $13,20,56,18$ (and see $[5,80,66,36,72,73,37,48,74,54,51,16,79$ ).

And finally the classic:

8. Is the uniform boundedness condition in Theorem 6.28 (iii) also redundant for $p \in(1, \infty)$ ? This question is as old as the first versions of Theorem 6.28 (iii). For $p \in\{0,1, \infty\}$ the redundancy was shown in [49, 51] and in this text. For the remaining cases $p \in(1, \infty)$ the question remains open. See [49, Section 3.3] and [51, Section 3.9] for a little survey on this question and some humble attempts to tackle it. 


\section{Bibliography}

[1] R. P. Agarwal and D. O'Regan: Infinite Interval Problems for Differential, Difference and Integral Equations, Kluwer Academic Publishers, Dordrecht, Boston, London 2001.

[2] G. R. Allan: Ideals of vector-valued functions, Proc. London Math. Soc., 18 (1968), 193216.

[3] P. M. Anselone: Collectively Compact Operator Approximation Theory and Applications to Integral Equations, Prentice-Hall, Englewood Cliffs 1971.

[4] P. M. Anselone and J. W. LEE: Nonlinear integral equations on the half-line, J. Integral Equations Appl., 4 (1992), 1-14.

[5] P. M. Anselone and I.H. SloAn: Integral equations on the half-line, J. Integral Eq. 9 (1985), $3-23$.

[6] T. Arens, S. N. Chandler-Wilde and K. Haseloh: Solvability and spectral properties of integral equations on the real line: I. weighted spaces of continuous functions, Journal of Mathematical Analysis and Appl. 272 (2002), 276-302.

[7] T. Arens, S. N. Chandler-Wilde and K. Haseloh: Solvability and spectral properties of integral equations on the real line. II. $L^{p}$-spaces and applications, J. Integral Equations Appl. 15 (2003), no. 1, 1-35.

[8] J. Asch and A. Joye (Eds.): Mathematical Physics of Quantum Mechanics, Springer-Verlag, Heidelberg, 2006.

[9] A. Böttcher, Yu. I. Karlovich and V. S. Rabinovich: The method of limit operators for one-dimensional singular integrals with slowly oscillating data, J. Operator Theory, 43 (2000), 171-198.

[10] R. C. Buck: Bounded Continuous Functions on a Locally Compact Space, Michigan Math. J. 5 (1958), 95-104.

[11] R. CARmona and J. Lacroix: Spectral Theory of Random Schrödinger Operators, Birkhäuser, Boston, 1990.

[12] M. Capinski and E. P. Kopp: Measure, Integral and Probability, Springer Verlag 2004.

[13] S. N. Chandler-Wilde: Some uniform stability and convergence results for integral equations on the real line and projection methods for their solution. IMA Journal of Numerical Analysis 13 (1993), 509-535.

[14] S. N. Chandler-Wilde: The impedance boundary value problem for the Helmholtz equation in a half-plane, Mathematical Methods in the Applied Sciences 20 (1997), 813-840.

[15] S. N. Chandler-Wilde and M. Lindner: Wave problems in unbounded domains: Fredholmness and the finite section method, Contemporary Mathematics (AMS) 440 (2007), 115-122.

[16] S. N. Chandler-Wilde and M. Lindner: Boundary integral equations on unbounded rough surfaces: Fredholmness and the Finite Section Method, J. Integral Eq. and Appl. 20 (2008), $13-48$.

[17] S. N. Chandler-Wilde and M. Lindner: Sufficiency of Favard's condition for a class of band-dominated operators on the axis, J. Funct. Anal. 254 (2008), 1146-1159.

[18] S. N. Chandler-Wilde, M. Rahman and C. R. Ross: A fast two-grid and finite section method for a class of integral equations on the real line with application to an acoustic scattering problem in the half-plane, Numerische Mathematik, 93 (2002), no. 1, 1-51.

[19] S. N. Chandler-Wilde, C. R. Ross and B. Zhang: Scattering by infinite one-dimensional rough surfaces. Proceedings of the Royal Society of London, Series A 455 (1999) 3767-3787.

[20] S. N. Chandler-Wilde and B. Zhang: On the solvability of a class of second kind integral equations on unbounded domains. Journal of Mathematical Analysis and Applications 214 (1997), 482-502. 
[21] S. N. Chandler-Wilde and B. Zhang: Electromagnetic scattering by an inhomogeneous conducting or dielectric layer on a perfectly conducting plate. Proceedings of the Royal Society of London, Series A 454 (1998) 519-542.

[22] S N ChandleR-Wilde and B Zhang: Scattering of electromagnetic waves by rough interfaces and inhomogeneous layers. SIAM Journal on Mathematical Analysis, 30 (1999) 559-583.

[23] S. N. Chandler-Wilde and B. Zhang: A generalised collectively compact operator theory with an application to second kind integral equations on unbounded domains, J. Integral Equations Appl. 14 (2002), 11-52.

[24] S. N. Chandler-Wilde, B. Zhang and C. R. Ross: On the solvability of second kind integral equations on the real line. Journal of Mathematical Analysis and Applications 245, (2000), 28-51.

[25] K. L. Chung: A Course in Probability Theory, 3rd ed., Academic Press 2001.

[26] W. Craig: Pure point spectrum for discrete Schrödinger operators, Commun. Math. Phys. 88 (1983), 113-131.

[27] E. B. DAvies: Spectral theory of pseudo-ergodic operators, Commun. Math. Phys. 216 (2001), 687-704.

[28] E. B. Davies: Linear Operators and their Spectra, Cambridge University Press, 2007.

[29] J. D. DePree and J. A. Higgins: Collectively compact sets of linear operators, Mathemat. Zeitschrift, Mathematics and Statistics 115, no. 5 (1970), 366-370.

[30] J. D. DePree and H. S. KLein: Characterization of collectively compact sets of linear operators, Pacific J. Math. 55, no. 1 (1974), 45-54.

[31] J. D. DePree and H. S. Klein: Semi-groups and collectively compact sets of linear operators, Pacific J. Math. 55, no. 1 (1974), 55-63.

[32] R. G. Douglas: Banach Algebra Techniques in Operator Theory, Academic Press, New York, London 1972.

[33] J. A. FAVARD: Sur les equations differentiales a coefficients presque periodiques, Acta Math. 51 (1927), 31-81.

[34] I. Gohberg, S. Goldberg and M. A. Kanshoek: Classes of Linear Operators, Vol. 1 of 2, Birkhäuser 1993.

[35] W.T. Gowers: A solution of Banach's hyperplane problem, Bull. LMS 26 (1994), 523-530.

[36] R. Hagen, S. Roch and B. Silbermann: Spectral Theory of Approximation Methods for Convolution Equations, Birkhäuser Verlag, 1995.

[37] R. Hagen, S. Roch and B. Silbermann: $C^{*}-$ Algebras and Numerical Analysis, Marcel Dekker, Inc., New York, Basel, 2001.

[38] K. Jörgens: Linear Integral Operators, Pitman, Boston, 1982.

[39] A. V. KozAK and I. V. Simonenko: Projectional methods for solving multidimensional discrete equations in convolutions, Sib. Mat. Zh. 21 (1980), 119-127.

[40] P. Kuchment: On the Floquet theory of periodic difference operators, in Geometrical and Algebraical Aspects in Several Complex Variables (Cetraro, 1989), 201-209, Sem. Conf. 8, EditEl, Rende, 1991.

[41] P. Kuchment: Floquet theory for partial differential equations, Operator Theory Advances and Applications, 60, Birkhäuser, 1993.

[42] V. G. Kurbatov: Om the invertibility of almost periodic operators, Math. USSR Sbornik, 67 (1990), 367-377,

[43] V. G. Kurbatov: Functional Differential Operators and Equations, Kluwer Academic Publishers, Dordrecht, Boston, London 1999.

[44] B. V. LANGE and V. S. Rabinovich: On the Noether property of multidimensional discrete convolutions, Mat. Zametki 37 (1985), no. 3, 407-421 (Russian, English transl. Math. Notes 37 (1985), 228-237).

[45] B. V. LANGE and V. S. Rabinovich: On Noetherian multidimensional convolution operators with bounded measurable coefficients, Izv. VUZ Matemat. 29 (1985), no. 6, 22-30 (Russian).

[46] B. V. LAnge and V. S. Rabinovich: Pseudo-differential operators on $\mathbb{R}^{n}$ and limit operators, Mat. Sbornik 129 (1986), no. 2, 175-185 (Russian, English transl. Math. USSR Sbornik 577 (1987), no. 1, 183-194).

[47] Y. LAST and B. Simon: The essential spectrum of Schrödinger, Jacobi and CMV operators, J. Anal. Math. 98 (2006) 183-220. 
[48] M. Lindner: The Finite Section Method in the Space of Essentially Bounded Functions: An Approach Using Limit Operators, Numerical Functional Analysis and Optimization 24 (2003), no. $7 \& 8,863-893$.

[49] M. Lindner: Limit Operators and Applications on the Space of Essentially Bounded Functions, PhD Dissertation, TU Chemnitz, 2003.

[50] M. Lindner: Classes of Multiplication Operators and Their Limit Operators, Analysis and its Applications 23 (2004), no. 1, 187 - 204.

[51] M. Lindner: Infinite Matrices and their Finite Sections: An Introduction to the Limit Operator Method, Frontiers in Mathematics, Birkhäuser 2006.

[52] M. Lindner: Fredholmness and index of operators in the Wiener algebra are independent of the underlying space, accepted at Operators and Matrices 2008.

[53] M. Lindner: Fredholmness and index of operators in the Wiener algebra are independent of the underlying space (extended version of [52]), Preprint 2008-01, TU Chemnitz.

[54] M. Lindner, V. S. Rabinovich and S. Roch: Finite sections of band operators with slowly oscilating coefficients, Linear Algebra and Applications 390 (2004), 19-26.

[55] M. Lindner and B. Silbermann: Invertibility at infinity of band-dominated operators in the space of essentially bounded functions, in: Operator Theory: Advances and Applications The Erhard Meister Memorial Volume (2003)

[56] A. Meier and S. N. Chandler-Wilde: On the stability and convergence of the finite section method for integral equation formulations of rough surface scattering, Math. Methods Appl. Sci., 24 (2001), no. 4, 209-232.

[57] E.M. Muhamadiev: On invertibility of differential operators in the space of continuous functions bounded on the real axis, Soviet Math Dokl. 12 (1971), 49-52.

[58] E.M. Muhamadiev: On the invertibility of elliptic partial differential operators, Soviet Math Dokl. 13 (1972), 1122-1126.

[59] E. M. Muhamadiev: On normal solvability and Noether property of elliptic operators in spaces of functions on $\mathbb{R}^{n}$, Part I: Zapiski nauchnih sem. LOMI 110 (1981), 120-140 (Russian, English transl. J. Soviet Math. 25 (1984), No. 1, 884-901).

[60] E. M. Muhamadiev: On normal solvability and Noether property of elliptic operators in spacesof functions on $\mathbb{R}^{n}$, Part II: Zapiski nauchnih sem. LOMI 138 (1984), 108-126 (Russian, English transl. J. Soviet Math. 32 (1986), No. 5, 499-512).

[61] D. Natroshvili, T. Arens and S. N. Chandler-Wilde: Uniqueness, existence, and integral equation formulations for interface scattering problems. Memoirs on Differential Equations and Mathematical Physics 30 (2003) 105-146.

[62] D. O'Regan: Approximation of solutions of nonlinear operator equation on the half-line, Computers Math. Appl., 35 (1998), 65-77.

[63] A. T. Peplow and S. N. Chandler-Wilde: Approximate solution of second kind integral equations on infinite cylindrical surfaces. SIAM Journal on Numerical Analysis 32 (1995), 594-609.

[64] L. Pastur and A. Figotin: Spectra of Random and Almost-Periodic Operators, Springer, Berlin 1992

[65] J. Pöschl: Examples of discrete Schrödinger operators with pure point spectrum, Commun. Math. Phys. 88 (1983), 447-463.

[66] S. Prössdorf and B. Silbermann: Numerical Analysis for Integral and Related Operator Equations, Akademie-Verlag, Berlin, 1991 and Birkhäuser Verlag, Basel, Boston, Berlin 1991.

[67] V. S. Rabinovich: Fredholmness of pseudo-differential operators on $\mathbb{R}^{n}$ in the scale of $L_{p, q^{-}}$ spaces. Siberian Math. J. 29 (1988), no. 4, 635-646 (Russian).

[68] V. S. Rabinovich: Operator-valued discrete convolutions and some of its applications, Mat. Zametki 51 (1992), no. 5, 90-101 (Russian).

[69] V. S. Rabinovich and S. Roch: The essential spectrum of Schrödinger operators on lattices, J. Pnys. A: Math. Gen. 39 (2006) 8377-9394.

[70] V. S. Rabinovich and S. Roch: The Fredholm index of locally compact band-dominated operators on $L^{p}(\mathbb{R})$, Preprint Nr. 2417, TU Darmstadt, 2005 and arXiv:math.FA/0604128.

[71] V. S. Rabinovich, S. Roch and J. RoE: Fredholm indices of band-dominated operators, Integral Equations Operator Theory 49 (2004), no. 2, 221-238.

[72] V. S. Rabinovich, S. Roch and B. Silbermann: Fredholm Theory and Finite Section Method for Band-dominated operators, Integral Equations Operator Theory 30 (1998), no. $4,452-495$. 
[73] V. S. Rabinovich, S. Roch and B. Silbermann: Band-dominated operators with operatorvalued coefficients, their Fredholm properties and finite sections, Integral Equations Operator Theory 40 (2001), no. 3, 342-381.

[74] V. S. Rabinovich, S. Roch and B. Silbermann: Limit Operators and Their Applications in Operator Theory, Birkhäuser 2004.

[75] V. S. Rabinovich, S. Roch and B. Silbermann: The finite sections approach to the index formula for band-dominated operators, TU Darmstadt Preprint Nr. 2488, 2006.

[76] M. Reed and B. Simon: Methods of Modern Mathematical Physics III: Scattering Theory, Academic Press 1978.

[77] A. P. Robertson and W. J. Robertson: Topological Vector Spaces, Cambridge University Press 1964.

[78] S. Roch: Band-dominated operators on $\ell^{p}$-spaces: Fredholm indices and finite sections, Acta Sci. Math. 70 (2004), no. 3-4, 783-797.

[79] S. Roch: Finite Sections of Band-Dominated Operators, Memoirs of the AMS, 191, no. 895, 2008.

[80] S. Roch and B. Silbermann: Non-strongly converging approximation methods, Demonstratio Math. 22 (1989), no. 3, 651-676.

[81] R. C. Rosier: Dual spaces of certain vector sequence spaces, Pacific Journal of Mathematics 46 (1973), no. 2, 487-501.

[82] W. Rudin: Functional Analysis, 2nd Ed., McGraw-Hill 1991.

[83] B. Simon: Almost periodic Schrödinger operators: a review, Adv. Appl. Math. 3 (1982), 463-490.

[84] I. B. Simonenko: A new general method of studying linear operator equations of the type of singular integral equations, Izv. Akad. Nauk SSSR 29 (1965), 567-586 (Russian).

[85] I. B. Simonenko: On multidimensional discrete convolutions, Mat. Issled. 3 (1968), no. 1, 108-127 (Russian)

[86] M. A. Shubin: The Favard-Muhamadiev theory and pseudidifferential operators, Soviet Math. Dokl. 16 (1975), 1646-1649.

[87] M. A. Shubin: Almost periodic functions and partial differential operators, Russian Math. Surv. 33 (1978), 1-52.

[88] B. Ya. Shteinberg: Operators of the type of discrete convolution and the Noether property, Mat. Zametki 23 (1978), 417-423.

[89] G. Teschi: Jacobi Operators and Completely Integrable Nonlinear Lattices, American Mathematical Society, Providence, RI, 1999.

[90] L. N. Trefethen, M. Contedini and M. Embree: Spectra, pseudospectra, and localization for random bidiagonal matrices, Comm. Pure Appl. Math. 54 (2001), 595-623.

[91] L. N. Trefethen and M. Embree: Spectra and pseudospectra: the behavior of nonnormal matrices and operators, Princeton University Press, Princeton, NJ, 2005.

[92] V. Volpert and A. Volpert: Normal solvability and properness of elliptic operators, Partial differential equations, Amer. Math. Soc. Transl. Ser. 2 2002, 193-237.

[93] V. Volpert and A. Volpert: Properness and topological degree for general elliptic operators, Abstract Applied Analysis 2003, 129-181.

[94] A. Volpert and V. Volpert: Normal solvability of general linear elliptic problems, Abstract Applied Analysis 2005, 733-756.

[95] A. Volpert and V. Volpert: Fredholm property of general elliptic problems, Trans. Moscow Math. Soc. 67 (2006), 127-197.

[96] B. Zhang and S. N. Chandler-Wilde: Acoustic scattering by an inhomogeneous layer on a rigid plate. SIAM Journal on Applied Mathematics 58 (1998) 1931-1950.

[97] B. Zhang and S. N. Chandler-Wilde: Integral equation methods for scattering by infinite rough surfaces. Mathematical Methods in the Applied Sciences, 26 (2003), 463-488. 


\section{Index}

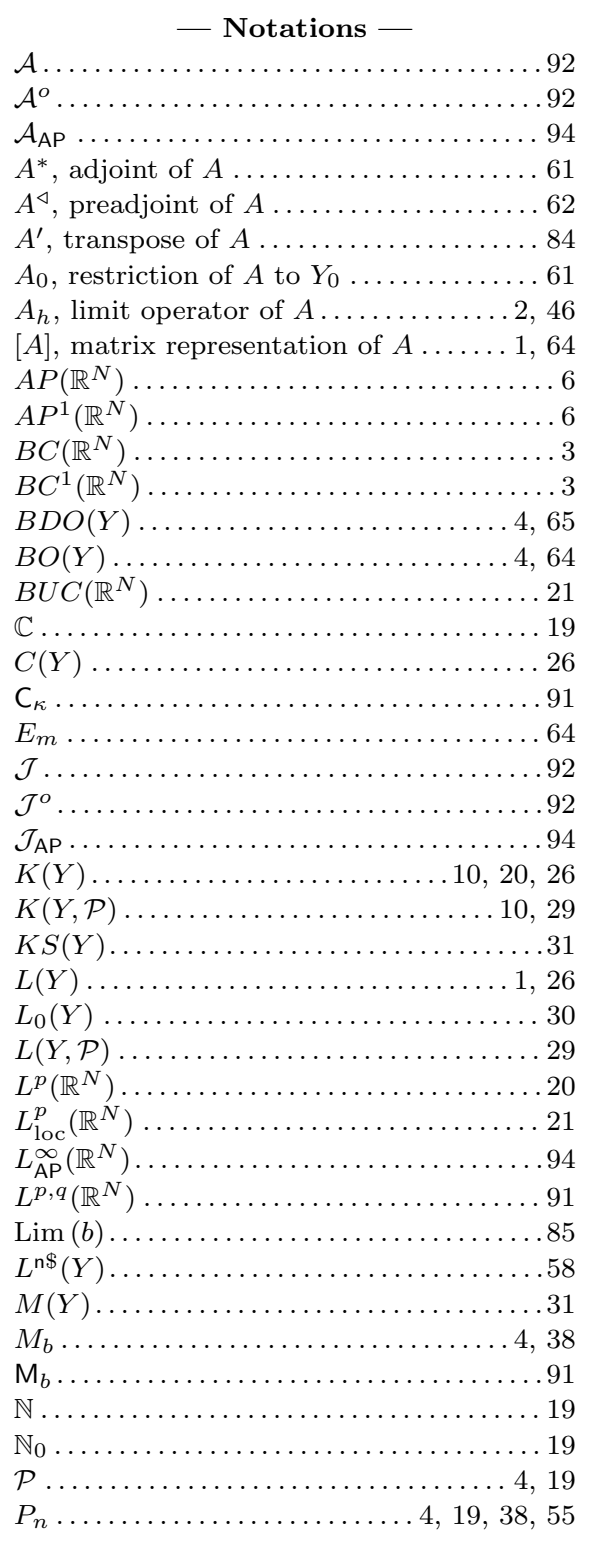

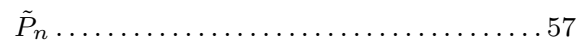

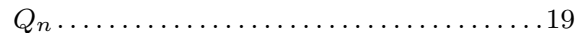

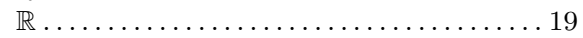

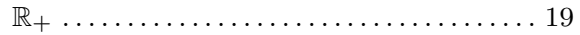

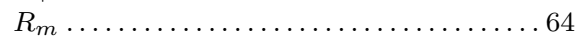

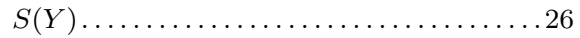

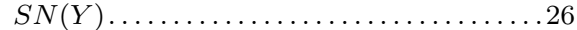

$\mathcal{T}(A) \ldots \ldots \ldots \ldots \ldots \ldots \ldots \ldots \ldots \ldots \ldots$

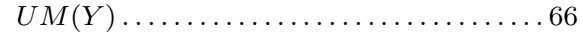

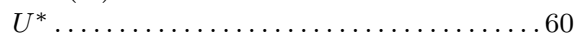

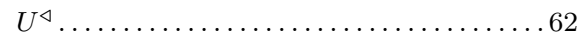

$V_{k} \ldots \ldots \ldots \ldots \ldots \ldots \ldots \ldots \ldots, 45,46,56$

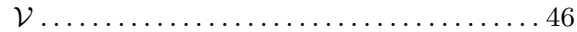

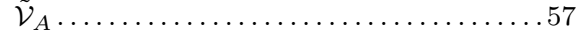

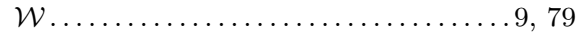

$\mathcal{W}(U) \ldots \ldots \ldots \ldots \ldots \ldots \ldots \ldots \ldots$

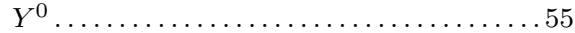

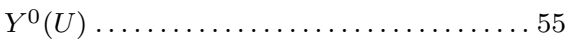

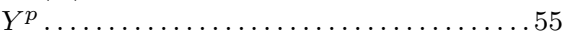

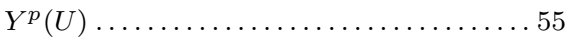

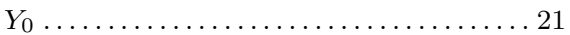

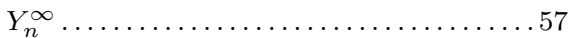

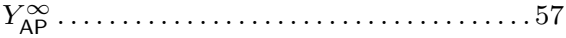

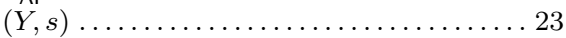

$(Y,\|\cdot\|) \ldots \ldots \ldots \ldots \ldots \ldots \ldots \ldots \ldots \ldots, 23$

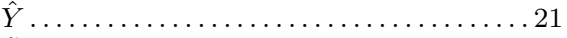

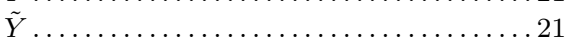

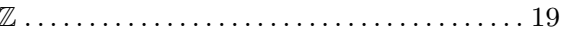

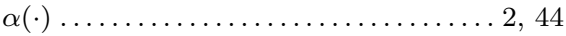

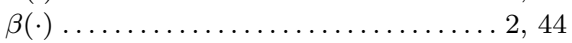

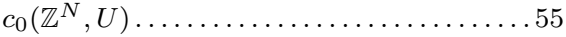

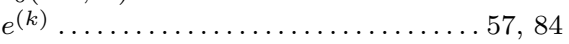

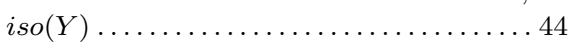

$\ell^{p}\left(\mathbb{Z}^{N}, U\right) \ldots \ldots \ldots \ldots \ldots \ldots \ldots 238$

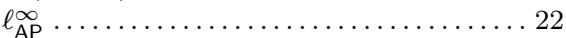

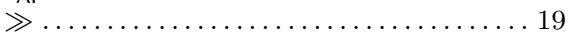

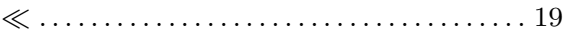

$|\cdot| \ldots \ldots \ldots \ldots \ldots \ldots \ldots \ldots \ldots \ldots \ldots \ldots . . ., 55$

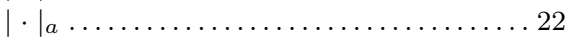

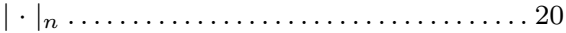

$\|\cdot\|_{\mathcal{W}} \ldots \ldots \ldots \ldots \ldots \ldots \ldots \ldots \ldots \ldots ., 79$

$\nu(A) \ldots \ldots \ldots \ldots \ldots \ldots \ldots \ldots \ldots \ldots \ldots \ldots, 13,45$

$\sigma^{\mathrm{op}}(A) \ldots \ldots \ldots \ldots \ldots \ldots \ldots \ldots \ldots \ldots \ldots, 2,47$ 
$\operatorname{spec}(A) \ldots \ldots \ldots \ldots \ldots \ldots \ldots \ldots, 69,82$

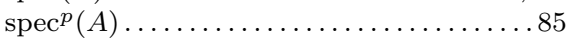

$\operatorname{spec}_{\text {ess }}(A) \ldots \ldots \ldots \ldots \ldots \ldots \ldots \ldots, 69$

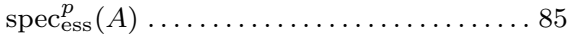

$\operatorname{spec}_{\text {point }}^{p}(A) \ldots \ldots \ldots \ldots \ldots \ldots \ldots \ldots .63 .6 \%$

$\operatorname{spec}_{\varepsilon}(A) \ldots \ldots \ldots \ldots \ldots \ldots \ldots \ldots \ldots \ldots, 69$

$\stackrel{\mathcal{P}}{\rightarrow} \ldots \ldots \ldots \ldots \ldots \ldots \ldots \ldots \ldots \ldots, 4]$

$\stackrel{s}{\rightarrow} \ldots \ldots \ldots \ldots \ldots \ldots \ldots \ldots, 4,22$

$\stackrel{S}{\rightarrow} \ldots \ldots \ldots \ldots \ldots \ldots \ldots \ldots \ldots \ldots, \ldots \ldots$

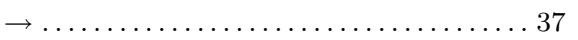

$\rightrightarrows \ldots \ldots \ldots \ldots \ldots \ldots \ldots \ldots \ldots \ldots \ldots \ldots \ldots, 37$

$-\mathbf{A}-$

absolutely rich operator .............57

adjoint operator ....................

almost Mathieu operator .............87

almost periodic function ............ 94

almost periodic matrix function .........5

almost periodic operator ............56

almost periodic sequence ..........22 57

approximate identity ................. 19

Assumption A .................... 41

asymptotically Montel ............... 51

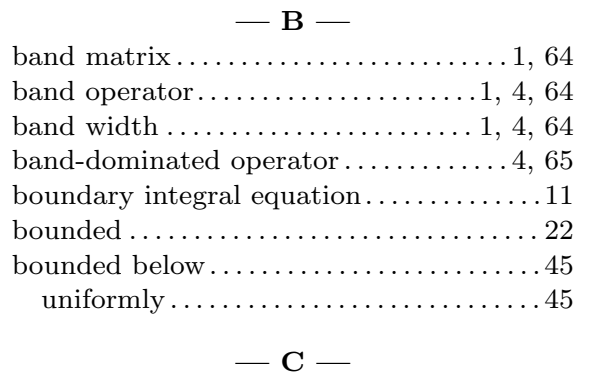

collectively compact $\ldots \ldots \ldots \ldots \ldots \ldots, 4,12$

generalised...................... 13

collectively sequentially compact ....... 32

compact ......................... 22

$s$-sequentially ................... 39

collectively .................. 4 , 12

generalised $\ldots \ldots \ldots \ldots \ldots \ldots \ldots \ldots \ldots$

relatively $\ldots \ldots \ldots \ldots \ldots \ldots \ldots \ldots, 24$

sequentially

collectively . . . . . . . . . . . . . . . . 32

relatively ....................

compact operator................ 5

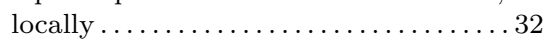

condition (i) ..................... 19

condition (ii) ......................

convergence

$\mathcal{P}-\ldots \ldots \ldots \ldots \ldots \ldots \ldots \ldots \ldots, 37$

norm .........................

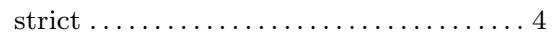

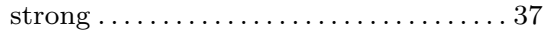

weak ..........................

weak $^{*} \ldots \ldots \ldots \ldots \ldots \ldots \ldots \ldots \ldots \ldots$

convolution operator ............ 27 91 convolution-type operator ............ 34

$$
-\mathbf{D}-
$$

differential operator $\ldots \ldots \ldots \ldots \ldots \ldots .3$

matrix ..............................

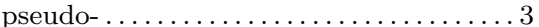

discretisation $\ldots \ldots \ldots \ldots \ldots \ldots \ldots, 2]$

dual space................... 55 60

dual system ......................60

$$
-\mathbf{E}-
$$

essential spectrum..........2, $69,82,85$

extension operator ...............6. 64

$$
-\mathbf{F}-
$$

Favard condition $\ldots \ldots \ldots \ldots \ldots \ldots, 6,78$ finite section method ................12

Fredholm index................. 2,4

Fredholm operator................ 44

semi-........................ 44

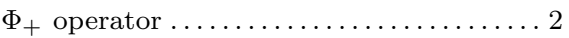

$\Phi$ - operator $\ldots \ldots \ldots \ldots \ldots \ldots \ldots \ldots \ldots, 2$

$$
\text { - } \mathbf{G}-
$$

generalised collectively compact ........13

$$
-\mathbf{H}-
$$

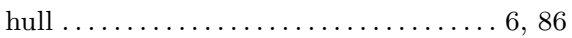

hyperplane problem .............. 81

hyperplane property $\ldots \ldots \ldots \ldots \ldots \ldots .81$

$-\mathbf{I}-$
$\operatorname{index} \ldots \ldots \ldots \ldots \ldots \ldots \ldots, 2,4 . \ldots \ldots$

integral operator $\ldots \ldots \ldots \ldots \ldots \ldots \ldots .3,34$

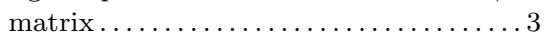

inverse closed $\ldots \ldots \ldots \ldots \ldots \ldots \ldots \ldots .29$

invertible ...................... . .

invertible at infinity ............ 10,43

$$
\begin{aligned}
& -\mathbf{J}- \\
\text { Jacobi operator } \ldots \ldots \ldots \ldots \ldots \ldots \ldots \text { 1 } & \\
& -\mathbf{L}-
\end{aligned}
$$

Laurent matrix ...................84

Laurent operator ...................84

limit function $\ldots \ldots \ldots \ldots \ldots \ldots \ldots \ldots$

limit operator .............. 4,47

weak ........................

local topology .................... 21

locally compact operator ............. 32

lower norm ................. 13,45

$$
-\mathbf{M}-
$$

matrix differential operator............ 3

matrix integral operator ..............

Montel

asymptotically .................. 51

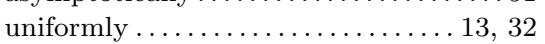

Montel operator ............... 5 (13 31

multiplication operator...........4 4 


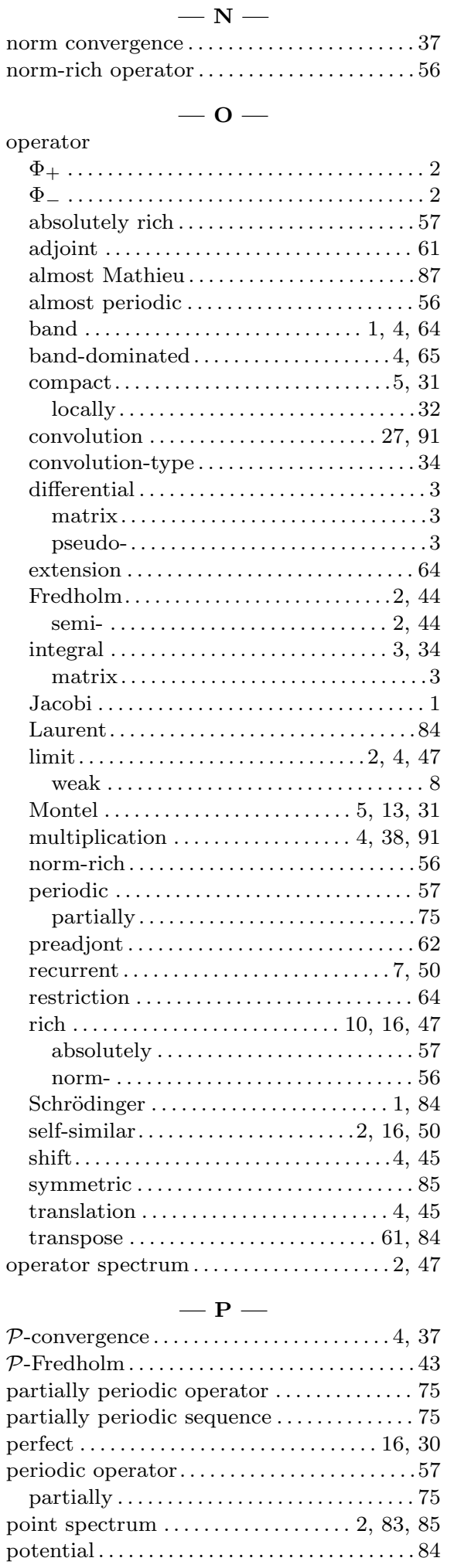

preadjoint operator $\ldots \ldots \ldots \ldots \ldots \ldots \ldots 62$ predual space ....................6 62 pseudo-differential operator ........... 3 pseudo-ergodic sequence..............88 pseudospectrum .................. 69

$$
-\mathbf{R}-
$$

random potential................... 88

recurrent operator .............. 7

relatively compact ................ 24

relatively sequentially compact ........24

restriction operator ................ 64

rich operator $\ldots \ldots \ldots \ldots \ldots \ldots \ldots, 10,16,47$

$-\mathbf{S}-$
$S$-dense $\ldots \ldots \ldots \ldots \ldots \ldots \ldots \ldots \ldots \ldots, 56 \ldots \ldots$

$s$-dense ...................... 46

$s$-sequentially compact ............ 39

Schrödinger operator .............. 1 , 84

self-similar operator ............2 16

semi-Fredholm operator ............ 24

sequentially compact

$$
\text { collectively } \ldots \ldots \ldots \ldots \ldots \ldots \ldots \ldots, 32
$$

relatively ....................... 24

shift operator ..................4, 45

slowly oscillating function ............ 94

spectrum................ 2, $69,82,85$

essential ................. $2,69,82,85$

operator .................... 2

point $\ldots \ldots \ldots \ldots \ldots \ldots \ldots \ldots \ldots, 283$

pseudo-........................69

stability ............................

strict convergence....................4

strict topology .................. 22

strong convergence................. 37

sufficient set ..................... 44

symmetric operator ............... 85

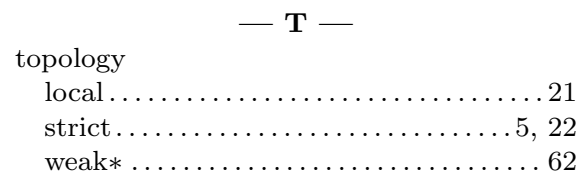

totally bounded ..................

translation operator ............. 44

transpose operator ............61 84

$$
\text { - } \mathbf{U}-
$$

uniformly bounded below ............ 45

uniformly Montel................ 13,32

unit coordinate vector ........... 57

$$
\text { - } \mathbf{W}-
$$

weak limit operator $\ldots \ldots \ldots \ldots \ldots \ldots \ldots .6$

Wiener algebra .................9, 9

$$
-\mathbf{Z}-
$$

$\mathbb{Z}$-almost periodic function ...........99 